\title{
MIXING OF COMPLEX FLUIDS WITH THE COAXIAL MIXERS COMPOSED OF TWO CENTRAL IMPELLERS AND AN ANCHOR
}

\author{
by \\ ARGANG KAZEMZADEH \\ M.Sc. Tarbiat Modarres University, Tehran, Iran, 1998 \\ B.Sc. Sharif University of Technology, Tehran, Iran, 1992
}

\author{
A Dissertation \\ Presented to Ryerson University \\ In Partial Fulfillment of the Requirements for the Degree of \\ Doctor of Philosophy \\ In the Program of Chemical Engineering
}

Toronto, Ontario, Canada, 2016

(C) Argang Kazemzadeh 2016 


\section{$\underline{\text { Author's Declaration }}$}

I hereby declare that I am the sole author of this dissertation. This is a true copy of the dissertation, including any required final revisions, as accepted by my examiners.

I authorize Ryerson University to lend this dissertation to other institutions or individuals for the purpose of scholarly research.

I further authorize Ryerson University to reproduce this dissertation by photocopying or by other means, in total or in part, at the request of other institutions or individuals for the purpose of scholarly research.

I understand that my dissertation may be made electronically available to the public. 


\section{ABSTRACT \\ Argang Kazemzadeh \\ MIXING OF COMPLEX FLUIDS WITH THE COAXIAL MIXERS COMPOSED OF TWO CENTRAL IMPELLERS AND AN ANCHOR \\ PhD, Chemical Engineering, Ryerson University, Toronto, 2016}

The coaxial mixers composed of a high-speed central impeller and a low-speed anchor have been recommended by the previous researchers for the mixing of highly viscous and nonNewtonian fluids. However, no study has been reported in the literature regarding the use of the coaxial mixing systems composed of two central impellers and an anchor in the agitation of complex fluids. Thus, the main objective of this study was to investigate the performance of coaxial mixers composed of two central impellers and an anchor in the agitation of the xanthan gum solution, which is a yield-pseudoplastic fluid, through electrical resistance tomography (ERT), the computational fluid dynamics (CFD), and design of experiments (DOE) combined with the response surface methodology (RSM).

In the first stage of this study, the hydrodynamic performance of coaxial mixers, the single and double Scaba impellers in combination with an anchor impeller, was investigated in the mixing of yield-pseudoplastic fluids. Considering the mixing efficiency criteria, it was found that the double Scaba-anchor coaxial system was more efficient than the single Scaba-anchor coaxial mixer in the mixing of yield pseudoplastic fluids with regard to the mixing time and power drawn. In the second stage of this research project, the performances of three different coaxial mixers, namely, double Scaba-anchor coaxial (DSAC), double Rushton turbine-anchor coaxial (DRAC), and double pitched blade turbine-anchor coaxial (DPAC) mixers were assessed. It was found that the double Scaba-anchor coaxial (DSAC) mixer was more efficient system compared to the others at the same operating conditions. To evaluate the influence of the impeller spacing on the hydrodynamics of the double Scaba-anchor coaxial mixer, the lower impeller clearance and the spacing between two central impellers were changed within a wide range. The results demonstrated that a coaxial mixer with the impeller spacing of almost equal to the central impeller diameter was the most efficient configuration compared to the other cases. When the impeller spacing was varied, the merging flow and parallel flow patterns were 
observed. Finally, the hydrodynamic performances of different configurations of coaxial mixers composed of a wall scraping anchor impeller in combination with two different or identical central high-speed impellers were analyzed. The coaxial mixers utilized in this stage were the Scaba-Scaba-anchor (SSAC), Scaba-Rushton-anchor (SRAC), Rushton-Scaba-anchor (RSAC), Scaba-pitched blade-anchor (SPBAC), and pitched blade-Scaba-anchor (PBSAC). A new correlation was introduced for these complex configurations of the coaxial mixers by incorporating the Metzner-Otto constants $\left(K_{s}\right)$ of the different types of the central impellers into the Reynolds number. The analysis of the collected data revealed that the Scaba-pitched bladeanchor coaxial (SPBAC) mixer was the most efficient mixing system in the mixing of the highly viscous non-Newtonian fluids. 


\section{Acknowledgements}

I would like to express the deepest appreciation to my supervisor, Professor Farhad Ein-Mozaffari, who has the attitude and substance of genius: he continually and convincingly conveyed a spirit of adventure in regard to research and scholarship, and excitement in regard to teaching. Without his guidance and persistent help this dissertation would not have been possible.

I would like to express deepest appreciation to my co-supervisors, Professor Ali Lohi and Professor Leila Pakzad, whose persistent guidance, willingness, motivation, and precious time was invaluable support for the completion of this dissertation.

I would like to thank my committee members. Professor Adel Al Taweel, Professor Simant R. Upreti, Professor Jiangning Wu, and Professor Jun Cao for their precious time and consideration.

I acknowledge all the faculty members for their great guidance, understanding, assistance, and support in regard to making this dissertation possible.

I acknowledge the assistance and great help of the administration staffs, Mrs. Alanna Mcknight, Mrs. Isabella Fernandes, and Mrs. Louise Lichacz, and also engineering staff, Mr. Ali Hemmati, Mr. Daniel Boothe, and Mr. Tondar Tajrobekar, in the Chemical Engineering Department at Ryerson University.

I am also indebted to the graduate students for their kindness, cooperation and respect given me as a graduate student to continue my research. Special thanks to Christian Bach, and Shahzad Khurram Baig.

I acknowledge Natural Sciences and Engineering Research Council of Canada (NSERC) and Ryerson Graduate Scholarship for the financial support during this work. I also acknowledge the HPCVL (High Performance Computing Virtual Laboratory) for providing the high performance computing facilities.

Special thanks to my dear friend, Branka Vuckovic, for her infinite support and unconditional love. 
This dissertation is dedicated to the following people:

- The memory of my father, K. Kazemzadeh, who has been my role-model for honesty, hard work, persistence, personal sacrifices, and his encouragement for the importance of education.

- My mother, M.K. Nigkhou, who always emphasizes the importance of education and continues to help me with my lessons throughout her life.

- My brother, Dr. A. Kazemzadeh, who has been my role-model and instilled in me the inspiration to set high goals and the confidence to achieve them.

- My brother, A. Kazemzadeh and my sisters Dr. A. Kazemzadeh. Sh. Kazemzadeh, and M. Kazemzadeh --who have been my emotional anchors through not only the vagaries of graduate school, but my entire life. 


\section{Table of Contents}

\section{Acknowledgements ..........................................................................................................v}

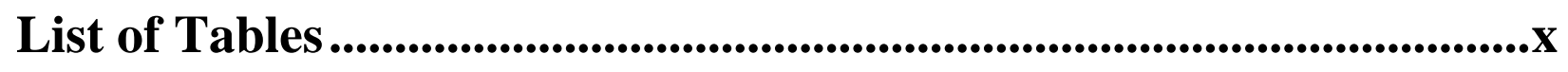

List of Figures ............................................................................................................ xii

Chapter 1 ..........................................................................................................................1

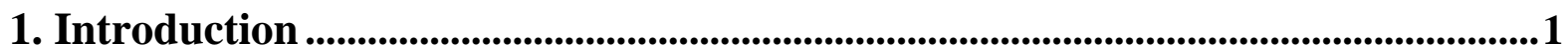

Chapter 2 .....................................................................................................................6

2. Literature Review ....................................................................................................................6

2.1 Coaxial Agitated Systems ...............................................................................................................

2.2 Power Consumption of the Coaxial Mixing Systems ............................................................8

2.3 Double Impellers Agitated Systems ............................................................................................15

2.4 Mixing Time..............................................................................................................................19

2.5 Flow Pattern ........................................................................................................................21

2.6 Research Objectives ..........................................................................................................................24

Chapter 3 .................................................................................26

3. Experimental Set-up and Procedure …......................................................................26

3.1 Experimental set-up, Material, and Methodology ...............................................................26

3.2 Electrical Resistance Tomography (ERT).............................................................................30

3.3 Fluid Rheology ....................................................................................................................31

3.4 Design of Experiments (DOE) ...............................................................................................32

3.5 Power Consumption Measurement .........................................................................................32

3.6 Mixing Time Measurement ......................................................................................................33

3.7 Error Analysis....................................................................................................................................35

3.7.1 Evaluation of Torque Sensor Precision ......................................................... 35

3.7.2 Evaluation of ERT Measurements Precision................................................... 36

\section{Chapter 4 ....................................................................................................................37}

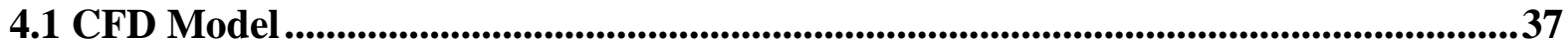

4.1 Governing Equations ............................................................................................................38

4.2 Geometry …...........................................................................................................................................38 
4.3 Grid Generation ..........................................................................................................................39

4.3.1 Grid Independency ............................................................................... 40

4.4 Modeling of Stirred Tanks, Boundary Conditions and Solver Setting............................44

4.5 Species Transport Model.................................................................................................................46

Chapter 5 .....................................................................................50

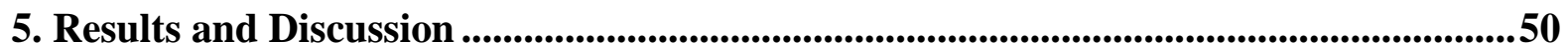

5.1 Effect of the Rheological Properties on the Mixing of Herschel-Bulkley Fluids with the Coaxial Mixers: Applications of Tomography, CFD, and Response Surface Methodology.........................................................................................................................................55

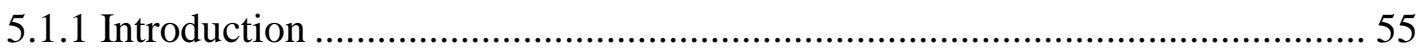

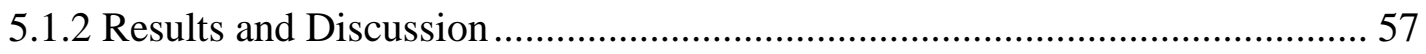

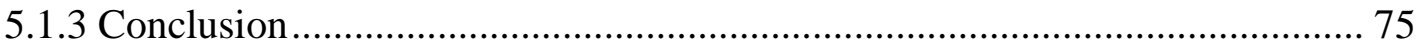

5.2 Investigation on Hydrodynamic Performances of Coaxial Mixers in Agitation of Yield-Pseudoplasitc Fluids: Single and Double Central Impellers in Combination with the Anchor ................................................................................................................................................. 77

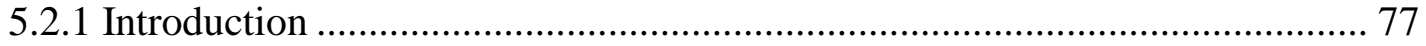

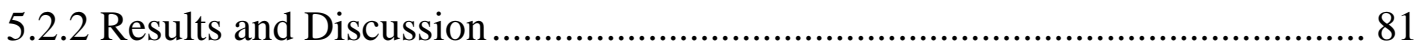

5.2.2.2 Flow Pattern ....................................................................................... 83

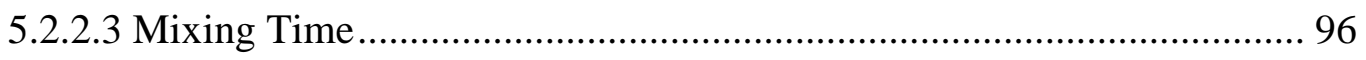

5.2.2.4 Mixing Efficiency …………………………………………………..... 97

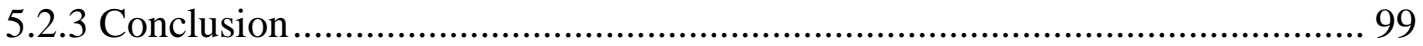

5.3 A New Perspective in the Evaluation of the Mixing of Biopolymer Solutions with Different Coaxial Mixers Comprising of Two Dispersing Impellers and a Wall Scraping Anchor.................................................................................................................................. 101

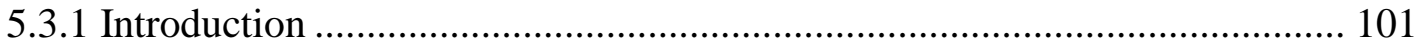

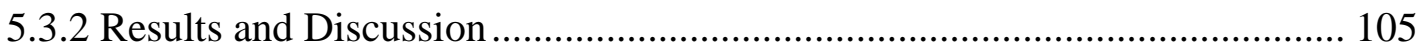

5.3.2.1 Design of Experiment........................................................................ 105

5.3.2.3 Power and Flow Numbers of the Coaxial Mixers ................................ 124

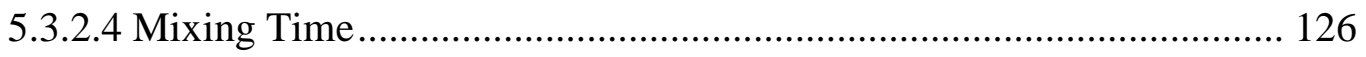

5.3.2.5 Mixing Efficiency.......................................................................... 128

5.3.4 Conclusion ................................................................................................. 129

5.4 An Investigation on the Effect of the Impeller Spacing on the Flow Field Generated by the Coaxial Mixing System Composed of Double Central Impellers and an Anchor in the Agitation of Yield-Pseudoplastic Fluids 


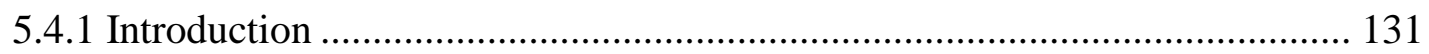

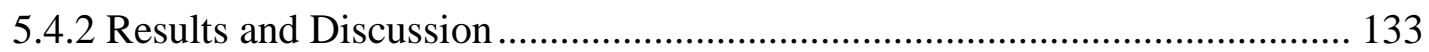

5.4.2.1 Power Number, Flow Number, Pumping Effectiveness, Mixing Time, and Mixing Efficiency .................................................................................... 133

5.4.2.2 Flow Pattern ..................................................................................... 139

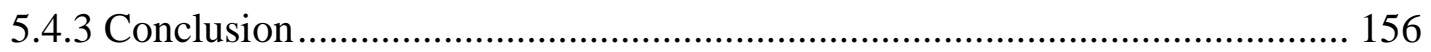

5.5 Mixing of non-Newtonian biopolymer solutions with the coaxial mixers composed of two different central impellers and an anchor ......................................................................157

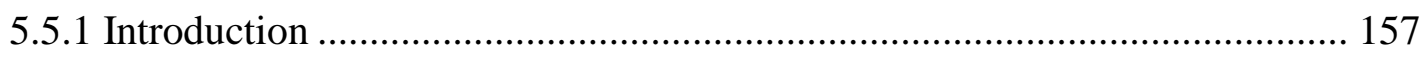

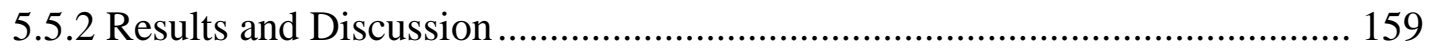

5.5.2.1 Power Consumption ....................................................................... 159

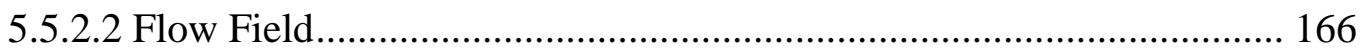

5.5.2.3 Power Number, Pumping Number, Mixing Time, and Pumping

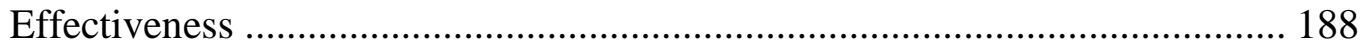

5.5.2.4 Mixing Efficiency …………………………………………………. 190

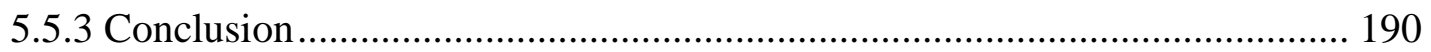

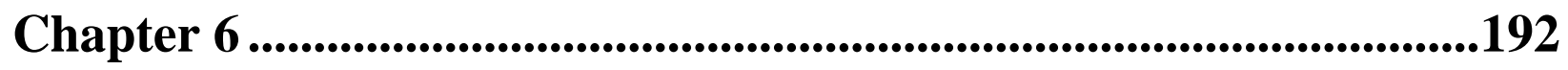

6. Overall Conclusion ....................................................................................................................192

6.1 Recommendation for Future..................................................................................................198

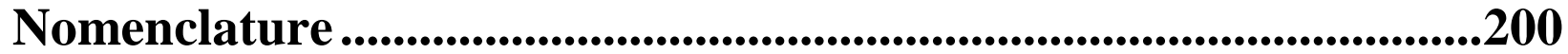

Bibliography ........................................................................................................206 


\section{List of Tables}

Table (3.3-1). Rheological properties of xanthan solutions........................ 31

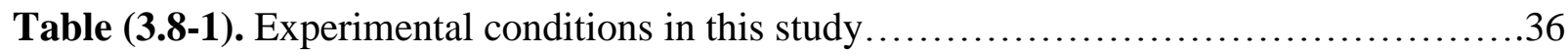

Table (4.3-1). Grid independent test results......................................... 41

Table (4.5-2) Computed and measured torque and mixing time for $0.5 \%$ xanthan gum solution agitated at $R_{n}=8$ by the Scaba-anchor coaxial mixer.

Table (5.1.1). Computed and measured torque and mixing time for $1.0 \%$ xanthan gum solution agitated by the Scaba-anchor coaxial mixer.................................. 56

Table (5.1-2). Experimental range and levels of the independent variables.................57

Table (5.1-3). ANOVA test for response function (mixing time) $t_{m}=180+52.21 \mathrm{~K}+42.21 \tau_{y}-$

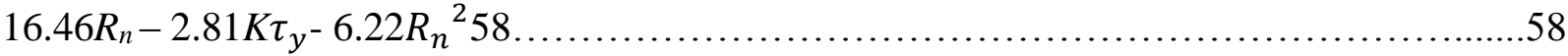

Table (5.1-4). ANOVA test for response function (power consumption) $P=23.42-1.85 n+$ $9.44 K+2.34 \tau_{y}+15.05 R_{n}-1.19 n K-1.45 n R_{n}+1.26 K \tau_{y}+4.72 K R_{n}-1.22 \tau_{y}{ }^{2}+3.06 R_{n}{ }^{2}$

Table (5.1-5). Coefficients of $N . t_{m}$ vs. $a R e^{b}$ as a function of the speed ratio when the consistency index $(\mathrm{K})$ changes from 3 to $32 \mathrm{~Pa}_{\mathrm{s}} \mathrm{s}^{\mathrm{n}}$

Table (5.1-6). Coefficients of $N . t_{m}$ vs. $a \operatorname{Re}^{b}$ as a function of the speed ratio when the power-

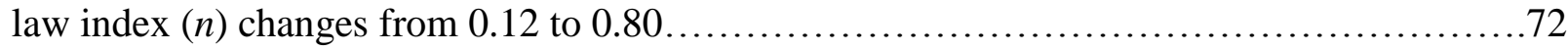

Table (5.1-7). Coefficients of $N . t_{m}$ vs. $a \operatorname{Re}^{b}$ as a function of the speed ratio when the yield-

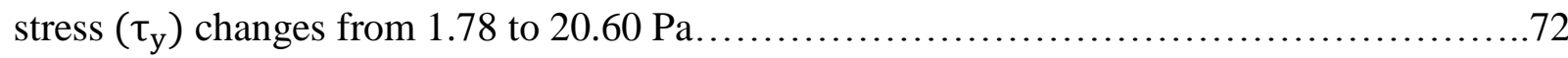

Table (5.1-8). Coefficients of $t_{m}$ vs. $P / V$ as a function of the consistency index $(K) \ldots \ldots \ldots 74$

Table (5.1-9). Coefficients of $t_{m}$ vs. $P / V$ as a function of the power-law index $(n) \ldots \ldots \ldots \ldots 75$

Table (5.1-10). Coefficients of $t_{m}$ vs. $P / V$ as a function of the yield stress $\tau_{y} \ldots \ldots \ldots \ldots \ldots 75$

Table (5.2-1). The experimental conditions..........................................

Table (5.2-2). Computed and measured torque and mixing time for $0.5 \%$ xanthan gum solution agitated at $R_{n}=8$ by the Scaba-anchor coaxial mixer.......................... 79

Table (5.3-1). Operating conditions employed in this study.............................. 102 
Table (5.3-2). Experimental range and levels of the independent variables .103

Table (5.3-3). Computed and measured torque and mixing time for $1 \%$ xanthan gum solution agitated at $R_{n}=8$ by Scaba-anchor coaxial mixer. 105

Table (5.3-4). ANOVA test for response function (mixing time) $Y_{1}=11.90-1.82 X_{1}+3.70 X_{2}-$ $2.03 X_{3}+1.59 X_{1} X_{2}+5.06 X_{1}^{2}-1.45 X_{2}^{2}-3.79 X_{3}^{2}\left(R^{2}=0.9840\right)$. 106

Table (5.3-5). ANOVA test for response function (power consumption) $Y_{2}=659.87+148.16 X_{1}$ $+48.46 X_{2}+362.36 X_{3}+111.06 X_{1} X_{3}-142.76 X_{2} X_{3}-214.95 X_{1}^{2}+139.31 X_{3}^{2}\left(R^{2}=0.9719\right) \ldots 106$

Table (5.3-6). The power number and flow number of the three coaxial mixers.... 126

Table (5.4-1). Configurations of the coaxial mixer.

Table (5.4-2). Computed and measured power consumption and mixing time for different configurations of coaxial mixer at $R_{n}=8\left(N_{a}=30 \mathrm{rpm}\right.$ and $\left.N_{s}=240 \mathrm{rpm}\right)$ and $1.5 \%$ xanthan gum solution in the co-rotating mode.

Table (5.4-3). Power number, flow number, pumping effectiveness and mixing time for different configurations of coaxial mixer at $R_{n}=8\left(\mathrm{Na}=30 \mathrm{rpm}\right.$ and $\left.N_{s}=240 \mathrm{rpm}\right)$ and $1.5 \%$ xanthan gum solution in the co-rotating mode 136

Table (5.4-4). Power number, flow number, pumping effectiveness and mixing time for different configuration of coaxial mixer at $R_{n}=8\left(N_{a}=30 \mathrm{rpm}\right.$ and $\left.N_{s}=240 \mathrm{rpm}\right)$ and $1.0 \%$ xanthan gum solution in the counter-rotating mode 137

Table (5.5-1). Computed and measured power consumption and mixing time for five coaxial mixers at $R_{n}=8\left(N_{a}=30 \mathrm{rpm}\right.$ and $\left.N_{s}=240 \mathrm{rpm}\right), 1.5 \%$ xanthan gum solution, and co-rotating mode.

Table (5.5-2). Power number, flow number, pumping effectiveness, and mixing time for five coaxial mixers at $R_{n}=8\left(N_{a}=30 \mathrm{rpm}\right.$ and $\left.N_{s}=240 \mathrm{rpm}\right), 1.5 \%$ xanthan gum solution, and corotating mode. 189 


\section{List of Figures}

Figure (2.5-1). Flow patterns generated by: (a) a radial-flow impeller, and (b) an axial-flow

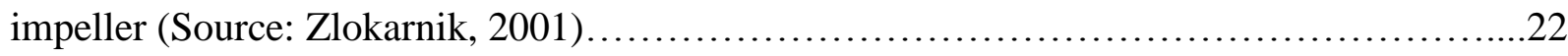

Figure (2.5-2). Flow pattern generated by an anchor impeller (Source: Taterson, 1991)........22

Figure (2.5-3). Stable flow patterns generated in a dual-Rushton turbine stirred vessel (a) Parallel flow; (b) merging flow; (c) diverging flow (Source: Rutherford, 1997)..............23

Figure (3.1-1). Schematic diagram of experimental setup.............................28

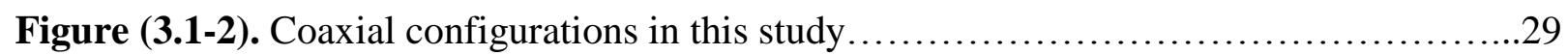

Figure (3.6-1). ERT planes and Mean conductivity measured using ERT as a function of time for $2.0 \%$ xanthan gum solution agitated by double Scaba-Anchor coaxial (DSAC) at $N_{a}=30$

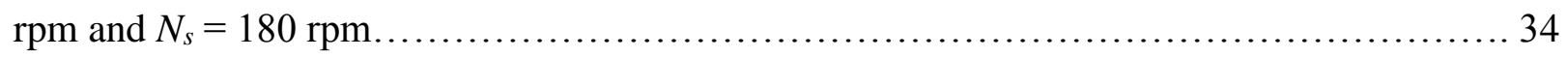

Figure (3.6-2). $2 \mathrm{D}$ and 3D tomography images at $R_{n}=8$, and $1 \%$ xanthan gum solution in the co-rotating mode for: (a) injection point at $t=100 \mathrm{~s}$, and (b) at $t=150 \mathrm{~s}$ by double Scaba-

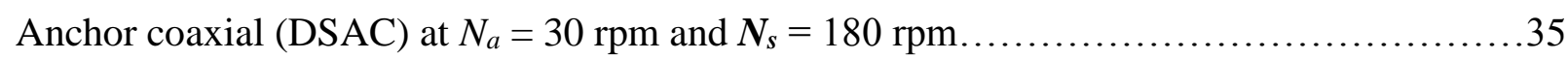

Figure (4.2-1). Geometry of developed model with specified zones..........................39

Figure (4.3-1). Unstructured tetrahedral mesh for coaxial system.........................40

Figure (4.3-2). Selected lines for grid independence test ................................42

Figure (4.3-3). Effect of the number of grid on tangential velocity in horizontal position close

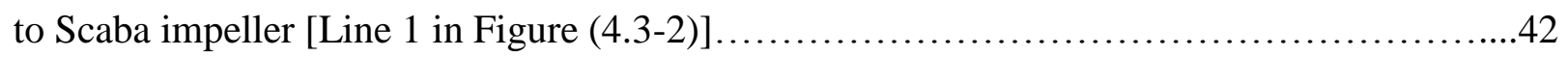

Figure (4.3-4). Effect of the number of grids on (a) tangential velocity, and (b) Radial velocity in horizontal position close to anchor impeller [Line 2 in Figure (4.3-2)] ..........................43

Figure (4.3-5). Effect of the number of grids on axial velocity in vertical situation close to anchor impeller [Line 3 in Figure (4.3-2)]. 
Figure (4.5-1). Monitoring planes and normalized tracer concentration using simulated CFD model as a function of time for $1.0 \%$ xanthan gum solution agitated by Scaba-Scaba-Anchor coaxial $(\mathrm{SSAC})$ at $N_{a}=30 \mathrm{rpm}$ and $N_{s}=180 \mathrm{rpm}$.

Figure (5.1-1). The configuration of the coaxial mixers used in this study .55

Figure (5.1-2). Predicted values versus the experimental data for (a) mixing time (s) and (b) power consumption $(\mathrm{W})$....

Figure (5.1-3) Response surface methodology (RSM) showing mixing time as a function of two independent variables (other variables were held at their respective center levels): (a) power-law index $(n)$ and consistency index $(K),(\mathbf{b})$ power-law index $(n)$ and yield stress $\left(\tau_{y}\right)$, (c) power-law index $(n)$ and speed ratio $\left(R_{n}\right)$, (d) consistency index $(K)$ and yield stress $\left(\tau_{y}\right),(\mathbf{e})$ consistency index $(K)$ and speed ratio $\left(R_{n}\right)$, and (f) yield stress $\left(\tau_{y}\right)$ and speed ratio $\left(R_{n}\right)$

Figure (5.1-4). Response surface methodology (RSM) showing power consumption as a function of two independent variables (other variables were held at their respective center levels): (a) power-law index $(n)$ and consistency index $(K)$, (b)power-law index $(n)$ and yield stress $\left(\tau_{y}\right)$, (c) power-law index $(n)$ and speed ratio $\left(R_{n}\right),(\mathbf{d})$ consistency index $(K)$ and yield stress $\left(\tau_{y}\right)$, (e) consistency index $(K)$ and speed ratio $\left(R_{n}\right)$, and (f) yield stress $\left(\tau_{y}\right)$ and speed ratio $\left(R_{n}\right)$.

Figure (5.1-5). Viscosity (kg/m-s) contour plots depicting the effect power-law index on the mixing system produced by the central impeller using vertical cross section planes for $1 \%$ xanthan gum concentration agitated at: (a) $n=0.12$ (b) $n=0.8$ (c) at $K=8, \tau_{y}=5.254$, and $R n=$ 5.

Figure (5.1-6). Velocity $(\mathrm{m} / \mathrm{s})$ vector plots for effect of consistency index on the mixing system: (a) $K=8 \mathrm{Pas}^{\mathrm{n}}$ and (b) $K=28 \mathrm{Pas}^{\mathrm{n}}$ for Scaba-anchor impeller agitated at $N_{a}=30 \mathrm{rpm}$, $N_{s}=150 \mathrm{rpm}, n=0.12, \tau_{y}=5.254 \mathrm{~Pa}$

Figure (5.1-7). Velocity $(\mathrm{m} / \mathrm{s})$ vector plots in vertical direction as a function of the speed ratio for the Scaba-anchor impeller at $1.0 \%$ xanthan gum solution, $n=0.12, K=8 \mathrm{Pas}^{\mathrm{n}}$, and $\tau_{y}=$ $5.254 \mathrm{~Pa}:$ (a) $N_{s}=50 \mathrm{rpm}, N_{a}=30 \mathrm{rpm}, R_{n}=1.7$ and (b) $N_{s}=180 \mathrm{rpm}, N_{a}=30 \mathrm{rpm}, R_{n}=$ 6 .67

Figure (5.1-8). Three dimensional streamlines (3D) plots produced by the central impeller using vertical cross section planes for $1 \%$ xanthan gum concentration agitated for: (a) $\tau_{y}=$

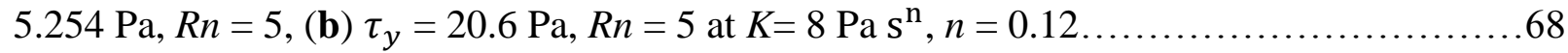

Figure (5.1-9). Correlation between dimensionless mixing time and generalized Reynolds number for the effect of: (a) consistency index $(K)$, (b) power-law-index $(n)$, and (c) yield stress $\left(\tau_{y}\right)$ at five speed ratio for $1.0 \%$ xanthan gum solution 
Figure (5.1-10). Correlation between mixing time and specific power consumption for the effect of: (a) consistency index $(K)$, (b) power-law-index $(n)$, and (c) yield stress $\left(\tau_{y}\right)$ at five speed ratios for $1.0 \%$ xanthan gum solution..................................... 74

Figure (5.2-1). Experimental set-up and configurations of the coaxial mixers used in this study.... 78

Figure (5.2-2). Normalized tracer concentration versus time at Point A $(x=0.13, y=0.03$, and $Z=0.360 \mathrm{~m})$ and Point B $(x=0.00, y=0.00$, and $Z=0.140 \mathrm{~m})$ for the DSAC mixer at $R_{n}=8$ $\left(N_{s}=112 \mathrm{rpm}, N_{\mathrm{a}}=14 \mathrm{rpm}\right)$ and $0.5 \%$ xanthan gum solution: (a) CFD results and (b) ERT results. .80

Figure (5.2-3). Power curves for the double Scaba-anchor coaxial mixer at different speed ratios in the co-rotating mode and 1\% xanthan gum solution obtained using (a) Pakzad et al. model and (b) Bao et al. model........................................................ 82

Figure (5.2-4). Power curves for the double Scaba-anchor coaxial mixer at different xanthan gum solutions in the co-rotating mode at the speed ratio $R_{n}=8$ in co-rotating mode for: (a) Pakzad model and (b) Bao model.......................................................83

Figure (5.2-5). 2D and 3D tomography images at $P=190.5 \mathrm{~W} / \mathrm{m}^{3}, R_{n}=8$, and $1 \%$ xanthan gum solution in the co-rotating mode for: (a) SSAC mixer at $t=20 \mathrm{~s}$, (b) DSAC mixer at $t=20$

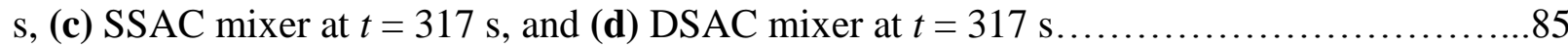

Figure (5.2-6). Vertical and horizontal velocity $(\mathrm{m} / \mathrm{s})$ contour plots at $P=190.5 \mathrm{~W} / \mathrm{m}, R_{n}=8$, and $1 \%$ xanthan gum solution in the co-rotating mode for: (a) Single Scaba-anchor coaxial (SSAC) mixer and (b) Double Scaba-anchor coaxial (DSAC) mixer... .87

Figure (5.2-7). Vertical and horizontal velocity $(\mathrm{m} / \mathrm{s})$ vector plots at $P=190.5 \mathrm{~W} / \mathrm{m}^{3}, R_{n}=8$, and $1 \%$ xanthan gum solution in the co-rotating mode for: (a) Single Scaba-anchor coaxial (SSAC) mixer and (b) Double Scaba-anchor coaxial (DSAC) mixer. .89

Figure (5.2-8). Dimensionless axial velocity profiles along the tank height for both single Scaba-anchor coaxial (SSAC) with Scaba $\left(Z_{1} / H\right)=0.34$ and double Scaba-anchor coaxial (DSAC) mixers with two Scaba $\left(\mathrm{Z}_{1} / \mathrm{H}=0.34\right.$ and $\left.\mathrm{Z}_{2} / \mathrm{H}=0.72\right)$ at $2 r / D=0.45$, from $y=-0.09$ to $y=+0.09, P=190.5 \mathrm{~W} / \mathrm{m}^{3}, R_{n}=8$, and $1 \%$ xanthan gum solution in the co-rotating mode for: (a) axial velocity, (b) radial velocity, and (c) tangential velocity..... .92

Figure (5.2-9). Dimensionless average shear strain rate along the tank height for both single Scaba-anchor coaxial $(S S A C)$ with Scaba $\left(Z_{1} / H=0.34\right)$ and double Scaba-anchor coaxial $(D S A C)$ mixers with two scaba $\left(Z_{1} / H=0.34\right.$ and $\left.Z_{2} / H=0.72\right)$ at different radial positions $(2 r / D$ $=0.25,0.45,0.55$, and 0.65$), P=190.5 \mathrm{~W} / \mathrm{m}^{3}, R_{n}=8$, and $1 \%$ xanthan gum solution in the corotating mode for: (a) single Scaba-anchor coaxial (SSAC) mixer and (b) double Scaba-anchor coaxial (DSAC) mixer. .94

Figure (5.2-10). Vertical and horizontal viscosity (kg/m s) contour plots at $P=190.5 \mathrm{~W} / \mathrm{m}^{3}, R_{n}$ $=8$, and $1 \%$ xanthan gum solution in the co-rotating mode for: (a) single Scaba-anchor coaxial (SSAC) mixer and (b) double Scaba-anchor coaxial (DSAC) mixer... . .95 
Figure (5.2-11). Mixing time versus total specific power consumption for $1 \%$ xanthan gum solution for both single Scaba-anchor coaxial (SSAC) and double Scaba-anchor coaxial (DSAC) mixers in the co-rotating mode: (a) $R_{n}=3$, (b) $R_{n}=6$, and (c) $R_{n}=8$.

Figure (5.2-12) Mixing energy versus $R e_{m}$ number at $R_{n}=8$ and $1 \%$ xanthan gum solution in the co-rotating mode for both single Scaba-anchor coaxial (SSAC) and double Scaba-anchor coaxial (DSAC) mixers.

Figure (5.3-1). Schematic diagram of the experimental set-up and different coaxial mixer configurations analyzed in this study.... 102

Figure (5.3-2). Predicted values versus the experimental data for (a) mixing time $(s)$ and (b) specific power consumption $\left(\mathrm{W} / \mathrm{m}^{3}\right)$.

Figure (5.3-3). Response surface methodology (RSM) results showing mixing time and specific power drawn as a function of two independent variables (other variables were fixed): (a) coaxial mixer $\left(X_{1}\right)$ and concentration $\left(X_{2}\right)$, (b) coaxial mixer $\left(X_{1}\right)$ and central impeller speed

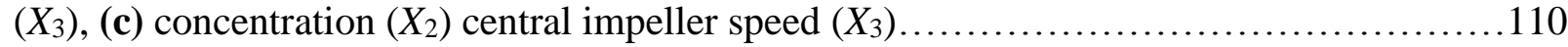

Figure (5.3-4). Vertical and horizontal velocity $(\mathrm{m} / \mathrm{s})$ vector plots at $R_{n}=8\left(N_{c}=240 \mathrm{rpm}\right.$ and $N_{a}=30 \mathrm{rpm}$ ), and $1.5 \%$ xanthan gum solution for: (a) double Scaba-anchor coaxial mixer (DSAC) mixer, (b) double Rushton-anchor coaxial (DRAC) mixer, and (c) double pitched blade-anchor coaxial (DPAC) mixer. 113

Figure (5.3-5). Streamline plots at $R_{n}=8\left(N_{c}=240 \mathrm{rpm}\right.$ and $\left.N_{a}=30 \mathrm{rpm}\right)$, and $1.5 \%$ xanthan gum solution for: (a) double Scaba-anchor coaxial mixer (DSAC) mixer, (b) double Rushtonanchor coaxial (DRAC) mixer, and (c) Double pitched blade-anchor coaxial (DPAC) mixer. .114

Figure (5.3-6). Normalized velocity profiles along the tank wall at $2 r / T=0.45, R_{n}=8\left(N_{c}=\right.$ $240 \mathrm{rpm}$ and $N_{a}=30 \mathrm{rpm}$ ), and 1.5\% xanthan gum solution: (a) axial velocity (b) radial

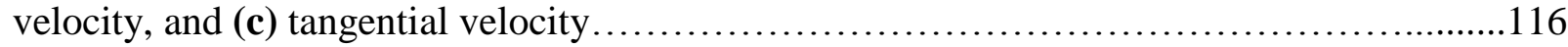

Figure (5.3-7). Radial profiles of axial, radial, and tangential velocities at $R_{n}=8\left(N_{c}=240\right.$ rpm and $N_{a}=30 \mathrm{rpm}$ ), and 1.5\% xanthan gum solution : (a) axial velocity under the lower impeller along the line parallel to the $x$-axis with $Z / H=0.24$, (b) axial velocity above the lower impeller along the line parallel to the $x$-axis with $Z / H=0.36$, (c) radial velocity under the lower impeller along the line parallel to the $x$-axis with $Z / H=0.24$, , (d) radial velocity above the lower impeller along the line parallel to the $x$-axis with $Z / H=0.36$, (e) tangential velocity under the lower impeller along the line parallel to the $x$-axis with $Z / H=0.24$, and (f) tangential velocity above the lower impeller along the line parallel to the $x$-axis with $Z / H=$ 0.36 118

Figure (5.3-8). Velocity (m/s) contour plots at $R_{n}=8\left(N_{c}=240 \mathrm{rpm}\right.$ and $\left.N_{a}=30 \mathrm{rpm}\right)$, and $1.5 \%$ xanthan gum solution for: (a) axial velocity, (b) radial velocity, and (c) tangential velocity.... 121

Figure (5.3-9). Dimensionless average shear rate plots at $R_{n}=8\left(N_{c}=240 \mathrm{rpm}\right.$ and $N_{a}=30$ $\mathrm{rpm}$ ) and $1.5 \%$ xanthan gum solution along a line parallel to the $z$-axis (a) $2 r / T=0.25$, (b) $2 r / T=$ 0.45, (c) $2 r / T=0.55$, and (d) $2 r / T=0.65$. 
Figure (5.3-10). Vertical and horizontal viscosity $(\mathrm{kg} / \mathrm{m} . \mathrm{s})$ contour plots at $R_{n}=8\left(N_{c}=240\right.$ rpm and $N_{a}=30 \mathrm{rpm}$ ) and 1.5\% xanthan gum solution: (a) double Scaba-anchor coaxial (DSAC) mixer, (b) double Rushton-anchor coaxial (DRAC) mixer, and (c) double pitched blade turbine (DPAC) mixer. 124

Figure (5.3-11). Normalized tracer concentration verses time at $R_{n}=8\left(N_{c}=240 \mathrm{rpm}\right.$ and $N_{a}=$ $30 \mathrm{rpm})$ and $1.5 \%$ xanthan gum solution: (a) between two central impellers at position $(0.13$, $0.03,0.25 \mathrm{~m})$ and $(\mathbf{b})$ under the lower impeller at position $(0.00,0.00,0.12 \mathrm{~m})$. 127

Figure (5.3-12). $\left(1 / \pi_{1}\right)$ verses speed ratio for the double Scaba-anchor coaxial (DSAC), double Rushton-anchor coaxial (DRAC), and double pitched blade turbine-anchor (DPAC) mixers at $1.5 \%$ xanthan gum solution. 128

Figure (5.4-1). Experimental Set-up in this study 131

Figure (5.4-2). Power number and mixing time versus the impeller spacing $(\mathrm{m})$ for different coaxial mixing configurations at $R_{n}=8\left(N_{a}=30 \mathrm{rpm}\right.$ and $\left.N_{s}=240 \mathrm{rpm}\right)$ and $1.5 \%$ xanthan gum solution in co-rotating mode.... 138

Figure (5.4-3). Energy consumption as a function of the impeller spacing for different coaxial mixing configurations at $R_{n}=8\left(N_{a}=30 \mathrm{rpm}\right.$ and $\left.N_{s}=240 \mathrm{rpm}\right)$ and $1.5 \%$ xanthan gum solution in the co-rotating mode.

Figure (5.4-4). Velocity $(\mathrm{m} / \mathrm{s})$ contour plots generated by different coaxial mixing configurations at $R_{n}=8\left(N_{a}=30 \mathrm{rpm}\right.$ and $\left.N_{s}=240 \mathrm{rpm}\right)$ and $1.5 \%$ xanthan gum solution in the co-rotating mode: (a) SS1, (b) SS2, (c) SS3, (d) SS4, (e) SS5, and (f) SS6................141

Figure (5.4-5) Velocity $(\mathrm{m} / \mathrm{s})$ vector plots generated by different coaxial mixing configurations at $R_{n}=8\left(N_{a}=30 \mathrm{rpm}\right.$ and $\left.N_{s}=240 \mathrm{rpm}\right)$ and $1.5 \%$ xanthan gum solution in the co-rotating mode: (a) SS1, (b) SS2, (c) SS3, (d) SS4, (e) SS5, and (f) SS6. 144

Figure (5.4-6). Streamlines $(\mathrm{m} / \mathrm{s})$ plots generated by different coaxial mixing configurations at $R_{n}=8\left(N_{a}=30 \mathrm{rpm}\right.$ and $\left.N_{s}=240 \mathrm{rpm}\right)$ and $1.5 \%$ xanthan gum solution in the co-rotating mode: (a) SS1, (b) SS2, (c) SS3, (d) SS4, (e) SS5, and (f) SS6. 145

Figure (5.4-7). Axial profiles of the radial, axial, and tangential velocities at $2 r / T=0.55$ for different coaxial mixing configurations at $R_{n}=8\left(N_{a}=30 \mathrm{rpm}\right.$ and $\left.N_{s}=240 \mathrm{rpm}\right)$ and $1.5 \%$ xanthan gum solution in the co-rotating mode 148

Figure (5.4-8). Radial profiles of the radial velocity at the impeller tip $\left(R_{\mathrm{i}}\right)$ for different coaxial mixing configurations at $R_{n}=8\left(N_{a}=30 \mathrm{rpm}\right.$ and $\left.N_{s}=240 \mathrm{rpm}\right)$ and $1.5 \%$ xanthan gum solution in the co-rotating mode: (a) lower impeller, and (b) upper impeller. 149

Figure (5.4-9). Radial profiles of the axial velocity at $R_{n}=8\left(N_{a}=30 \mathrm{rpm}\right.$ and $\left.N_{s}=240 \mathrm{rpm}\right)$ and $1.5 \%$ xanthan gum solution in the co-rotating mode for: (a) $15 \mathrm{~mm}$ above the upper impeller, (b) $15 \mathrm{~mm}$ below the upper impeller, (c) $15 \mathrm{~mm}$ above the lower impeller, and (d) 15 $\mathrm{mm}$ below the lower impeller. 151

Figure (5.4-10). Radial profile of the radial velocity at $R_{n}=8\left(N_{a}=30 \mathrm{rpm}\right.$ and $\left.N_{s}=240 \mathrm{rpm}\right)$ and $1.5 \%$ xanthan gum solution in the co-rotating mode for: (a) $15 \mathrm{~mm}$ above the upper 
impeller, (b) $15 \mathrm{~mm}$ below the upper impeller, (c) $15 \mathrm{~mm}$ above the lower impeller, and (d) 15 $\mathrm{mm}$ below the lower impeller.

Figure (5.4-11). Dimensionless average shear strain rate plots along the tank wall generated by different coaxial mixing configurations at $R_{n}=8\left(N_{a}=30 \mathrm{rpm}\right.$ and $\left.N_{s}=240 \mathrm{rpm}\right)$ and $1.5 \%$ xanthan gum solution in the co-rotating mode: (a) SS1, (b) SS2, (c) SS3, (d) SS4, (e) SS5, and (f) SS6.

Figure (5.4-12). Viscosity $(\mathrm{kg} / \mathrm{m}-\mathrm{s})$ contour plots generated by different coaxial mixing configurations at $R_{n}=8\left(N_{a}=30 \mathrm{rpm}\right.$ and $\left.N_{s}=240 \mathrm{rpm}\right)$ and $1.5 \%$ xanthan gum solution in the co-rotating mode: (a) SS1, (b) SS2, (c) SS3, (d) SS4, (e) SS5, and (f) SS6.................155

Figure (5.5-1). Different configurations of coaxial mixers: Scaba-Scaba-anchor coaxial (SSAC), (b) Rushton-Scaba-anchor coaxial (RTSAC), Scaba-Rushton-anchor coaxial (SRAC), Scaba-pitched blade-anchor coaxial (SPAC), and pitched blade-Scaba-anchor coaxial (PSAC) mixers in this study. .158

Figure (5.5-2). Average shear rate (1/s) versus the impeller speed (1/s) at $R_{n}=8$ and corotating mode as a function of the xanthan gum concentration for: (a) single Scaba, (b) single Rushton turbine, and (c) single pitched blade impellers. 162

Figure (5.5-3). Average shear rate (1/s) versus the impeller speed (1/s) at $R_{n}=8$ and corotating mode as a function of the xanthan gum concentration for: (a) Scaba-pitched blade coaxial mixer and (b) Scaba-Rushton-anchor coaxial mixer........................... 163

Figure (5.5-4). The master power curves for five coaxial mixers at $R_{n}=8,1.5 \%$ xanthan gum solution, and the co-rotating mode.... 165

Figure (5.5-5). Velocity (m/s) contour plots at $R_{n}=8\left(N_{c}=240 \mathrm{rpm}\right.$ and $\left.N_{a}=30 \mathrm{rpm}\right)$ and $1.5 \%$ xanthan gum solution: (a) axial velocity, (b) radial velocity, and (c) tangential velocity. 168

Figure (5.5-6). Normalized axial profiles of the axial velocity along the tank wall at $R_{n}=8\left(N_{c}\right.$ $=240 \mathrm{rpm}$ and $N_{a}=30 \mathrm{rpm}$ ) and $1.5 \%$ xanthan gum solution: (a) $2 r / T=0$, (b) $2 r / T=0.45$, (c) $2 \mathrm{r} / \mathrm{T}=0.58$, and (d) $2 r / T=0.68$.

Figure (5.5-7). Normalized axial profiles of the radial velocity along the tank wall at $R_{n}=8\left(N_{c}\right.$ $=240 \mathrm{rpm}$ and $N_{a}=30 \mathrm{rpm}$ ) and $1.5 \%$ xanthan gum solution: (a) $2 r / T=0.25$, (b) $2 r / T=0.45$, (c) $2 r / T=0.58$, and (d) $2 r / T=0.68$. 175

Figure (5.5-8) Normalized axial profiles of the tangential velocity along the tank wall at $R_{n}=8$ $\left(N_{c}=240 \mathrm{rpm}\right.$ and $\left.N_{a}=30 \mathrm{rpm}\right)$ and $1.5 \%$ xanthan gum solution: (a) $2 r / T=0.25$, (b) $2 r / T=$ 0.45, (c) $2 r / T=0.58$, and (d) $2 r / T=0.68$ .178

Figure (5.9-9). Normalized radial profiles of the axial velocity along the tank wall at $R_{n}=8\left(N_{c}\right.$ $=240 \mathrm{rpm}$ and $N_{a}=30 \mathrm{rpm}$ ) and $1.5 \%$ xanthan gum solution: (a) $z / H=0.20$, (b) $z / H=0.50$, and (c) $z / H=0.86$. 180

Figure (5.5-10). Normalized radial profiles of the radial velocity along the tank wall at $R_{n}=8$ ( $N_{c}=240 \mathrm{rpm}$ and $N_{a}=30 \mathrm{rpm}$ ) and $1.5 \%$ xanthan gum solution: (a) $z / H=0.20$, (b) $z / H=$ 0.50 , and (c) $z / H=0.86$. 182 
Figure (5.5-11). Normalized radial profiles of the tangential velocity along the tank wall at $R_{n}$ $=8\left(N_{c}=240 \mathrm{rpm}\right.$ and $\left.N_{a}=30 \mathrm{rpm}\right)$ and $1.5 \%$ xanthan gum solution: (a) $z / H=0.20,(\mathbf{b}) z / H=$ 0.50 , and (c) $z / H=0.86$. 184

Figure (5.5-12). Dimensionless average shear rate at $R_{n}=8\left(N_{c}=240 \mathrm{rpm}\right.$ and $\left.N_{a}=30 \mathrm{rpm}\right)$ and $1.5 \%$ xanthan gum solution along a line parallel to the $z$-axis: (a) $2 r / T=0.25$, (b) $2 r / T=$ 0.45, (c) $2 r / T=0.558$, and (d) $2 r / T=0.68$. 186

Figure (5.5-13). Apparent viscosity (kg/m.s) contour plots at $R_{n}=8\left(N_{c}=240 \mathrm{rpm}\right.$ and $N_{a}=30$ rpm) and 1.5\% xanthan gum solution: (a) Scaba-Scaba-anchor coaxial (DSAC) mixer, (b) Rushton-Scaba-anchor coaxial (RSAC) mixer, (c) Scaba-Rushton-anchor coaxial (SRTAC) mixer, (d) Scaba-pitched blade-anchor coaxial (SPAC) mixer, and (e) pitched blade-Scabaanchor coaxial (PSAC) mixers. 188

Figure (5.5-14). Energy consumption for five coaxial mixers at $R_{n}=8\left(N_{c}=240 \mathrm{rpm}\right.$ and $N_{a}=$ $30 \mathrm{rpm}), 1.5 \%$ xanthan gum solution, and the co-rotating mode. 190 


\section{Chapter 1}

\section{Introduction}

The mixing of non-Newtonian fluids plays a major role in different industries such as biotechnology, food processing, chemical and petrochemical, cosmetic, water treatment, and polymer based manufacturing. The mixing of these fluids is a difficult task due to the complex rheology exhibited by the fluid during the mixing process. For a specific type of these fluids, called yield-pseudoplastic fluids, the fluid starts flowing when the shear stress exceeds the specified value (yield stress). This behavior is due to the existence of a network among the molecules of the fluid, which is built at the low shear rates and breaks down at the higher shear rates. Due to this rheological behavior two regions are formed during the mixing process: a well-mixed region around the impeller called cavern and a dead zone or almost stagnant areas at the rest of the fluid (Solomon, 1981; Zlokarnik, 2001; Pakzad et al., 2008a, b and c, 2013 b). The stagnant region results in weak mass and heat transfer, which is primarily responsible for the production of low quality 
products (Amanullah, 1997). Therefore, the elimination of the stagnant region is considered as a main target in the design of the agitated system for highly viscous yieldpseudoplastic fluids. In order to avoid the formation of these dead zones, an efficient mixing configuration should be selected. The effective mixing of such complex fluids demands the use of more advanced hybrid agitated system such as the planetary mixers or multi-shaft mixers (Rudolph et al., 2007; Bao et al., 2011; Pakzad et al., 2013b).

The performances of different mixing systems such as a single central impeller (Pakzad et al., 2008b), centered multiple agitators (Montante and Magelli, 2004), off-centered single agitator or eccentric agitator (Montante et al., 2006; Alvarez et al., 2002), dual shaft mixers (Khopkar et al., 2007), side-entering impellers (Ford et al., 2006), planetary mixers (Delaplace et al., 2007;Tanguy et al., 1999; Tanguy et al., 2001 ), conical mixers (Dubois et al., 1996), and large-diameter close clearance impellers like anchor (Triveni et al., 2009; Prajapati and Ein-Mozaffari, 2009; Savreux et al., 2007; Iranshahi et al., 2006; Murthy and Jayanti, 2003a \&b; Bertrand et al., 1996; Tanguy et al., 1996; Tanguy et al., 1994; Kaminoyama et al., 1994; Rubart and Bohme, 1991; Kaminoyama et al., 1990; Sestak et al., 1986; Ohta et al., 1985; Kuriyama et al., 1982; and Calderbank and Moo-Young, 1961; Pedrosa and Nunhez 2000; Prajapati and Ein-Mozaffari, 2009; Bertrand et al., 1996; Tanguy et al., 1996; Tanguy et al., 1994; Kaminoyama et al., 1994), in the mixing of highly viscous non-Newtonian fluids have been investigated either numerically or experimentally. These studies have demonstrated that multi shafts or nonstandard systems such as coaxial agitated systems, composed of a central impeller (e.g. a Rushton impeller rotating at a high speed), and an anchor rotating at a low speed can be a proper system for the agitation of the non-Newtonian fluids (Rudolph et al., 2007; Bao et 
al., 2011; Pakzad et al., 2013b). This configuration provides an opportunity for both impellers to rotate independently on the different shafts. This design combines the effectiveness of the high speed impellers suitable for low viscosity fluids and the anchor impeller suitable for high viscosity fluids.

Although the coaxial mixers are broadly used in industry such as biochemical processes in pharmaceutical and biotechnology industry (Bonnot et al., 2007), paper coating fluid preparation in paper industry (Foucault et a., 2006), and gellan production in polymer industry (Espinosa-Solares et al., 2006), the limited information can be found in the literature regarding the mixing of the highly viscous non-Newtonian fluids especially the yield-stress fluids with the coaxial mixers.

The majority of the above mentioned studies on the applications of the coaxial mixers have focused on the performance of the agitated systems comprised of one central impeller and one anchor in the mixing operation of non-Newtonian fluids. However, the hydrodynamic performance of the mixing system equipped with double central impellers (without anchor) in agitation of the Newtonian fluids in turbulent regime either numerically or experimentally has been explored by Jaworski et al. (2000), Bujalski et al. (2002), Montante et al. (2004), Mahmodi and Yianneski (1991), Rutherford et al. (1996), Mishra and Joshi (1994), Baudou et al. (1997), Mukataka et al.(1981), Fort et al. (1986), Taguchi and Kimura (1970), Komori and Murakami (1988), Hudcova et al. (1989), Cronin et al. (1994), Hiraoka (2001), Bates et al. (1963and 1966), and Magelli et al. (1988).

Although the mixing quality has been improved by using the coaxial mixers (Foucault et al., 2004, Pakzad et al., 2013 b), no information has been reported regarding the agitation 
of the non-Newtonian yield-pseudoplastic fluids by the coaxial mixers composed of an anchor impeller and two central impellers, either identical or different in shape.

Therefore, the main objective of this study was to explore the performance of a novel coaxial mixer composed of two central impellers and one close clearance impeller for the mixing of non-Newtonian yield-pseudoplastic fluids. To achieve this goal, the effects of the design parameters (e.g. impeller speeds, central impeller type, impeller spacing, fluid rheology, and speed ratio) on power consumption, mixing time, flow characterization, mixing efficiency, and mixing energy were assessed based on the mixing requirements in this study. Electrical resistance tomography (ERT) was applied to measure the degree of homogeneity and to visualize the flow inside the mixing tank. The three-dimensional flow domain was simulated using the advanced computational fluid dynamics (CFD) technique. To validate the CFD model, the simulation results were compared to the experimentally measured torques and mixing times. The validated CFD model was then utilized to obtain further information (i.e. the flow pattern, pumping capacity, and velocity profiles) for the new coaxial mixer. Design of experiments (DOE) and response surface methodology (RSM) were utilized to analyze the mixing data and to determine the optimal design parameters.

Chapter 2 is devoted to a comprehensive literature review of the coaxial mixing system, mixing systems equipped with double central impellers (without anchor) and the research objectives are presented.

Chapter 3 focuses on the experimental setup (including tomography system and DOE), the fluid rheology, and the experimental procedures and conditions. 
Chapter 4 describes the development of the CFD models for the coaxial mixing systems employed in this study.

Chapter 5 provides the experimental and CFD results with the thorough discussions. This chapter is divided into five sections as follows:

- Section 5.1 presents the effect of the rheological properties on the mixing of Herschel-Bulkley fluids with the coaxial mixers.

- Section 5.2 focuses on the hydrodynamic performances of coaxial Mixers in agitation of yield-pseudoplasitc fluids: single and double central impellers in combination with the Anchor.

- Section 5.3 Provides a new perspective in the evaluation of the mixing of biopolymer solutions with different coaxial mixers comprising of two identical dispersing impellers and a wall scraping anchor.

- Section 5.4 describes the effect of the impeller spacing on the flow field generated by the coaxial mixing system composed of double central impellers and an anchor in the agitation of yield-pseudoplastic Fluids.

- Section 5.5 mainly focuses on hydrodynamics of coaxial mixers with two different central impellers combined with the anchor in the agitation of biopolymer fluids.

Finally, Chapter Six summaries the major conclusions of this study and gives recommendations for the future work. 


\section{Chapter 2}

\section{Literature Review}

Many industrial applications such as polymerization, emulations, suspension, fermentation, food and polymer based manufacturing require mixing processes. During mixing operations, fluids exhibit a wide variety of different rheological behaviors ranging from Newtonian to non-Newtonian (Rao, 1999). Different types of impellers such as axial, radial, proximity impellers, or combination of them are employed for the agitation of fluids. The design of the mixing systems for the agitation of non-Newtonian yieldpsedoplastic fluids is a challenging task due to complex rheology exhibited by this type of fluids. This complex rheology creates many operational problems such as formation of gel or lumps, fouling and buildup on the walls, increasing mixing time and stagnant zones

during the mixing process (Rudolph et al., 2009; Bao et al., 2011). These problems affect the efficiency of mixing, which has significant effect on the product quality. 
This chapter is devoted to a comprehensive literature review of the coaxial mixers and mixing systems equipped with double central impellers (without anchor).

\subsection{Coaxial Agitated Systems}

The performances of different mixing systems such as a single central impeller (Pakzad et al., 2008b), centered multiple agitators (Montante and Magelli, 2004), off-centered single agitator or eccentric agitator (Montante et al., 2006; Alvarez et al., 2002), dual shaft mixers (Khopkar et al., 2007), side-entering impellers (Ford et al., 2006), planetary mixers (Delaplace et al., 2007;Tanguy et al., 1999; Tanguy et al., 2001 ), conical mixers(Dubois et al., 1996), and large-diameter close clearance impellers like anchor (Triveni et al., 2009; Prajapati and Ein-Mozaffari, 2009; Savreux et al., 2007; Iranshahi et al., 2006; Murthy and Jayanti, 2003a \&b; Bertrand et al., 1996; Tanguy et al., 1996; Tanguy et al., 1994; Kaminoyama et al., 1994; Rubart and Bohme, 1991; Kaminoyama et al., 1990; Sestak et al., 1986; Ohta et al., 1985; Kuriyama et al., 1982; and Calderbank and Moo-Young, 1961; Pedrosa and Nunhez 2000; Prajapati and Ein-Mozaffari, 2009; Bertrand et al., 1996; Tanguy et al., 1996; Tanguy et al., 1994; Kaminoyama et al., 1994) in the mixing of highly viscous non-Newtonian fluids have been investigated either numerically or experimentally. The results of these studies have demonstrated that the coaxial mixers are suitable for the mixing of highly viscous non-Newtonian fluids. A coaxial mixer is a combination of a central impeller (e.g. Scaba) rotating at a high speed, and a close-clearance impeller (e.g. anchor) rotating at a low speed. It provides an opportunity for both impellers to rotate independently on the different shafts. This design combines the effectiveness of the high speed impellers suitable for low viscosity fluids and anchor impeller suitable for high viscosity fluids. 
Generally, two approaches can be found in the literature for the assessment of the hydrodynamic performances of the coaxial mixers. In the first approach, the researchers have focused on developing the correlations for generalized Reynolds number and power number. This approach considers the effects of the geometrical and rheological parameters on the power consumption of the coaxial mixing system (Thibault and Tanguy, 2002; Kohler and Hemmerle, 2003; Foucault et al., 2004; 2005; Farhat et al., 2008; Rudolph et al., 2009; Bao et al., 2011; Pakzad et al., 2013c). All aforementioned researches resulted in obtaining the master power curves for the performance of the coaxial mixing systems for both Newtonian and non-Newtonian fluids. In the second approach, the mixing efficiency is mainly assessed by measuring the mixing time, which is defined as the time required to reach a specified degree of homogeneity (Schneider and Todtenhaupt, 1990; Pakzad et al., 2013a; Foucault et al., 2004, 2006; Tanguy et al., 1997; Espinosa et al, 1997). Our literature review revealed that limited information has been reported regarding the assessment of the performance of the coaxial mixers in the agitation of the yield-pseudoplastic fluids. The most recent study in this area was conducted by Pakzad et al. (2013 a\&b\& c and d) for the Scaba-anchor coaxial mixer and their goal was to develop new correlations for power number and Reynold number.

\subsection{Power Consumption of the Coaxial Mixing Systems}

Our literature review shows that few researchers have attempted to characterize the power consumption of the coaxial mixers. In this mixing configuration, due to the use of two different kind of impellers with distinct diameters and speeds, the definition of characteristic length $(D)$ and rotational speed $(N)$ for power number and Reynolds number is considered as a challenging task. The power number and Reynolds number are 
also affected by the rotating mode of the impellers (i.e. co-rotating and counter-rotating). Moreover, these dimensionless numbers also depend on the rheological parameters of the non-Newtonian fluids. Thus, the rheological parameters must be incorporated into the power and Reynolds numbers.

Schneider and Todtenhaupt (1990) studied a coaxial system composed of an anchor with a dual set of VISCOPRO (Ekato Ruhr- und Mischtechnik GmbH). They investigated the performance of the system through the evaluation of mixing time and heat transfer. They noticed that the anchor functioned as a baffle in the case of water while it had a major effect on the mixing for highly viscous fluids, and as a result the heat transfer was improved extremely inside the agitated system. However, authors did not provide any information regarding the rotating mode and power consumption of the system.

Tschuor and Widmer (1992) utilized a coaxial system consisting of two high speed impellers mounted on the same axis in the turbulent regime. The top impeller pumped the fluid downward while the bottom one was in the upward pumping mode. This combination prevented the formation of the vortex in non-baffled areas and reduced the mixing time.

Tanguy and Espinosa-Solares (1997) investigated the power consumption of a coaxial system, a combination of a Rushton turbine and a helical ribbon impeller, in the corotating mode. The results of their research showed that the mixing quality achieved by the coaxial system was better than that of the standard helical alone, but with a higher power consumption. Following this study, Espinosa-Solares et al. (1997) showed that the total power consumption of the co-axial system was not the summation of the individual impeller contribution. 
Thibault and Tanguy (2002) studied a coaxial system, which was a combination of an anchor with a series of rods positioned 90 degrees from one another and a pitched turbine with two blades located at the bottom of the tank. In this system, anchor and inner impellers were rotated in the counter-rotating mode. They concluded that the MetznerOtto (1957) concept could be applied by using the speed ratio of two impellers. They developed a single master curve for the power consumption, but they were not able to determine each impeller's contribution to total power consumption. With choosing speed and diameter of the anchor as the characteristic length and speed, the following generalized Reynolds and power numbers were developed:

$$
\begin{gathered}
N_{p}(\text { Coaxial })=\frac{P_{t o t}}{\rho\left(N_{a}\right)^{3}\left(D_{a}\right)^{5}} \\
\operatorname{Re}_{g}=\frac{\rho\left(N_{a}\right)^{2-n} D_{a}{ }^{2}}{K K_{R_{N}} K_{S}\left(R_{N}\right)^{n-1}}
\end{gathered}
$$

where $N_{p}, P_{t o t}, N_{a}, \rho, D_{a}, K_{R_{N}}, R e_{g}, K_{s}, K$ and $n$ are coaxial power number, coaxial power consumption, anchor rotational speed, fluid density, anchor diameter, shift factor, generalized Reynolds number, Metzner- Otto constant, consistency index, and power-law index constant, respectively.

Foucault et al. (2004) studied the performance of three coaxial systems composed of an anchor impeller and one of the Delfo, Sevin, and Hybrid as the central impeller in the mixing of Newtonian and non-Newtonian fluids. They realized that for both co-rotating and counter-rotating, the power consumption of the central impeller was not influenced 
by the speed of the anchor, while the anchor power was affected by the central impeller speed.

With focusing on the above mentioned systems, Foucault et al. (2005) initiated a research work to characterize the power consumption of the coaxial systems. For the first time, they proposed a characteristic parameter for both co-rotating and counter-rotating system as:

$$
\begin{gathered}
N_{\text {co-rotating }}=N_{c}+N_{a} \\
N_{\text {counter-rotating }}=N_{c}-N_{\boldsymbol{a}}
\end{gathered}
$$

where $N_{c}$ represents central impeller rotational speed. Then based on the suggested characteristic parameters and applying Rieger and Novak theory (1973), the following Reynolds and power numbers were proposed:

$$
\begin{aligned}
& N_{p}=\frac{P_{t o t}}{\rho\left(N_{c} \pm N_{a}\right)^{3}\left(D_{c}\right)^{5}} \\
& R_{e}=\frac{\left(N_{c} \pm N_{a}\right)^{2-n}\left(D_{C}\right)^{2} \rho}{K}
\end{aligned}
$$

where $D_{c}$ is the central impeller diameter.These correlations were applicable for the speed ratios higher than 10. Rivera et al. (2006) and Farhat et al. (2007) utilized successfully the correlations developed by Foucault et al. (2005). Later, Foucault et al. (2006) defined the Reynolds number for both co-rotating and counter-rotating modes by applying the concept of Metzner-Otto for Newtonian and power law fluids as follows: 


$$
R_{e}=\frac{\left(N_{c} \pm N_{a}\right)^{2-n}\left(D_{c}\right)^{2} \rho}{K K_{s(\text { central impeller })}^{n-1}}\left\{\begin{array}{lr}
N_{\text {co-rotating }}=N_{c}+N_{a} \\
N_{\text {counter-rotating }}=N_{c}-N_{a} \\
n=1: & \text { Newtonian }
\end{array}\right.
$$

In 2007 Rudolph conducted a research work to characterize the power consumption of a coaxial mixer consisting of an anchor with a dual set of the pitched blade turbine. They developed new generalized Reynolds number and power number for power-law fluids assuming anchor rotational speed and diameter as a characteristics parameters as:

$$
\begin{gathered}
N_{p}=\frac{P_{t o t}}{\rho N_{a}{ }^{3} D_{a}{ }^{5}} \\
\operatorname{Re}=\frac{\rho N_{a}{ }^{2-n} D_{a}{ }^{2}}{K K_{t r}\left(K_{S t o t}\right)^{n-1}} \\
K_{t r}=e^{N_{c} D_{c}}
\end{gathered}
$$

where, $K_{t r}, t_{r}$ and $a_{4}$ are shift factor, tip speed, and a constant. In this study, The Reynolds number was the Reynolds umber of the anchor divided by a shift factor $K_{t r}$. Their results showed good agreement with those reported by Thibault and Tanguy (2000).

Farhat et al. (2007) focused on the power consumption and mixing time of two coaxial systems: an axial impeller with an anchor and a radial impeller with an anchor. Their investigation proved that the efficiency of the system using axial mixer was better than that of the Rushton turbine with anchor. They also found that the efficiency of the corotating system was higher than that of the counter-rotating coaxial system. After this 
study, Farhat et al. (2008) conducted another research on the performance of a coaxial system composed of a Rushton turbine with an anchor. The coaxial system operated in laminar and transitional flow in the co-rotating mode. They defined a new characteristic speed for co-axial mixers as follows:

$$
N^{\prime}=\frac{N_{c} D_{c}+N_{a} D_{a}}{D_{c}}
$$

This new characteristic parameter was applicable for all mixing modes, and thus the Reynolds number and power number were modified as follows:

$$
\begin{array}{r}
\mathrm{N}_{\mathrm{p}}=\frac{\mathrm{P}_{\text {tot }}}{\rho\left(\mathrm{N}_{\mathrm{c}} \mathrm{D}_{\mathrm{c}}+\mathrm{N}_{\mathrm{a}} \mathrm{D}_{\mathrm{a}}\right)^{3} \mathrm{D}_{\mathrm{c}}{ }^{2}} \\
\operatorname{Re}=\frac{\left(N_{c} D_{c}+N_{a} D_{a}\right) D_{c} \rho}{\mu}
\end{array}
$$

A single master power curve was then generated, which was applicable to all rotating mode. Bao et al. (2011) worked on the coaxial agitated system and realized that the influence of the anchor on the total power consumption at the higher speed ratio became weaker, and thus the authors proposed the new correlations for the power number and Reynolds:

$$
N_{p}=\frac{P_{t o t}}{\rho\left(N_{c} \pm \frac{N_{a}}{R_{n}}\right)^{3} D_{c}{ }^{5}}
$$




$$
\left\{\begin{array}{l}
N_{c}+\frac{N_{a}}{R_{n}}: \text { conter }- \text { rotating } \\
N_{c}-\frac{N_{a}}{R_{n}}: \quad \text { co }- \text { rotating }
\end{array}\right.
$$

$$
R e=\frac{\left(N_{c} \pm \frac{N_{a}}{R_{n}}\right)^{3} D_{c}^{2} \rho}{K K_{s}^{n-1}}
$$

Here, $N_{p}$ is the coaxial power number; $n$ is the power-law index; $K$ is the consistency index; $P_{\text {tot }}$ is the total power consumption of coaxial system; $\rho$ is the fluid density; $N_{c}$ is the rotational speed of the central impeller; $R_{n}$ is the speed ratio; $N_{a}$ is the anchor rotational speed; $D_{c}$ is the central impeller diameter; and $( \pm)$ are positive and negative signs represent the co- or counter-rotating regimes, respectively.

Pakzad et al. (2013b) assessed the performances of three coaxial systems (anchor-Scaba, anchor-Rushton, and anchor-ARI) in the mixing of yield-pseduplastic fluids for both rotating modes. They modified the previous correlations for this type of fluid, and none of them was able to predict accurately the effects of all operating conditions on the power number of the coaxial mixer as a function of the Reynolds number. Therefore, they introduced new correlations for generalized Reynolds and power number. They incorporated the anchor power fraction $\left(f p_{(a)}=P_{a} / P_{\text {tot }}\right)$ into power number and Reynolds number as follows:

$$
N_{p}=\frac{P_{t o t}}{\rho\left(N_{c}+f_{p(a)} N_{a}\right)^{3} D_{s}^{5}}
$$




$$
R e=\frac{K_{S(s)}\left(N_{c}+f_{p(a)} N_{a}\right)^{2} D_{s}{ }^{2} \rho}{\left[\tau_{y}+K\left(K_{s(c)}\right)^{n}\left(N_{c}+f_{p(a)} N_{a}\right)^{n}\right]}
$$

where $N_{P(\text { coaxial })}$ is the coaxial power number; $P_{\text {tot }}$ is the coaxial power consumption; $\rho$ is the fluid density; $N_{c}$ is the rotational speed of the central impeller; $N_{a}$ is the rotational speed of the anchor; $D_{c}$ is the central impeller diameter; $f_{p(a)}$ is the anchor power fraction; and $K_{S(c)}$ is Metzner and Otto constant (1957) for the central impeller.

In most of the abovementioned studies, a coaxial system composed of a single central impeller and an anchor was used for the agitation of Newtonian and non-Newtonian shear thinning fluids. The only study on the mixing of yield-pseduplasic fluids was conducted by Pakzad et al. (2013a, b and c). To our knowledge, no research has been done regarding the combination of a close clearance impeller and two central impellers for the agitation of the fluids with complex rheology such as yield-pseudoplastic fluids. Therefore, more studies should be conducted in this field.

\subsection{Double Impellers Agitated Systems}

Multi-impeller systems have been used widely in industries. They promote their own advantages such as better and easier heat removal, better gas utilization when using sparing gas, less variable shear rate in the stirred liquid, and more compact equipment. The agitated system equipped with double impellers mounted centrally on a shaft creates the complexity for the mixing system where different types of impellers are utilized. The performance of such a system can be determined by flow characteristics of the employed impellers and the interaction between the impellers streams (Rutherford et al., 1996). Usually for double impeller systems, the use of large impeller diameters and large off- 
bottom clearance is suggested. In these conditions, there is a little or no interaction between flows generated by individual impellers (Kuboi and Nienow, 1982). The intensity of interaction between impeller discharge flows depends on the space between the mounted impellers on the central shaft. For impeller spacing equal to one-half of the tank diameter, the impellers act independently with a little interaction between them. The interaction increases with a decrease in impeller spacing, which might lead to the complicated and unstable flow pattern.

In a stirred multiple-impeller system, the distance between two impellers and off-bottom clearance of impeller have a significant impact on the mixing performance of the agitated system. If the impeller spacing and the bottom clearance of impeller are set well without significant interaction between them, then, the generated flow by each of them would be similar to a single impeller (Smith, 1987; Bouaifi et al, 1997; Hiraoka et al; 2001). Literature review shows that a variety of investigations have been conducted on the utilization of the different impeller combinations either identical or different impellers for the agitated systems. Mahmodi and Yianneski (1991) and Rutherford et al. (1996) studied the effect of impeller spacing on the performance of a multiple-impeller system equipped with double Rushton turbines. They noticed a decreased trailing vortex structure and flow periodicity for the impeller spacing equal to one-third of the tank diameter or smaller. In such a condition the region between the turbines was anisotropic. Therefore, their research led to the identification of three flow patterns in a double-impeller system: parallel, diverging and merging flow. The similar study was carried out by Mishra and Joshi (1994), but they used identical Rushton turbines or a combination of a Rushton turbine and a pitched-blade turbine. Also, Baudou et al. (1997) worked on the similar 
research, but their focus was on axial impellers. The effect of the impeller spacing on the liquid circulation was also investigated by Mukataka et al. (1981) and Fort et al. (1986), where they showed that the liquid circulation was affected significantly by the impeller spacing.

Power consumption is considered as a key parameter for the design of mixing systems. The power consumption is a function of the rotational speed, type and number of impellers, physical properties of fluid, and geometry of the system including dimension and location of impeller in the stirred system. There is plenty of information in literature for power consumption of single impeller while limited data have been reported for the multiple impellers. There is also less information regarding the power dissipation of a single impeller in multiple-impeller systems. In a double-impeller system, the number of impellers and off-bottom clearance of lower impeller influence the performance of the system. In this system, the second impeller reduces the fillet region near the tank, and makes the flow at the bottom of the tank more parallel to the bottom of the tank. The power consumption by each impeller in a multiple-impeller system can be lowered (as little as $48 \%$ and as high as $84 \%$ ) compared with the single impeller system. This strongly depends on the position of the lower impeller relative to the tank (Armente, et al., 1999). Basically, the information related to the single impeller system can be used to evaluate the optimal number and location of the impellers to achieve mixing objectives with the power consumption reduction.

The effect of the impeller spacing on the power consumption of the multiple-impeller system was investigated by Taguchi and Kimura, 1970; Komori and Murakami, 1988; Hudcova et al., 1989; Cronin et al., 1994; Hiraoka 1988; Hudcova et al., 1989; Hidaka et 
al., 2001 and Bates et al. (1963, 1966). They found that the power consumption of a multiple-stirrer system was affected by the impeller spacing. The same research was conducted on the assessment of the mixing time by Mukataka et al. (1981), Magelli et al., 1986, Komori and Murakami (1988), and Cronin et al. (1994). They found that the mixing time strongly depends on the impeller spacing. Mahmudi et al. (1994) and Rutherford et al. (1992) studied the effect of impeller thickness $\left(t / D_{0}\right)$ on the power consumption, flow number, mixing time, and turbulent level of the single and double Rushton impellers.

The homogenization achieved by the double Rushton impellers was investigated by Jaworski et al. (2000), Bujalski et al. (2002), and Montante et al. (2004) through different simulation techniques. The result showed that the computed mixing times considering different impeller spacing were about two to three times longer than the measured values. Later Montante et al. (2004) focused on modified CFD model in order to improve the agreement, and they could predict the effect of the impeller spacing on the degree of homogenisation.

The abovementioned researches demonstrate that many studies have been conducted to study the performance of the agitated systems equipped with the multiple central impellers for the agitation of Newtonian fluids in the turbulent regime. Most of these studies have focused on the combination of the identical Rushton or pitched blade turbines alone rather than the combination of the different types of impellers. No research has been conducted on the agitation of the fluids with complex rheology with a coaxial mixer composed of a close clearance impeller and two central impellers. Therefore, more studies should be conducted in this field. 


\subsection{Mixing Time}

Mixing time is an essential parameter in the assessment of the performances of the mixing systems (Pakzad et al., 2008c, Parajapati et al., 2009). Mixing time is defined as the time needed to achieve a certain degree of homogeneity after the addition of the second fluid (called tracer) to a mixing system. Mixing time depends on the type of impeller and operating conditions. To determine the mixing time, several physical and chemical methods have been developed. Generally, the small amount of the tracer is added into the agitated system to measure the degree of homogeneity. Based on the temporal change of tracer concentration, the mixing quality is defined with a dimensionless correlation (Landau, 1961) as follow:

$$
M(t)=\frac{m_{0}-m_{(t)}}{m_{0}-m_{\infty}}
$$

where $m_{0}$ is the initial mass fraction, $m_{\infty}$ is the final mass fraction of tracer after infinite time. Usually the mixing time is reported for $90 \%, 95 \%$, or $98 \%$ of homogeneity. It means that at any operating condition, the mixing time is defined as time when the tracer concentration profile at all monitoring points reaches the equilibrium concentration value, and remains within $10 \%, 5 \%$, or $2 \%$ of this steady state value (Mavros et al., 2001).

The most common methods for measuring the mixing time are: $\mathrm{pH}$ or conductivity probes (Bouaifi and Rouston 2001; Shiue and Wong, 1984; Sano and Usui; 1985), dye discoloration method (Moo-Young et al., 1972; Brennan and Lohrer, 1976), colored dye addition method (Ascanio et al.2002), the utilization of probes temperature (Rewatkar and Joshi, 1991), Laser-induced Fluorescence (Distelholff et al., 1990), radioactive liquid tracer (pant et al., 2001). Some of these methods are based on visualization, and they are 
not applicable to opaque fluids such as xanthan gum solution. The probe techniques have their own disadvantages such as affecting the flow inside the system. Also, they provide limited data restricted by few monitoring points inside of the system. It is obvious that providing such a data supports poorly understanding of agitated area, which is critical to the quality of the end product (pakzad et al., 2007).

In order to visualize inside of the process, scientists and engineers have developed a system, which is called tomography method. It is run based on the development of imaging process. This technique is categorized as resonance, electrical, or acoustic (ultrasonic). The most popular method is called electrical resistance tomography (ERT), which produces the cross-sectional images of the mixing system through a non-intrusive approach. This method monitors the conductivity variations inside the tank after the injection of the second liquid (tracer). The advantages of using ERT system compared with other methods are low cost, non-intrusive technique and applicability to opaque fluids. Tomography technique has been used for the characterization of the different processes such as drainage of porous media (Fordham et al., 1993), two-phase pipe flow (Huang et al., 1992), trickling filters (Toye et al., 1994), and stirred vessel mixing (Mann et al., 1997; Holden et al., 1998; Vlaev et al., 2000; Haibo and Wang 2006; Razzak et al., 2007; Pakzad et al. 2008\& 2013a\&d, Zhao et al., 2008, Hosseini et al., 2010), investigation of mixing operations (Kim et al., 2003), monitoring of stability of reaction in polymerization reactor (Kaminoyama et al.,, 2005), investigation of solid-liquid filtration operation (Vlaev et al., 2000), examination of a hydro cyclone (Williams, et al., 1999), measurement and control of bubble columns (Wange et al., 2001, Fransolet, et al., 2005), monitoring multiphase process such as liquid-solid (Lucase et al., 1999, Ricardo 
2005), gas-liquid (Wang 2001, Wang 2000) and liquid-liquid (Kaminoyama et al., 2007, Holden et al., 1998). Moreover, this technique can also be used to investigate the hydrodynamic performance of mixing system, which is critical for the mixing of nonNewtonian fluids.

Some empirical correlations have been developed by the researchers to evaluate the mixing time. For example, Prochazka and Landau (1961) proposed the correlations for mixing time in the turbulent regime based on the ratio of impeller and tank diameter. Moo-Young et al. (1972) studied the mixing time for both Newtonian and nonNewtonian fluids agitated with the paddle and turbine impellers. Shinue and wong (1984) developed correlations for the mixing of fluids in transitional and turbulent regimes. They found that the axial-flow impellers were more efficient than the radial-flow impellers. Sano and Usui (1985) proposed the correlations for the mixing time measured for the turbines and paddle impellers with vertical blades. Ruzkowski (1994) introduced the correlations for Rushton turbine based on the number of turbine impellers with different geometries. All developed correlations have their own applicability restrictions.

\subsection{Flow Pattern}

The mixing process is characterized by the power consumption of the impeller. The measured power consumption is an indicator of the energy dissipation within the agitated tank. In such a system, a three-dimensional flow field is generated by the impeller rotation, and the dissipation energy depends on the type of impeller and flow pattern created by the impeller. Therefore, the efficiency of the mixing system is influenced by flow patterns. These flow patterns depend on the different parameters such as rotational speed of the impellers, the geometry of the impeller, the number of the impeller, spacing 
between impellers, and the position of the impeller in the vessel, presence of baffle or other internals, and rheology of the fluid (Chhabra and Richardson, 1999). Figures [(2.51), (2.5-2), and (2.5-3)] depict the radial flow, axial flow, proximity flow, and the flow patterns generated by double impellers, respectively.

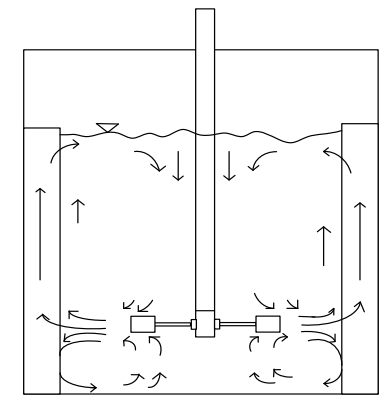

(a)

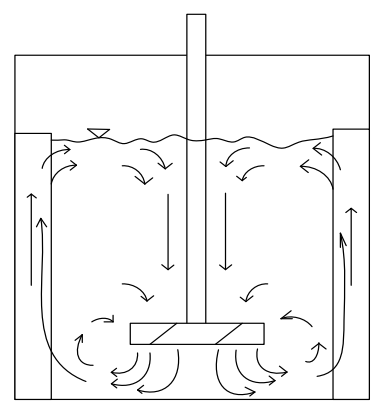

(b)

Figure (2.5-1). Flow patterns generated by: (a) a radial-flow impeller and (b) an axialflow impeller (Source: Zlokarnik, 2001).

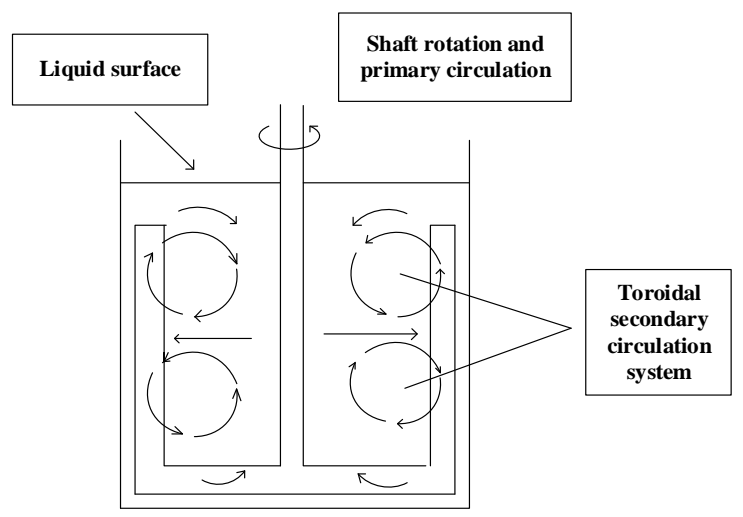

Figure (2.5-2). Flow pattern generated by an anchor impeller (Source: Taterson, 1991). 


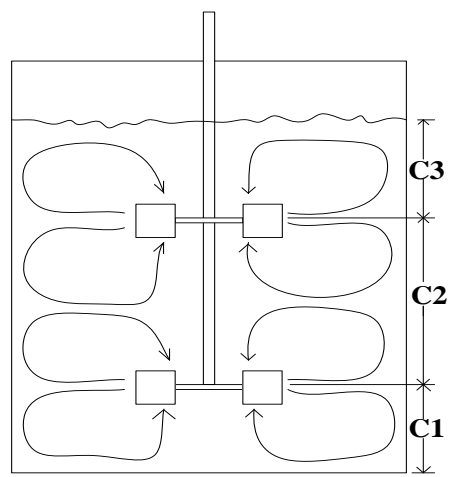

(a)

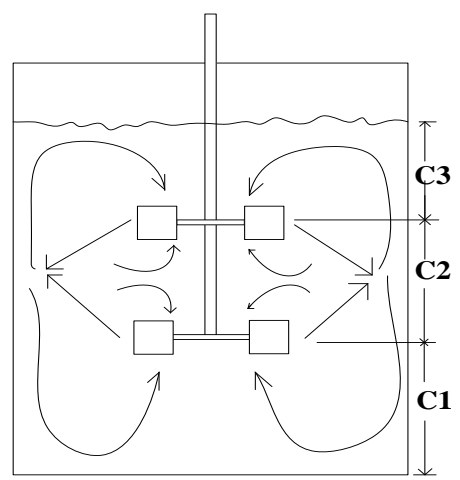

(b)

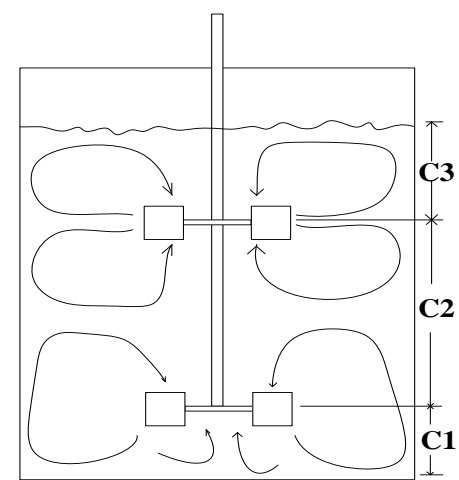

(c)

Figure 2.5-3. Stable flow patterns generated in a dual-Rushton turbine stirred vessel:

(a) Parallel flow; (b) merging flow; (c) diverging flow (Source: Rutherford et al., 1997).

Coaxial mixers have been proposed for the mixing of highly viscous fluids, and comprises a close-clearance impeller such as anchor and a central impeller. This combination provides good flexibility and efficiency. Literature review shows that there is limited studies on the flow pattern of the coaxial system with a single central impeller. The flow pattern generated by a coaxial system depends on the flow patterns generated by the central high-speed impeller (radial or coaxial) and anchor. In addition, both rotation modes either co-rotating or counter rotating can influence the generated flow pattern for a coaxial system. The flow pattern of a coaxial mixer composed of an anchor and a central impeller (i.e. Rushton or Mixel TT) in the mixing of the Newtonian and non-Newtonian fluids for both modes (co- and counter-rotating) has been investigated by Rivera et al. (2006), Farhat et al. (2007), and Bonet et al. (2007 ). They showed that well known radial-flow pattern is more dominant using the anchor impeller in co-rotating mode. In counter-rotating mode, there is a shift in the position of the center of this flow pattern, which is located closer to the central impeller. This character causes the size of 
the well mixed region which is smaller in the counter-rotating mode in compare with the co-rotating mode. On the other hand, they found more angular motion in both operation modes but a secondary circulation zone can be observed around the anchor when the impellers agitated in the counter-rotating mode.

Our comprehensive literature review shows that so far no research has been conducted on the analysis of the flow pattern created by a coaxial mixer composed of an anchor and two identical/different central impellers in the agitation of the non-Newtonian yieldpseudoplastic fluids. Therefore, more studies should be conducted in this field.

\subsection{Research Objectives}

In fact, an exhaustive search of the literature shows that very limited research has been devoted to study the mixing of highly viscous fluids with complex rheology with the coaxial mixer. Even among these few studies, less attention has been given to the agitation of the yield-pseudoplastic fluids. According to the author's knowledge, no attempt has been made to explore the performance of the coaxial mixers consisting of a close clearance impeller and two central impellers in the mixing of yield-pseudoplastic fluids. Therefore, the main objective of this study was to characterize the performance of this novel coaxial mixer in the agitation of the fluids with complex rheology. In this study, electrical resistance tomography and computational fluid dynamics (CFD) were employed to assess the mixing performance of this novel coaxial mixer as a function of different design parameters and operating conditions both experimentally and computationally. Design of experiment (DOE) and response surface methodology (RSM) were used to determine number of tests and to analyze the data. 
The goals of this study were:

- To analyze the flow domain generated by the novel coaxial mixing configuration.

- To develop a CFD model for this new coaxial mixer and validate the model with the experimental data.

- To investigate the effect of the rheological parameters on the hydrodynamic performance of the new coaxial mixer through experiments and CFD modeling.

- To determine the effect of central impellers type, impellers speed, speed ratio, fluid rheology, and impeller spacing on the hydrodynamic performance of the coaxial mixer composed of an anchor and double central impellers (identical/different impellers) in regard to the power consumption, mixing time, fluid characterization, and mixing energy.

- To develop a new correlation for the Reynolds number of the coaxial mixers composed of an anchor and two different types of the central impellers.

- To investigate the effect of the impeller spacing on the flow field generated by a coaxial mixer composed of double central impellers and an anchor in the mixing of yield-pseudoplastic fluids. 


\section{Chapter 3}

\section{Experimental Set-up and Procedure}

In this section, experimental set-up, fluid rheology, experimental procedure, and experimental conditions are described.

\subsection{Experimental Set-up, Material, and Methodology}

The experimental set-up is illustrated in Figure (3.1-1). The mixing tank was a cylindrical tank with an inner diameter, $T$, of $0.4 \mathrm{~m}$ and an aspect ratio $(H / T)$ of 1.25 providing a total volume of about $0.06 \mathrm{~m}^{3}$. The fluid inside the tank was agitated with a coaxial mixer, which was a combination of two central impellers and close clearance anchor. The tank was equipped with two shafts: one top entering shaft for the rotation of the high speed central impellers and one bottom entering shaft for the rotation of the anchor. Both shafts were rotated independently, and thus the impellers could be rotated in different rotating modes: co-rotating and counter-rotating and speeds. The top entering and bottom 
entering shafts were connected to a 1.5-hp direct-drive motor and a 1.0-hp gear-drive motor, respectively. Two independent solid-state frequency inverters (AC Technology Corporation, USA) were used to change the rotational speeds of the motors. Each shaft was equipped with a rotary torque sensor (S. Himmelstein and Company, USA) with two flexible couplings at each end to continuously measure the torque. A tachometer (DT205LR, Shimpo Instruments) was used to measure the rotational speed of the central impellers as well as the anchor. The configurations employed in this study were (Figure( 3.1-2)): a single or double central (identical or different) of the Scaba 6SRGT (a radial flow impeller), the Rushton turbine (a radial-flow impeller), and the pitched blade turbine (an axial-flow impeller) with $45^{\circ}$ pitched angle with a $0.18 \mathrm{~m}$ diameter $\left(D_{c}\right)$ in combination with an anchor impeller of diameter of $0.36\left(D_{a}\right)$, width of $0.036 \mathrm{~m}$ $\left(W_{a}\right)$, and height of $0.46 m\left(H_{a}\right)$, which was centrally attached to the bottom shaft with the bottom and tank wall clearance of $\left(C_{a}\right) 0.02 \mathrm{~m}$. 

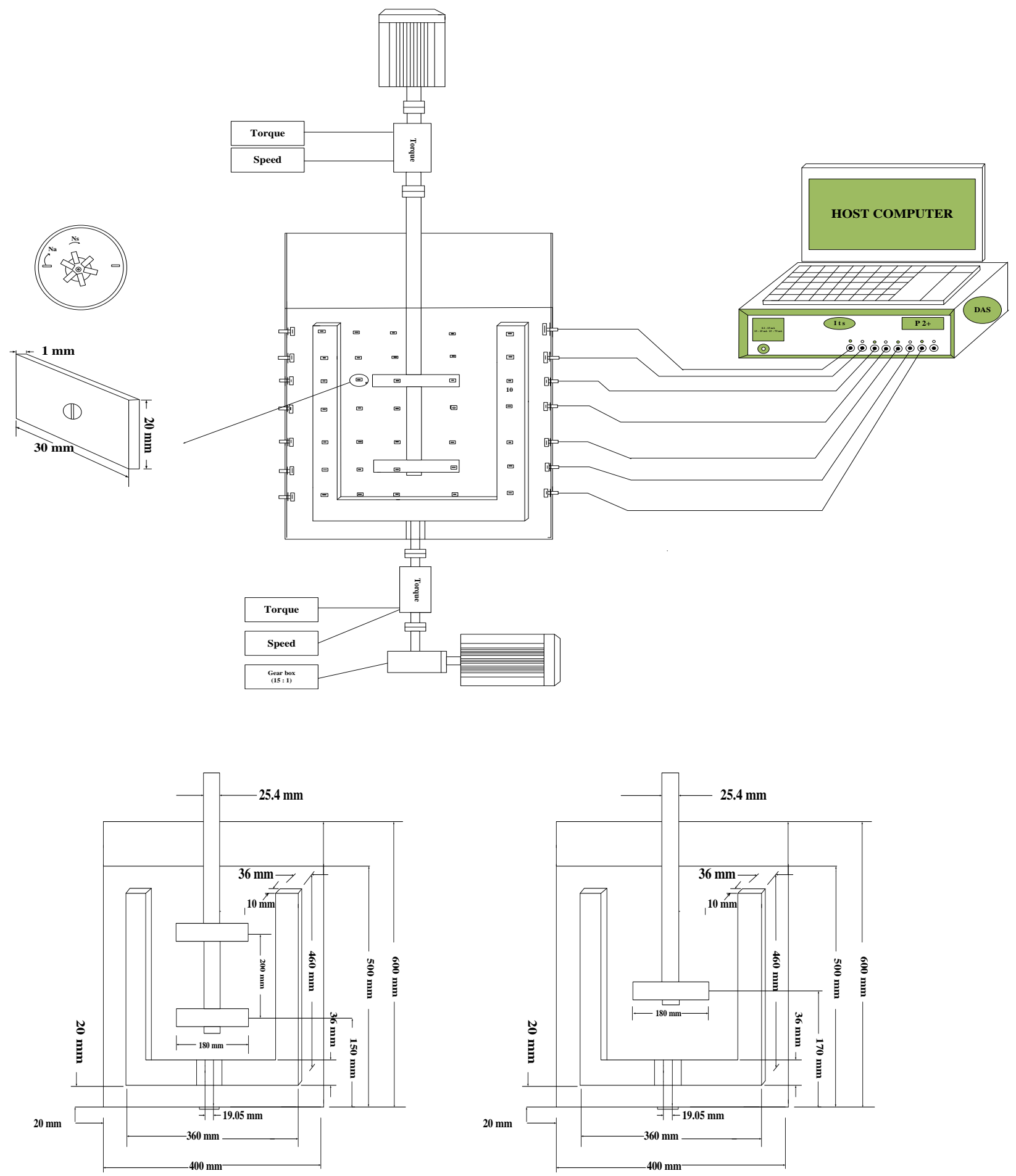

Figure (3.1-1). Schematic diagram of experimental setup. 

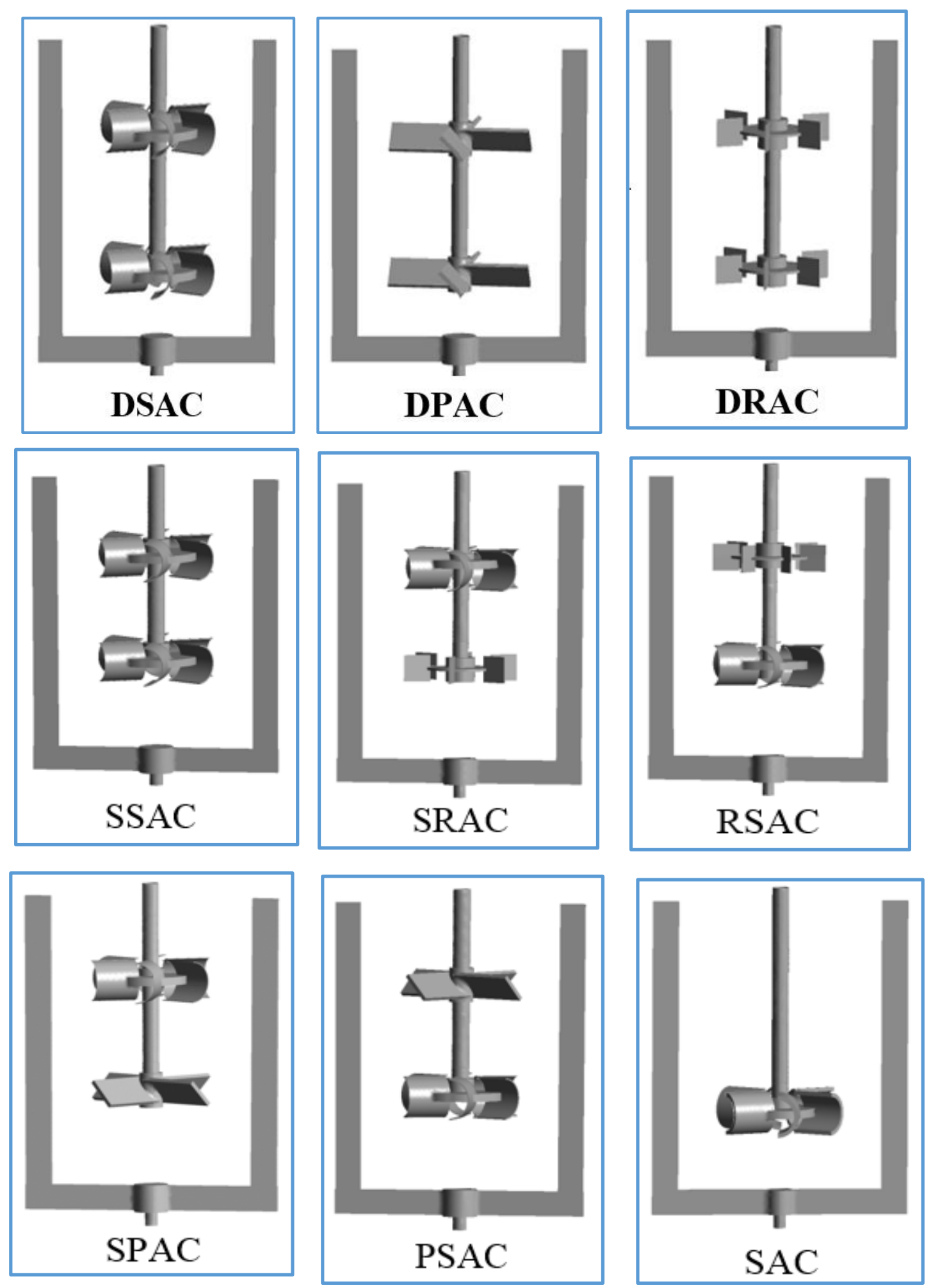

Figure (3.1-2). Coaxial configurations in this study. 


\subsection{Electrical Resistance Tomography (ERT)}

The mixing tank depicted in Figure (3.1-1) was equipped with seven tomography planes. Each plane comprised of 16 rectangular electrodes equally spaced around the periphery of the vessel. The thickness, width, and the height of each electrodes were $1 \mathrm{~mm}, 30 \mathrm{~mm}$, and $20 \mathrm{~mm}$, respectively. The conductivity distribution within the tank was measured by applying current on a neighboring pair of electrodes and measuring the voltages for the remaining pairs of electrodes mounted non-invasively on the inner wall of the tank through the data acquisition system (DAS). Applying the current and measuring the voltages were repeated for all adjacent electrodes of each plane. This measurement technique is called adjacent protocol (Barber et al., 1984).

Finally, DAS sent the quantitative data collected on the boundary of the tank to the host computer (Pentium 4, CPU 2GHz, and $512 \mathrm{MB}$ of Ram). These data were processed by applying a suitable image reconstruction algorithm. Generally, two kinds of algorithm are used for the image reconstruction: (1) non-iterative linear back projection algorithm (Barber and Brown 1984; Madupu et al., 2005) and (2) iterative sensitivity conjugate gradient (Wang 2002). The linear back projection algorithm was used in this study because the iterative algorithm is time consuming and slow for the real-time image reconstruction. ITS "ERTWIN" software (Industrial Tomography Systems- ITS, Manchester, UK) was employed for control, image reconstruction, and data storage.

The tomography machine utilized in this study was P2+ -v 8.0 - ERT system (industrial Tomography system (ITS), Manchester, UK). The frequency range, injection current range, output voltage range, and voltmeter sensitivity of the DAS in this study were: 75$153.6 \mathrm{kHz}, 0-75 \mathrm{~mA}$ (peak-peak), $-10 \mathrm{~V}$ (pp) to $+10 \mathrm{~V}$ (pp), and $0.0488 \mathrm{mV}$, 
respectively. The special resolution of the images in ERT system was around 2-5\% of the diameter sensor, using 16 equispaced electrodes (Holden et al., 1998).

\subsection{Fluid Rheology}

In this study, the xanthan gum powder (NovaXan, ADM, USA) was dissolved in water to prepare the solutions with the concentrations of $0.5,1.0,1.5,2$ and $2.5 \%$ as working fluids. The xanthan gum solution is a yield-pseudoplastic fluid and its rheological behavior is described by the Herschel- Bulkley (1926) model (Saeed and Ein-Mozaffari; 2008, Pakzad et al., 2008a, 2008b, and 2008c):

$$
\eta=\frac{\tau_{y}}{\dot{\gamma}}+K|\dot{\gamma}|^{n-1}
$$

where $\gamma$ is shear rate, $\tau_{y}$ is yield stress, $K$ is called consistency index, and $\mathrm{n}$ is the flow behavior index called power-law index. Table (3.3-1) summarizes the rheological characteristics of the xanthan gum solutions for different concretions (Pakzad et al., 2008a, and 2008b).

Table (3.3-1). Rheological properties of xanthan solutions.

\begin{tabular}{|c|c|c|c|c|c|}
\hline $\begin{array}{c}\text { Xanthan } \\
\text { Concentration } \\
(\%)\end{array}$ & $\begin{array}{c}\text { Consistency } \\
\text { Index, K } \\
\left(\mathrm{Pa} s^{n}\right)\end{array}$ & $\begin{array}{l}\text { Power-law } \\
\text { Index, n } \\
(-)\end{array}$ & $\begin{array}{c}\text { Yield Stress, } \\
\qquad \tau_{y} \\
(\mathrm{~Pa})\end{array}$ & $\begin{array}{c}\text { Density } \\
\rho \\
\left(\mathrm{Kg} / \mathrm{m}^{3}\right)\end{array}$ & $\begin{array}{c}\text { Yield } \\
\text { Viscosity } \\
\mu_{\circ} \\
(\mathrm{Kg} / \mathrm{m} . \mathrm{s})\end{array}$ \\
\hline 0.5 & 3 & 0.11 & 1.789 & 997.36 & 13.30 \\
\hline 1.0 & 8 & 0.12 & 5.254 & 991.80 & 22.61 \\
\hline 1.5 & 14 & 0.14 & 7.455 & 989.76 & 32.30 \\
\hline 2.0 & 19 & 0.15 & 11.687 & 988.00 & 44.15 \\
\hline 2.5 & 28 & 0.16 & 14.328 & 988.00 & 53.67 \\
\hline
\end{tabular}




\subsection{Design of Experiments (DOE)}

Design of experiments (DOE) or experimental design, as a systematic method, is used where a wide range of variations is noticed for process variables. This method helps to understand and determine the relationship between factors affecting output of the mixing process. It reduces the number, time, and cost of experiments. It also provides an opportunity to analyze the data and to assess the effect of different variables in order to optimize the output of the mixing process. In this study, Box-Wilson model (Box-Wilson, 1951) known as the central composite design (CCD) and Box-Behnken (Box and Behnken, 1960) design combined with the response surface methodology (RSM) (Ray et al., 2009) were employed. The Box-Wilson model, called central composite design (CCD), is widely used for five-level fractional factorial level to calculate the second order of the response surfaces. The main core of this design is the combination of the factorial or fractional factorial and central points. For the calculation of curvatures, the central points are combined with axial (star) points $(\propto$ ), which generate physical lower and upper limits for all factors. The distance from the center of design to fractional point and star point are shown with $\mp 1$ and $\pm|\propto|$, respectively (Myers and Montogomery, 2002). The Box-Behnken experimental design (BBD) requires three levels of each factor which are coded as $-1,0$, and 1 to run an experiment.

\subsection{Power Consumption Measurement}

Each shaft was equipped with a rotary torque sensor (S. Himmelstein and Company, USA) with two flexible couplings at each end to continuously measure the torque. The rotational speeds of the impellers were measured using a tachometer (DT- 205LR, Shimpo Instruments, USA). The bearing friction for the rotating shafts was measured by 
rotating the impeller in an empty tank. To determine the impeller power consumption, the friction torque was subtracted from the measured torque. To estimate the impeller power consumption, the friction torque was subtracted from the measured torque. Then the following equations were used to calculate the impeller power consumption:

$$
\begin{gathered}
M=M_{m}-M_{r} \\
P=M \omega=2 \pi N M
\end{gathered}
$$

where $M$ is corrected torque; $M_{m}$ is measured torque; and $M_{r}$ is residual torque, $P, \omega$, and $N$ are power consumption, the angular speed of the shaft, and agitation speed, respectively.

\subsection{Mixing Time Measurement}

For ERT system, the data collection and image reconstruction were made online. Prior to collecting data, the instrument current source and gain map were calibrated. The referenced state was taken to eliminate the effect of the stirrers and other internal structures. To measure the mixing time, $50 \mathrm{ml}$ of $10 \%$ saline solution, prepared by dissolving table salt in distilled water, was injected as the tracer $10 \mathrm{~cm}$ below the liquid surface on plane 1 using a plastic syringe. It has been reported the rheology of the xanthan gum solution changes when the concentration of the salt in the solution is greater than $0.17 w t / w t \%$ (Saeed and Ein-Mozaffari, 2008). Therefore, each batch of the xanthan gum solution was used few times until the amount of the salt reached approximately $0.17 \%(w t / w t)$. The injection should be fast enough for all experiments. The mean concentration of the salt or the conductivity of the fluid for each tomography plane was 
measured as a function of time through tomography machine. The tomography data were utilized to reconstruct the tomograms by using the linear back projection algorithm. These tomograms were used to determine the mixing time. The mixing time was defined as the time required for the concentration or conductivity to reach $95 \%$ of the steady state value as presented in Figure (3.6.1). Each test was repeated three times and the maximum standard deviation (less than 5\%) recorded for the mixing time values.
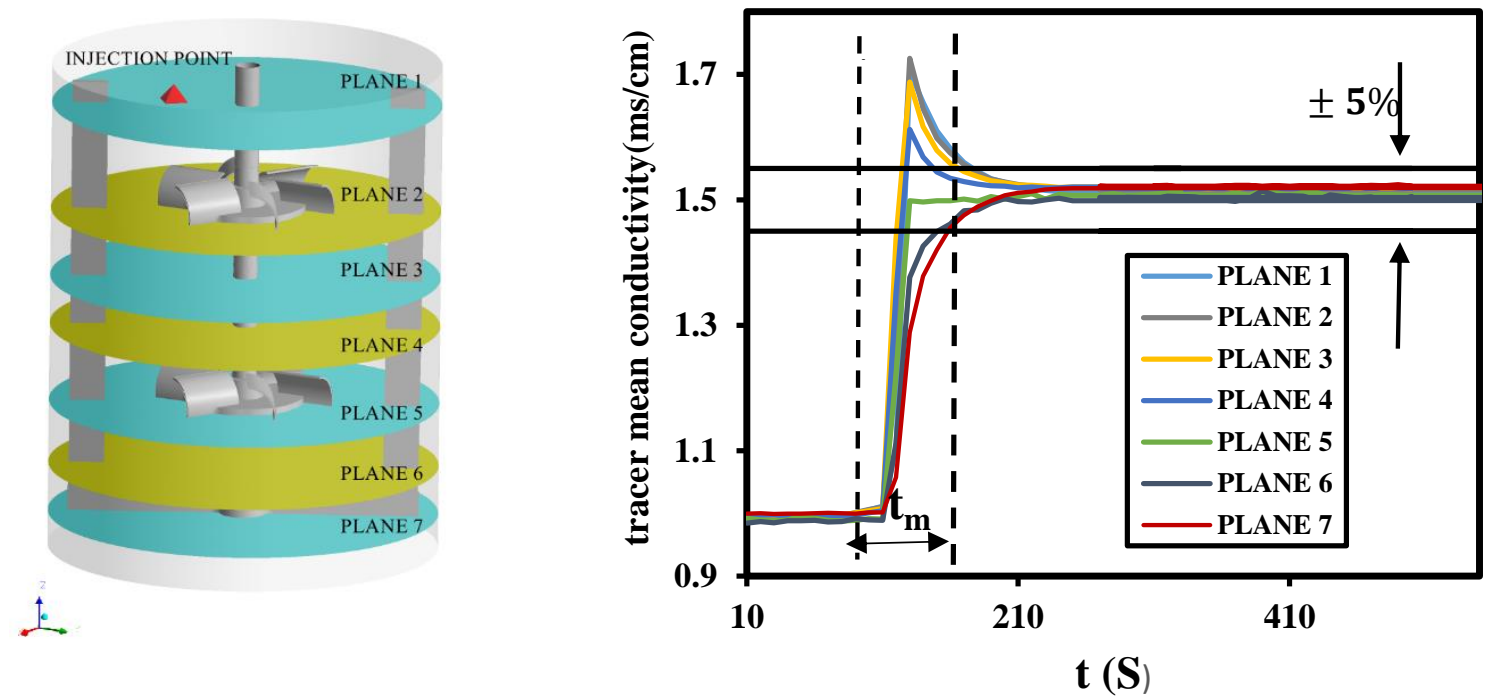

Figure (3.6.1). ERT planes and Mean conductivity measured using ERT as a function of time for $0.5 \%$ xanthan gum solution agitated by double Scaba-Anchor coaxial (DSAC) at $N_{a}=30 \mathrm{rpm}$ and $N_{s}=180 \mathrm{rpm}$.

Also electrical resistance tomography (ERT) as a non-intrusive visualization technique was utilized to visualize inside of the mixing tank, by monitoring the conductivity or concentration distribution of the tracer during the mixing process as depicted in Figure (3.6.2). 
(a)

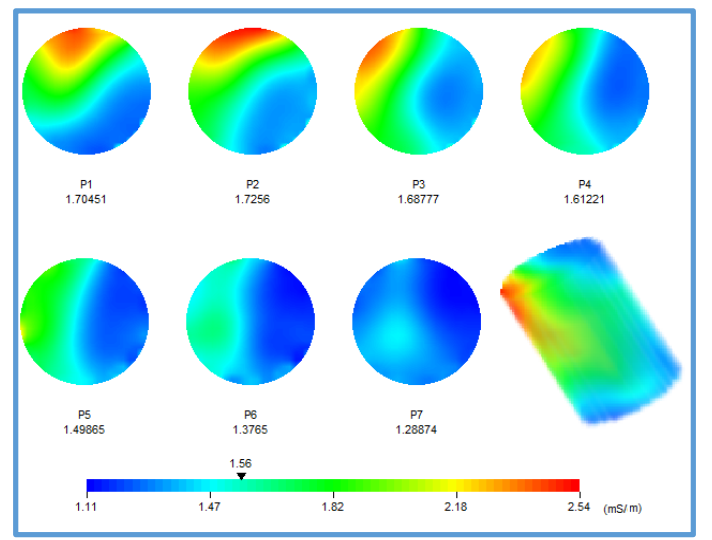

(b)

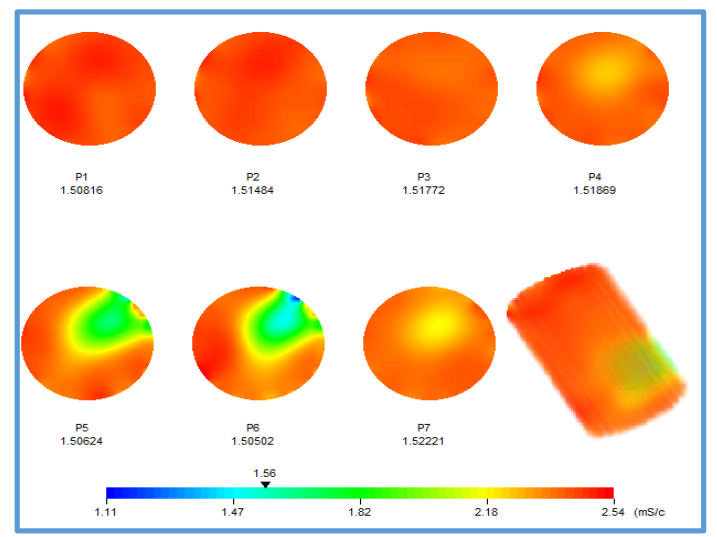

Figure (3.6.2). 2D and 3D tomography images at $R_{n}=8$, and $0.5 \%$ xanthan gum solution in the co-rotating mode for: (a) injection point at $t=100 \mathrm{~s}$, and (b) at $t=150 \mathrm{~s}$ by double Scaba-Anchor coaxial (DSAC) at $N_{a}=30 \mathrm{rpm}$ and $N_{s}=180 \mathrm{rpm}$.

\subsection{Error Analysis}

The errors regarding the torque sensors and ERT system have been analyzed as follows:

\subsubsection{Evaluation of Torque Sensor Precision}

The random error of the torque sensors (Models MCRT 48201V (2-2)-N-N and MCRT $48201 \mathrm{~V}$ (1-2)-N-N, S. Himmelstein and Company, USA) caused by electronic fluctuations, mechanical play, and friction can be defined using the standard deviation (SD):

$$
\mathrm{SD}=\sqrt{\frac{1}{N} \sum_{i=0}^{N}\left(X_{i}-\bar{X}\right)^{2}}
$$

where $\mathrm{N}$ is the number of measurements, and $\mathrm{X}$ is variable and $\bar{X}$ is the mean value of the measurements. It was found that the errors are small enough (SD $<0.20 \%$ ) to accept that the torque measurements were error independent. 


\subsubsection{Evaluation of ERT Measurements Precision}

As mentioned in Section 3.6, the ERT system was calibrated for each measurement. Each of the measurements was repeated three times and then the standard deviation was calculated. The insignificant standard deviations ( $\mathrm{SD}<0.65 \%$ ) were observed confirming a good repeatability and reproducibility of the experiments.

\subsection{Experimental Condition}

The effect of the central impeller type, impeller speed, impeller speed ratio, the rheological properties, and rotational mode on the agitation of the xanthan gum solutions with the different coaxial mixers were studied in terms of the power consumption, mixing time, flow characterization, and mixing energy. The experimental conditions for this research work are summarized in Table (3.8-1).

Table (3.8-1). Experimental conditions in this study.

\begin{tabular}{lc}
\hline \multicolumn{1}{c}{ Description } & Range and Type \\
\hline Central impeller types & Scaba, Rushton turbines, Pitched-blade \\
turbines with $45^{\circ}$
\end{tabular}




\section{Chapter 4}

\section{CFD Model}

CFD is the science of the predicting the three-dimensional fluid flow, heat and mass transfer, chemical reaction, and related phenomena. To predict these phenomena, the conservation equations of mass, momentum, and energy (called transport equations) are solved by the CFD software package through finite volume method via a coupled solver. In this study, the commercial CFD package called ANSYS FLUENT 15/Fluent (ANSYS ${ }^{\circ}$ Academic Research CFD, Release 15) was applied for the simulation of the flow domain generated by different configurations of coaxial mixers as shown in Figure (3.1-1). 


\subsection{Governing Equations}

In this study, conservation equations were solved for the simulation of the mixing of the incompressible xanthan gum solution with the coaxial mixers for an isothermal condition. To achieve this goal, the continuity and momentum equations were solved (Bird et al., 2002, Patankar, 1980; Ranade, 2002):

$$
\begin{aligned}
& \frac{\partial \rho}{\partial t}+\nabla \cdot(\rho \vec{v})=0 \\
& \frac{\partial}{\partial t}(\rho \vec{v})+\nabla \cdot(\rho \vec{v} \vec{v})=-\nabla p+\nabla \cdot(\bar{\tau})+\rho \vec{g}+\vec{F}
\end{aligned}
$$

where $\rho, \bar{\tau}, \rho \vec{g}, \vec{F}$ and $\vec{v}$ represent density, viscous stress tensor, gravitational force, external body force, and velocity.

A modified Herschel-Bulkley model was used to describe fluid rheology (Ford et al., 2006).

$$
\begin{array}{rlrl}
\mu & =\mu_{0} \text { for } \quad \tau \leq \tau_{y} & \\
\eta_{a} & =\frac{\left[\tau+K\left[\dot{\gamma}^{n}-\left(\frac{\tau_{y}}{\mu_{0}}\right)^{n}\right]\right.}{\dot{\gamma}} \text { for } \quad \tau>\tau_{y}
\end{array}
$$

Equations $[(4.1-3)$ and $(4.1-4)]$ show how shear stress $(\tau)$ varies with shear rate $(\dot{\gamma})$ for the Herschel-Bulkley fluids.

\subsection{Geometry}

The geometry of the agitated system was built in Design Modeler of ANSYS Fluent package software (Fluent 15). The system was divided to three parts, and then three zones 
were created: two identical rotating zones for two central impellers, and the rest was defined as anchor zone, which encapsulated the other zones. The impeller moving zone (i.e. Scaba) was a cylinder of $0.208 \mathrm{~m}$ diameter and $0.081 \mathrm{~m}$ height, and was centered on the impeller. The details of this geometry are shown in Figure (4.2-1). The geometry was then exported to the meshing part of Fluent.
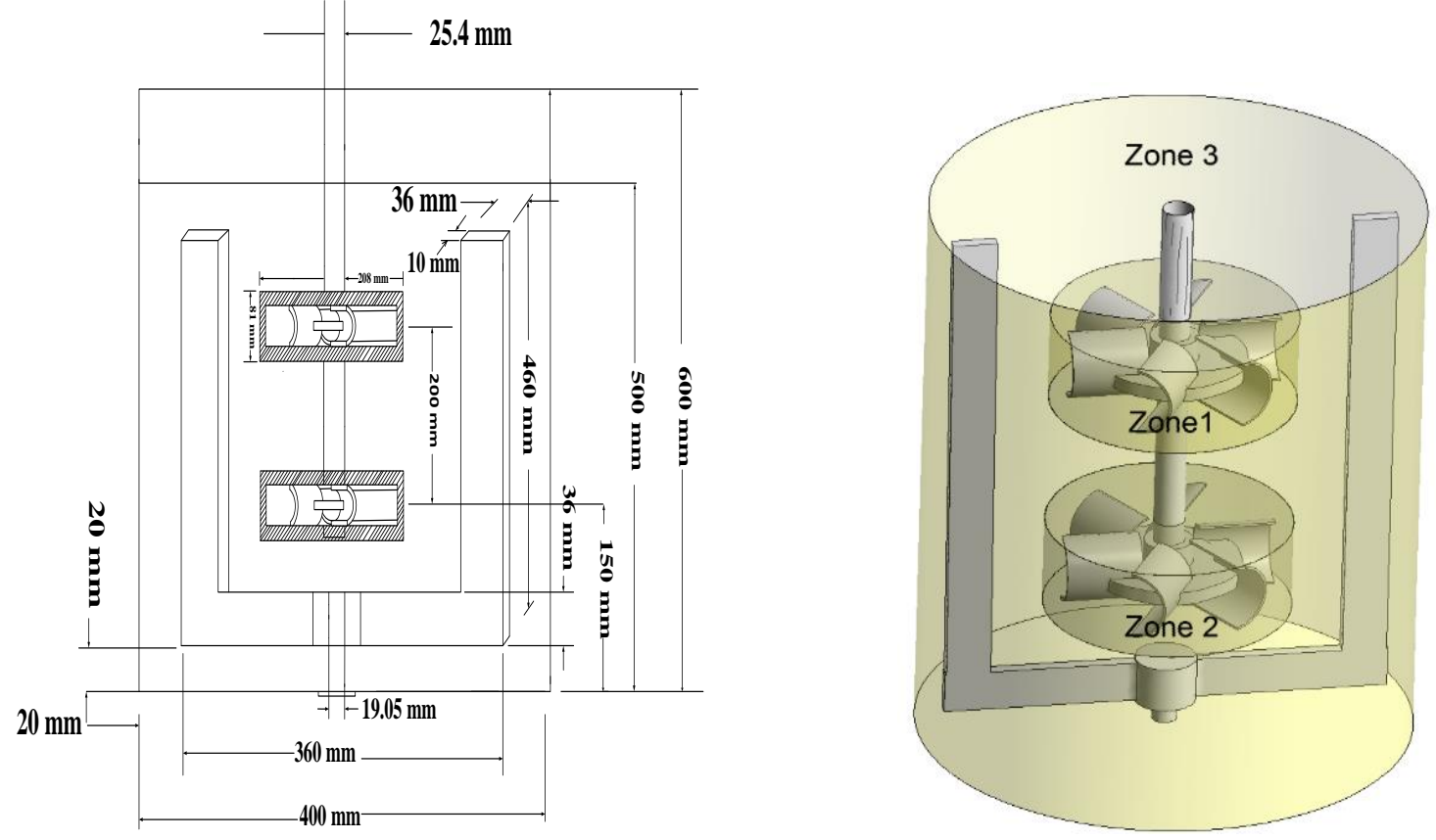

Figure 4.2-1 Geometry of developed model with specified zones.

\subsection{Grid Generation}

To solve the conservation equations of mass and momentum, the flow domain of the system was discretized into small volumes by means of the discretization grid. In this study, the 3D flow domain was mostly discretized with an unstructured tetrahedral grid. To capture the flow details, especially near the wall, smaller cells were generated near the 
solid walls to correctly solve the numerical equations. The mesh growth rate from the tank wall or the surface of the rotating impellers was controlled by the size function. Figure (4.3-1) illustrates the unstructured tetrahedral mesh for the coaxial mixing system.
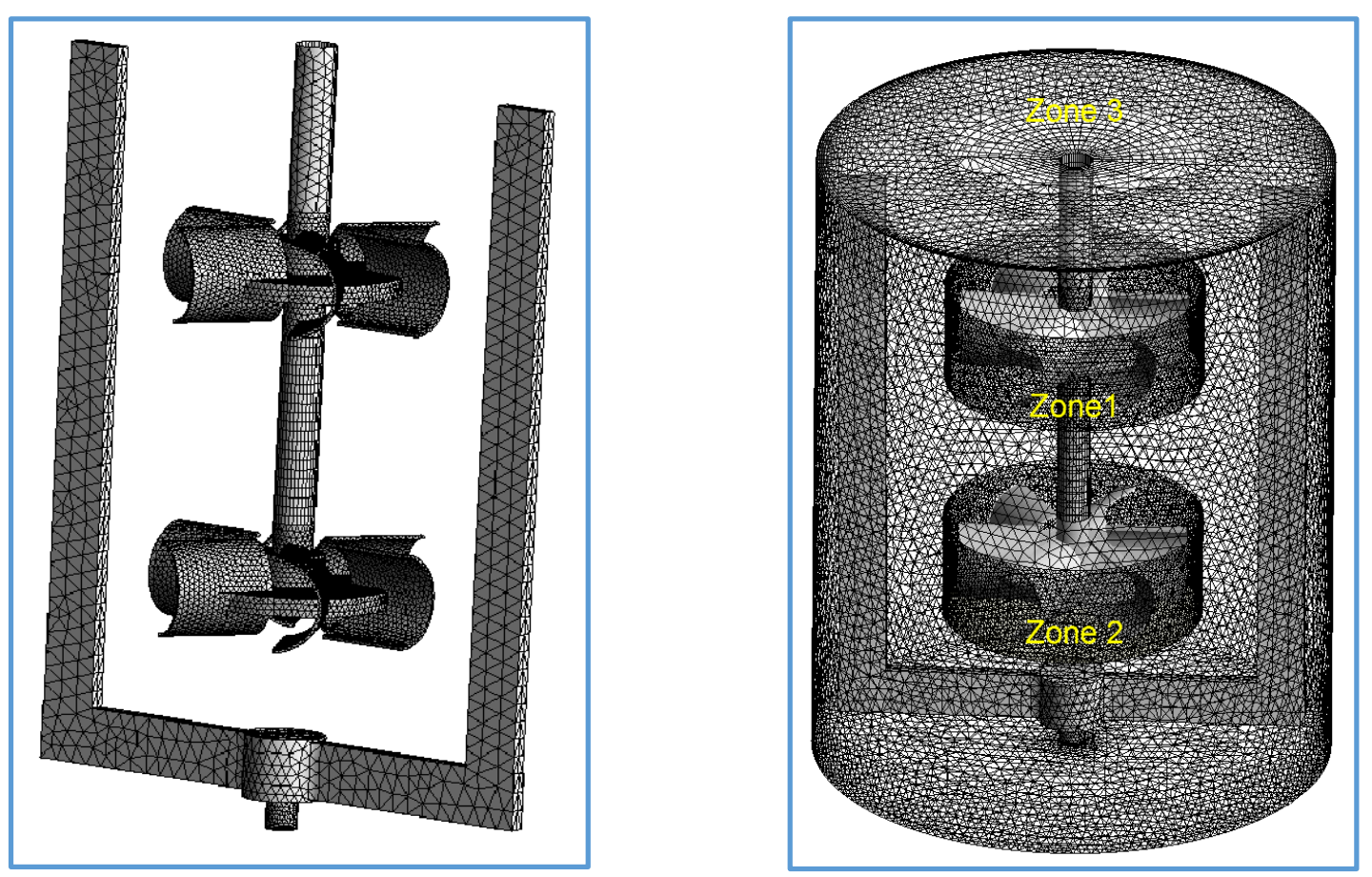

Figure (4.3-1). Unstructured tetrahedral mesh for coaxial system.

\subsubsection{Grid Independency}

The optimal number of grids was determined by conducting the grid independence test. Two approaches were utilized to study the grid independence: Maximum magnitude of velocity and velocity profile.

\subsubsection{Grid Independence Test through the Maximum Magnitude of Velocity}

The maximum magnitude of velocity in the vicinity of impeller was considered as the grid independence test parameter. In order to verify that the CFD results are grid 
independent, the number of cells increased by a factor of 2 . This approach has been employed by other researchers (Letellier et al., 2002; Buwa et al., 2006, Pakzad et al., 2013b) for the simulation of the agitated systems. In the first step, the number of grids was increased from 730,111(coarse) to 1,460, 222 (medium) cells. In the second step, the number of grids was increased from 1,460,222 (medium) to 2,920,444 (fine) cells. The result of these tests are shown in Table (4.1-1). According to these data, the optimal number of grids was $1,460,222$.

Table (4.3-1). Grid independence test results.

\begin{tabular}{c|l|c|c}
\hline Number of grids & $\mathbf{7 3 0 , 1 1 1}$ & $\mathbf{1 , 4 6 0 , 2 2 2}$ & $\mathbf{2 , 9 2 0 , 4 4 4}$ \\
\hline $\mathbf{V}^{*}{ }_{\text {Max }}(\mathbf{m} / \mathbf{s})$ & 0.9074 & 0.9394 & 0.9414 \\
\hline $\begin{array}{c}\text { Time required } \\
\text { (s) }\end{array}$ & 36,428 & 104,352 & 480,608 \\
\hline
\end{tabular}

\subsubsection{Grid Independence Study through Velocity Profile}

In the second approach, the radial, tangential, and axial velocity profiles in the regions of high-velocity gradients shown in Figure (4.3 -2) were computed for the coarse, medium, and fine grids. These results are presented in Figures [(4.3 - 3), (4-3 .4) and (4.3-5)]. 


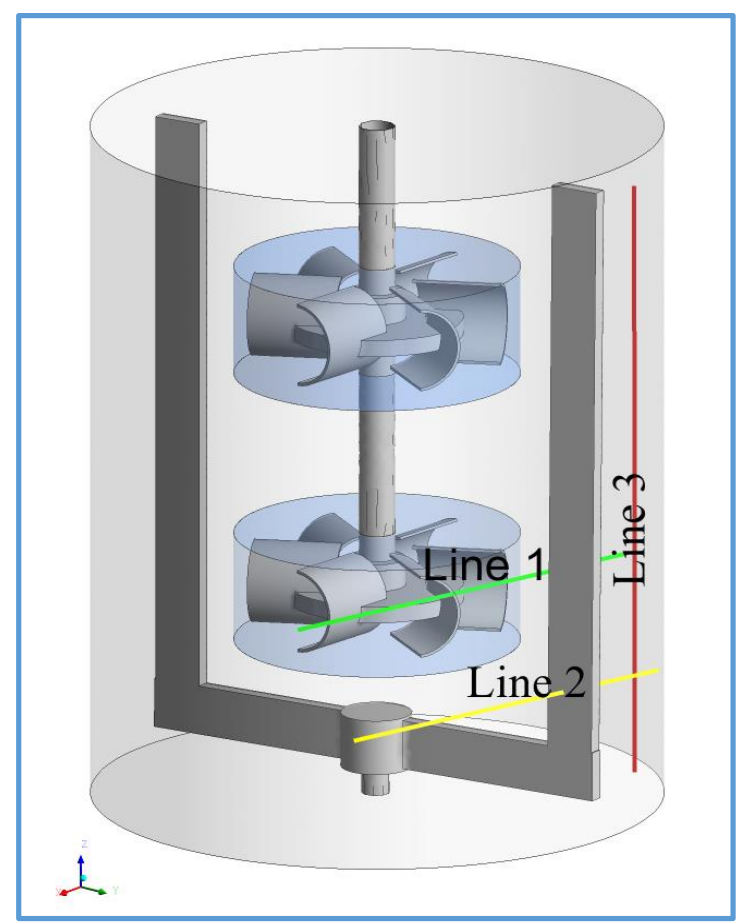

Figure (4.3-2). Selected lines for grid independence test.

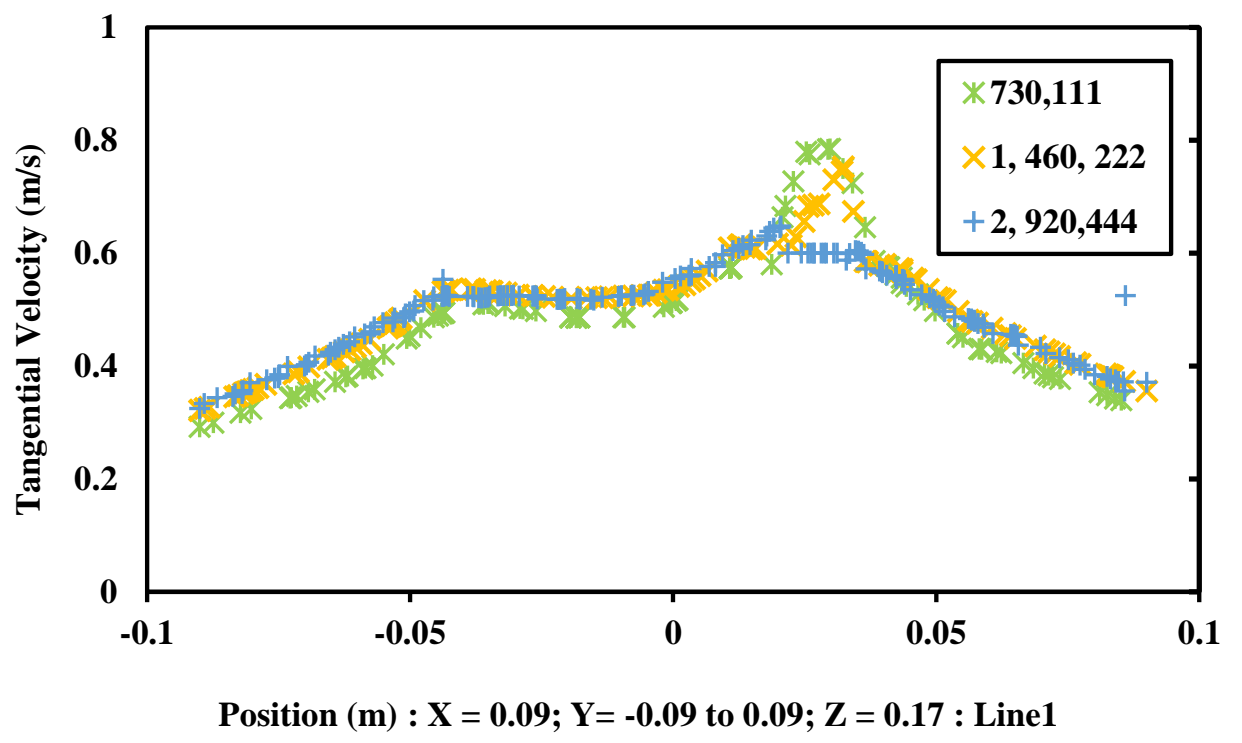

Figure (4.3-3). Effect of the number of grids on the tangential velocity in horizontal position close to the Scaba impeller [Line 1 in Figure (4.3-2)]. 
(a)

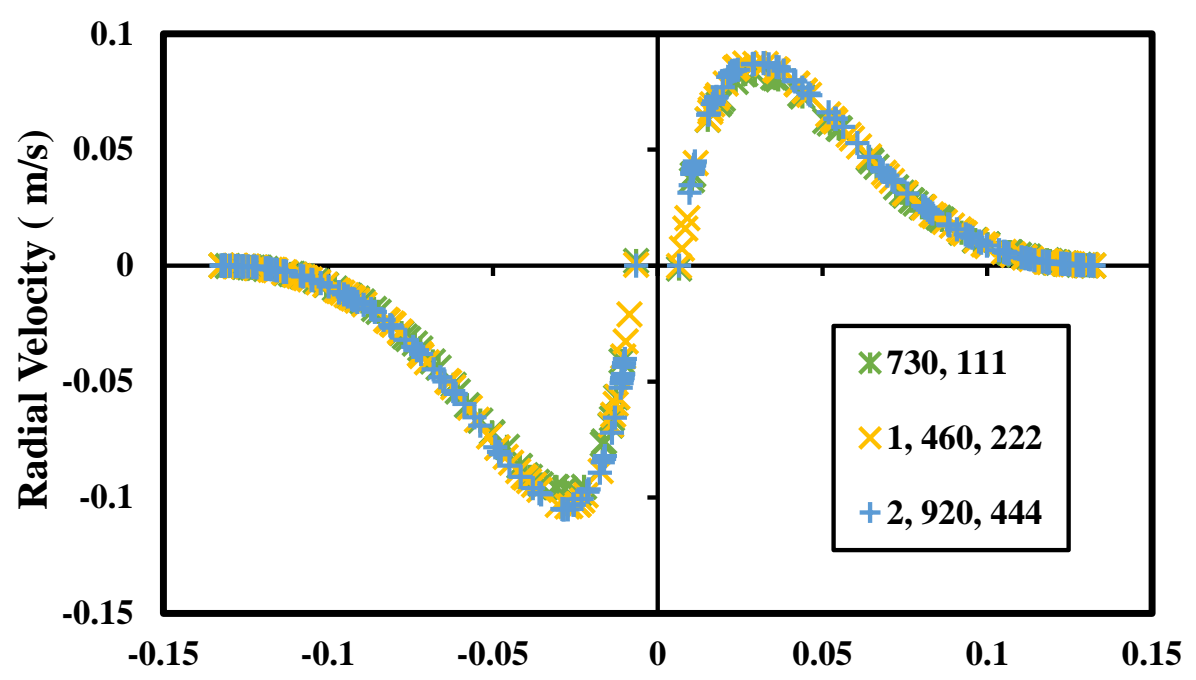

Position $(\mathrm{m}): \mathrm{x}=0.15 ; \mathrm{y}=-0.2$ to $0.2 ; \mathrm{z}=0.1$ : Line 2

(b)

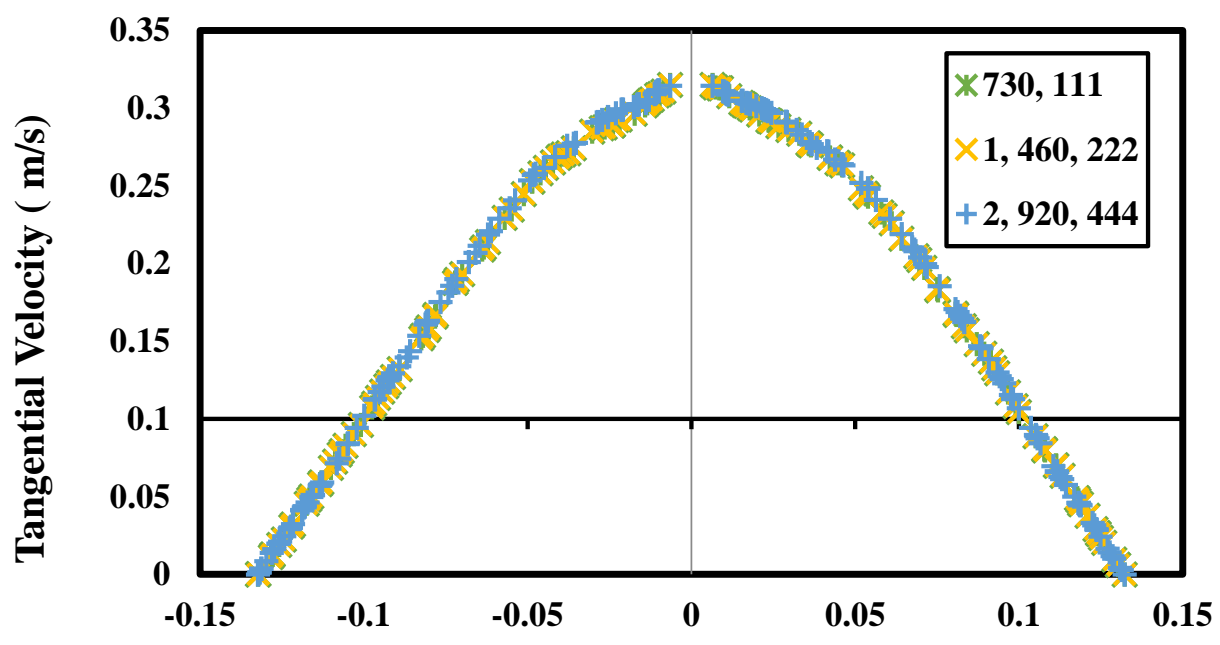

Position $(\mathrm{m}): \mathrm{x}=0.15 ; \mathrm{y}=\mathbf{- 0 . 2}$ to $0.2 ; \mathrm{z}=0.1$ Line 2

Figure (4.3-4). Effect of the number of grids on the (a) tangential velocity, and (b) Radial velocity in horizontal position close to anchor impeller [Line 2 in Figure (4.3-2)] 


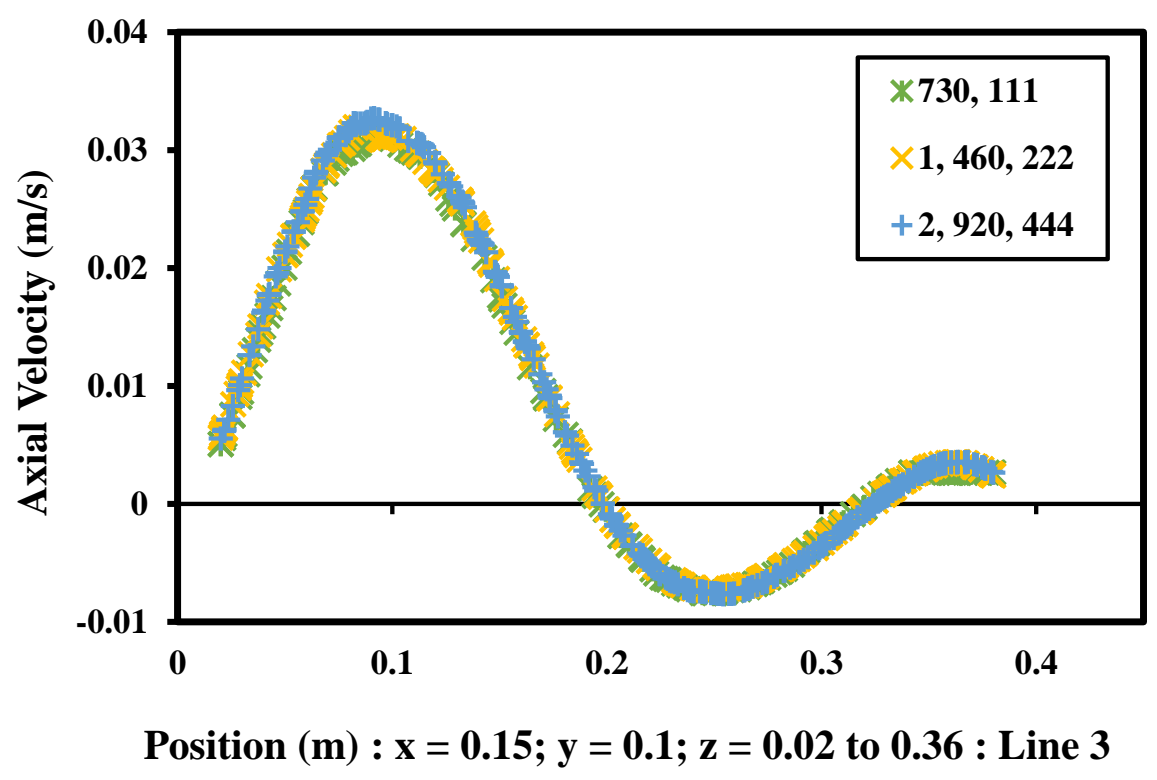

Figure (4.3-5). Effect of the number of grids on axial velocity in vertical situation close to the anchor impeller [Line 3 in Figure (4.3-2)].

These data show that when the number of cells was increased from 730,111 (coarse) to $1,460,222$ (medium), the velocity magnitude in the regions of high velocity gradients changed by more than $3.0 \%$. However, with increasing the number of grids from $1,460,222$ (medium) to $2,920,444$ (fine), the velocity magnitude in the regions of high velocity gradients was not changed considerably (less than 1.0\%). Thus, 1, 460,222 grids were applied for the simulation of the flow domain generated by the coaxial mixer in this work.

\subsection{Modeling of Stirred Tanks, Boundary Conditions and Solver Setting}

The multiple reference frame (moving reference frame) (Luo et al., 1994 and Tabor et al., 1996) for the steady state and the sliding mesh (moving or deforming domains) (Luo et al., 1993 and Lane and Koh, 1997) for unsteady state have been applied for the modeling 
of the impeller rotation inside the stirred vessels. These techniques have been developed to capture the motion of the rotating impeller in a stationary tank without requiring any empirical data. In this study, the sliding mesh method was employed for the development of the CFD model because of the interaction between the central and close clearance impellers in the coaxial mixing system. Due to the use of the sliding mesh for the simulations, three rotating grid zones were defined for the double central-anchor coaxial mixer: one around the upper central impeller (zone 1), one around the lower central impeller (zone 2), and one around the anchor impeller (zone 3) as shown in Figure (4.21). These zones can rotate independently with different rotational speeds and their sizes were kept constant for all numerical simulations. The central impeller zones and the anchor impeller zone were implicitly coupled by the interface separating the moving zones via a sliding-mesh where the required interpolations were performed due to the relative motion between the sub-domains.

Setting proper physical boundary conditions also supports the stability as well as the numerical convergence of CFD problem. Simulations were carried out using the laminar model based on the calculated Reynolds numbers. No-slip boundary condition $(V=0)$ was assumed on the tank wall, bottom and shaft. Zero normal velocity $\left(V_{z}=0\right)$ was assumed on the free surface of the fluid, and the tangential velocity was applied on the tip of each impeller (i: $\left.V_{\theta}=\pi N_{i} D\right)$ (Brucato et al., 1998). Second order up-wind for diffusion terms and second order interpolation for pressure were used. The SIMPLE algorithm (Patankar, 1980; Pakzad et al., 2013c) was used for velocity-pressure coupling. The selection of the time step is the most important step, which should be considered carefully because it has a significant impact on the convergence of the simulation results. 
A time step of $0.001 \mathrm{~s}$ was considered in all simulations. Usually, multiple reference frames can provide a stationary solution for the flow domain, so it was used as the initial condition for the moving mesh model. Applying such an initial condition could reduce the computational time to reach a quasi-steady state and periodic solution. In most numerical simulations, the periodic solution of the fluid flow domain was achieved after almost eight revolutions. The computational time for each simulation was about 36 hours. The convergence was achieved for each transport equation with the scale residuals below $10^{-7}$. The simulations were run in parallel with 12 dual cores SUN Ultra-Spark IV, $1.8 \mathrm{GHz}$ Sun Micro-Systems CPUs applying computing facilities of HPCVL (High Performance Computing Virtual Laboratory).

\subsection{Species Transport Model}

To estimate the mixing time, first the fully developed flow domain generated in the mixing vessel was simulated. Then the second fluid (called tracer) was injected under the liquid surface. The dynamic distribution of the tracer inside of agitated system was computed using the following time dependent scalar species transport equation.

$$
\frac{\partial}{\partial t}(\rho w)+\nabla \cdot(\rho \bar{v} w)=\nabla \cdot \rho D_{m} \nabla w
$$

Here, $w$ is the local mass fraction of the tracer, $\bar{v}$ is the mean velocity vector, $\rho$ is the fluid density, $D_{m}$ is the molecular diffusivity of the tracer in the mixture. It is assumed that the distribution of the tracer takes place based on convection and diffusion.

To apply the species transport model for mixing time, the simulation of the fluid flow was performed first. Then, the equations of flow field were deactivated. Following that, 
the mass fraction of tracer was set to 1 for injected region and the concentration of tracer in the rest of the vessel volume was patched to zero at time $t=0$. The concentration of tracer was then monitored at the same locations as the ERT planes (Figure 3.6-1). The influence of the time step on the tracer concentration profiles was checked by performing the simulations for the time steps of $0.01,0.1$ and $1 \mathrm{~s}$. The computed mixing times $\left(t_{95}\right)$ at time steps of 1 and $0.1 \mathrm{~s}$ were different. However, the same mixing time values were attained for the time steps of 0.01 and $0.1 \mathrm{~s}$ with the computational times of 28 and 6-7 hours, respectively. Therefore, the time step of $0.1 s$ was applied for the mixing time simulations in this study and the number of time step was set to 700 . The scale residual for species transport equation was set under $10^{-5}$. The molecular diffusivity of the tracer in the mixture was assumed to be $10^{-9}\left(\mathrm{~m}^{2} \mathrm{~s}^{-1}\right)$ as a typical value for liquids (Montante et al. 2005; Pakzad et al., 2013a). When the concentration of the tracer at defined monitoring points reached to $95 \%$ of the steady value, the time was considered as the mixing time. The results are shown in Figure (4.5-1). 

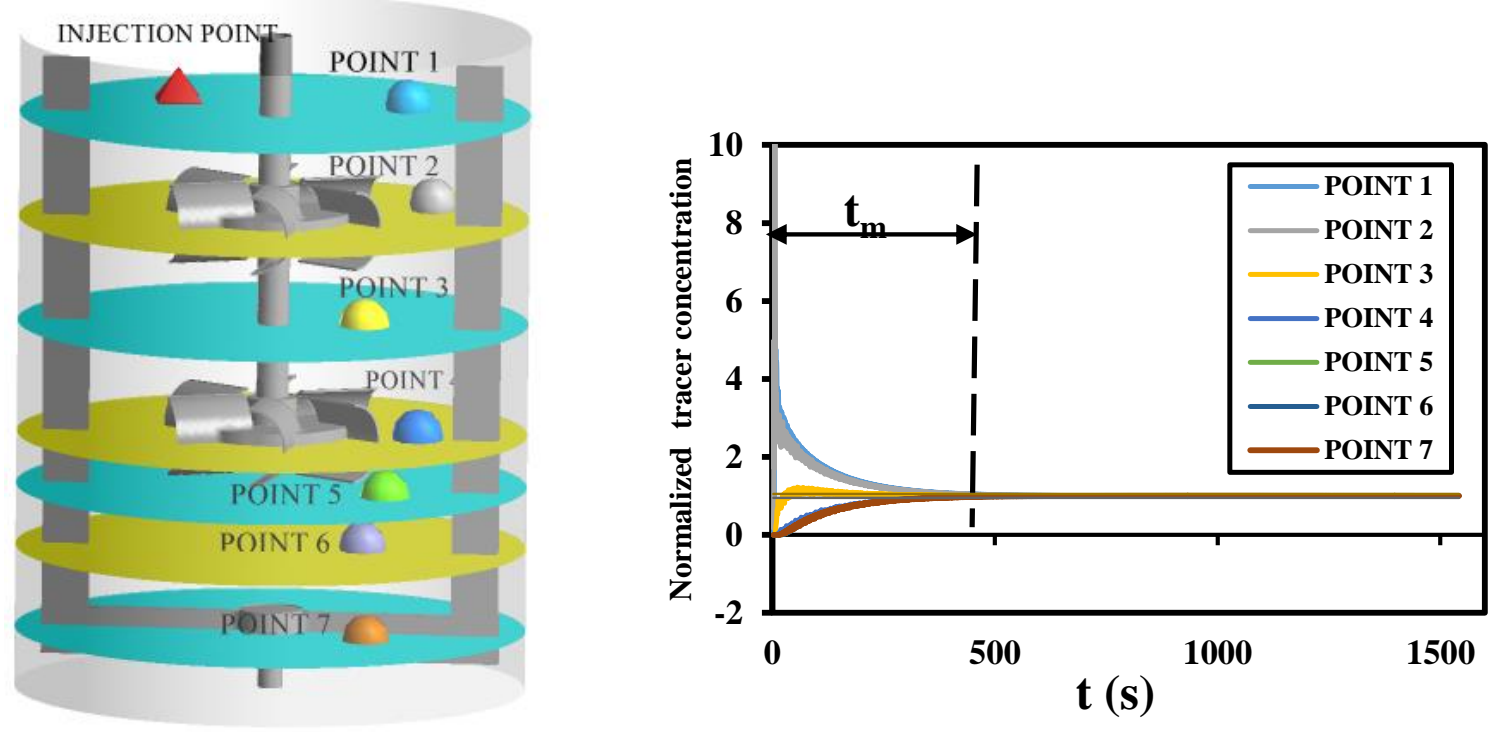

Figure (4.5-1). Monitoring planes and normalized tracer concentration using simulated CFD model as a function of time for $1.0 \%$ xanthan gum solution agitated by the ScabaScaba-Anchor coaxial (SSAC) at $N_{a}=30 \mathrm{rpm}$ and $N_{s}=180 \mathrm{rpm}$.

To produce further information, the CFD models were validated by comparing the experimentally determined mixing time and torque measurements with computationally determined mixing time and torque measurements for different concentration at different speed ratios. A good agreement (maximum relative error $<5 \%$ ) was achieved between the computed torque and mixing times and experimentally obtained values as shown in Table (4.5-2). This approach was adopted for all developed models in this study and the validated models were then utilized to obtain further information for the assessment of the investigated coaxial mixers in this study. 
Table (4.5-2) Computed and measured torque and mixing time for $0.5 \%$ xanthan gum solution agitated at $R_{n}=8$ by the Scaba-anchor coaxial mixer.

\begin{tabular}{|c|c|c|c|c|c|c|c|}
\hline & & $\begin{array}{c}\text { Measured } \\
\text { Torque }\end{array}$ & $\begin{array}{c}\text { Computed } \\
\text { Torque }\end{array}$ & $\begin{array}{c}\text { Relative } \\
\text { Error }\end{array}$ & $\begin{array}{c}\text { Measured } \\
\text { Mixing } \\
\text { Time } \\
\end{array}$ & $\begin{array}{c}\text { Computed } \\
\text { Mixing } \\
\text { Time } \\
\end{array}$ & $\begin{array}{c}\text { Relative } \\
\text { Error }\end{array}$ \\
\hline No & $\begin{array}{l}\text { Scaba- } \\
\text { Anchor }\end{array}$ & (N.m) & (N.m) & $(\%)$ & $(\min )$ & $(\min )$ & $(\%)$ \\
\hline \multirow[b]{2}{*}{1} & Scaba & 0.317 & 0.328 & 3.4 & \multirow[b]{2}{*}{8.31} & \multirow[b]{2}{*}{8.74} & \multirow[b]{2}{*}{4.9} \\
\hline & Anchor & & 0.426 & 3.3 & & & \\
\hline \multirow[b]{2}{*}{2} & Scaba & 0.462 & 0.483 & 4.4 & \multirow[b]{2}{*}{7.36} & \multirow[b]{2}{*}{7.65} & \multirow[b]{2}{*}{3.8} \\
\hline & Anchor & 0.349 & 0.365 & 4.4 & & & \\
\hline \multirow{2}{*}{3} & Scaba & 0.579 & 0.607 & 4.6 & \multirow{2}{*}{6.82} & \multirow{2}{*}{7.16} & \multirow{2}{*}{4.7} \\
\hline & Anchor & 0.284 & 0.298 & 4.7 & & & \\
\hline \multirow[b]{2}{*}{4} & Scaba & 0.659 & 0.684 & 3.7 & \multirow[b]{2}{*}{5.66} & \multirow[b]{2}{*}{5.94} & \multirow[b]{2}{*}{4.8} \\
\hline & Anchor & 0.237 & 0.246 & 3.7 & & & \\
\hline \multirow{2}{*}{5} & Scaba & 0.760 & 0.798 & 4.8 & \multirow{2}{*}{5.29} & \multirow{2}{*}{5.31} & \multirow{2}{*}{3.8} \\
\hline & Anchor & 0.174 & 0.183 & 4.8 & & & \\
\hline
\end{tabular}




\section{Chapter 5}

\section{Results and Discussion}

In this study, the performance of the coaxial mixers composed of two central impellers and one anchor in the agitation of complex fluids was investigated. To fulfill this objective, computational fluid dynamics (CFD), electrical resistance tomography (ERT), and design of experiments (DOE) combined with response surface methodology (RSM) were employed. The hydrodynamics performances of coaxial mixers were evaluated in term of power consumption, mixing time, flow characterization, pumping effectiveness, mixing efficiency, and mixing energy. In this chapter all results and related discussions are presented in the following five sections: 
- Effect of the Rheological Properties on the Mixing of Herschel-Bulkley Fluids with the Coaxial Mixers: Applications of Tomography, CFD, and Response Surface Methodology

Section 5.1 mainly deals with the effect of the rheological properties of the yieldpseudoplastic fluids (consistency index $(K)$, power-law index $(n)$, and yield stress $\left(\tau_{y}\right)$ ) on the hydrodynamic performance of the Scaba-anchor coaxial mixer. To achieve this objective, the performance of the Scaba-anchor coaxial mixer in the mixing of xanthan gum solutions (yield-pseudoplastic fluids) was evaluated through ERT, CFD, and DOE combined with RSM data. The results presented in this section have already been published for publication in the Canadian Journal of Chemical Engineering (Kazemzadeh et al., 2016a).

- Investigation on Hydrodynamic Performances of Coaxial Mixers in Agitation of Yield-Pseudoplasitc Fluids: Single and Double Central Impellers in Combination with the Anchor

In section 5.2, we compare the hydrodynamic performances of two coaxial mixers consisting of the single and double Scaba impellers in combination with the anchor in the agitation of the xanthan gum solution (non-Newtonian yield-pseudoplastic fluids) in the laminar-transitional regime, both numerically and experimentally. The 3D numerical simulations of flow domain produced by these two types of the coaxial mixers was made by the CFD. ERT was used to visualize inside of the mixing tank and to measure mixing time. In this work, the performances of the coaxial mixers were evaluated in terms of mixing time, fluid characterization, and power consumption. The results discussed in this 
section have already been published in Chemical Engineering Journal (Kazemzadeh et al., 2016b).

\section{- A New Perspective in the Evaluation of the Mixing of Biopolymer Solutions with Different Coaxial Mixers Comprising of Two Dispersing Impellers and a Wall Scraping Anchor}

Section 5.3 provides a discussion on the hydrodynamic performances of coaxial mixers consisting of a close clearance anchor impeller and dual central impellers in the agitation of the xanthan gum solution, a yield-pseudoplastic biopolymer solution, in the laminartransitional regimes in the co-rotating mode. The different coaxial mixers utilized in this study were dual Scaba impellers, dual Rushton turbines, and dual pitched blade turbines in combination with an anchor impeller. Considering both numerical and experimental approaches, three techniques were employed: computational fluid dynamics (CFD) to simulate the flow domain of the fluid, electrical resistance tomography (ERT) to measure mixing time and visualize flow behavior inside the stirred vessel, and design of experiments (DOE) combined with the response surface methodology (RSM) to analyze the data. The experimental and numerical data were collected to assess the performances of the coaxial mixers in the mixing of the complex fluids with respect to the power consumption, mixing time, velocity vector plots, streamline plots, axial, radial and tangential velocity profiles, axial shear strain rate profiles, and flow number. The results presented in this section have already been accepted by Chemical Engineering Research and Design (Kazemzadeh et al., 2016c). 
- An Investigation on the Effect of the Impeller Spacing on the Flow Field Generated by the Coaxial Mixing System Composed of Double Central Impellers and an Anchor in the Agitation of Yield-Pseudoplastic Fluids

In Section 5.4, In order to evaluate the influence of the central impeller spacing on the hydrodynamic performance of the double Scaba-anchor coaxial mixer, the upper impeller submergence was set to $0.140 \mathrm{~m}$ while the lower impeller clearance and the spacing between two central impellers were changed over a wide range. Furthermore, the impact of the impeller spacing in the co-rotating mode was assessed with respect to the mixing time, power number, and mixing energy. The results presented in this section have already been submitted to Chemical Engineering Communications (Kazemzadeh et al., 2016d).

\section{- Mixing of non-Newtonian Biopolymer Solutions with the Coaxial Mixers Composed of Two different Central Impellers and an Anchor}

In Section 5.5, the performances of different configurations of coaxial mixers composed of a wall scraping anchor impeller in combination with two different or identical central high speed impellers in the agitation of the yield-pseudoplastic xanthan gum solution were investigated. The coaxial mixers utilized in this study were the Scaba-Scaba-anchor, Scaba-Rushton-anchor, Rushton-Scaba- anchor, Scaba-pitched blade-anchor, and pitched blade-Scaba-anchor. A new correlation was introduced for these complex configurations of the coaxial mixers by incorporating the Metzner-Otto constants $\left(K_{s}\right)$ of the different types of the central impellers into the Reynolds number. The experimental and CFD data were employed to evaluate the performances of these five coaxial mixers with respect to the power consumption, mixing time, velocity profiles, shear strain rate profiles, flow 
number, power number, and pumping effectiveness. The results presented in this section have already been submitted to Chemical Engineering and processing (Kazemzadeh et al., 2016e). 


\subsection{Effect of the Rheological Properties on the Mixing of Herschel- Bulkley Fluids with the Coaxial Mixers: Applications of Tomography, CFD, and Response Surface Methodology}

\subsubsection{Introduction}

The unique objective of this study was to explore the influence of the rheological properties of the yield-pseudoplastic fluids (consistency index $(K)$, power-law index $(n)$, and yield stress $\left(\tau_{y}\right)$ ) on the hydrodynamic performance of the Scaba-anchor coaxial mixer [Figure 5.1-1)] using electrical resistance tomography (ERT) and computational fluid dynamics (CFD). Design of experiments (DOE) and response surface methodology (RSM) were used to determine the number of tests, to analyze the data, and to determine the optimal design parameters. The developed CFD model was validated by the experimentally measured power drawn and mixing time [Table (5.1.1)]. The validated CFD model was then used to estimate the influence of the rheological properties of fluid on the mixing efficiency at different speed ratios.
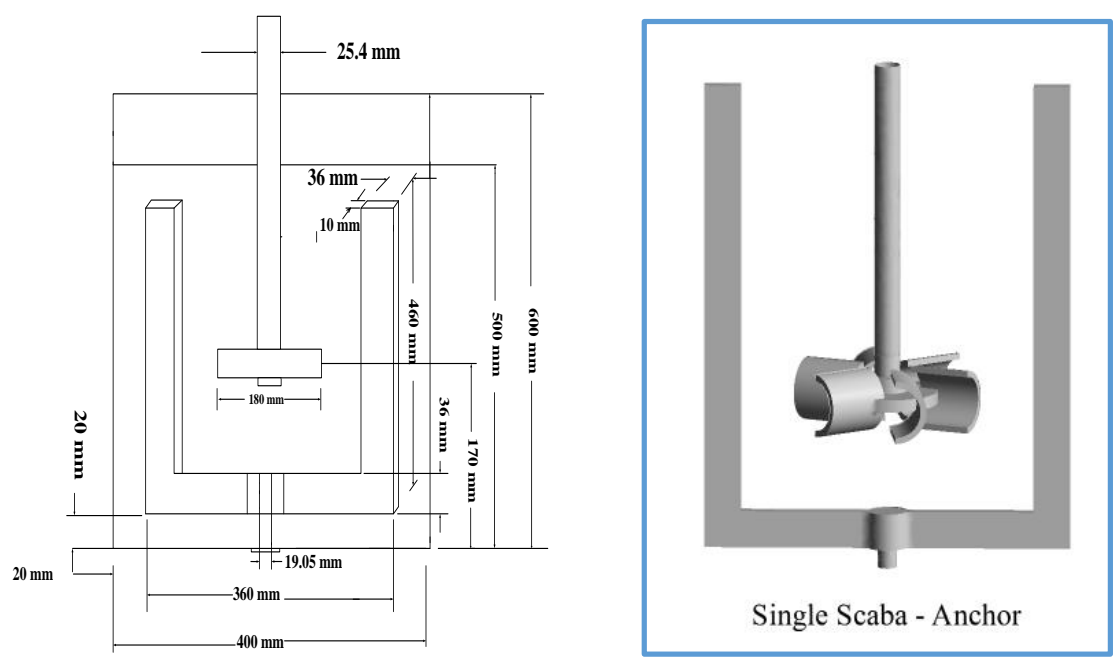

Figure (5.1-1). The configuration of the coaxial mixers used in this study. 
Table (5.1-1). Computed and measured torque and mixing time for $1.0 \%$ xanthan gum solution agitated by the Scaba-anchor coaxial mixer.

\begin{tabular}{|c|c|c|c|c|c|c|c|}
\hline & & $\begin{array}{c}\text { Measured } \\
\text { Torque }\end{array}$ & $\begin{array}{c}\text { Computed } \\
\text { Torque }\end{array}$ & $\begin{array}{c}\text { Relative } \\
\text { Error }\end{array}$ & $\begin{array}{c}\text { Measure } \\
\text { d } \\
\text { Mixing } \\
\text { Time }\end{array}$ & $\begin{array}{l}\text { Computed } \\
\text { Mixing } \\
\text { Time }\end{array}$ & $\begin{array}{c}\text { Relative } \\
\text { Error }\end{array}$ \\
\hline $\begin{array}{l}\text { Speed } \\
\text { Ratio }\end{array}$ & & (N.m) & (N.m) & $(\%)$ & (s) & (s) & $(\%)$ \\
\hline$R_{n}=1.7$ & $\begin{array}{l}\text { Scaba } \\
\text { Anchor }\end{array}$ & $\begin{array}{l}0.175 \\
0.622\end{array}$ & $\begin{array}{l}0.185 \\
0.646\end{array}$ & 3.8 & 115.0 & 121 & 4.9 \\
\hline$R_{n}=3.0$ & $\begin{array}{l}\text { Scaba } \\
\text { Anchor }\end{array}$ & 0.492 & 0.510 & 3.5 & 91.2 & 95 & 4.0 \\
\hline$R_{n}=4.0$ & Anchor & $\begin{array}{l}0.551 \\
0.451\end{array}$ & $\begin{array}{l}0.575 \\
0.470\end{array}$ & 4.0 & 81.5 & 85 & 4.1 \\
\hline$R_{n}=5.0$ & $\begin{array}{l}\text { Scaba } \\
\text { Anchor }\end{array}$ & $\begin{array}{l}0.874 \\
0.308\end{array}$ & $\begin{array}{l}0.905 \\
0.320\end{array}$ & $\begin{array}{l}3.4 \\
3.8\end{array}$ & 74.5 & 78 & 4.4 \\
\hline$R_{n}=6.0$ & $\begin{array}{l}\text { Scaba } \\
\text { Anchor }\end{array}$ & 0.062 & $\begin{array}{l}1.300 \\
0.064 \\
\end{array}$ & 3.8 & 67.9 & 71 & 4.3 \\
\hline
\end{tabular}

The effect of the rheological parameters of the Herschel-Bulkley fluid on the mixing efficiency of the coaxial mixing system at different speed ratios was obtained using BoxWilson model (Box et al., 1951) known as the central composite design (CCD) combined with the response surface methodology (RSM) (Ray et al., 2009). In this study, the proposed experimental range and the level of each independent variable (consistency index, power-law index, yield stress, and speed ratio of the impellers) are listed in Table (5.1-2). The data from the CCD method was fitted into the following quadratic function, which was a second order equation: 


$$
Y=B_{0}+\sum_{i=1}^{k} B_{i} X_{i}+\sum_{i=1}^{j-1} \sum_{j=1}^{k} \beta_{i j} X_{i} X_{j}+\sum_{i=1}^{k} \beta_{i i} X_{i}^{2}+e_{i}
$$

where $Y, X_{i} X_{j}, \beta_{0}, \beta_{i}, \beta_{i j}, \beta_{i i}, \mathrm{k}$, and $e_{i}$ represent: predicted response, independent variables, constant coefficient, the influence of independent variable, the influence of interaction among variables, quadratic effect, the number of variables, and error residual.

Table (5.1-2). Experimental range and levels of the independent variables.

\begin{tabular}{lcccccc}
\hline Variables & Symbol & -2 & -1 & 0 & 1 & 2 \\
\hline Power-law index (-) & $X_{1}$ & 0.12 & 0.2 & 0.4 & 0.6 & 0.8 \\
Consistency index $\left(\mathrm{Pa} \mathrm{s}^{n}\right)$ & $X_{2}$ & 3 & 8 & 14 & 19 & 28 \\
Yield stress (Pa) & $X_{3}$ & 1.78 & 5.25 & 11.80 & 14.30 & 20.60 \\
Speed ratio (-) & $X_{4}$ & 1.7 & 3.0 & 4.0 & 5.0 & 6.0 \\
\hline
\end{tabular}

\subsubsection{Results and Discussion}

The mixing time $t_{m}$ and power drawn $P$ response functions based on Equation (5.1.1) were obtained as quadratic equations by applying multiple regression analysis:

$$
\begin{aligned}
t_{m} & =180+52.21 K+42.21 \tau_{y}-16.46 R_{n}-2.81 K \tau_{y^{-}} 6.22 R_{n}{ }^{2} \\
P & =23.42-1.85 n+9.44 K+2.34 \tau_{y}+15.05 R_{n}-1.19 n K-1.45 n R_{n} \\
& +1.26 K \tau_{y}+4.72 K R_{n}-1.22 \tau_{y}{ }^{2}+3.06 R_{n}{ }^{2}
\end{aligned}
$$

The significance of the fit of the quadratic functions for experimental data were examined using the analysis of variance (ANOVA). The coefficient determination $\left(R^{2}=0.9775\right.$ for mixing time and $R^{2}=0.9840$ for power consumption) confirmed the goodness of fit of the models. It means that only $2.25 \%$ of variability for mixing time and $1.60 \%$ for power 
consumption were not explained by the response equations. The outcome of ANOVA for both response functions of $t_{m}$ and $P$ are summarized in Tables (5.1-3) and (5.1-4), respectively. If the values of Prob > F listed in the last column on the right side of these two tables are less than 0.0500 , the model is considered to be significant. When these values are greater than 0.1000 , the model becomes insignificant. For instance, the ANOVA results tabulated in (Table 5.1-3) shows that the power-law index, $n$, did not have a significant effect on the mixing time (Prob $>0.1$ ). Thus, it was excluded from the mixing time response function.

Table (5.1-3). ANOVA test for response function (mixing time) $t_{m}=180+52.21 \mathrm{~K}+$ $42.21 \tau_{y}-16.46 R_{n}-2.81 K \tau_{y^{-}}-6.22 R_{n}^{2}$.

\begin{tabular}{cccccc}
\hline Source & $\begin{array}{c}\text { Sum of } \\
\text { Squares }\end{array}$ & $\begin{array}{c}\text { Degree of } \\
\text { freedom }\end{array}$ & Mean Square & F-ratio & $\begin{array}{c}P \text {-value } \\
\text { Prob }>\text { F }\end{array}$ \\
\hline Model & $1.30 \times 10^{5}$ & 14 & 8989.03 & 10.51 & $<0.0001$ \\
$n$ & 1488.38 & 1 & 1488.38 & 1.74 & 0.2069 \\
$K$ & 65417.04 & 1 & 65417.02 & 76.74 & $<0.0001$ \\
$\tau_{y}$ & 42757.04 & 1 & 45757.04 & 49.98 & $<0.0001$ \\
$R_{n}$ & 6501.04 & 1 & 6501.04 & 7.60 & $<0.0147$ \\
$K \tau_{\mathrm{y}}$ & 5513.06 & 1 & 5513.06 & 6.44 & 0.0227 \\
$\tau_{y}{ }^{2}$ & 1060.74 & 14 & 1060.74 & 1.24 & 0.2830 \\
$R_{n}{ }^{2}$ & 2524.53 & 1 & 2524.53 & 2.95 & 0.1000 \\
\hline
\end{tabular}


Table (5.1-4). ANOVA test for response function (power consumption) $P=23.42-1.85 n$

\begin{tabular}{cccccc}
$+9.44 K+2.34 \tau_{y}+15.05 R_{n}-1.19 n K-1.45 n R_{n}+1.26 K \tau_{y}+4.72 K R_{n}-1.22 \tau_{y}{ }^{2}+3.06 R_{n}{ }^{2}$. \\
\hline Source & Sum of & Degree of & Mean & F-ratio & P-value \\
& Square & & Prob $>\mathrm{F}$ \\
\hline Model & 8561.54 & 10 & 856.15 & 116.15 & $<0.0001$ \\
$n$ & 82.14 & 1 & 82.14 & 11.19 & $<0.0034$ \\
$K$ & 2136.99 & 1 & 2136.99 & 291.04 & $<0.0001$ \\
$\tau_{\mathrm{y}}$ & 131.41 & 1 & 131.41 & 17.90 & 0.0005 \\
$R_{\mathrm{n}}$ & 5437.87 & 1 & 5437.87 & 740.59 & $<0.0001$ \\
$n K$ & 22.52 & 1 & 22.52 & 3.07 & 0.0961 \\
$n R_{n}$ & 33.58 & 1 & 33.58 & 4.57 & $<0.0457$ \\
$K \tau_{y}$ & 25.55 & 1 & 25.55 & 3.48 & 0.0776 \\
$K R_{n}$ & 355.89 & 1 & 355.89 & 48.47 & $<0.0001$ \\
$\tau_{3 y}{ }^{2}$ & 24.11 & 1 & 42.11 & 5.74 & $<0.0271$ \\
$R_{n}{ }^{2}$ & 265.82 & 1 & 265.82 & 36.20 & $<0.0001$ \\
\hline
\end{tabular}

Using ANOVA results also enabled us to examine the interaction among variables affecting the response functions of mixing time and power consumption. Table (5.1-3) demonstrates that the interaction between power-law index and consistency index $(n K)$, power-law index and yield stress $\left(n \tau_{y}\right)$, power-law index and speed ratio $\left(n R_{n}\right)$, consistency index and speed ratio $\left(K R_{n}\right)$, and yield stress and speed ratio $\left(\tau_{y} R_{n}\right)$ were insignificant for the mixing time response because of Prob $>0.1$. As a consequence, the interactions of $n K, K \tau_{y}, n R_{n}, K R_{n}, \tau_{y} R_{n}$ were ruled out from the response function of the mixing time. The interaction between consistency index and yield stress $\left(K \tau_{y}\right)$ was the only interaction considered in the mixing time model [Equation (5.1-2)]. Table (5.14) shows that the interaction between power-law index and yield stress $\left(n \tau_{y}\right)$, and between yield stress and speed ratio $\left(\tau_{y} R_{n}\right)$ were insignificant for power consumption response due to Prob $>0.1$. Thus, these interactions were removed and the other 
interactions $\left(n K, K R_{n}, K \tau_{y}\right.$, and $\left.K R_{n}\right)$, which were significant, were included in the power consumption response [Equation (5.1-3)]. It is worth mentioning that the interaction between consistency index and yield stress $\left(K \tau_{y}\right)$ is significant for both responses. Figure (5.1-2) shows a close agreement between the values predicted by Equation (5.1-2) and Equation (5.1-3) and the experimental data for the ranges of the variables investigated in this study.

(a)

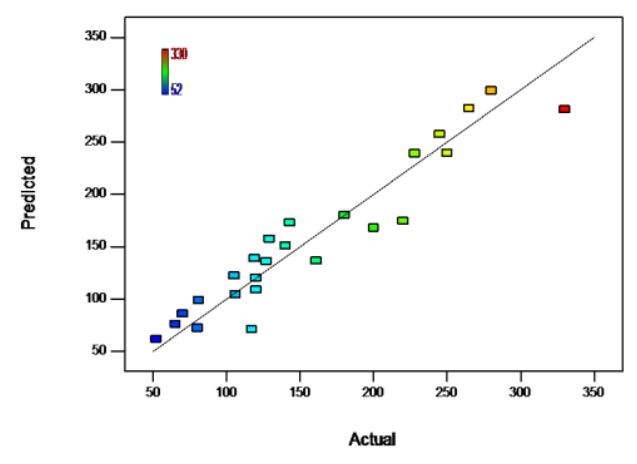

(b)

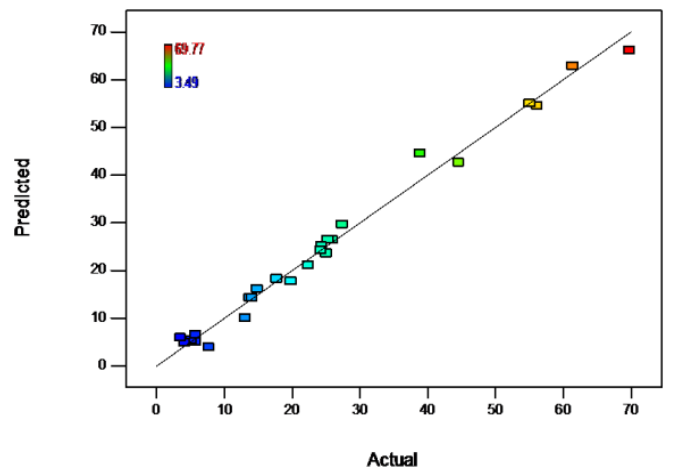

Figure (5.1-2). Predicted values versus the experimental data for (a) mixing time (s) and (b) power consumption (W).

Response surface plots are plotted as a function of two factors at a time while all other factors are kept unchanged. The results of the experimental design for the effect of the rheological parameters and the speed ratio of the impellers on the efficiency of the coaxial mixer generate 3D response surface planes for mixing time and power consumption using quadratic equations [i.e. Eq. (5.1-2) and Eq. (5.1-3)] as shown in Figure (5.1-3) and (5.1-4), respectively. These figures describe the contribution of four variables and their interactions on the mixing time and power consumption. 
(a)

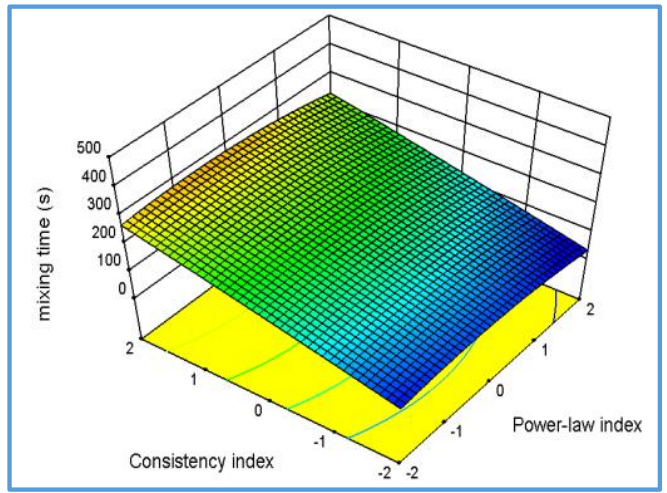

(c)

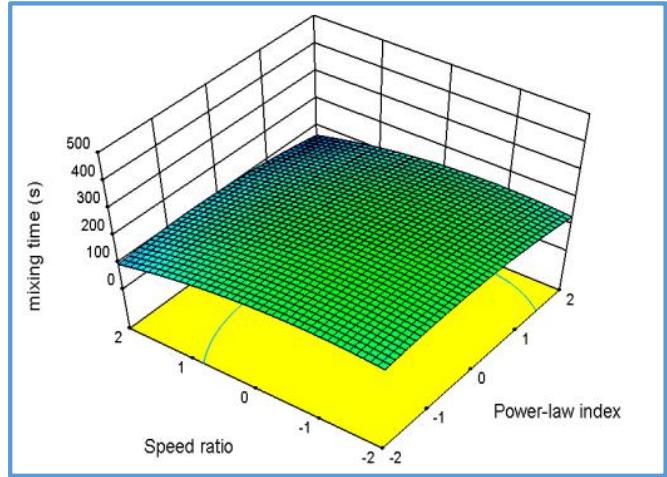

(e)

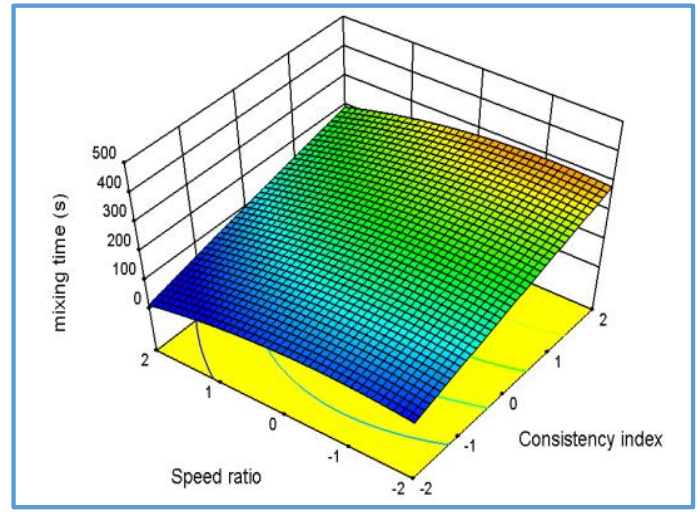

(b)

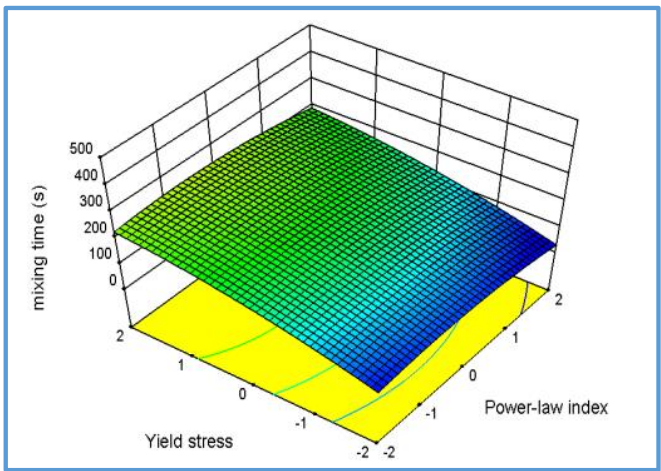

(d)

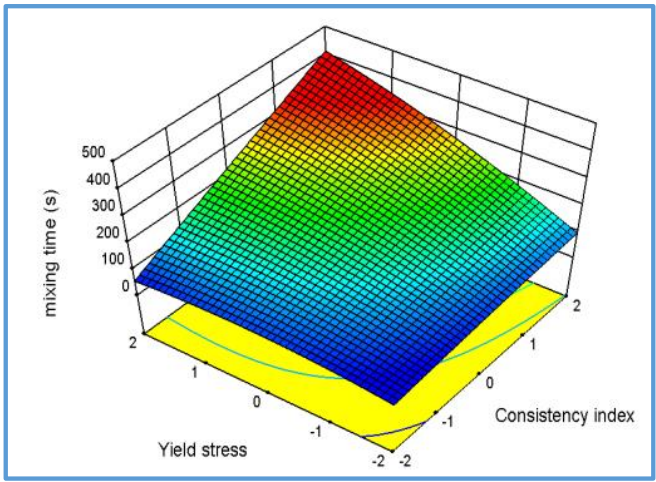

(f)

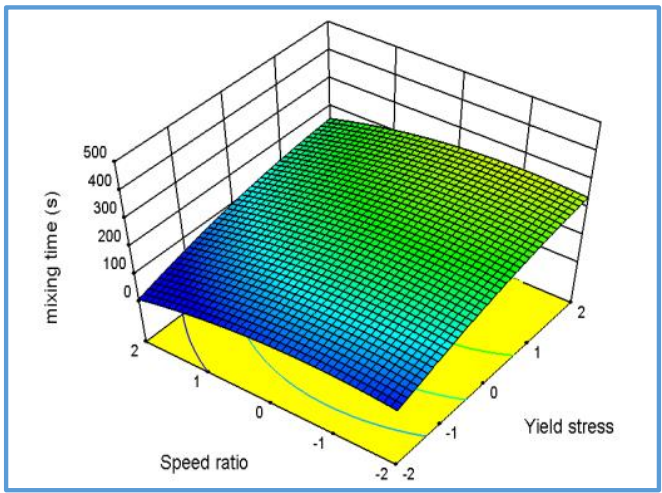

Figure (5.1-3) Response surface methodology (RSM) showing mixing time as a function of two independent variables (other variables were held at their respective center levels): (a) power-law index $(n)$ and consistency index $(K)$, (b) power-law index $(n)$ and yield stress $\left(\tau_{y}\right),(\mathbf{c})$ power-law index $(n)$ and speed ratio $\left(R_{n}\right),(\mathbf{d})$ consistency index $(K)$ and yield stress $\left(\tau_{y}\right),(\mathbf{e})$ consistency index $(K)$ and speed ratio $\left(R_{n}\right)$, and (f) yield stress $\left(\tau_{y}\right)$ and speed ratio $\left(R_{n}\right)$. 
(a)

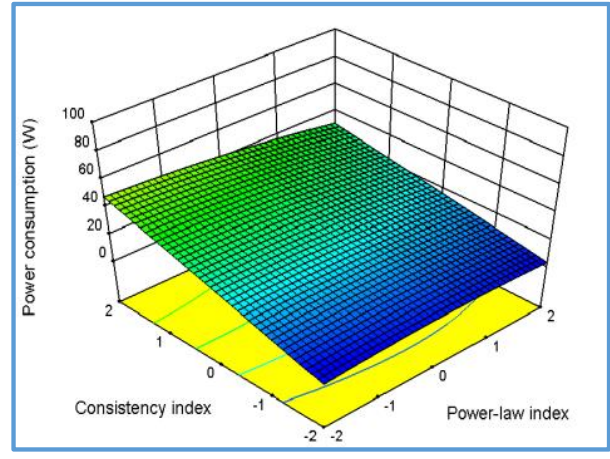

(c)

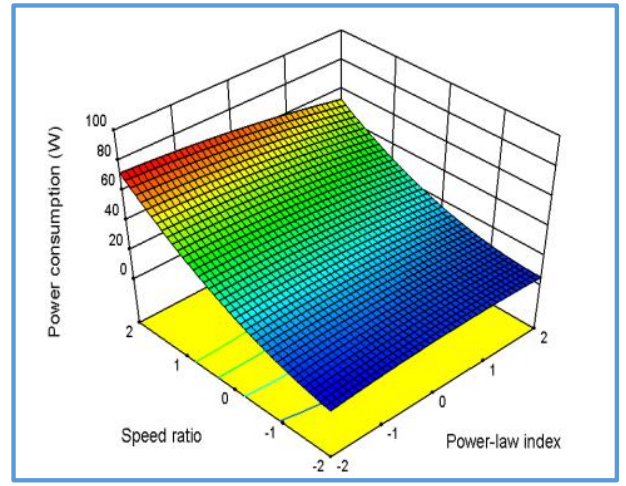

(e)

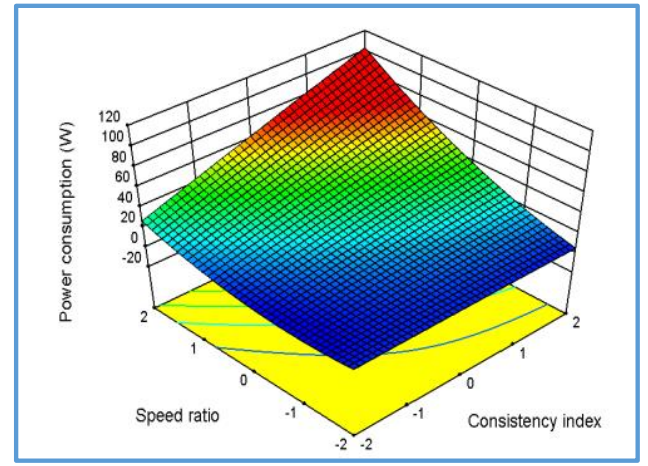

(b)

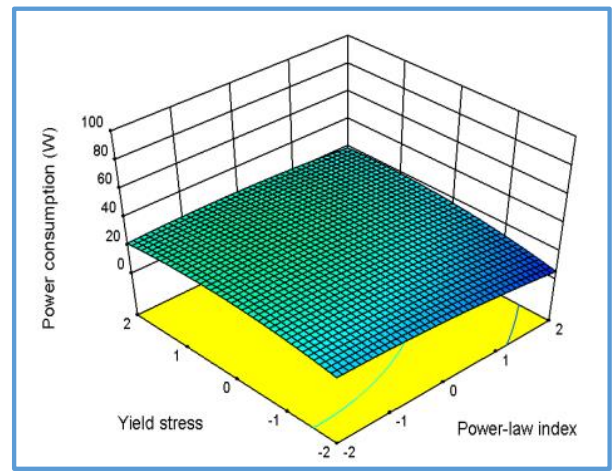

(d)

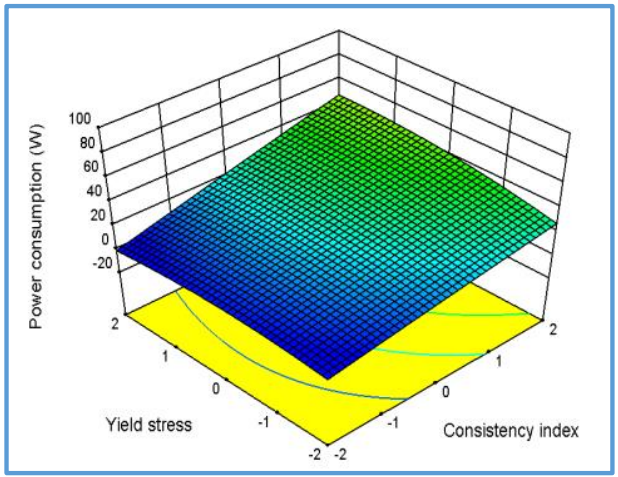

(f)

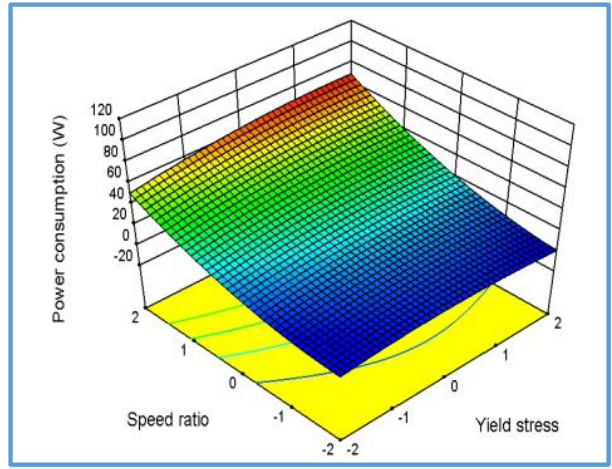

Figure (5.1-4). Response surface methodology (RSM) showing power consumption as a function of two independent variables (other variables were held at their respective center levels): (a) power-law index $(n)$ and consistency index $(K)$, (b)power-law index $(n)$ and yield stress $\left(\tau_{y}\right)$, (c) power-law index $(n)$ and speed ratio $\left(R_{n}\right),(\mathbf{d})$ consistency index $(K)$ and yield stress $\left(\tau_{y}\right),(\mathbf{e})$ consistency index $(K)$ and speed ratio $\left(R_{n}\right)$, and (f) yield stress $\left(\tau_{y}\right)$ and speed ratio $\left(R_{n}\right)$. 
Figures (5.1-3 a, b, c) and Figures (5.1-4 a, b, c) represent the influence of power-law index on the mixing time and power consumption of the Scaba-anchor coaxial agitated system, respectively. These figures show that the power-law index has the least effect (the small slope of RSM planes) on power consumption and mixing time of agitated system among four specified parameters (i.e. power-law index, consistency index, yield stress, and speed ratio). It must be mentioned that for the shear thinning fluid $(n<1)$ when the power-law index is raised, fluid viscosity decreases at a fixed shear rate according to Equation (3.3-1). Figure 5.1-3 (a, b, c) and Figure 5.1-4 (a, b, c) demonstrate that both power consumption and mixing time decreased slightly from 5.69 to $5.12 \mathrm{~W}$ and from 81 to $70 s$ when the power law index was increased from 0.12 to 0.80 at the fixed values of speed ratio, consistency index, and yield stress. To better understand the effect of the power law index on the performance of the mixing system, the viscosity contour plots in vertical cross section were calculated through the validated CFD model as shown in Figure (5.1-5).

(a)
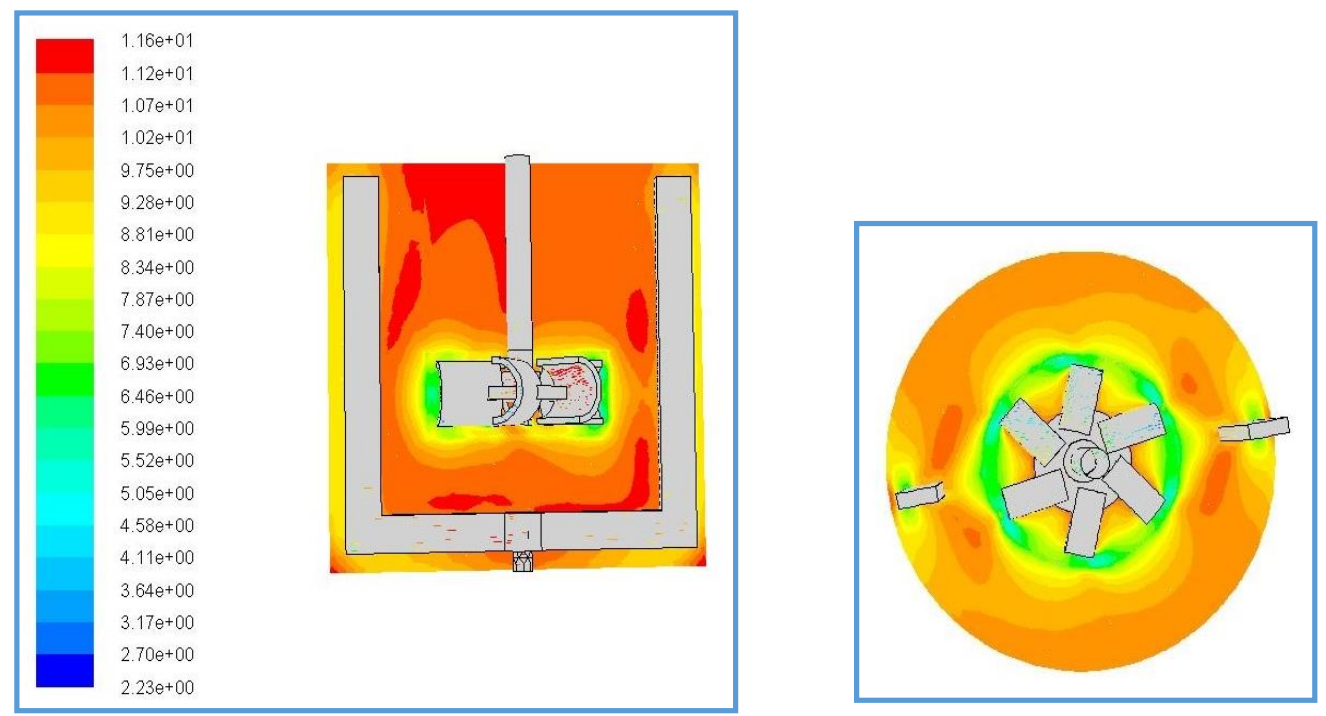
(b)
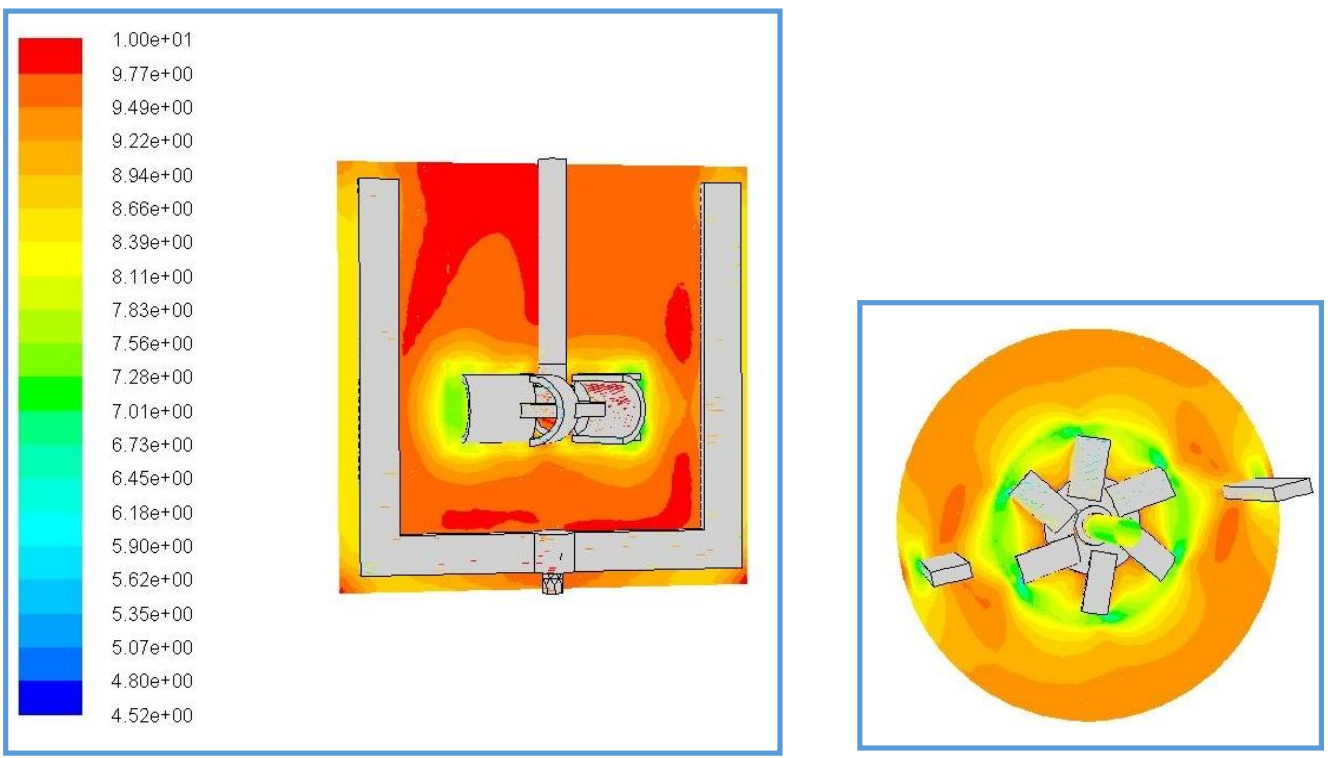

Figure (5.1-5). Viscosity (kg/m-s) contour plots for effect power-law index on the mixing system produced by the central impeller using vertical cross section planes for $1 \%$ xanthan gum concentration agitated at: (a) $n=0.12$ (b) $n=0.8$ (c) at $K=8, \tau_{y}=5.254$, and $R n=5$.

It can be seen when the power law index was increased from $n=0.12$ to $n=0.80$, the average apparent viscosity decreased from 9.36 to $5.48 \mathrm{~kg} / \mathrm{m}$ s. It can be concluded that the fluid viscosity throughout the mixing tank did not change significantly due to the variation of the power-law index for the operating conditions employed in this study.

Figures (5.1-3 a, d, e) and (5.1-4a, d, e) demonstrate the effects of the consistency index on the mixing time and power consumption of the Scaba-anchor coaxial mixer, respectively. It is evident that the power drawn and mixing time increased by an increase in the consistency index $(K)$. One can easily notice that among the investigated variables the consistency index had the most significant influence on the mixing time and power 
consumption among the investigated variables. This can be attributed to the apparent viscosity of the Herschel-Bulkley fluid, which increases by increasing the consistency index. It is also worth noting that the interaction between the consistency index and yield stress is considered as the most important interaction among the investigated variables according to Equations (5.1-2) and (5.1-3).

To visualize the effect of the consistency index, the validated CFD model was used to calculate the fluid flow domain generated by the coaxial mixer as a function of the consistency index at the speed ratio of $5\left(N_{a}=30 \mathrm{rpm}\right.$ and $\left.N_{s}=150 \mathrm{rpm}\right)$ as shown in Figure (5.1-6).

(a)

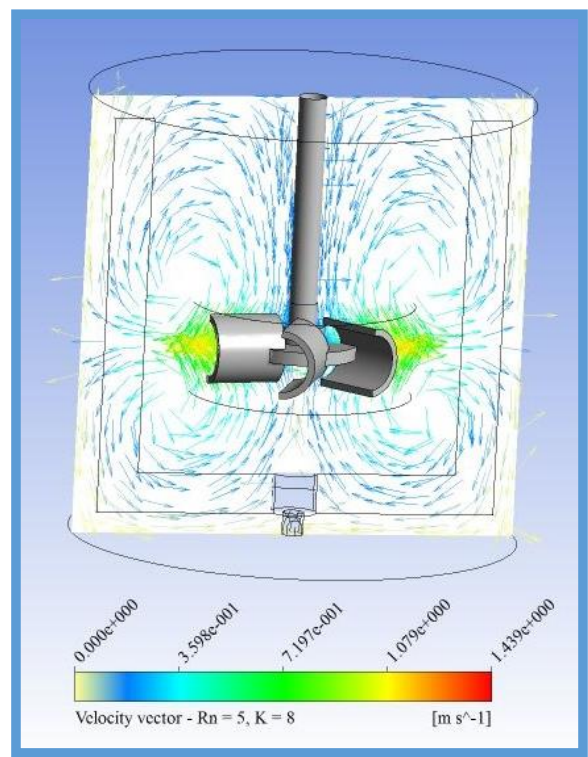

(b)

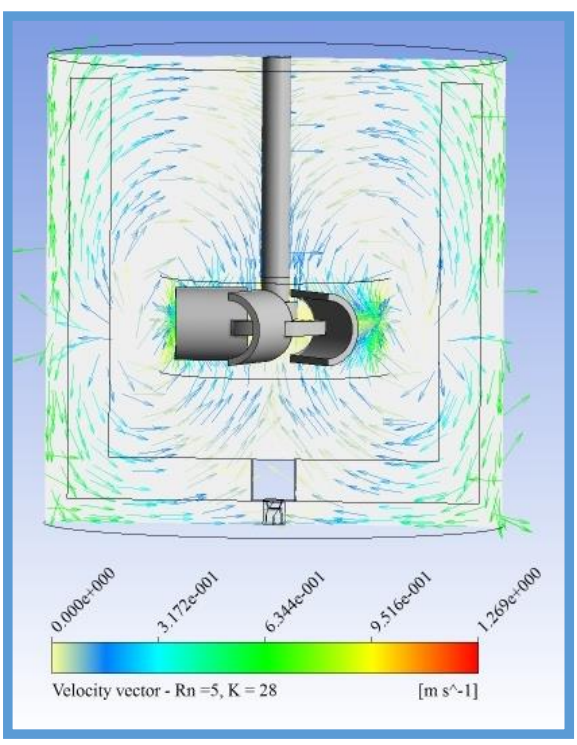

Figure (5.1-6). Velocity $(\mathrm{m} / \mathrm{s})$ vector plots depicting the effect of consistency index on the mixing system: (a) $K=8 \mathrm{Pas}^{\mathrm{n}}$ and (b) $K=28 \mathrm{Pas}^{\mathrm{n}}$ for Scaba-anchor impeller agitated at $N_{a}=30 \mathrm{rpm}, N_{s}=150 \mathrm{rpm}, n=0.12, \tau_{y}=5.254 \mathrm{~Pa}$.

It can be seen that at the higher value of the consistency index, the zones of the stagnant and slowly moving fluid were created below the fluid surface and the near the top shaft. 
It can be noticed that at $K=8 \mathrm{~Pa} \mathrm{~s}^{\mathrm{n}}$, the radial-flow pattern with two circulation loops were clearly formed in the mixing tank. However, by increasing the consistency index form 8 to $28 \mathrm{~Pa} \mathrm{~s}^{\mathrm{n}}$, the radial-flow pattern created by the Scaba impeller diminished and the tangential-flow generated by the anchor impeller became more noticeable, which resulted in the reduction of the mixing intensity at the same impeller speed ratio.

Figures (5.1-3 c, e, f) depict that for a fixed value of consistency index, yield stress, and power-law index, the mixing time reduced with increasing in the speed ratio from $R n=$ 1.7 to $R_{n}=6.0$ for the fixed values of the rheological parameters. Figure $(5.1-4 \mathrm{c}$, e, and f) demonstrates that the power consumption increased with the speed ratio at the given value of the consistency index, power-law index, and yield stress. These figures show that the influence of the speed ratio on the power drawn and mixing time was significant. This was in agreement with that reported by Pakzad et al. (2013a) for the mixing of pseudoplastic fluids with yield stress with the coaxial mixers. The validated CFD model was used to simulate the flow domain as a function of the speed ratio as depicted in Figure (5.1-7). 
(a)

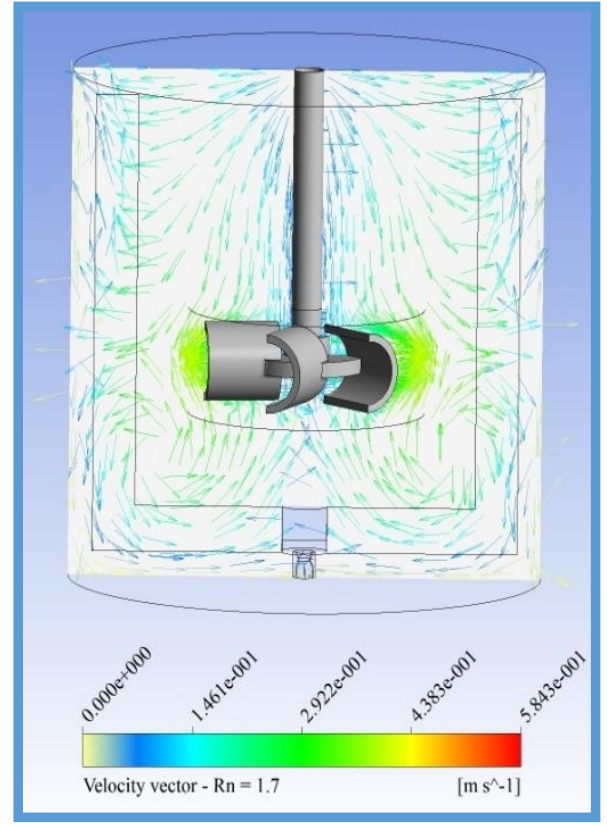

(b)

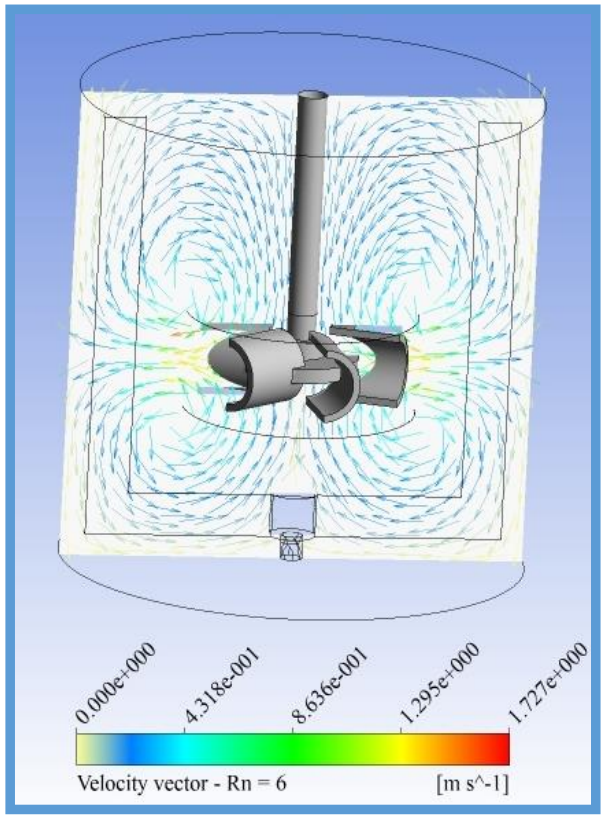

Figure (5.1-7). Velocity $(\mathrm{m} / \mathrm{s})$ vector plots in vertical direction as a function of the speed ratio for the Scaba-anchor impeller at $1.0 \%$ xanthan gum solution, $n=0.12, K=8 \operatorname{Pas}^{\mathrm{n}}$, and $\tau_{y}=5.254 \mathrm{~Pa}:$ (a) $N_{s}=50 \mathrm{rpm}, N_{a}=30 \mathrm{rpm}, R_{n}=1.7$ and (b) $N_{s}=180 \mathrm{rpm}, N_{a}=$ $30 \mathrm{rpm}, R_{n}=6$.

At the lower speed ratio, two low-velocity regions (i.e. weak mixing zones) were formed: one below the central impeller and the other above the central impeller along the central shaft. As it can be noticed, mixing took place between two loops (not within the loops). The flow domain at the low speed ratio of 1.7 was largely dominated by the tangential flow produced by the anchor impeller (Pakzad et al., 2013a). However, with increasing the speed ratio from 1.7 to 6.0 , two circulation loops were generated by the rotation of the Scaba impeller. In fact, at the higher speed ratio, the radial flow generated by the Scaba impeller played the major role while the rotation of the anchor impeller eliminated the formation of the stagnant regions near the wall and the bottom of the vessel. 
The mixing performance was also found to be the function of the yield stress according to the data presented in Figures (5.1-3 b, d, and f) and (5.1-4 b, d, and f). It can be seen that the power consumption and mixing time increased when the yield stress changed from $\tau_{y}$ $=5.25 \mathrm{~Pa}$ to $\tau_{y}=20.60 \mathrm{~Pa}$. This can be attributed to the apparent viscosity of the Herschel-Bulkley fluid, which increases by increasing the yield stress. The influence of yield stress on the mixing time of the non-Newtonian fluids was investigated by EinMozaffari and Upreti (2009). They indicated that the yield stress had a considerable effect on the mixing of yield-pseudoplastic fluids with the single impeller. The validated CFD model was applied to simulate the 3D streamline plots for the fluid yield stresses of 5.25 $\mathrm{Pa}$ and $20.60 \mathrm{~Pa}$ at the speed ratio of 5 as shown in Figure (5.1-8).

(a)

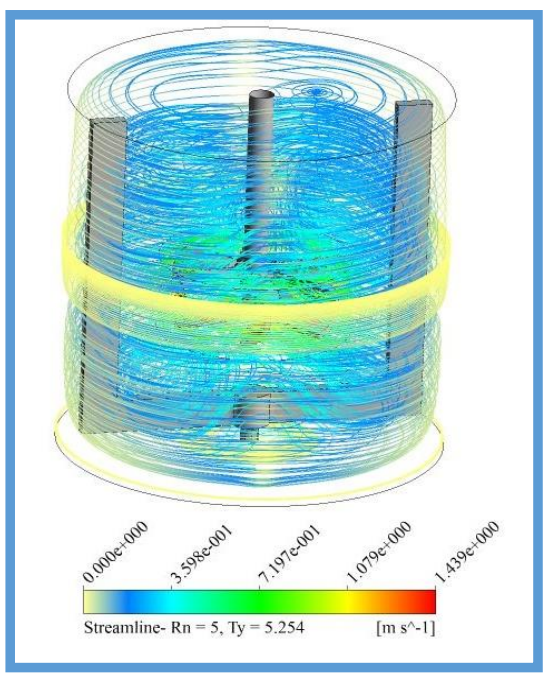

(b)

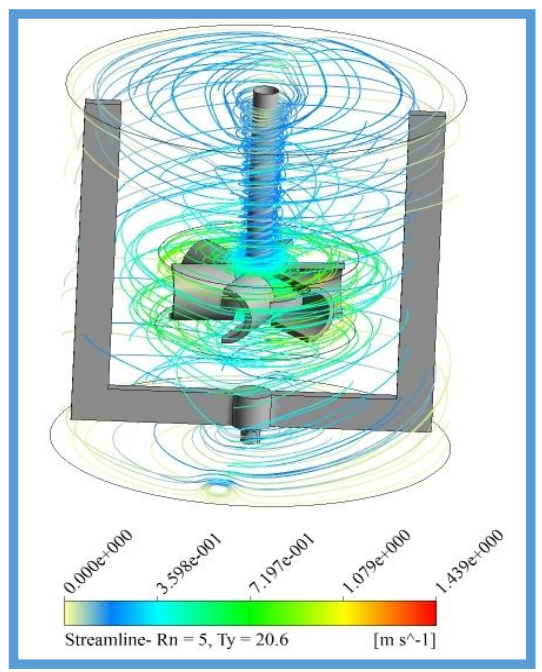

Figure (5.1-8). Three dimensional streamlines (3D) plots produced by the central impeller using vertical cross section planes for $1 \%$ xanthan gum concentration agitated for: (a) $\tau_{y}=5.254 \mathrm{~Pa}, R n=5$, (b) $\tau_{y}=20.6 \mathrm{~Pa}, R n=5$ at $K=8 \mathrm{~Pa} \mathrm{~s}^{\mathrm{n}}, n=0.12$.

It can be noticed that the mixing intensity and effectiveness decreased when the fluid yield stress increased from 5.25 to $20.60 \mathrm{~Pa}$. In fact, increasing the yield stress resulted in 
the formation of the stagnant and slowly moving fluid zones in the mixing vessel. Thus, more power consumption is required to eliminate these undesired zones and to enhance the mixing quality.

In addition to the techniques available for measuring the mixing time, several empirical correlations have also been reported in the literature for estimating the mixing time. In these models, experimental data are correlated with the operating variables. Moo-Young et al. (1972) developed a model based on the dependency of dimensionless mixing time $\left(N t_{m}\right)$ with Reynolds number $\left(R_{e}\right)$ which is applicable for both Newtonian and nonNewtonian fluids:

$$
N t_{m}=a R_{e}^{b}
$$

where $a$ and $b$ are constants. Figure (5.1-9) represents dependency of dimensionless mixing time and generalized Reynolds number as a function of consistency index [Figure (5.1-9) a], power-law index [Figure (5.1-9) b], and yield stress [Figure (5.1-9) c)] at five speed ratios. The figures show that dimensionless mixing time decreased with Reynolds number due to consistency index $(K)$ and yield $\operatorname{stress}\left(\tau_{y}\right)$, however, the effect of powerlaw index $(n)$ was not significant. 
(a)

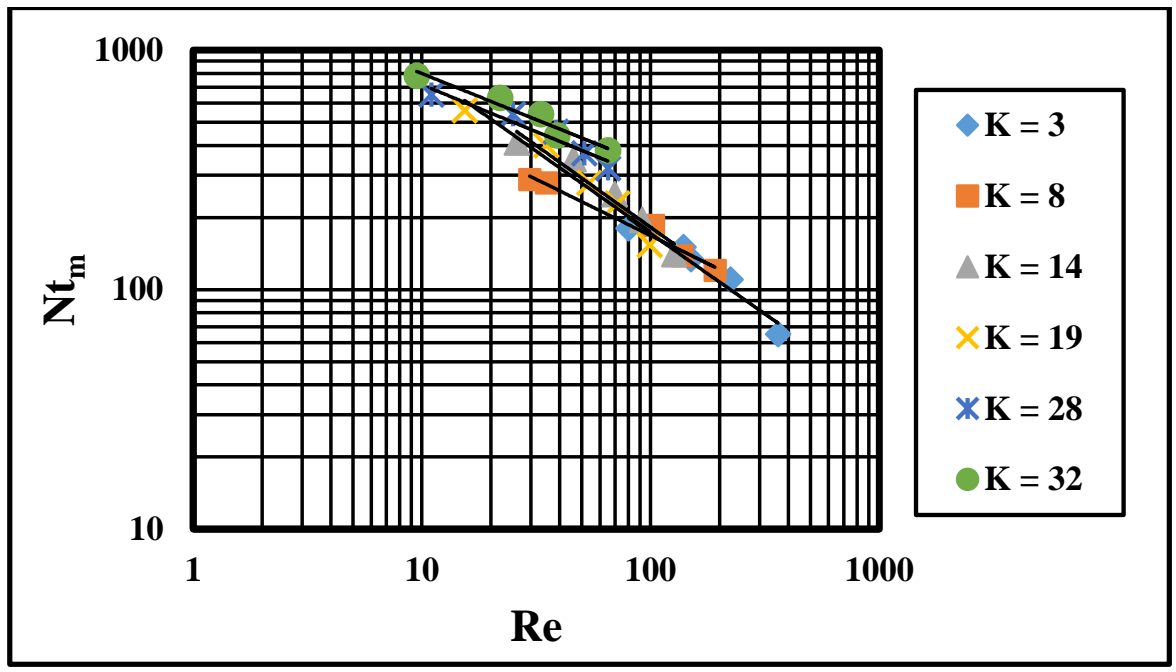

(b)

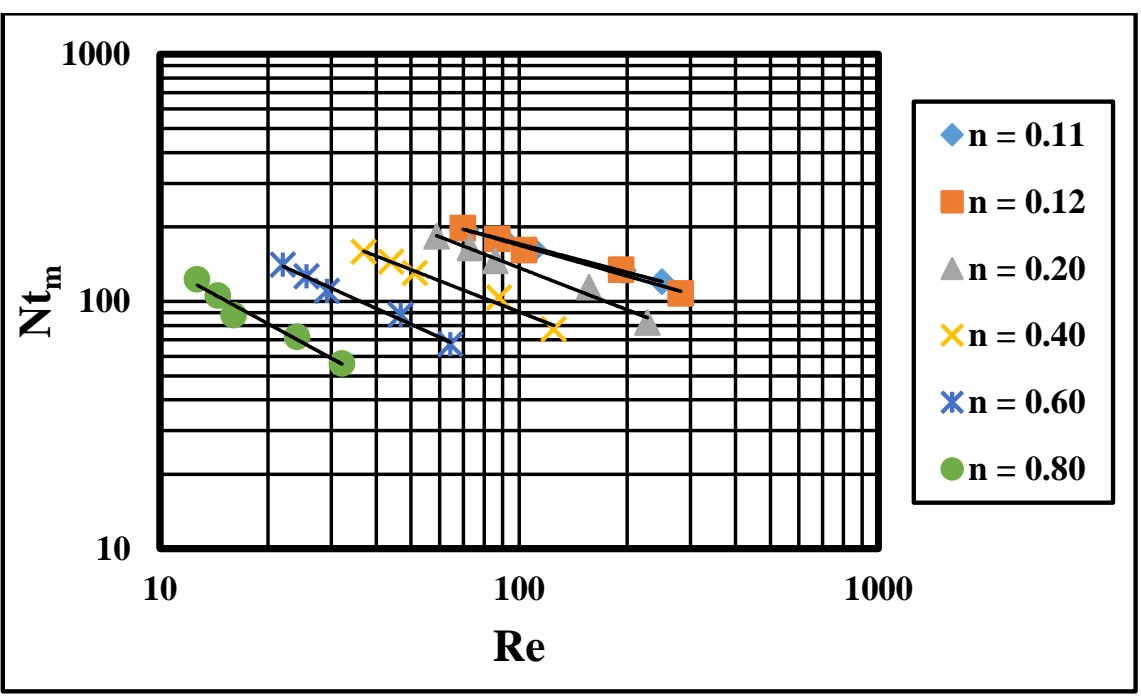


(c)

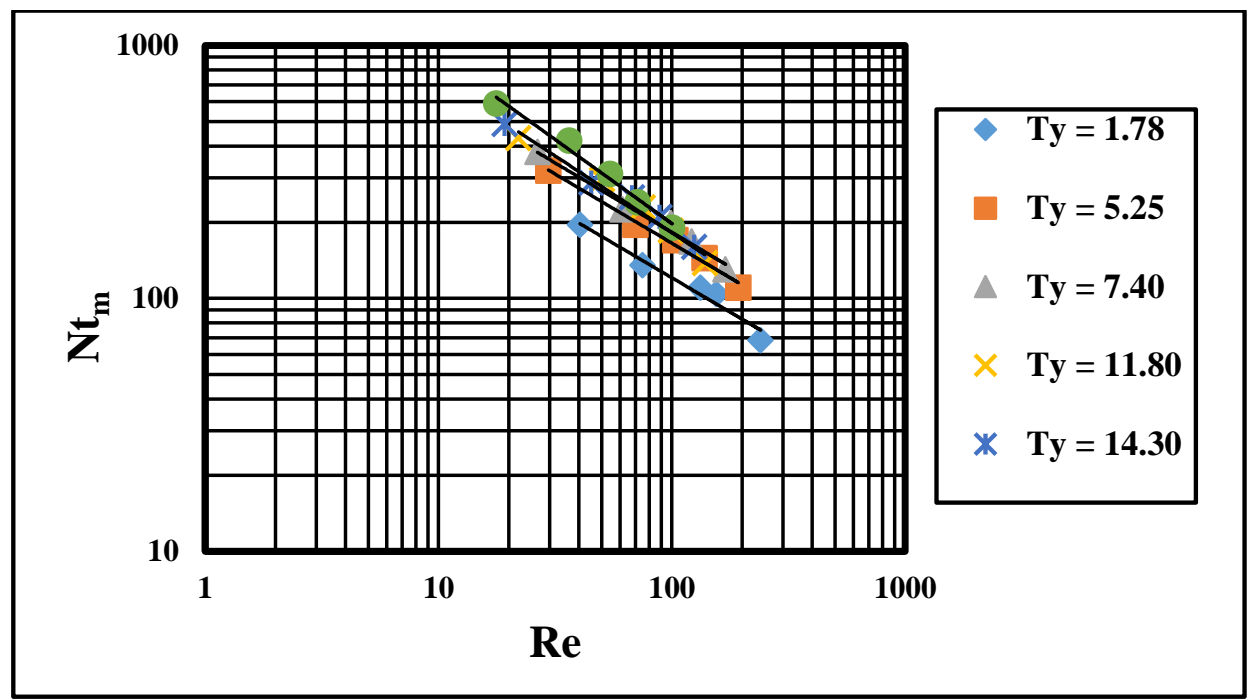

Figure (5.1-9). Correlation between dimensionless mixing time and generalized Reynolds number for the effect of: (a) consistency index $(K)$, (b) power-law-index $(n)$, and $(\mathbf{c})$ yield stress $\left(\tau_{\mathrm{y}}\right)$ at five speed ratio for $1.0 \%$ xanthan gum solution.

The regression analysis proved that the data shown in Figures [(5.1-9) a, b, and c] were fitted well to Equation (5.1-4). The values of $a$ and $b$ were determined and listed in [Tables (5.1-5), (5.1-6) and (5.1-7)].

Table (5.1-5). Coefficients of $N . t_{m}$ vs. $R e$ as a function of the speed ratio when the consistency index $(K)$ changes from 3 to 32 Pa.s ${ }^{n}$.

\begin{tabular}{ccccc}
\hline Consistency index & $\begin{array}{c}\text { Range of } \\
\text { Re }\end{array}$ & $a$ & $b$ & $R^{2}$ \\
\hline$K=3$ & $80-360$ & 3785.3 & -0.672 & 0.9346 \\
$K=8$ & $29-191$ & 1460.3 & -0.469 & 0.9729 \\
$K=14$ & $26-127$ & 4223.1 & -0.683 & 0.9456 \\
$K=19$ & $15-99$ & 3893.5 & -0.675 & 0.9530 \\
$K=28$ & $11-65$ & 1767.1 & -0.392 & 0.9354 \\
$K=32$ & $9-65$ & 1933.9 & -0.385 & 0.9470 \\
\hline
\end{tabular}


Table (5.1-6). Coefficients of $N . t_{m}$ vs. $R e$ as a function of the speed ratio when the power-law index $(n)$ changes from 0.12 to 0.80 .

\begin{tabular}{ccccc}
\hline $\begin{array}{c}\text { Power-law } \\
\text { index }\end{array}$ & Range of Re & $a$ & $b$ & $R^{2}$ \\
\hline$n=0.11$ & $71-250$ & 996.3 & -0.383 & 0.9967 \\
$n=0.12$ & $69-282$ & 1134.8 & -0.414 & 0.9849 \\
$n=0.20$ & $58-228$ & 1852.1 & -0.566 & 0.9809 \\
$n=0.40$ & $36-125$ & 1238.4 & -0.568 & 0.9841 \\
$n=0.60$ & $22-65$ & 1061.4 & -0.655 & 0.9856 \\
$n=0.80$ & $12-33$ & 871.8 & -0.792 & 0.9675 \\
\hline
\end{tabular}

Table (5.1-7). Coefficients of $N . t_{m}$ vs. $R e$ as a function of the speed ratio when the yieldstress $\left(\tau_{\mathrm{y}}\right)$ changes from 1.78 to $20.60 \mathrm{~Pa}$.

\begin{tabular}{ccccc}
\hline Yield stress & Range of Re & $a$ & $b$ & $R^{2}$ \\
\hline$\tau_{y}=1.78$ & $40-241$ & 1473.4 & -0.543 & 0.9624 \\
$\tau_{y}=5.25$ & $29-193$ & 2050.6 & -0.547 & 0.9885 \\
$\tau_{y}=7.40$ & $26-171$ & 2286.6 & -0.549 & 0.9809 \\
$\tau_{y}=11.80$ & $22-139$ & 2971.3 & -0.607 & 0.9777 \\
$\tau_{y}=14.30$ & $14-125$ & 2687.2 & -0.576 & 0.9887 \\
$\tau_{y}=20.60$ & $17-101$ & 4214.1 & -0.664 & 0.9823 \\
\hline
\end{tabular}

To determine the efficiency of the mixing system, Moo-Yong et al. (1972) and Nienow (1997) developed a correlation in terms of mixing time and power drawn per unit volume $(P / V)$ (called specific power consumption) as follows:

$$
t_{m}=\mathrm{c}\left(\frac{P}{V}\right)^{d}
$$

where $c$ and $d$ are considered as constant. Figure (5.1-10) shows the variation of mixing time $\left(t_{m}\right)$ versus $P / V$ as a function of consistency index [Figure (5.1-10)a], power-law index [Figure (5.1-10) b], and yield stress [Figure (5.1-10) c] at five speed ratios. 
(a)

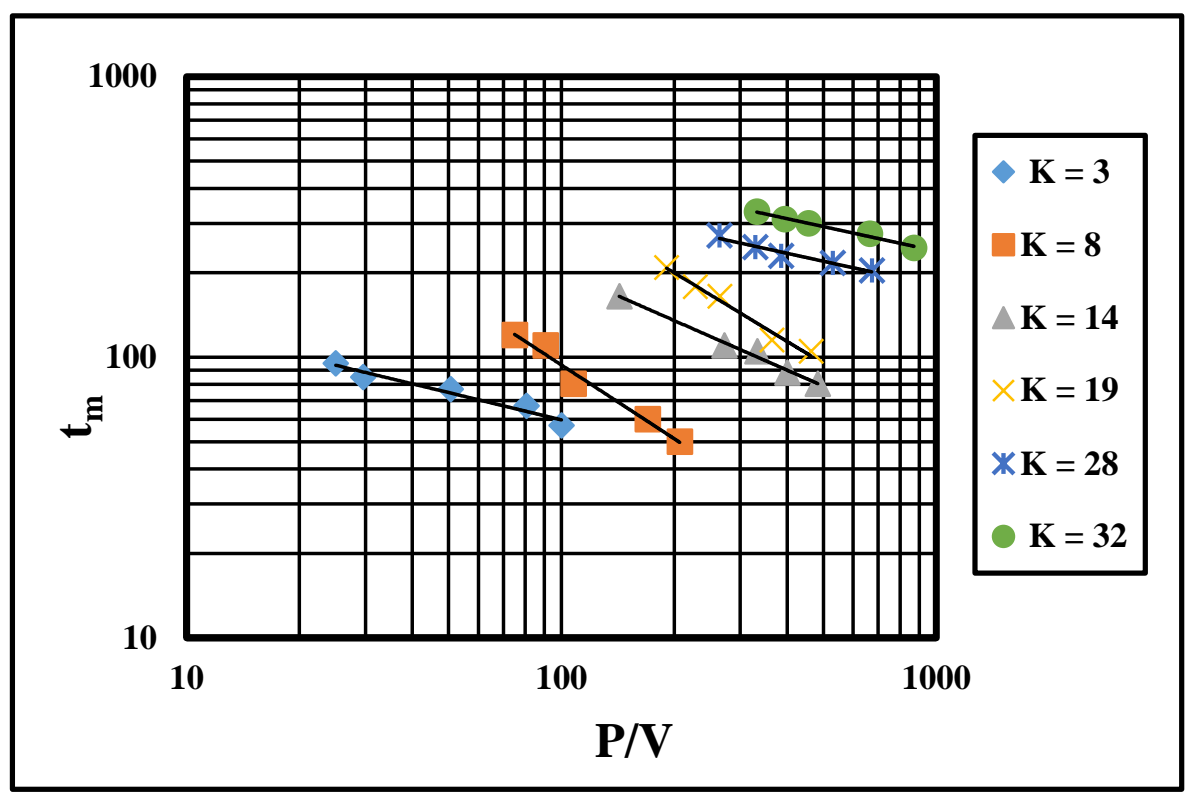

(b)

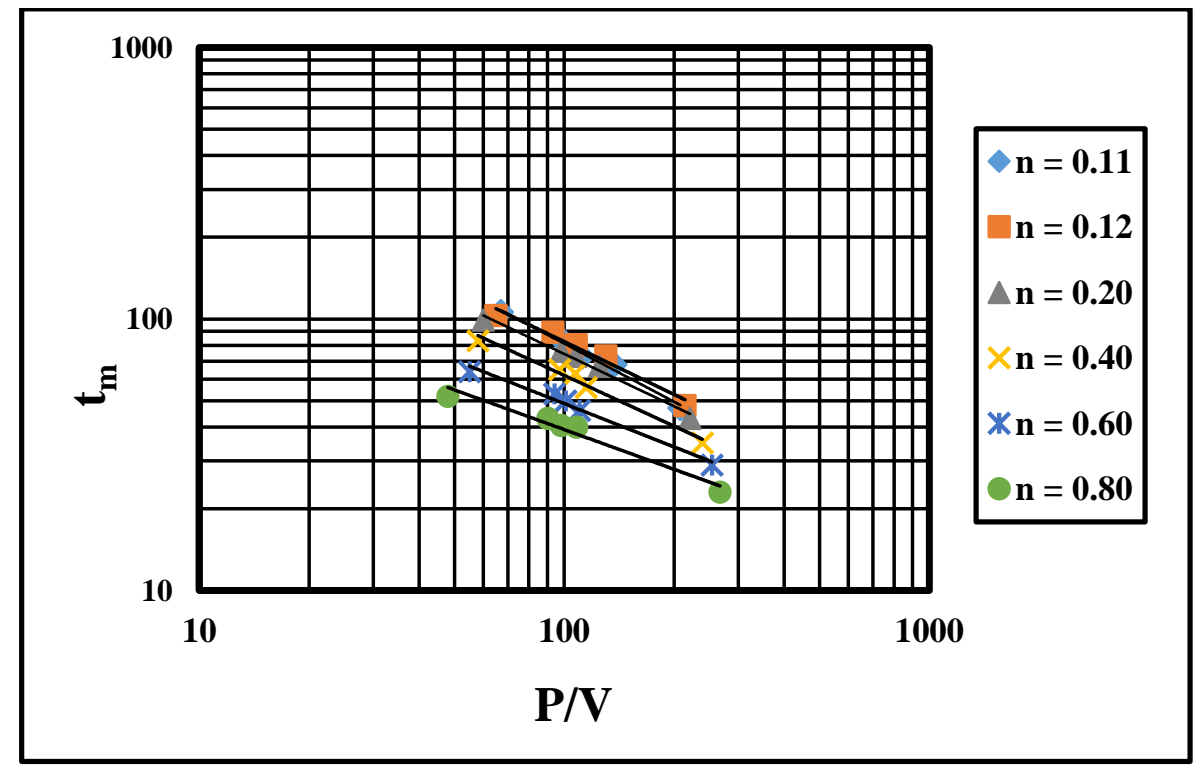


(c)

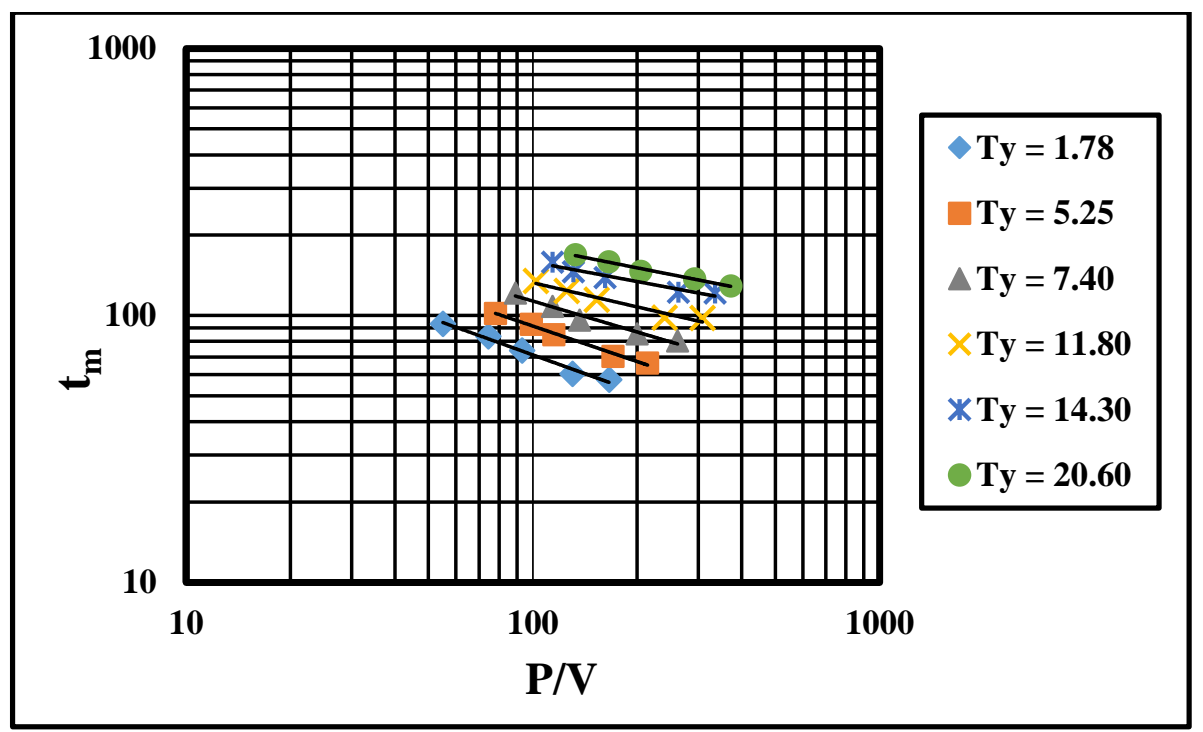

Figure (5.1-10). Correlation between mixing time and specific power consumption for the effect of: (a) consistency index $(K)$, (b) power-law-index $(n)$, and (c) yield stress $\left(\tau_{y}\right)$ at five speed ratio for $1.0 \%$ xanthan gum solution.

The obtained regression coefficients of $c$ and $d$, and the correlation coefficients $\left(R^{2}\right)$ have been reported in Tables (5.1-8), (5.1-9), and (5.1-10). The correlation coefficients show that the mixing time values were fitted well to Equation (5.1-5).

Table (5.1-8). Coefficients of $t_{m}$ vs. $P / V$ as a function of the consistency index $(K)$.

\begin{tabular}{ccccc}
\hline $\begin{array}{c}\text { Consistency } \\
\text { index }\end{array}$ & $\begin{array}{c}\text { Range of } \\
\text { Re }\end{array}$ & $c$ & $d$ & $R^{2}$ \\
\hline$K=3$ & $25-100$ & 265.0 & -0.323 & 0.9544 \\
$K=8$ & $75-207$ & 5243.3 & -0.874 & 0.9766 \\
$K=14$ & $143-483$ & 3030.5 & -0.587 & 0.9892 \\
$K=19$ & $190-463$ & 14815.0 & -0.813 & 0.9791 \\
$K=28$ & $264-673$ & 1354.0 & -0.292 & 0.9659 \\
$K=32$ & $332-872$ & 1778.9 & -0.291 & 0.9834 \\
\hline
\end{tabular}


Table (5.1-9). Coefficients of $t_{m}$ vs. $P / V$ as a function of the power-law index $(n)$.

\begin{tabular}{ccccc}
\hline $\begin{array}{c}\text { Power-law } \\
\text { index }\end{array}$ & $\begin{array}{c}\text { Range of } \\
\text { Re }\end{array}$ & $c$ & $d$ & $R^{2}$ \\
\hline$n=0.11$ & $67-208$ & 2136.0 & -0.710 & 0.9883 \\
$n=0.12$ & $65-214$ & 1629.6 & -0.648 & 0.9703 \\
$n=0.20$ & $60-222$ & 1414.4 & -0.640 & 0.9847 \\
$n=0.40$ & $58-239$ & 1081.8 & -0.621 & 0.9807 \\
$n=0.60$ & $55-254$ & 562.5 & -0.531 & 0.9821 \\
$n=0.80$ & $48-267$ & 367.6 & -0.486 & 0.9618 \\
\hline
\end{tabular}

Table (5.1-10). Coefficients of $t_{m}$ vs. $P / V$ as a function of the yield stress $\tau_{y}$.

\begin{tabular}{ccccc}
\hline Yield stress & Range of Re & $c$ & $d$ & $R^{2}$ \\
\hline$\tau_{y}=1.78$ & $55-166$ & 612.9 & -0.467 & 0.9838 \\
$\tau_{y}=5.25$ & $78-215$ & 697.6 & -0.442 & 0.9904 \\
$\tau_{y}=7.40$ & $89-262$ & 665.9 & -0.385 & 0.9703 \\
$\tau_{y}=11.80$ & $102-309$ & 530.7 & -0.301 & 0.9556 \\
$\tau_{y}=14.30$ & $114-336$ & 485.4 & -0.243 & 0.9473 \\
$\tau_{y}=20.60$ & $132-372$ & 588.5 & -0.257 & 0.9855 \\
\hline
\end{tabular}

\subsubsection{Conclusion}

The effect of the rheological properties (consistency index $(K)$, power-law-index $(n)$, yield stress $\left.\left(\tau_{y}\right)\right)$ of the Herschel-Bulkley fluids on the mixing performance of a coaxial mixing system (Scaba-anchor) was investigated in terms of the mixing time and power consumption at five speed ratios for the co-rotating mode using the electrical resistance tomography (ERT), computational fluid dynamics (CFD), and design of experiments (DOE) combined with the response surface methodology (RSM). The results of both numerical and experimental approaches demonstrated that the power drawn and mixing time of the Scaba-anchor coaxial mixer increased when the consistency index and yield stress were raised. However, the effect of power-law index on the mixing time and power 
consumption was not significant. Design of experiments (DOE) and response surface methodology (RSM) were utilized to investigate the interaction between the independent variables. Data analysis showed that the interaction between the consistency index and yield stress was the most important interaction. The RSM analysis also revealed that the consistency index and speed ratio had the major influence on the mixing efficiency of the coaxial mixing system. Finally, mixing times computed through validated CFD model were correlated well with specific power consumption and Reynolds number for the coaxial system. 


\subsection{Investigation on Hydrodynamic Performances of Coaxial Mixers in Agitation of Yield-Pseudoplasitc Fluids: Single and Double Central Impellers in Combination with the Anchor}

\subsubsection{Introduction}

The focus of the present study was to compare the hydrodynamic performances of two coaxial mixers consisting of the single and double Scaba impellers in combination with the anchor [ Figure (5.2-1)] in the agitation of the xanthan gum solution (non-Newtonian yield-pseudoplastic fluids) in the laminar-transitional regime, both numerically and experimentally. The 3D numerical simulations of flow domain produced by these two types of the coaxial mixers was made by the CFD. The correlations proposed by Pakzad et al. (2013b) and Bao et al. (2011) were applied to obtain the power curves for the coaxial mixers. In this work, the performances of the coaxial mixers were evaluated in terms of mixing time, fluid characterization, and power consumption. The experimental conditions for this study are listed in Table (5.2-1). 

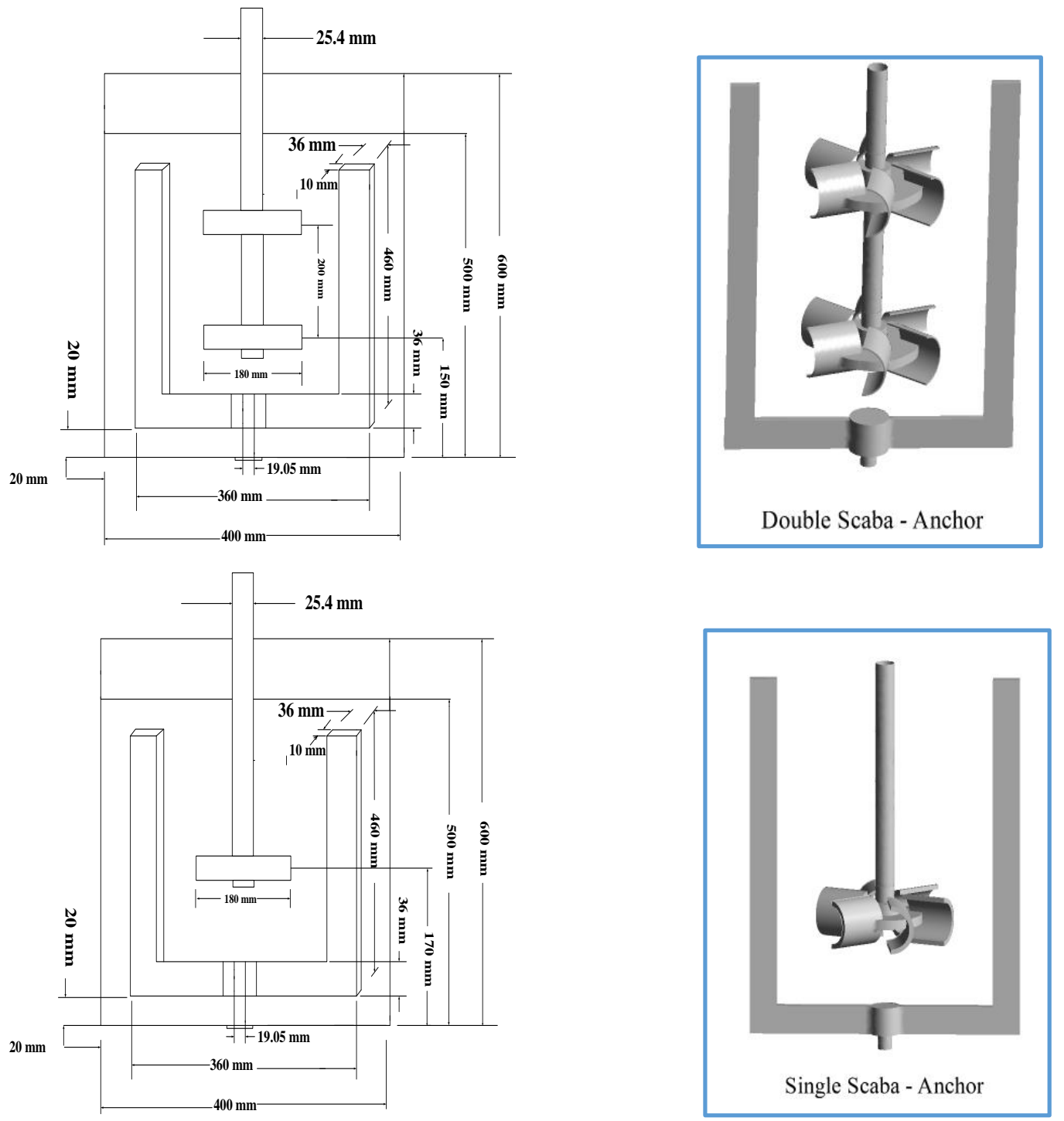

Figure (5.2-1). Configurations of the coaxial mixers used in this study.

Table (5.2-1). The experimental conditions.

\begin{tabular}{cccc}
\hline Speed ratio & Low-speed shaft & $\begin{array}{c}\text { High- speed } \\
\text { shaft }\end{array}$ & $\operatorname{Re}$ \\
$\mathrm{N}_{\mathrm{c}} / N_{a}$ & $N_{a}(\mathrm{rpm})$ & $N_{c}(\mathrm{rpm})$ & $(-)$ \\
\hline 3 & $15-50$ & $50-170$ & $5-110$ \\
6 & $5-25$ & $50-170$ & $5-110$ \\
8 & $5-20$ & $50-170$ & $5-110$ \\
\hline
\end{tabular}

$N_{c}$ : Rotational speed of the Scaba, $N_{a}$ : rotational speed of the anchor 
The numerical simulations of the models were performed by computational fluid dynamic (CFD). The CFD model in this work was then validated by the experimentally determined mixing time and torque measurements for $0.5 \%$ xanthan gum solution at different speed ratios. A good agreement (maximum relative error $<5 \%$ ) was achieved between the computed torque and mixing times and experimentally obtained values as shown in Table (5.2-2). The normalized tracer concentrations versus time at two different locations (Point A and Point B) computed through the CFD model were also compared to those obtained from the tomography data [Figure (5.2-2)]. These results showed reasonable agreement between the CFD and tomography data.

Table (5.2-2). Computed and measured torque and mixing time for $0.5 \%$ xanthan gum solution agitated at $R_{n}=8$ by the Scaba-anchor coaxial mixer.

\begin{tabular}{|c|c|c|c|c|c|c|c|}
\hline & & $\begin{array}{c}\text { Measured } \\
\text { Torque }\end{array}$ & $\begin{array}{c}\text { Computed } \\
\text { Torque }\end{array}$ & $\begin{array}{c}\text { Relative } \\
\text { Error }\end{array}$ & $\begin{array}{c}\text { Measured } \\
\text { Mixing } \\
\text { Time } \\
\end{array}$ & $\begin{array}{c}\text { Computed } \\
\text { Mixing } \\
\text { Time } \\
\end{array}$ & $\begin{array}{c}\text { Relative } \\
\text { Error }\end{array}$ \\
\hline No & $\begin{array}{l}\text { Scaba- } \\
\text { Anchor }\end{array}$ & (N.m) & (N.m) & $(\%)$ & $(\min )$ & $(\min )$ & $(\%)$ \\
\hline \multirow[b]{2}{*}{1} & Scaba & 0.317 & 0.328 & 3.4 & \multirow[b]{2}{*}{8.31} & \multirow[b]{2}{*}{8.74} & \multirow[b]{2}{*}{4.9} \\
\hline & Anchor & & 0.426 & 3.3 & & & \\
\hline \multirow[b]{2}{*}{2} & Scaba & 0.462 & 0.483 & 4.4 & \multirow[b]{2}{*}{7.36} & \multirow[b]{2}{*}{7.65} & \multirow[b]{2}{*}{3.8} \\
\hline & & 0.349 & 0.365 & 4.4 & & & \\
\hline \multirow{2}{*}{3} & Scaba & 0.579 & 0.607 & 4.6 & \multirow{2}{*}{6.82} & \multirow{2}{*}{7.16} & \multirow{2}{*}{4.7} \\
\hline & Anchor & 0.284 & 0.298 & 4.7 & & & \\
\hline \multirow{2}{*}{4} & Scaba & 0.659 & 0.684 & 3.7 & \multirow{2}{*}{5.66} & \multirow{2}{*}{5.94} & \multirow{2}{*}{4.8} \\
\hline & Anchor & 0.237 & 0.246 & 3.7 & & & \\
\hline \multirow{2}{*}{5} & Scaba & 0.760 & 0.798 & 4.8 & \multirow{2}{*}{5.29} & \multirow{2}{*}{5.29} & \multirow{2}{*}{4.3} \\
\hline & Anchor & 0.174 & 0.183 & 4.8 & & & \\
\hline
\end{tabular}


(a)

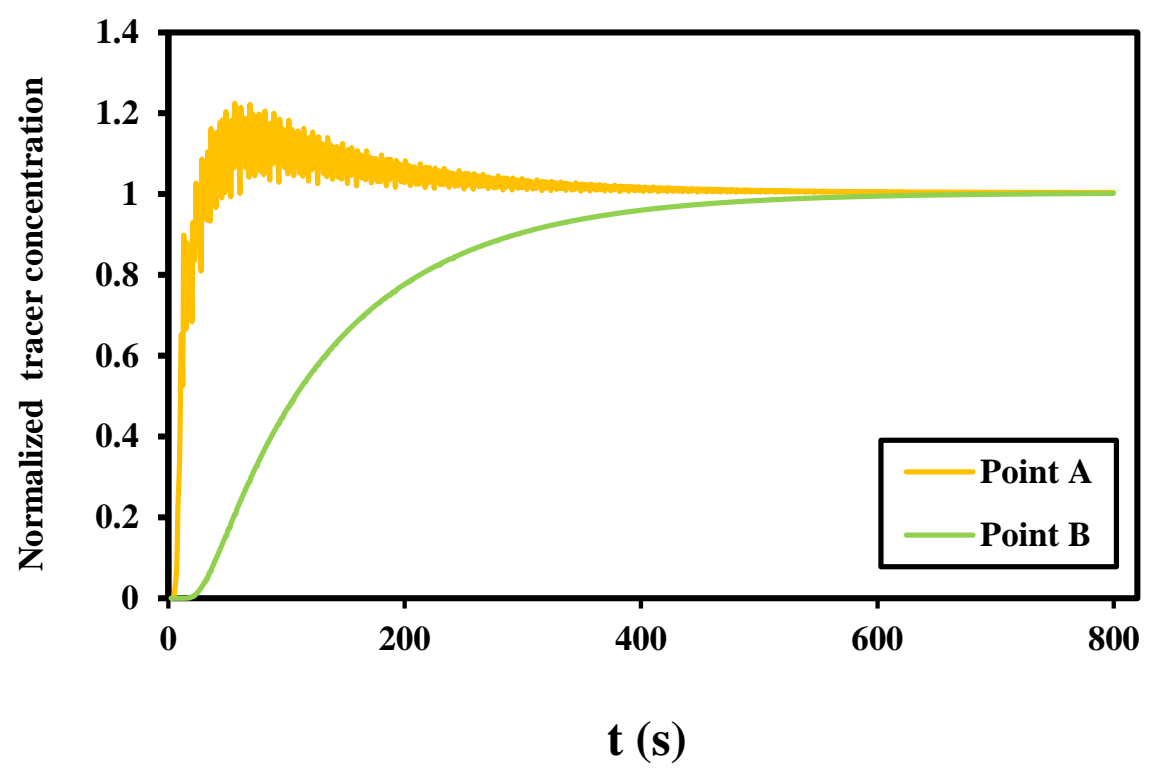

(b)

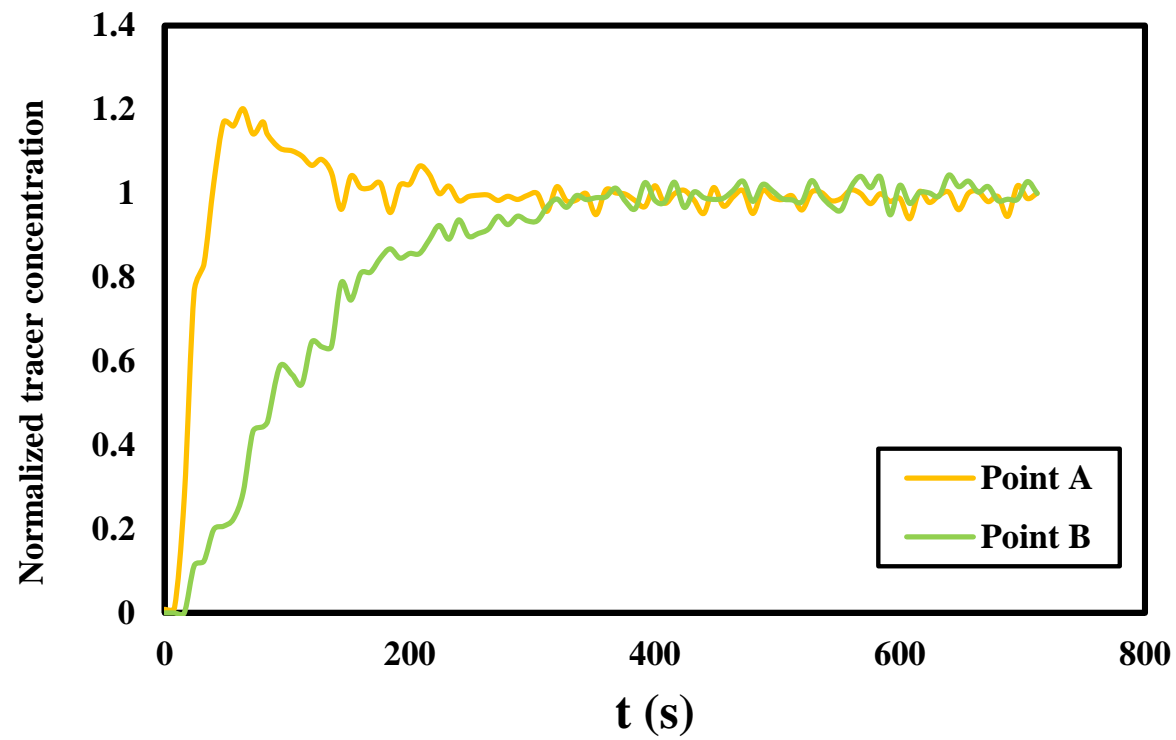

Figure (5.2-2). Normalized tracer concentration versus time at Point $\mathrm{A}(x=0.13, y=$ 0.03 , and $Z=0.360 \mathrm{~m})$ and Point $\mathrm{B}(x=0.00, y=0.00$, and $z=0.140 \mathrm{~m})$ for the DSAC mixer at $R_{n}=8\left(N_{s}=112 \mathrm{rpm}, N_{\mathrm{a}}=14 \mathrm{rpm}\right)$ and $0.5 \%$ xanthan gum solution: (a) CFD results and (b) ERT results. 


\subsubsection{Results and Discussion}

\subsubsection{Power Consumption}

As depicted in Figure [(5.2-1)], a coaxial mixer comprised of two central Scaba impellers $\left(D_{1 s}=D_{2 \mathrm{~s}}=0.18 \mathrm{~m}\right)$ mounted on the same shaft and an anchor impeller $\left(D_{a}=0.36 \mathrm{~m}\right)$ installed on another shaft was used in this work. To obtain the power curve (power number vs. Reynolds number) for such a coaxial mixer with the multiple central impellers, the approaches proposed by Bao et al. (2011) [Equations (5.2-1), (5.2-2) and (5.2-3)]:

$$
\begin{aligned}
& N^{\prime}= \begin{cases}N_{c}+\frac{N_{a}}{R_{n}}: \text { counter }- \text { rotating } \\
N_{c}-\frac{N_{a}}{R_{n}}: \quad \text { co }- \text { rotating }\end{cases} \\
& N p=\frac{P}{\rho N^{\prime 3} D^{5}}=\frac{P_{\text {tot }}}{\rho\left(N_{c} \pm \frac{N_{a}}{R_{n}}\right)^{3} D_{c}{ }^{5}} \\
& \operatorname{Re}=\frac{\rho N^{\prime} D^{2}}{\mu}=\frac{\left(N_{c} \pm \frac{N a}{R_{n}}\right)^{3} D_{c}{ }^{2} \rho}{K K_{s}{ }^{n-1}}
\end{aligned}
$$

and Pakzad et al. (2013 b) [Equations (5-2.4), (5.2-5) and (5.2-6)] were applied to calculate the Reynolds and power numbers. It must be mentioned that $K_{s}$ value equal to 10.5 was considered for the Scaba impeller (Pakzad et al., 2008b).

$$
\begin{gathered}
N^{\prime}=N_{c}+f_{p(a)} N_{a} \\
N_{p(\text { coaxial })}=\frac{P}{\rho N^{\prime 3} D^{5}}=\frac{P_{\text {tot }}}{\rho\left(N_{c}+f_{p(a)} N_{a}\right)^{3} D_{c}^{5}}
\end{gathered}
$$




$$
R e=\frac{\rho N^{\prime} D^{2}}{\mu}=\frac{K_{S(c)}\left(N_{c}+f_{p(a)} N_{a}\right)^{2} D_{c}{ }^{2} \rho}{\left[\tau_{y}+K\left(K_{S(c)}\right)^{n}\left(N_{C}+f_{p(a)} N_{a}\right)^{n}\right]}
$$

It can be noted in Figure (5.2-3) a that all curves generated by Pakzad et al. model (2013b) at different speed ratios $\left(R_{n}=3,6\right.$ and 8$)$ for $1.0 \%$ xanthan gum concentration collapsed to one curve in the co-rotating mode. In contrast, the power plots generated using Bao et al. (2011) model in Figure (5.2-3) b collapsed to one curve at the speed ratios higher than five $\left(R_{n}=6\right.$ and 8$)$ while the power curves did not overlap reasonably at the speed ratio lower than $5\left(R_{n}=3\right)$.

(a)

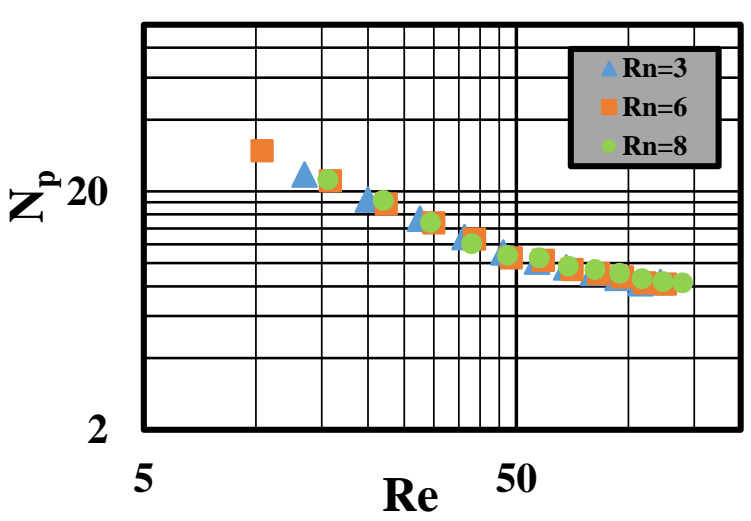

(b)

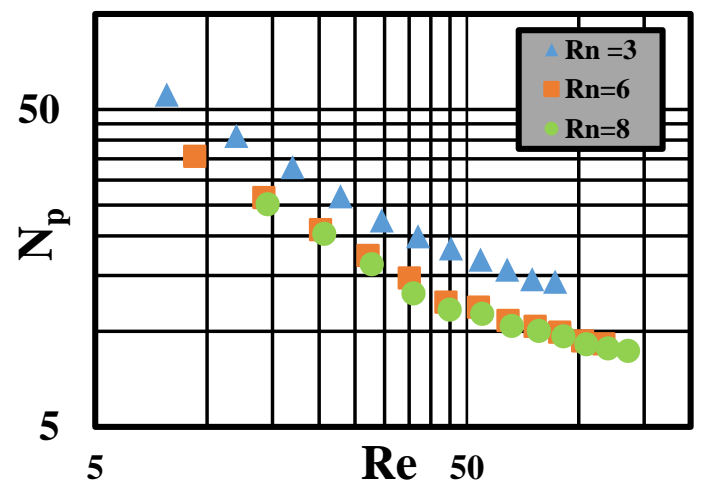

Figure (5.2-3). Power curves for the double Scaba-anchor coaxial mixer at different speed ratios in the co-rotating mode and $1 \%$ xanthan gum solution obtained using (a) Pakzad et al. model and (b) Bao et al. model. 
The power curves were also generated for different xanthan gum concentrations $(1.0 \%$, $1.5 \%$, and $2 \%$ ) at the speed ratio of $R_{n}=8$ in the co-rotating mode as shown in Figures $(5.2-4)$.

(a)

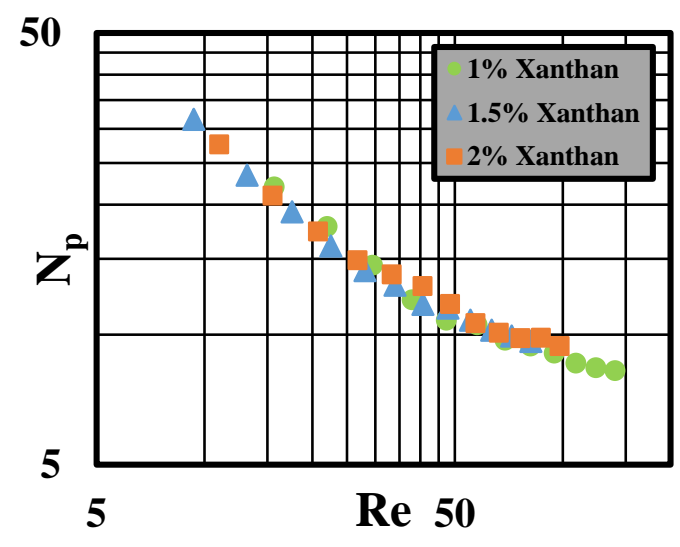

(b)

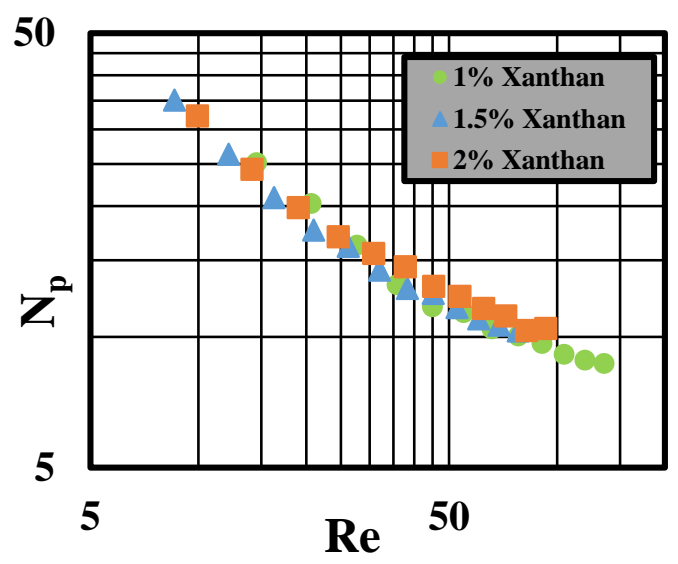

Figure (5.2-4). Power curves for the double Scaba-anchor coaxial mixer at different xanthan gum solutions in the co-rotating mode at the speed ratio $R_{n}=8$ in co-rotating mode for: (a) Pakzad model and (b) Bao model.

These results demonstrate that the power curves generated at various concentrations using both models collapsed to one curve at the speed ratio of $R_{n}=8$. Thus, it can be concluded that the correlations proposed by Pakzad et al. (2013 b) can be applied to achieve the power curve of the double Scaba-anchor system at different Reynold numbers for a wide range of the speed ratios. However, the correlations proposed by Bao et al. (2011) can be used only at the speed ratios higher than 5 .

\subsubsection{Flow Pattern}

The mixing efficiency of a stirred system is affected by the flow pattern, which depends on the factors such as the speed ratio (rotational speed of the central impeller/rotational 
speed of the anchor impeller), the geometry of the impeller, the number of the impellers, impeller spacing, location of the impeller in the vessel, the presence of baffle or other internals, and rheology of the fluid [Chhabara and Richardson, 1999). The focus of this section is to evaluate both numerically and experimentally, the mixing performances of the single Scaba-anchor coaxial (SSAC) and the double Scaba-anchor coaxial (DSAC) mixers by comparing the flow pattern generated inside the mixing vessel at the fixed power consumption.

Electrical resistance tomography (ERT) as a non-intrusive visualization method was utilized to evaluate the mixing quality. The 2D and 3D tomography images are depicted in Figure (5.2-5). These images show the conductivity distributions within the agitated vessels equipped by the SSAC and DSAC mixers for $1.0 \%$ xanthan gum solution at $R_{n}=$ 8 and $P=190.5 \mathrm{~W} / \mathrm{m}^{3}$ in the co-rotating mode. After $20 \mathrm{~s}$ following the injection of 50 $\mathrm{mL}$ salt solution on Plane 1, the conductivity distributions were monitored for both the SSAC [Figure (5.2-5)a] and the DSAC [Figure (5.2-5) c] mixers. It can be seen that the tracer was dispersed and pushed towards the lower planes by the upper Scaba impeller of the DSAC mixer. However, the dispersion of the tracer by the SSAC mixer in the axial direction was not as effective as that attained by the DSAC mixer. Furthermore, [Figures (5.2-5) $\mathrm{b}$ and (5.2-5) d] illustrate the conductivity distributions after $317 \mathrm{~s}$ following the tracer injection inside the tanks equipped with the SSAC and DSAC mixers, respectively. These images demonstrate that the degree of homogeneity achieved by the DSAC mixer was better than that achieved by the SSAC mixer at the same power consumption. 
(a)

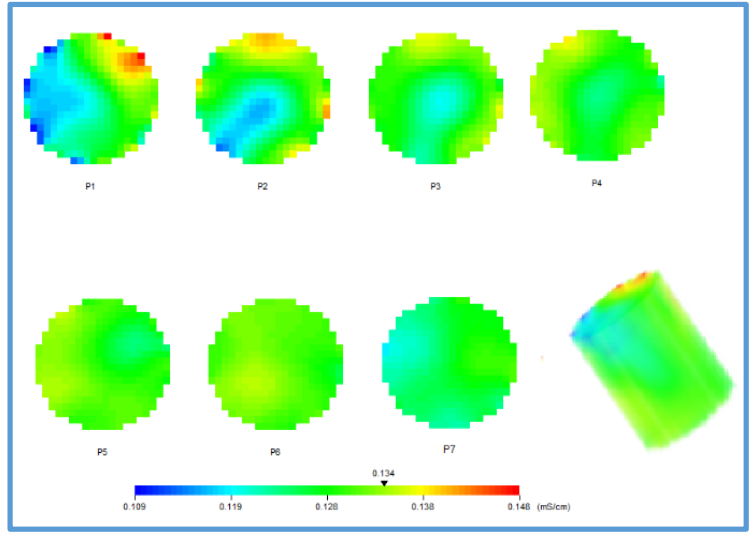

(c)

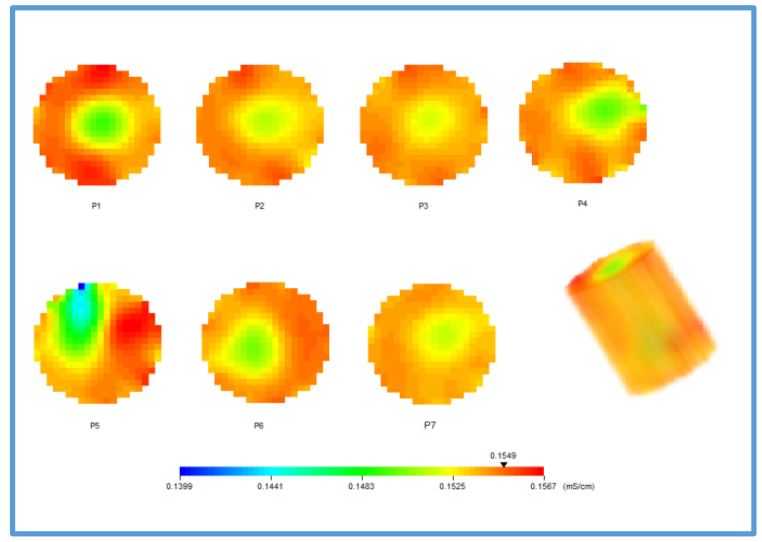

(b)

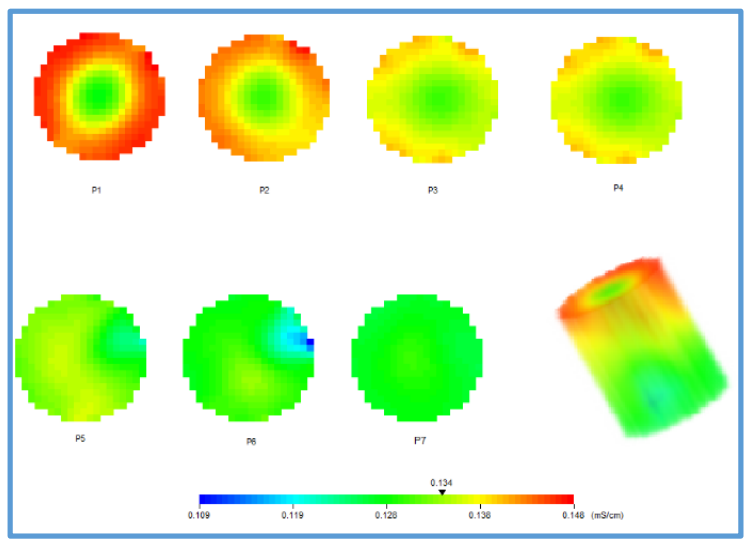

(d)

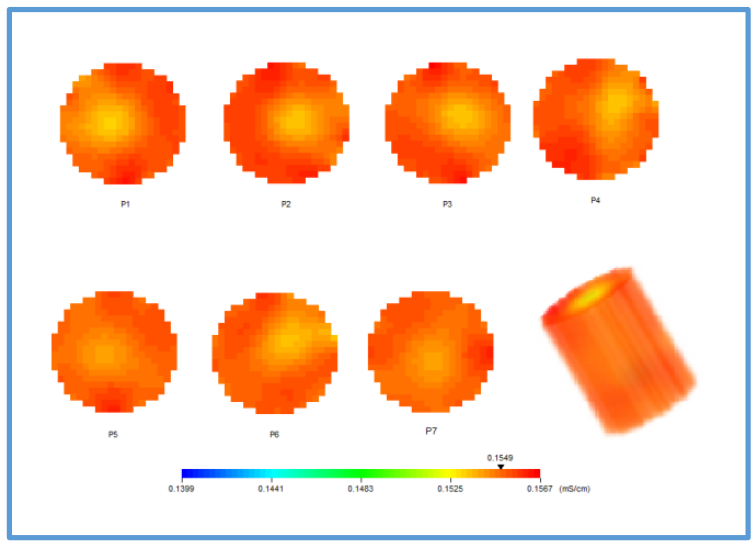

Figure (5.2-5). 2D and 3D tomography images at $P=190.5 \mathrm{~W} / \mathrm{m}^{3}, R_{n}=8$, and $1 \%$ xanthan gum solution in the co-rotating mode for: (a) SSAC mixer at $t=20 \mathrm{~s}$, (b) DSAC mixer at $t=20 \mathrm{~s}$, (c) SSAC mixer at $t=317 \mathrm{~s}$, and (d) DSAC mixer at $t=317 \mathrm{~s}$.

To understand and analyze the flow field generated by the coaxial mixers inside the stirred tank, the velocity contour plots obtained from the CFD model for the vertical and horizontal planes are depicted in Figure (5.2-6). As it can be noticed in Figure (5.2-6) a, the use of the single Scaba-anchor coaxial mixer (SSAC) resulted in the formation of a small cavern, well-mixed region, with the higher fluid velocities around the Scaba impeller while a large dead zone was formed in the upper part of the tank and near the 
fluid surface. However, as shown in Figure (5.2-6) b, when the double Scaba-anchor coaxial mixer (DSAC) was employed with the same power consumption as the single Scaba-anchor coaxial mixer (SSAC), fluid flow was intensified in both axial and radial directions leading to the elimination of the dead regions in the upper part of the mixing vessel. The velocity contour plots for the central impeller plane in Figure (5.2-6) shows that the fluid velocity around the central impeller was higher for the SAC compared to that for the DSAC. However, the vertical contour plots demonstrates more uniform flow and less dead zones inside the tank equipped with the DSAC compared to that for SSAC at the same power consumption. 
(a)
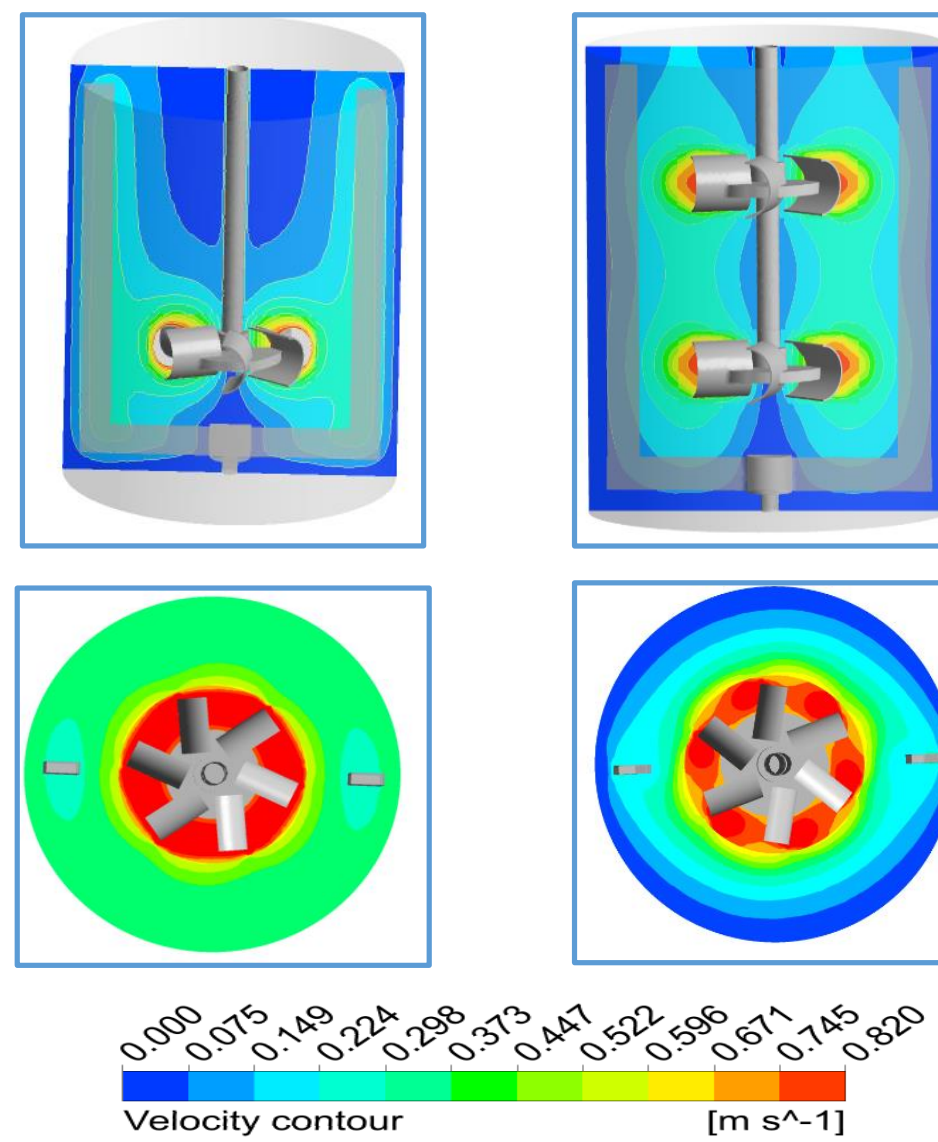

Figure (5.2-6). Vertical and horizontal velocity $(\mathrm{m} / \mathrm{s})$ contour plots at $P=190.5 \mathrm{~W} / \mathrm{m}, R_{n}$ $=8$, and $1 \%$ xanthan gum solution in the co-rotating mode for: (a) Single Scaba-anchor coaxial (SSAC) mixer and (b) Double Scaba-anchor coaxial (DSAC) mixer.

For more in-depth analysis, the vertical and horizontal velocity vector plots for the SSAC and DSAC coaxial mixers at the fixed power consumption of $P=190.5 \mathrm{~W} / \mathrm{m}^{3}$ are shown in Figure (5.2-7). As it can be noticed in Figure (5.2-7) a fluid flow created by the SSAC mixer was mainly occurred inside the torus (Rivera et al., 2006) formed around the Scaba impeller in the lower part of the tank. The fluid was discharged directly towards the vessel wall by the Scaba impeller and then it was divided upward and downward producing two main circulation loops above and below the central Scaba impeller. In 
fact, the fluid was returned to the impeller by these two circulating loops. As it can be seen, the single Scaba impeller was not able to generate adequate axial flow in the upper part of the tank between the anchor and the tank wall. The flow in the upper part of the tank was mostly produced by the anchor impeller close to the tank wall and the stagnant and/or slow moving fluid areas occurred near the shaft and under the fluid surface. However, for a DSAC coaxial mixer, as shown in Figure (5.2-7) b, four circulation loops were created around two central impellers. In fact, a stable parallel flow (Rutherford et al., 1996) was achieved and resulted in the adequate circulation inside the tank. This flow pattern intensified the axial flow leading to a continuous motion of the flow from the lower part to the upper part of the tank particularly between anchor and the tank wall. As a consequence, mixing achieved by using the DSAC mixer was more efficient than that for the SSAC mixer. This comparison will be quantified later in this paper with regard to the power drawn and mixing time. 
(a)
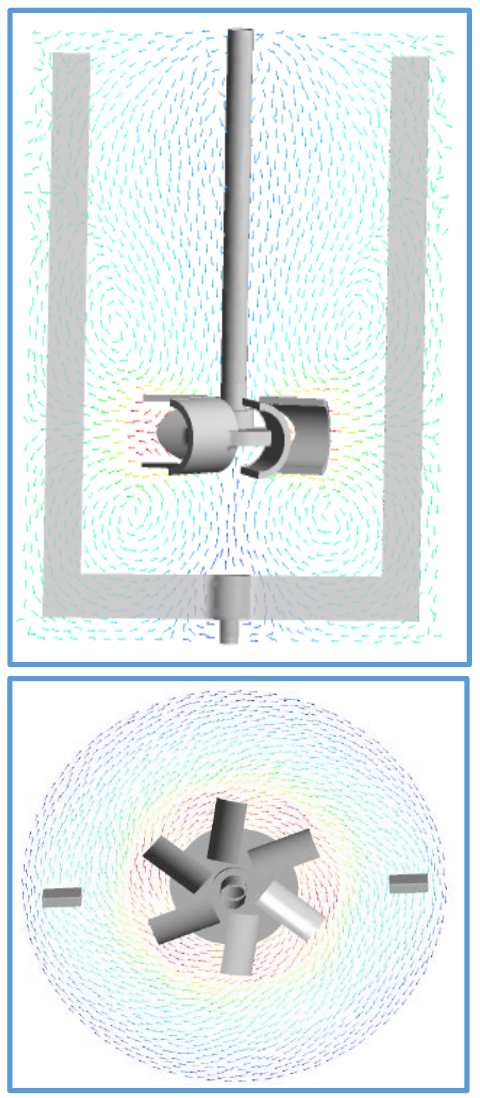

(b)
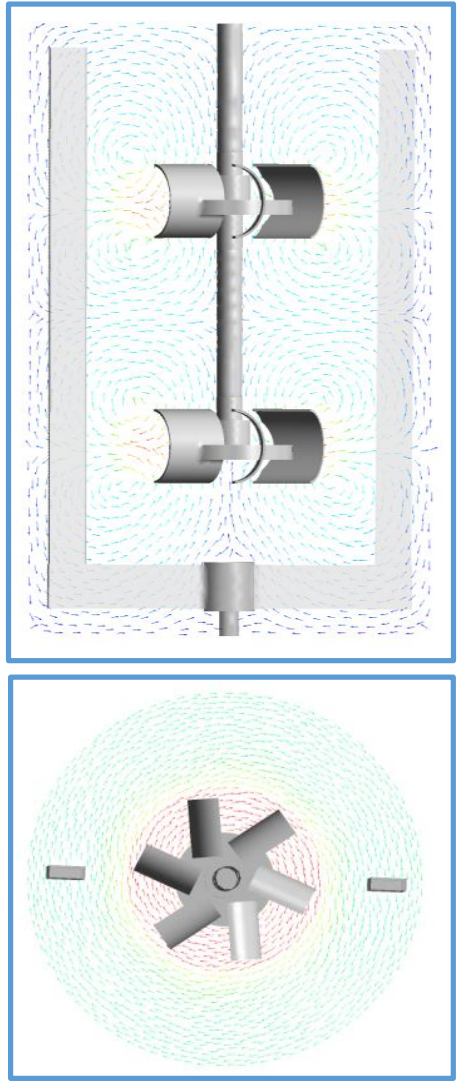

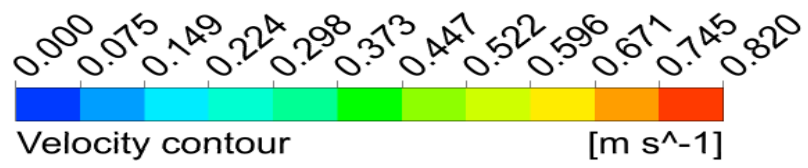

Figure (5.2-7). Vertical and horizontal velocity (m/s) vector plots at $P=190.5 \mathrm{~W} / \mathrm{m}^{3}, R_{n}$ $=8$, and $1 \%$ xanthan gum solution in the co-rotating mode for: (a) Single Scaba-anchor coaxial (SSAC) mixer and (b) Double Scaba-anchor coaxial (DSAC) mixer.

To quantitatively compare the mixing performances of the SSAC and DSAC coaxial mixers in the co-rotating mode, the dimensionless axial, radial and tangential velocity profiles were calculated along the tank height using the CFD model as shown in Figure (5.2-8). Firstly, these data clearly depict the fact that the maximum dimensionless axial 
velocity achieved by the SSAC coaxial mixer with one central impeller was $V_{a}=$ $0.0142 V_{t i p}$, which was higher than that for the DSAC coaxial mixer with double central impellers. The higher axial velocities for the SSCA mixer was mostly generated close to the central impeller with significant decrease of the axial velocity in the upper part of the vessel form $Z / H=0.7$ to $Z / H=1.0$. It must be mentioned that the rotational speed of the central impeller of the SSAC mixer was higher than that for the DSAC mixer to achieve the same power consumption of $190.5 \mathrm{~W} / \mathrm{m}^{3}$ for both coaxial configurations. In contrast, the maximum axial velocity achieved by the DSAC mixer consisting of two central impeller was less than that for the SSAC mixer comprising of one central impeller. However, the axial velocity profile generated by the DSAC mixer was more uniform inside the mixing tank from the lower part to the upper part of the vessel (from $Z / H=0.0$ to $Z / H=1.0)$ compared to the SSAC mixer.

Secondly, Figure (5.2-8) b shows that both SAC and DSAC coaxial mixers generated the higher radial velocities close to the central impellers, however the radial velocity profile generated by the DSAC mixer was more consistent than that by the SSAC mixer along the tank height. In fact, a low radial velocity region was formed in the upper part of the vessel from $Z / H=0.7$ to $Z / H=1.0$ when the SSAC coaxial mixer with only one central impeller was utilized. Finally, it can be observed from the comparison of the dimensionless tangential velocity profiles for both coaxial mixers [Figure (5.2-8) c] that the SSAC coaxial mixer generated a higher tangential velocity than that of the DSAC coaxial mixer. However, the distribution of the tangential velocity for DSAC was more consistent than that for SSAC mixer. Moreover, it was noticed that the generated tangential velocities were clockwise (negative sign) for both SAC and DSAC in co- 
rotating mode. As a result, the flow segregation was not noticeable for both agitated systems in co-rotating mode (Rivera et al., 2006).

(a)

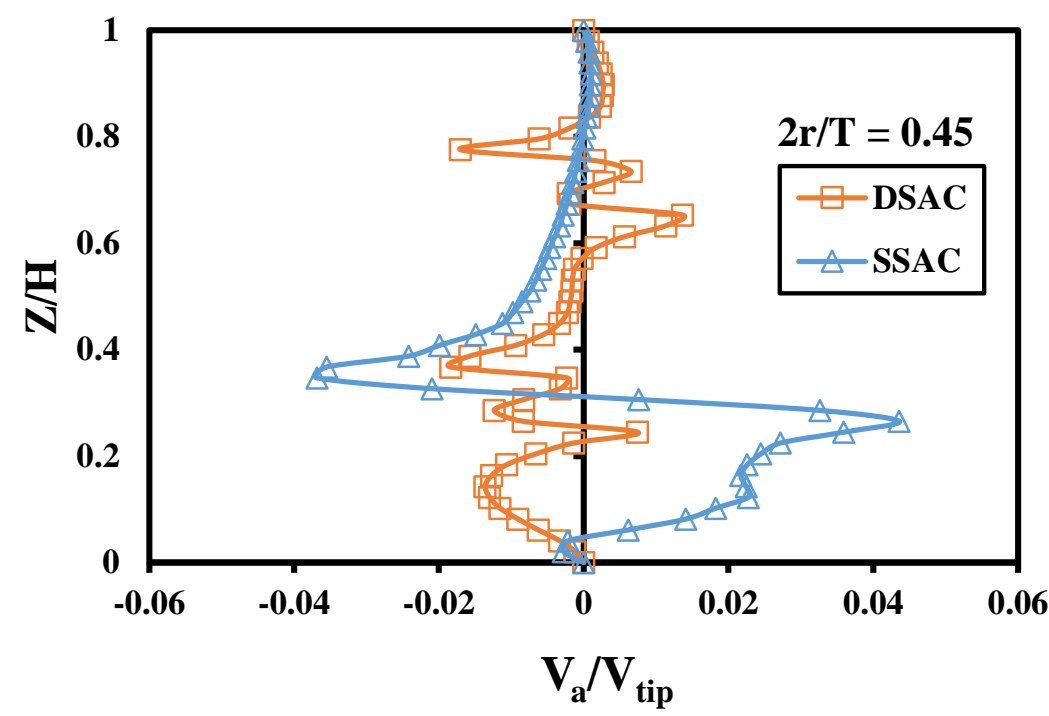

(b)

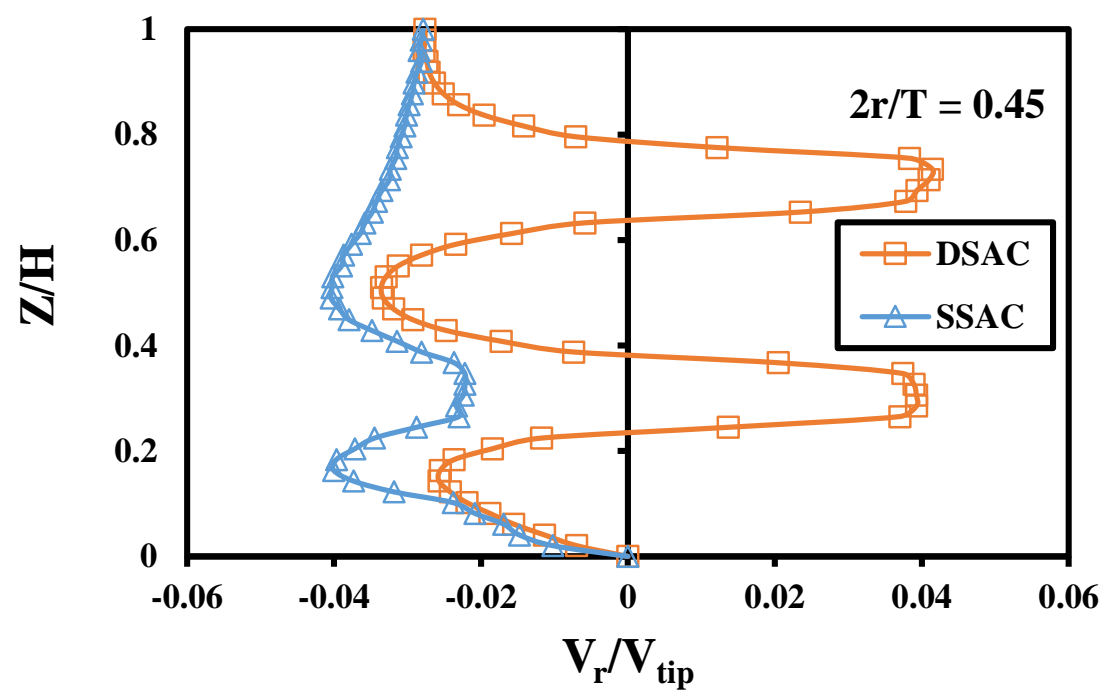


(c)

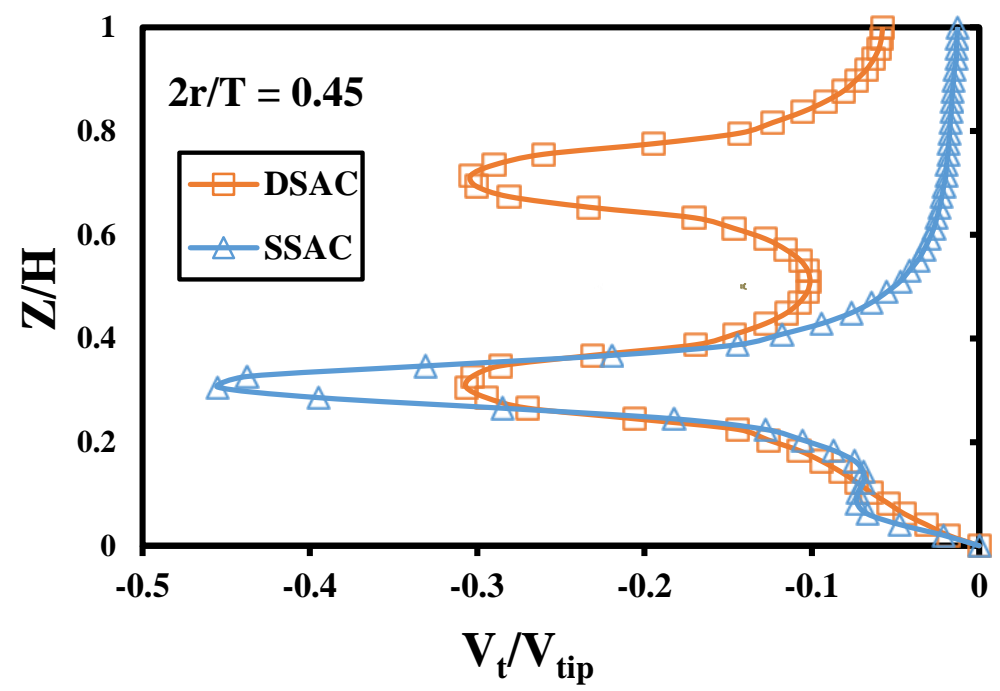

Figure (5.2-8). Dimensionless axial velocity profiles along the tank height for both single Scaba-anchor coaxial (SSAC) with Scaba $\left(Z_{1} / H\right)=0.34$ and double Scaba-anchor coaxial (DSAC) mixers with two Scaba $\left(\mathrm{Z}_{1} / \mathrm{H}=0.34\right.$ and $\left.\mathrm{Z}_{2} / \mathrm{H}=0.72\right)$ at $2 r / D=0.45$, from $y=-$ 0.09 to $y=+0.09, P=190.5 \mathrm{~W} / \mathrm{m}^{3}, R_{n}=8$, and $1 \%$ xanthan gum solution in the corotating mode for: (a) axial velocity, (b) radial velocity, and (c) tangential velocity.

For a better analysis of the flow field created by the coaxial mixers, the average shear strain rates were plotted along the tank height at different radial positions for both configurations and the results are shown in Figure (5.2-9). It can be noticed that the deformation profiles generated by two coaxial systems were reasonably different. For the SSAC coaxial mixer [Figure (5.2-9) a] a larger deformation (higher shear rate) was attained in the area close to the Scaba impeller. It was started from an almost zero value in the lower part of the tank with an increase to the tip of impeller $\left(\dot{\gamma} / N_{C o}=0.4\right)$ and then it was reversed in the upper part of the tank. This generated a small cavern, well-mixed region, around the Scaba impeller and the slow moving fluid and/or dead zones at the bottom and upper part of the tank and below the liquid surface. However, for the DSAC coaxial mixer, as depicted in Figure (5.2-9)b, it can be seen that for the lower impeller the 
average shear rate started from a near zero value close to the bottom of the tank with an increase to the tip of the impeller $\left(\dot{\gamma} / N_{C O}=25\right)$, and then it was reversed above the impeller. The same trend can be observed for the upper impeller as well. In fact, the distribution of the rate of deformation from the lower to the upper part of the vessel was more uniform for the DSAC than that for the SSAC mixer. Thus, the size of the stagnant and/or slow moving fluid regions observed inside the tank equipped with the SSAC mixer was reduced when the DSAC mixer with the same power consumption was utilized.

(a)

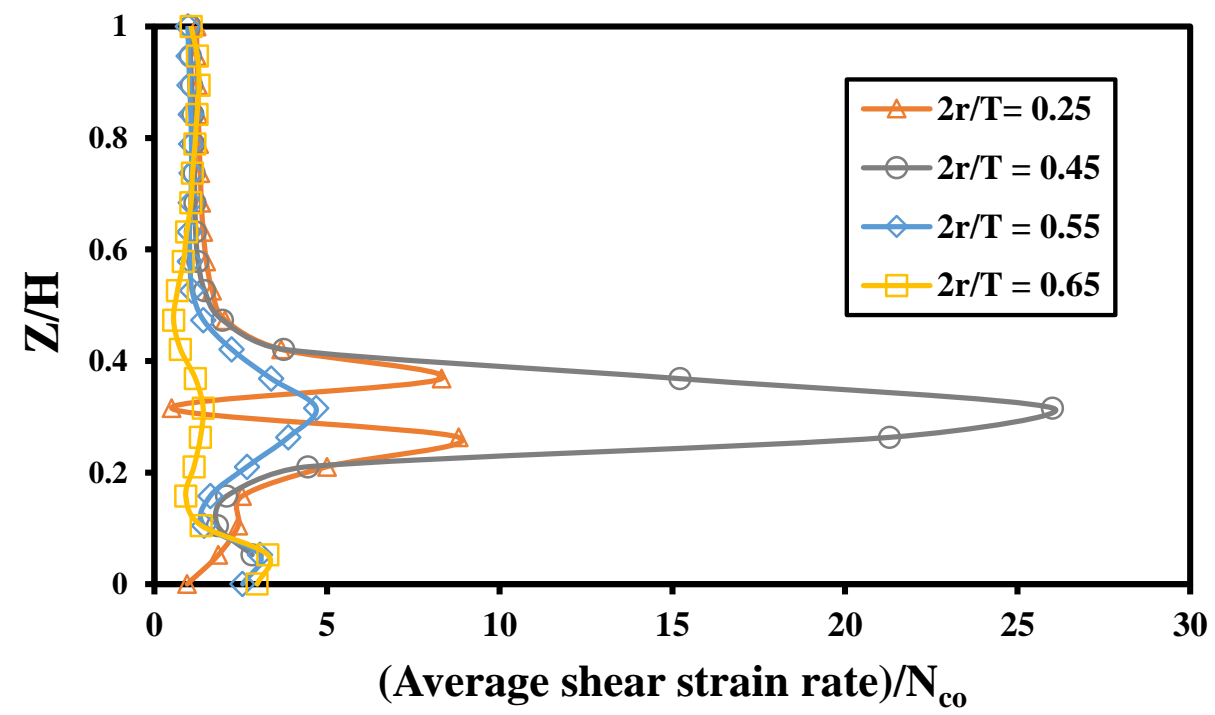


(b)

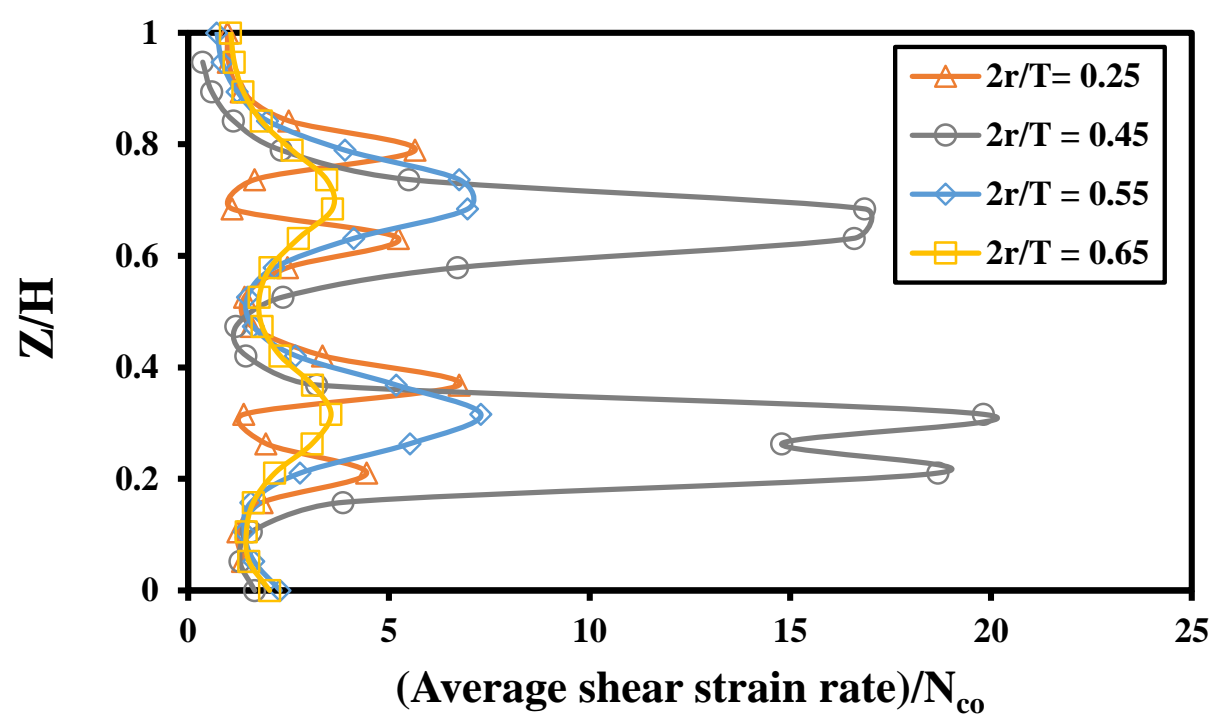

Figure (5.2-9). Dimensionless average shear strain rate along the tank height for both single Scaba-anchor coaxial (SSAC) with Scaba $\left(Z_{l} / H=0.34\right)$ and double Scaba-anchor coaxial $(D S A C)$ mixers with two scaba $\left(Z_{1} / H=0.34\right.$ and $\left.Z_{2} / H=0.72\right)$ at different radial positions $(2 r / D=0.25,0.45,0.55$, and 0.65$), P=190.5 \mathrm{~W} / \mathrm{m}^{3}, R_{n}=8$, and $1 \%$ xanthan gum solution in the co-rotating mode for: (a) single Scaba-anchor coaxial (SSAC) mixer and (b) double Scaba-anchor coaxial (DSAC) mixer.

The CFD model was also utilized to obtain the viscosity contour plots, both horizontally and vertically, for both SSAC and DSAC mixers and the results are shown in Figure (5.210). As mentioned earlier, the apparent viscosity of the pseudoplastic fluids depends on the shear rate at any point inside the mixing vessel. It can be noticed from Figure (5.2-10) a that for the SSAC mixer a low-viscosity region around the central impeller (higher shear rate) was surrounded by the high-viscosity zone in the rest of the tank especially in the upper part of the vessel. The existence of these high-viscosity zones generally reduces the mixing quality inside the mixing system. However, Figure (5.2-10) b demonstrates that the use of the DSAC configuration significantly decreased the formation of the high- 
viscosity regions. In fact, the use of two central impellers resulted in a more uniform distribution of the shear rate throughout the tank. It must be mentioned that the results presented in Figure (5.2-10) for both SSAC and DSAC mixers were achieved at the same power consumption.

(a)
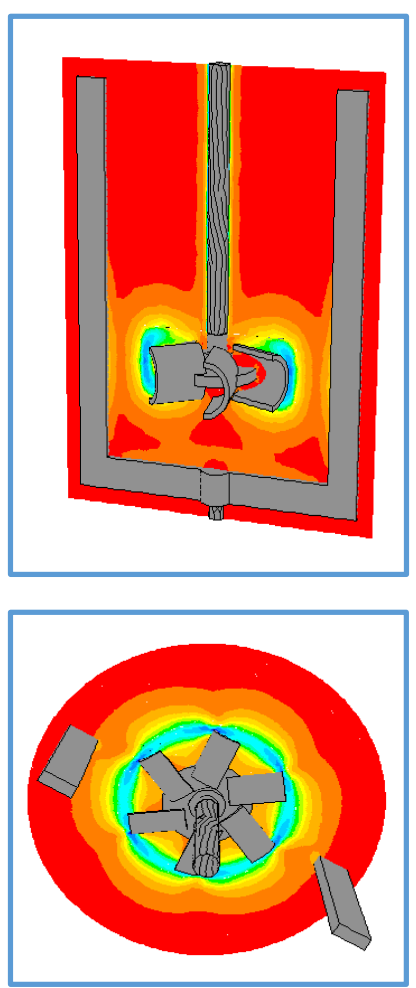

(b)
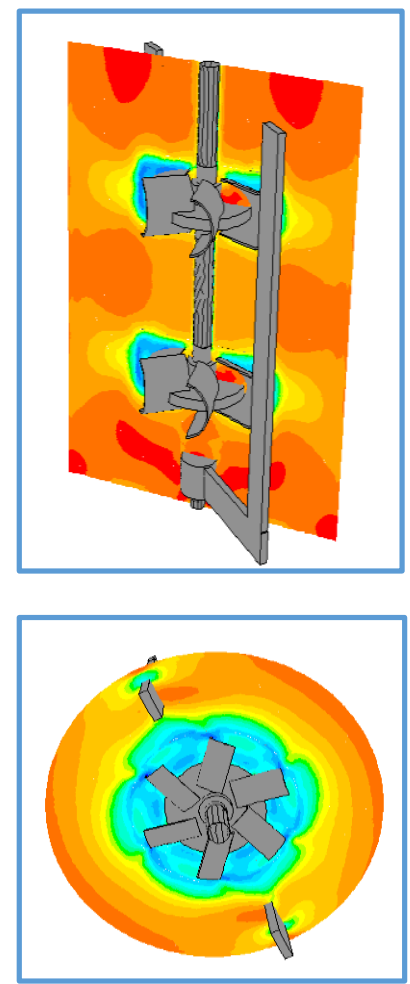

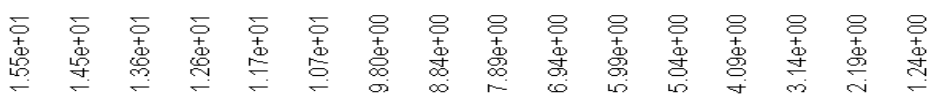

Figure (5.2-10). Vertical and horizontal viscosity $(\mathrm{Kg} / \mathrm{m} \mathrm{s})$ contour plots at $P=190.5$ $\mathrm{W} / \mathrm{m}^{3}, R_{n}=8$, and $1 \%$ xanthan gum solution in the co-rotating mode for: (a) single Scaba-anchor coaxial (SSAC) mixer and (b) double Scaba-anchor coaxial (DSAC) mixer. 


\subsubsection{Mixing Time}

To compare the performances of the SSAC and DSAC coaxial mixers in the mixing of the yield-pseudoplastic fluids in the co-rotating mode, the mixing time values against the power consumption are presented in Figure (5.2-11) for the speed ratios of 3, 6, and 8 . These data clearly illustrate that the blending times were decreased with an increase in power consumption at different speed ratios for both coaxial mixers. However, for a fixed power consumption, the mixing times attained by the DSAC mixer were shorter than those by the SSAC mixer at all speed ratios. This finding is also supported by the information provided in the previous sections of this paper regarding the velocity profiles, average shear strain rates, and apparent viscosity contours for the two coaxial mixing configurations. In fact, the results presented in this paper show that the flow domain generated by the DSAC mixer was more efficient in reducing the slow moving fluid and/or the stagnant regions inside the mixing vessel than that by the SSAC mixer.

(a)

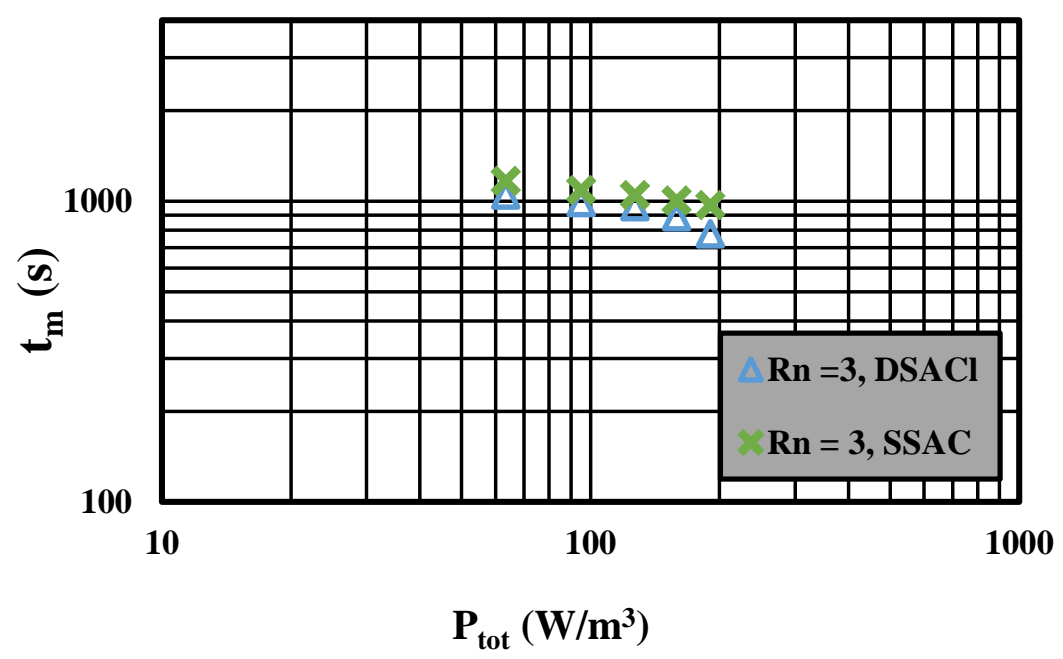


(b)

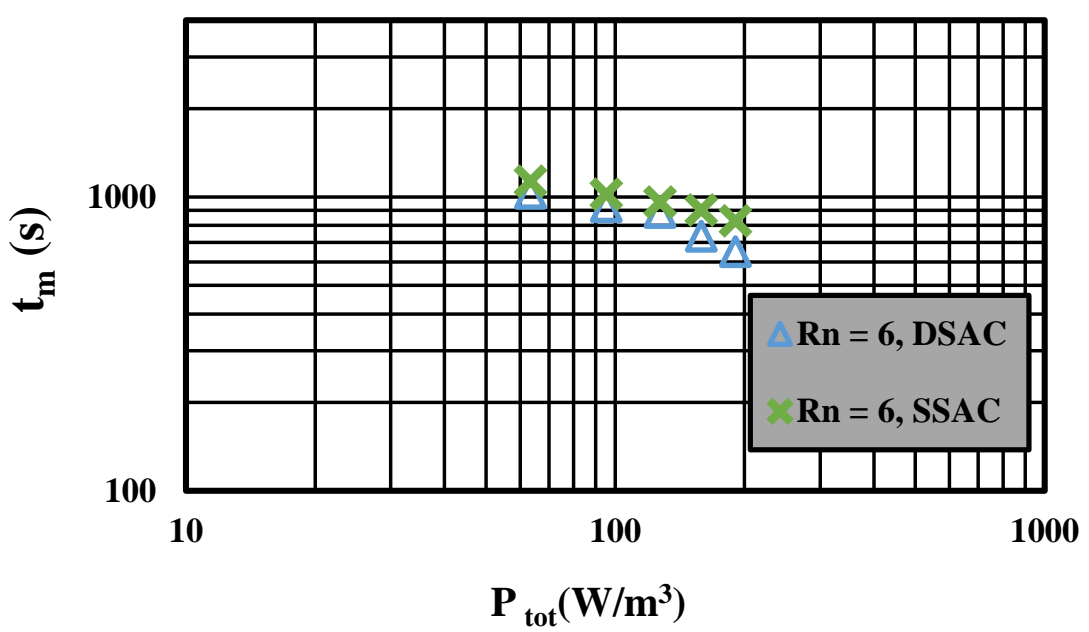

(c)

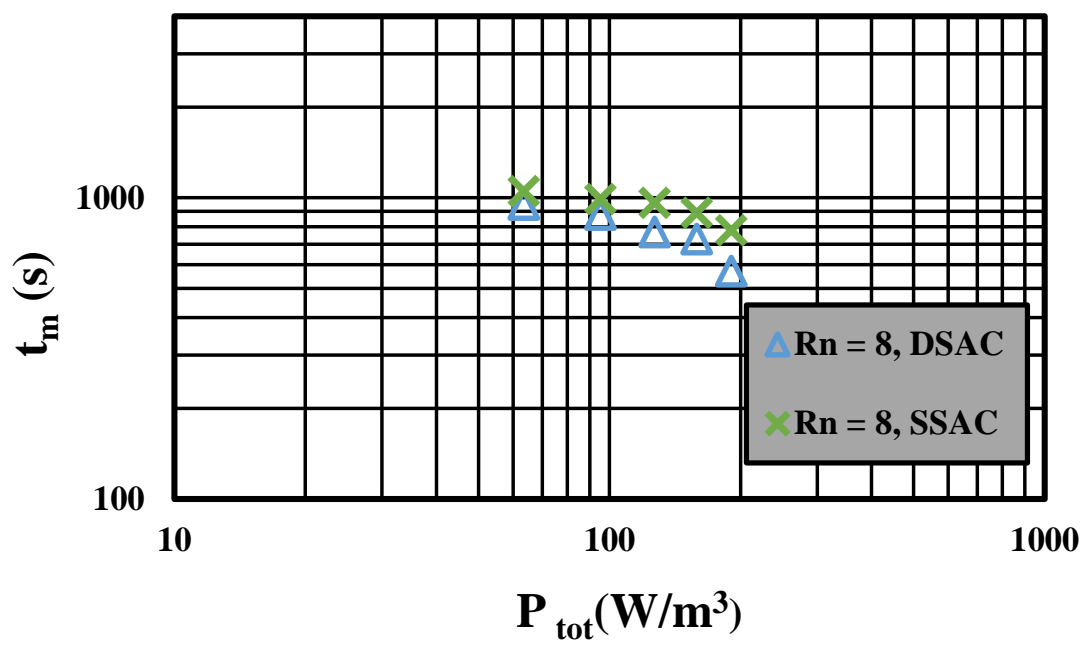

Figure (5.2-11). Mixing time versus total specific power consumption for $1 \%$ xanthan gum solution for both single Scaba-anchor coaxial (SSAC) and double Scaba-anchor coaxial (DSAC) mixers in the co-rotating mode: (a) $R_{n}=3$, (b) $R_{n}=6$, and (c) $R_{n}=8$.

\subsubsection{Mixing Efficiency}

To better evaluate the mixing efficiencies of the coaxial mixers utilized in this work, the mixing rate $\left(\pi_{3}\right)$ and the mixing time Reynolds $\left(R e_{m}=1 / \pi_{2}\right)$ proposed by Zlokarnic $(2001)$ were employed to determine the optimum conditions for the mixing process at the 
minimum mixing work (min P.t $t_{m}$ ). The dimensionless numbers $\pi_{3}$ and $\pi_{2}$ are defined as follows:

$$
\begin{array}{r}
\operatorname{Re}_{\mathrm{m}}=\frac{1}{\pi 2}=\frac{T^{2} \rho}{t_{m} \eta} \\
\pi_{3}=\frac{P_{t o t} t_{m}{ }^{2}}{T^{3} \eta}
\end{array}
$$

Here $t_{m}, \eta, P_{t o t}$, and $T$ represent mixing time, apparent viscosity of non-Newtonian fluid, total power consumption, and the tank diameter.

To determine the efficient coaxial mixer, the mixing energy $\left(\pi_{3}\right)$ versus the mixing time Reynolds number $\left(R e_{\mathrm{m}}\right)$ was plotted at the speed ratio of $R n=8$ for both coaxial mixers based on Equations (5.2-7) and (5.2-8) as shown in Figure (5.2-12). These data clearly demonstrate that the mixing energy decreased with an increase of $R e_{m}$ for both SSAC and DSAC mixers. However, at a fixed mixing time Reynolds number, the mixing energy for the DSAC mixer was reasonably less than that for the SSAC mixer. These results confirm our previous finding that the DSAC mixer was more effective than the SSAC mixer in the mixing of the yield-pseudoplastic fluids. 


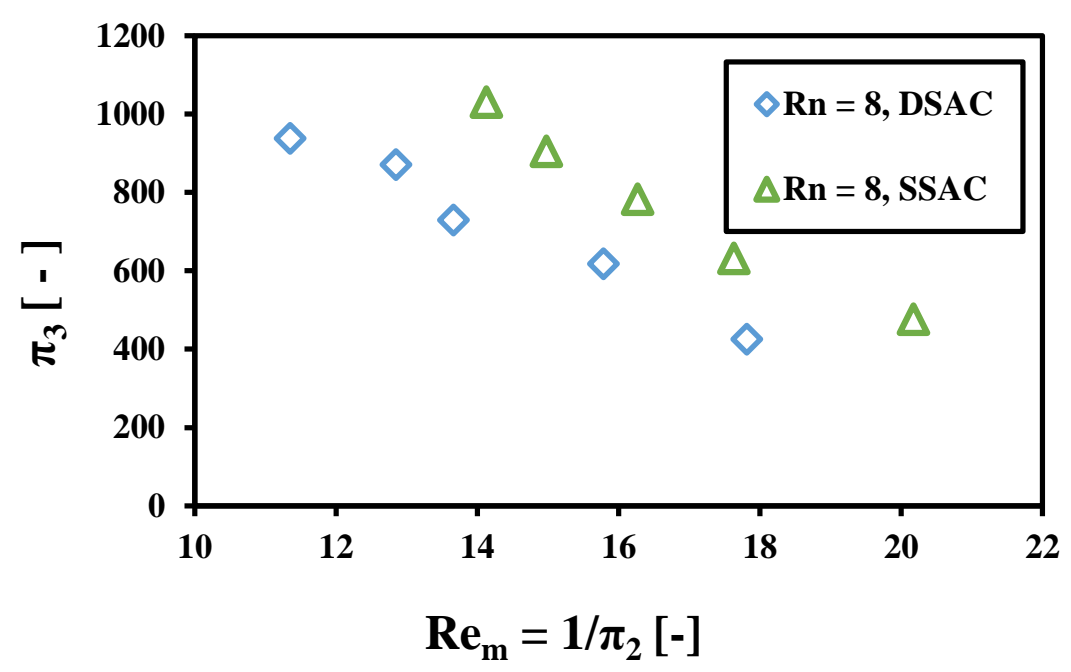

Figure (5.2-12) Mixing energy versus mixing time $R e_{m}$ number at $R_{n}=8$ and $1 \%$ xanthan gum solution in the co-rotating mode for both single Scaba-anchor coaxial (SSAC) and double Scaba-anchor coaxial (DSAC) mixers.

\subsubsection{Conclusion}

The hydrodynamic performance of the DSAC coaxial mixer (two Scaba impellers in combination with an anchor impeller) was assessed and compared to that of the SSAC coaxial mixer (single Scabe impeller in combination with an anchor impeller) with regards to the power consumption, mixing time, generated flow domain, and mixing efficiency by using the electrical resistance tomography (ERT) and computational fluid dynamics (CFD). To plot the power curve (power number as a function of Reynolds number) for the DSAC coaxial mixer, various correlations proposed for the generalized power and Reynolds numbers in the literature for the coaxial mixers were evaluated. Our results show that all curves generated by Pakzad et al. model (2013b) at different speed ratios collapsed to one curve in the co-rotating mode. In contrast, the power plots generated using Bao et al. model (2011) collapsed to one curve at the higher speed ratios while the power curves did not coincide reasonably at the lower speed ratios. 
The simulated flow domain through the validated CFD model revealed that the use of the single Scaba-anchor coaxial mixer (SSAC) resulted in the formation of a small cavern, well-mixed region, with the higher fluid velocities around the Scaba impeller while a large dead zone was formed in the upper part of the vessel and near the fluid surface. However, when the double Scaba-anchor coaxial mixer (DSAC) was employed with the same power consumption as the single Scaba-anchor coaxial mixer (SSAC), fluid flow was intensified in both axial and radial directions leading to the elimination of the dead regions in the upper part of the mixing vessel. Overall, the use of two central impellers resulted in a more uniform distribution of the shear strain rate throughout the stirred vessel. To quantify the efficiencies of the DSAC and SSAC coaxial mixers, the mixing energy versus the mixing time Reynolds number was plotted for both coaxial mixers. These results indicated that the DSAC coaxial stirrer was more effective than the SSAC stirrer in the mixing of the yield-pseudoplastic fluids. 


\subsection{A New Perspective in the Evaluation of the Mixing of Biopolymer Solutions with Different Coaxial Mixers Comprising of Two Dispersing Impellers and a Wall Scraping Anchor}

\subsubsection{Introduction}

The goal of this research work was to analyze the hydrodynamic performance of the double central impeller-anchor mixer in the co-rotating mode when the fluid flow generated inside the tank was in the laminar or transitional regime. Three different coaxial mixers, namely, double Scaba-anchor coaxial (DSAC), double Rushton turbineanchor coaxial (DRAC), and double pitched blade turbine-anchor coaxial (DPAC) mixers [Figure (5.3-1)], were utilized in this work. To fulfill the objectives of this work, three techniques were adopted: electrical resistance tomography (ERT) to measure the degree of homogeneity and visualize inside of the mixing vessel, the computational fluid dynamics (CFD) to simulate the flow domain of the fluid, and design of experiments (DOE) combined with the response surface methodology (RSM) to analyze the mixing data. The hydrodynamic performances of the investigated coaxial agitated mixers were assessed and compared directly with respect to the mixing times, power input and flow characterization both numerically and experimentally. The performances of these coaxial mixers were assessed at the operating conditions listed in Table (5.3-1). 

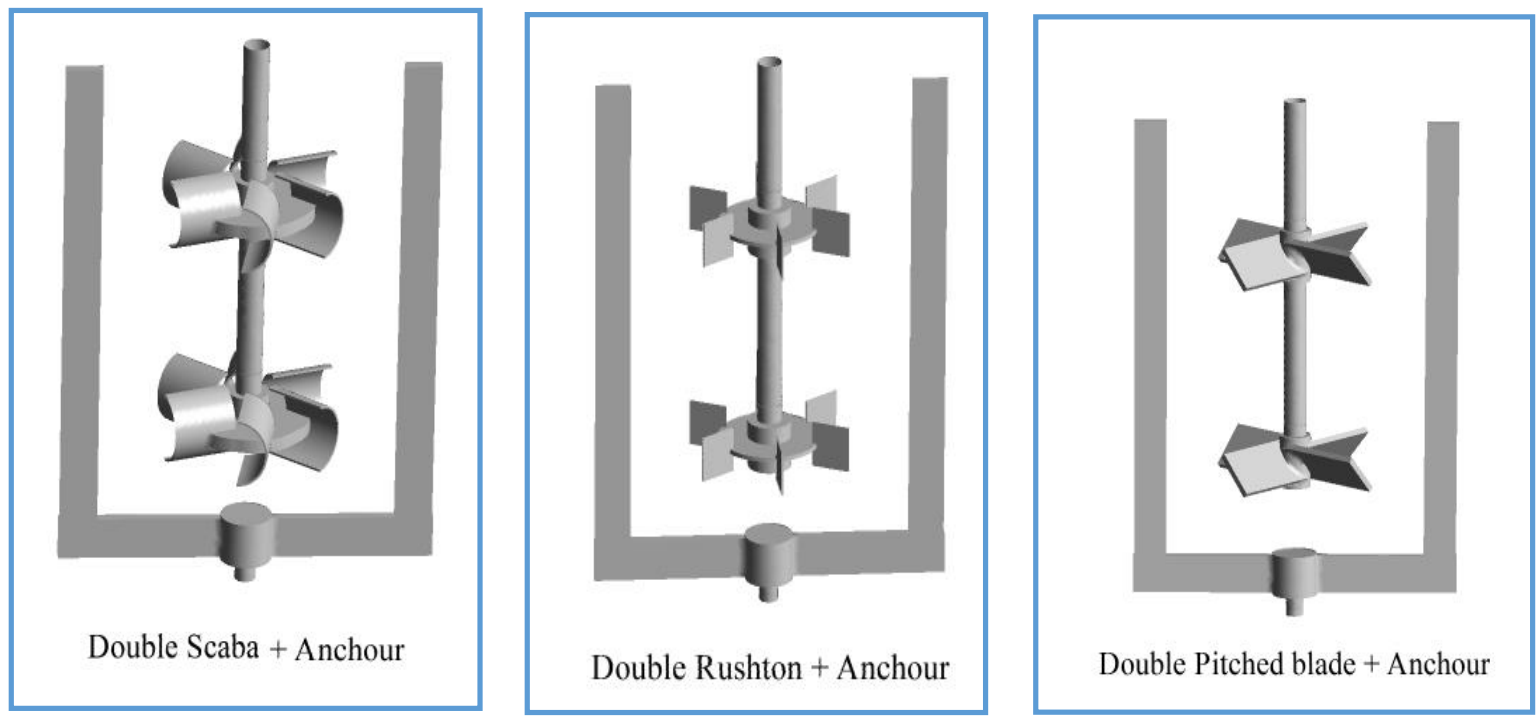

Figure (5.3-1). Schematic diagram of the experimental set-up and different coaxial mixer configurations analyzed in this study.

Table (5.3-1). Operating conditions employed in this study.

\begin{tabular}{lc}
\hline Description & Range and Type \\
\hline Central impeller types & Scaba, Rushton turbines, Pitched-blade \\
& turbines with $45^{\circ}$ \\
Central impeller speed $\left(N_{c}\right)$ & $0-300(\mathrm{rpm})$ \\
Anchor impeller speed $\left(N_{a}\right)$ & $25-35(\mathrm{rpm})$ \\
Impeller speed ratio $\left(N_{c} / N_{a}\right)$ & $4,5,6,8,10$ \\
Central Impeller Diameter $\left(D_{c}\right)$ & $0.18 \mathrm{~m}$ \\
Impeller spacing & $T / 2=0.40 \mathrm{H}$ \\
Impeller off-bottom & $0.30 \mathrm{H}$ \\
Submerge surface & $0.30 \mathrm{H}$ \\
Xanthan gum concentration $(\%)$ & $1,1.5,2$ \\
\hline
\end{tabular}


The Box-Behnken experimental design (Box and Behnken, 1960) and the response surface methodology (RSM) (Myers and Montgomery, 2002) were used to explore and determine the influence of the independent variables (inputs) on the outcome of the mixing operation. The Box-Behnken experimental design (BBD) requires three levels of each variable which are coded as $-1,0$, and 1 . In the present study, the mixing efficiency of the coaxial mixer was evaluated with respect to the mixing time and specific power input by means of a four-factor and three-level BBD in combination with the RSM. The independent variables listed in Table (5.3-2) were coaxial configuration $\left(X_{1}\right)$, xanthan gum solution $\left(X_{2}\right)$, central impeller speed $\left(X_{3}\right)$, and anchor speed impeller $\left(X_{4}\right)$. The range of each factor was selected based on the preliminary study.

Table (5.3-2). Experimental range and levels of the independent variables.

\begin{tabular}{lcccc}
\hline \multicolumn{1}{c}{ Variables } & Symbol & -1 & 0 & 1 \\
\hline Coaxial configuration (-) & $X_{1}$ & DPAC & DSAC & DRAC \\
Xanthan gum concentration & $X_{2}$ & 1.0 & 1.5 & 2.0 \\
Central impeller speed (rpm) & $X_{3}$ & 150 & 195 & 240 \\
Anchor impeller speed (rpm) & $X_{4}$ & 25 & 30 & 35 \\
\hline
\end{tabular}

Experimental data generated by BBD design can be fitted to the following quadratic equation model (second order polynomial) by the least-squares regression using version 9 of Design-Expert software:

$$
Y=\beta_{0}+\sum_{i=1}^{k} \beta_{i} X_{i}+\sum_{i=1}^{j-1} \sum_{j=1}^{k} \beta_{i j} X_{i} X_{j}+\sum_{i=1}^{k} \beta_{i i} X_{j i}^{2}+e_{i}
$$


where $Y, X_{i} X_{j}, \beta_{0}, \beta_{i}, \beta_{i j}, \beta_{i i}, k$, and $e_{i}$ represent the predicted response, independent variables, constant coefficient, the influence of independent variable, the influence of interaction among variables, quadratic effect, the number of variables, and error residual, respectively.

Analysis of variance (ANOVA) as a powerful statistical technique was used to find the statistical significance of the model equation. To validate the model, actual data were compared to the predicted data. Then the effect of independent variables on the hydrodynamic performance of the three different configurations of the coaxial mixers were analyzed by developing three dimensional (3-D) and two dimensional (2-D) contour plots.

The numerical model for simulations was developed by CFD. The experimental measurements of the mixing time and torque for $1 \%$ xanthan gum solution at different operating conditions were compared with the CFD results to validate the applicability of the CFD model. The computed data showed a very good agreement (the maximum standard deviation $<5 \%$ ) with the experimental data as shown in Table (5.3-3). The validated model was then utilized to obtain further information for the assessment of the investigated coaxial mixers in this study. 
Table (5.3-3). Computed and measured torque and mixing time for $1 \%$ xanthan gum solution agitated at $R_{n}=8$ by Scaba-anchor coaxial mixer.

\begin{tabular}{|c|c|c|c|c|c|c|c|}
\hline & coaxial & $\begin{array}{l}\text { Measured } \\
\text { Torque }\end{array}$ & $\begin{array}{l}\text { Computed } \\
\text { Torque }\end{array}$ & $\begin{array}{l}\text { Relative } \\
\text { Error }\end{array}$ & $\begin{array}{c}\text { Measured } \\
\text { Mixing } \\
\text { Time } \\
\end{array}$ & $\begin{array}{l}\text { Computed } \\
\text { Mixing } \\
\text { Time } \\
\end{array}$ & $\begin{array}{l}\text { Relative } \\
\text { Error }\end{array}$ \\
\hline No & & (N.m) & (N.m) & $(\%)$ & $(\min )$ & (min) & $(\%)$ \\
\hline \multirow{2}{*}{1} & Scaba & 0.407 & 0.428 & 4.9 & \multirow{2}{*}{15.10} & \multirow{2}{*}{15.80} & \multirow{2}{*}{4.4} \\
\hline & Anchor & 0.953 & 0.986 & 3.3 & & & \\
\hline \multirow[b]{2}{*}{2} & Scaba & 0.568 & 0.593 & 4.2 & \multirow[b]{2}{*}{14.10} & \multirow[b]{2}{*}{14.60} & \multirow[b]{2}{*}{3.4} \\
\hline & Anchor & 0.908 & 0.945 & 4.0 & & & \\
\hline \multirow[b]{2}{*}{3} & Scaba & 0.677 & 0.712 & 4.9 & \multirow[b]{2}{*}{12.20} & \multirow[b]{2}{*}{12.80} & \multirow[b]{2}{*}{4.7} \\
\hline & Anchor & 0.828 & 0.868 & 4.6 & & & \\
\hline \multirow[b]{2}{*}{4} & Scaba & 0.774 & 0.810 & 4.4 & \multirow[b]{2}{*}{11.60} & \multirow[b]{2}{*}{12.16} & \multirow[b]{2}{*}{4.6} \\
\hline & Anchor & 0.788 & 0.831 & 5.2 & & & \\
\hline \multirow[b]{2}{*}{5} & Scaba & 0.912 & 0.956 & 4.8 & \multirow[b]{2}{*}{9.10} & \multirow[b]{2}{*}{9.50} & \multirow[b]{2}{*}{4.2} \\
\hline & Anchor & 0.853 & 0.895 & 4.7 & & & \\
\hline
\end{tabular}

\subsubsection{Results and Discussion}

\subsubsection{Design of Experiment}

The Box-Bhenken model was used to determine the most efficient coaxial mixer among the investigated coaxial agitators with respect to the mixing time and specific power drawn. By fitting the experimental data for mixing time and specific power drawn to the second-order polynomial regression, two quadratic equations as the response functions for both mixing time $\left(Y_{1}\right)$ and specific power drawn $\left(Y_{2}\right)$ were obtained:

$$
Y_{1}=11.90-1.82 X_{1}+3.70 X_{2}-2.03 X_{3}+1.59 X_{1} X_{2}+5.06 X_{1}^{2}-1.45 X_{2}^{2}-3.79 X_{3}^{2}
$$


$Y_{2}=659.87+148.16 X_{1}+48.46 X_{2}+362.36 X_{3}+111.06 X_{1} X_{3}-142.76 X_{2} X_{3}-214.95 X_{1}^{2}+$ $139.31 X_{3}^{2}$

The results of the ANOVA tabulated in Tables (5.3-4) and (5.3-5) were used to test the statistical significance of the generated quadratic equations to predict the mixing time and specific power consumption.

Table (5.3-4). ANOVA test for response function (mixing time) $Y_{1}=11.90-1.82 X_{1}+$ $3.70 X_{2}-2.03 X_{3}+1.59 X_{1} X_{2}+5.06 X_{1}^{2}-1.45 X_{2}^{2}-3.79 X_{3}^{2}\left(R^{2}=0.9840\right)$.

\begin{tabular}{cccccc}
\hline Source & Sum of & Degree of & $\begin{array}{c}\text { Mean } \\
\text { Square }\end{array}$ & F-ratio & P-value \\
& Squares & freedom & & & Prob $>$ F \\
\hline Model & 613.80 & 14 & 43.84 & 28.78 & $<0.0001$ \\
$X_{1}$ & 39.53 & 1 & 39.53 & 25.95 & 0.0002 \\
$X_{2}$ & 164.21 & 1 & 164.21 & 107.80 & $<0.0001$ \\
$X_{3}$ & 49.25 & 1 & 49.25 & 32.33 & $<0.0001$ \\
$X_{1} X_{3}$ & 10.14 & 1 & 10.14 & 6.66 & 0.0218 \\
$X_{1}{ }^{2}$ & 165.86 & 1 & 165.86 & 108.88 & 0.0001 \\
$X_{2}{ }^{2}$ & 13.72 & 1 & 13.72 & 9.01 & 0.0095 \\
$X_{3}{ }^{2}$ & 93.28 & 1 & 93.28 & 61.23 & $<0.0001$ \\
\hline
\end{tabular}

Table (5.3-5). ANOVA test for response function (power consumption) $Y_{2}=659.87+$ $148.16 X_{1}+48.46 X_{2}+362.36 X_{3}+111.06 X_{1} X_{3}-142.76 X_{2} X_{3}-214.95 X_{1}^{2}+139.31 X_{3}^{2}\left(R^{2}\right.$ $=0.9719)$.

\begin{tabular}{cccccc}
\hline Source & $\begin{array}{c}\text { Sum of } \\
\text { Squares }\end{array}$ & $\begin{array}{c}\text { Degree of } \\
\text { freedom }\end{array}$ & Mean Square & F-ratio & $\begin{array}{c}\text { P-value } \\
\text { Prob }>\text { F }\end{array}$ \\
\hline Model & $2.55 \times 10^{6}$ & 14 & $1.81 \times 10^{5}$ & 34.55 & $<0.0001$ \\
$X_{1}$ & $2.63 \times 10^{5}$ & 1 & $2.63 \times 10^{5}$ & 50.21 & $<0.0001$ \\
$X_{2}$ & 288.82 & 1 & 2818.82 & 5.37 & 0.0361 \\
$X_{3}$ & $1.57 \times 10^{6}$ & 1 & $1.57 \times 10^{6}$ & 300.34 & $<0.0001$ \\
$\mathrm{X}_{1} \mathrm{X}_{3}$ & 49341.74 & 1 & 4934.740 & 9.41 & 0.0084 \\
$X_{2} X_{3}$ & 22.52 & 1 & 22.52 & 3.07 & 0.0015 \\
$X_{1}{ }^{2}$ & $2.99 \times 10^{5}$ & 1 & $2.99 \times 10^{5}$ & 57.13 & $<0.0001$ \\
$X_{3}{ }^{2}$ & $1.25 \times 10^{5}$ & 1 & $1.25910^{5}$ & 24.00 & 0.0002 \\
\hline
\end{tabular}


The Fisher's F-test (Khayet et al., 2011) and values of probability less than 0.05 in the aforementioned tables were utilized to determine the significance of the models and each coefficient in Equations (5.3-2) and (5.3-3). It can be seen that the models were highly significant with a probably value of $P<0.05$. The coefficient of determination $\left(R^{2}\right)$ was used to evaluate the goodness of the fit. The high values of this coefficient $\left(R^{2}=0.9840\right.$ for mixing time and $R^{2}=0.9719$ for specific power dawn) show that the mixing time and specific power drawn can be predicted very well by Equations (5.3-2) and (5.3-3), respectively. Figure (5.3-2) depicts the collected experimental data verses the predicted ones obtained from Equations (5.3-2) and (5.3-3) for the mixing time and power drawn. These results showed a quite satisfactory agreement between the observed and predicted data for both mixing time and specific power drawn of the coaxial mixers.

(a)

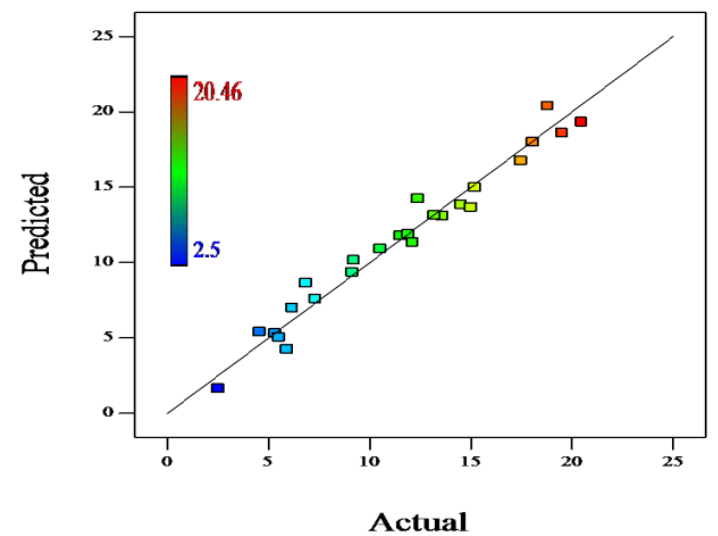

(b)

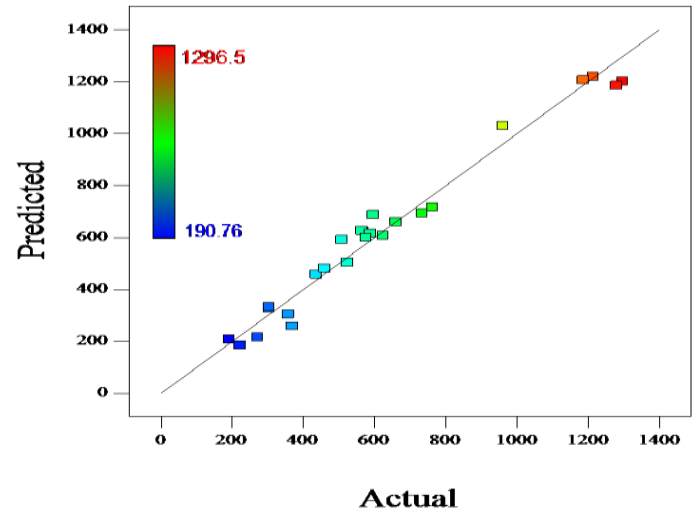

Figure (5.3-2). Predicted values versus the experimental data for (a) mixing time $(s)$ and (b) specific power consumption $\left(\mathrm{W} / \mathrm{m}^{3}\right)$.

To keep the second-order equations (response functions) into perspective, the threedimensional $(3 D)$ response surfaces and two-dimensional (2D) contour plots, which 
demonstrate the response function of two interacting factors at a time while the other variables are fixed, were plotted and presented in Figure (5.3-3). Figure (5.3-3)a demonstrates the interaction between the independent variables of coaxial configuration $\left(X_{1}\right)$ and xanthan gum concentration $\left(X_{2}\right)$, and their effects on the mixing time. As can be noted in this figure, there was a significant interaction between these two independent variables affecting the mixing time. The ANOVA analysis considered this interaction by including $X_{1} X_{2}$ term in Equation (5.3-2) due to a small $P$-value $(P<0.05)$ for $X_{1} X_{2}$, as reported in Table (5.3-4). Figure (5.3-3)a also shows that by changing the central impeller from an axial-flow pitched blade turbine to a radial-flow Scaba or Rushton turbine, the mixing time was decreased. This shows an agreement with that reported by Foucault et al. (2006) and Pakzad et al. (2013c). It must also be mentioned that the DSAC (double Scaba impellers-anchor coaxial) mixer showed the lowest mixing time compared to the DPAC (double pitched blade turbines-anchor coaxial) mixer and the DRAC (double Rushton turbines-anchor coaxial) mixer within the experimental range studied in this paper.

The interaction between the independent variables of the coaxial configuration $\left(X_{I}\right)$ and the central impeller speed $\left(X_{3}\right)$ was significant due to a small $P$-value $(P<0.05)$, and thus $X_{1} X_{3}$ were included in the response function of the specific power drawn as it can be seen in Equation (5.3-3). The three-dimensional ( $3 D)$ and two-dimensional (2D) contour plots of the effect of $X_{1} X_{3}$ are shown in Figure (5.3-3) b. These results show that the specific power drawn for a double central impeller-anchor coaxial mixer was strongly dependent on the central impeller speed $\left(X_{3}\right)$. Indeed, to reach a higher rotational speeds of the dual central impellers more power was required. On the other hand, with increasing 
the speed of the central impeller in co-rotating mode for the three coaxial mixers studied in our experiments, the power consumption of the double pitched blades-anchor coaxial (DPAC) mixer was less than that for the other two coaxial mixers. Such a behavior demonstrated a good agreement with this fact that the axial-flow impellers are known to be the most energy-efficient impellers in term of power consumption (Pakzad et al., 2013c; Cullen, 2009). Furthermore, the interaction between the xanthan gum concentration $\left(X_{2}\right)$ and the central impeller speed $\left(X_{3}\right)$ was significant due to a small $P$ value $(P<0.05)$. Therefore, the interaction between these two independent variables $\left(X_{2} X_{3}\right)$ were incorporated into Equation (5.3-3). The influence of $X_{2}$ and $X_{3}$ on the specific power drawn is shown in Figure (5.3-3) c. This Figure illustrates that the specific power consumption of the coaxial mixer was increased by increasing the xanthan gum concentration while the central impeller speed $\left(X_{3}\right)$ was kept constant. This concentration-dependent behavior could be explained by this fact that more energy is consumed by the impeller rotated in a fluid with a higher apparent viscosity.

(a)
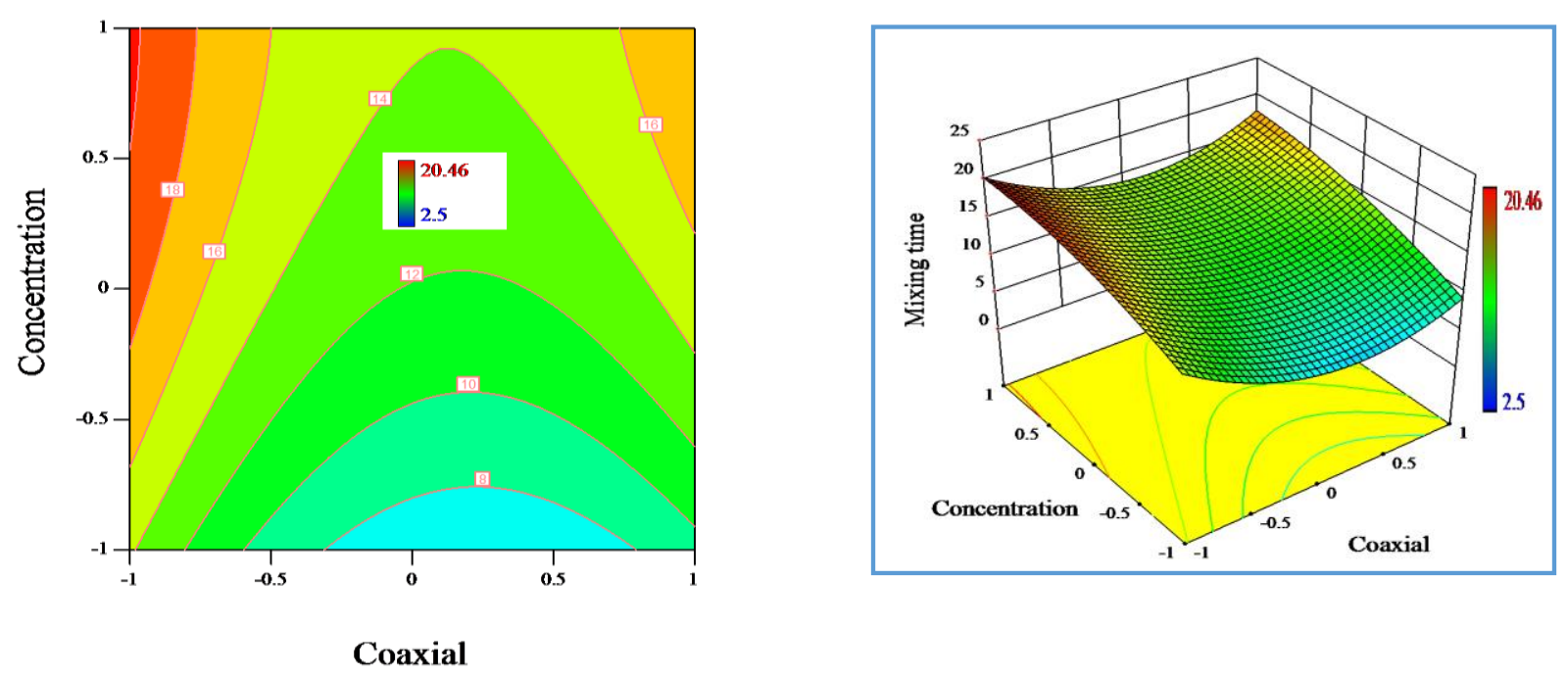
(b)
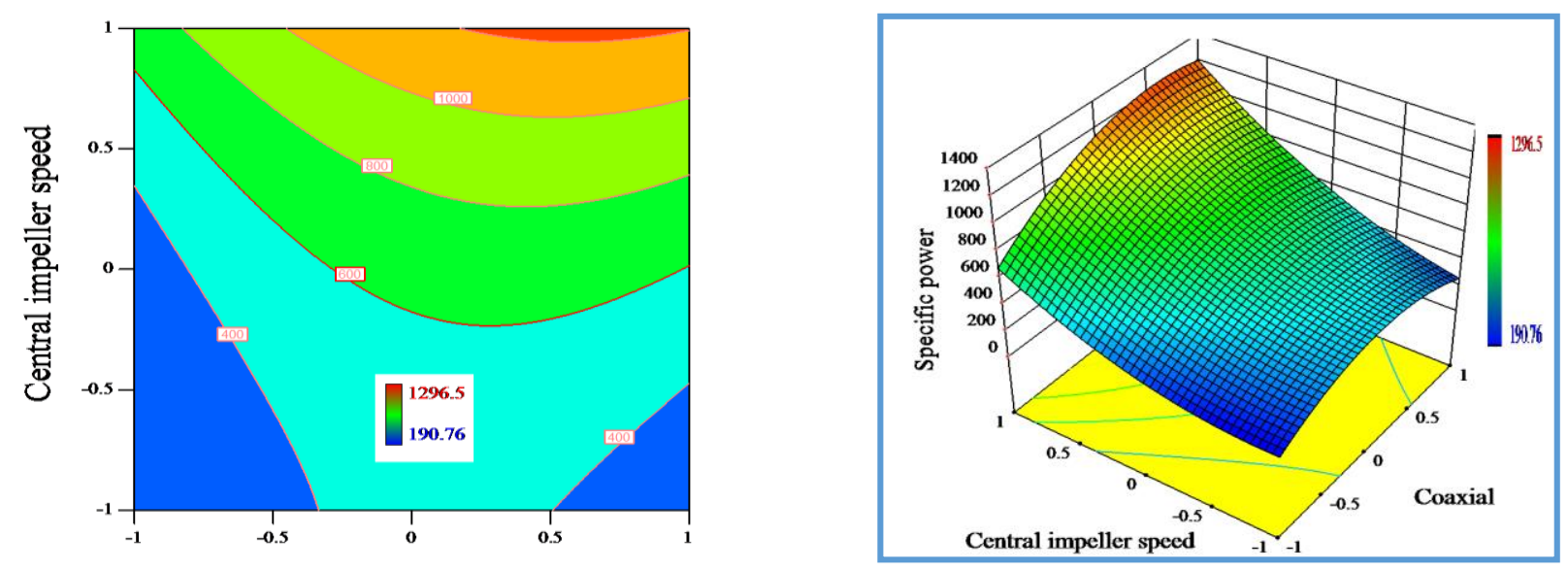

Coaxial

(c)
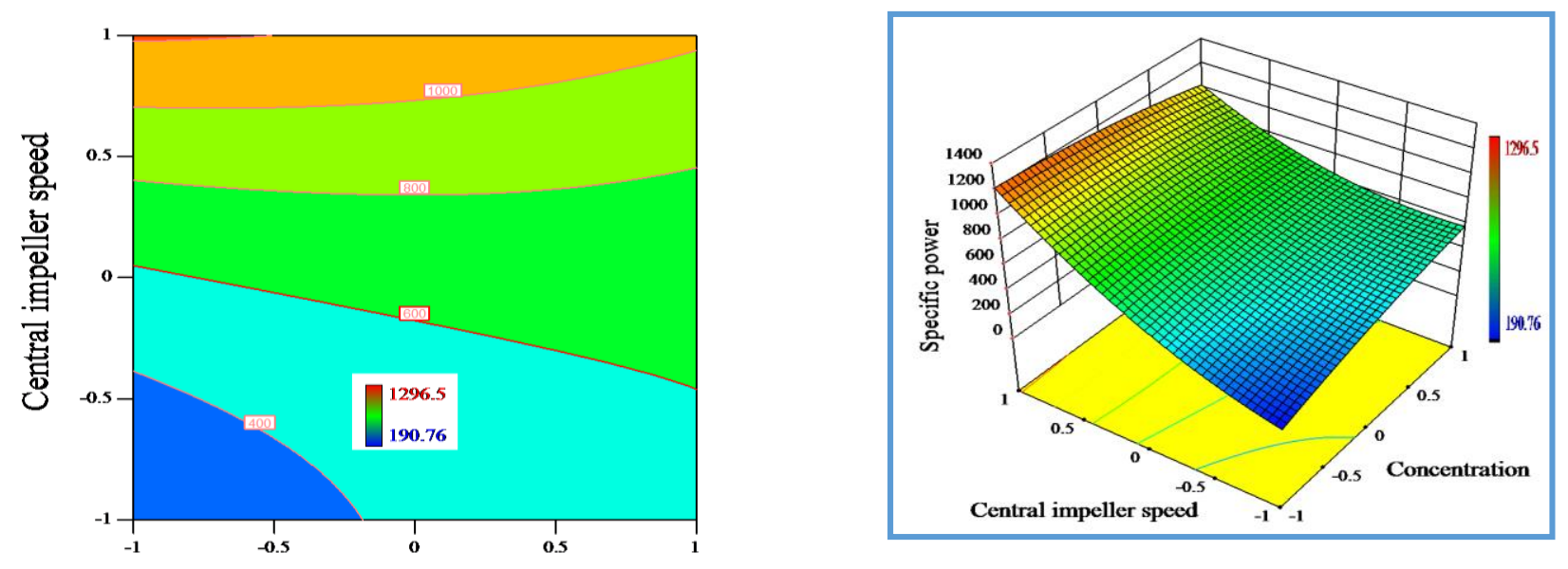

\section{Concentration}

Figure (5.3-3). Response surface methodology (RSM) results showing mixing time and specific power drawn as a function of two independent variables (other variables were fixed): (a) coaxial mixer $\left(X_{1}\right)$ and concentration $\left(X_{2}\right)$, (b) coaxial mixer $\left(X_{1}\right)$ and central impeller speed $\left(X_{3}\right),(\mathbf{c})$ concentration $\left(X_{2}\right)$ central impeller speed $\left(X_{3}\right)$. 


\subsection{2 Flow Pattern}

In this section, the flow domains generated by the DSAC, DRAC, and DPAC coaxial mixers are analyzed. Figure (5.3-4) and Figure (5.3-5) depict the velocity vectors and streamlines for these three coaxial mixers at a speed ratio of $R_{n}=8\left(N_{c}=240 \mathrm{rpm}, N_{a}=30\right.$ rpm) in the co-rotating mode. These CFD results demonstrate the major flow features such as the circulation loops, discharge streams, contributions of the radial and axial velocities in the flow domain, and the flow loops formed above and below the central impeller inside the agitated vessel. The flow pattern created by the DSAC and DRAC mixers were similar since the central impellers used in these two configurations were radial-flow impellers. However, as expected, the flow pattern of the DPAC mixer with the axial-flow central impellers (dual pitched blade turbine) was different than those of the DSAC and DRAC. It can be seen from Figures (5.3-4)a, (5.3-4)b, (5.3-5)a, and (5.35)b that both DSAC and DRAC mixers generated similar stable parallel flow patterns (Rutherford et al., 1996) at the impeller spacing equal to $T / 2$. In fact, in the parallel flow pattern, a flow jet was directed by the individual upper and lower impellers toward the tank wall at a small angle to the horizontal plane, and the flow was then divided into the upward and downward flow along the vessel wall. The split flows then returned to the central impeller through the circulation loops at the upper and the lower part of the vessel. In fact, the DSAC and DRAC coaxial mixers generated two loops above the upper impellers (Scaba or Rushton), four loops between the two central high speed impellers, and two loops below the lower impeller. This phenomenon promoted the continuous and steady motion of the fluid without any compartmentalization from the lower part to the upper part of the tank resulting in a more efficient mixing system. 
For the DPAC mixer as shown in Figures (5.3-4) c and (5.3-5) c, one large circulation loop was generated by both pitched blade impellers within the mixing tank and two small circulation loops created by each central impeller. This type of the flow pattern has also been reported for the mixing of the Newtonian fluids with dual pitched blade turbines in turbulent flow by Baudou et al., (1997) and Kresta et al. (1993a) for the impeller spacing greater than $T / 2$. They observed that the flow stream from the upper impeller was split in two parts: one part was drawn by the lower impeller and second part was directed radially to the wall before rising close to the tank wall towards the upper part of the vessel. In their system, dead zones were noticed in the upper part and in the bottom of the vessel indicating insufficient circulations in the agitated system. As a consequence, the pitched blade turbines mounted on the same shaft created a larger circulation loop. The generation of a larger axial circulation modified the flow patterns induced by each impeller, i.e. made them more radial than axial. The same phenomenon was noticed for the mixing of the yield-pseudoplastic fluid by the double pitched blade turbines in combination with an anchor in this study without extensive dead zones at the bottom of tank as shown in Figure (5.3-4) c and (5.3-5) c. The development of the dead zone at the bottom of the vessel were decreased for three coaxial mixer configurations due to the rotation of the anchor impeller. This kind of flow pattern with two secondary loops under each impeller is considered as an unstable flow leading to a less efficient mixing operation. 
(a)
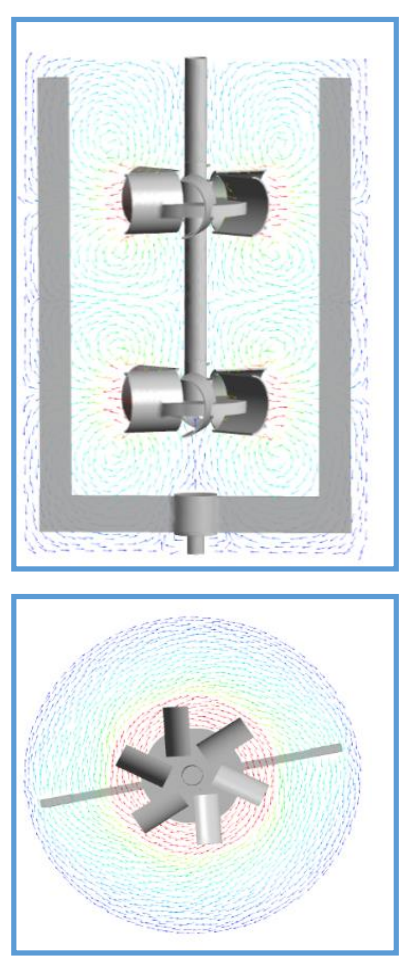

(b)
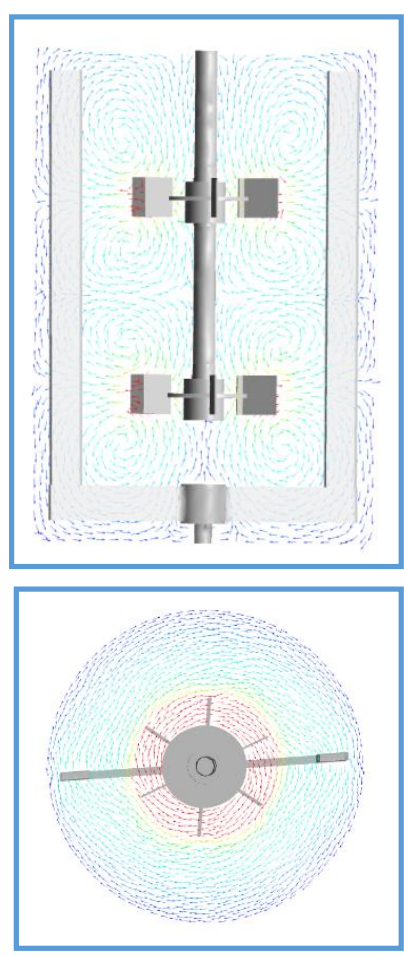

(c)
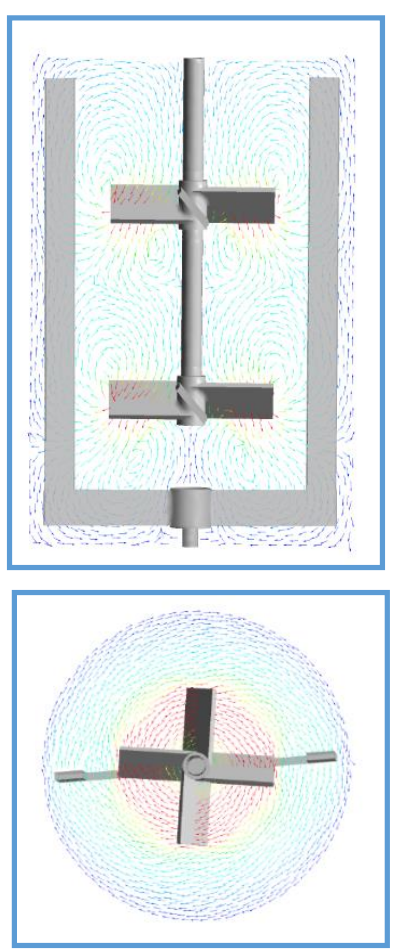

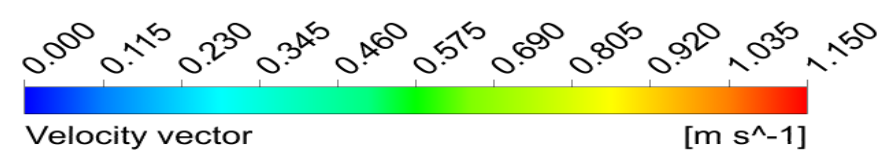

Figure (5.3-4). Vertical and horizontal velocity $(\mathrm{m} / \mathrm{s})$ vector plots at $R_{n}=8\left(N_{c}=240\right.$ rpm and $N_{a}=30 \mathrm{rpm}$ ), and 1.5\% xanthan gum solution for: (a) double Scaba-anchor coaxial mixer (DSAC) mixer, (b) double Rushton-anchor coaxial (DRAC) mixer, and (c) double pitched blade-anchor coaxial (DPAC) mixer. 
(a)

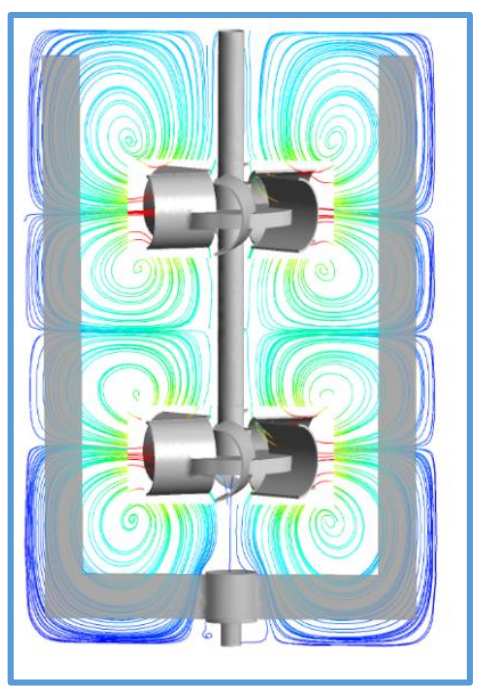

(b)

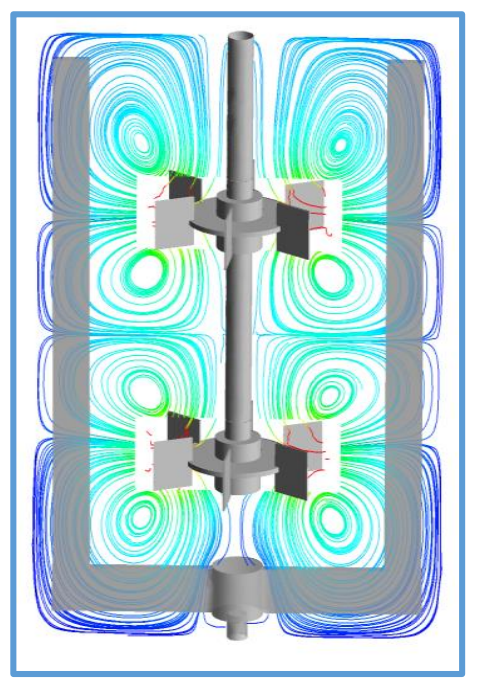

(c)

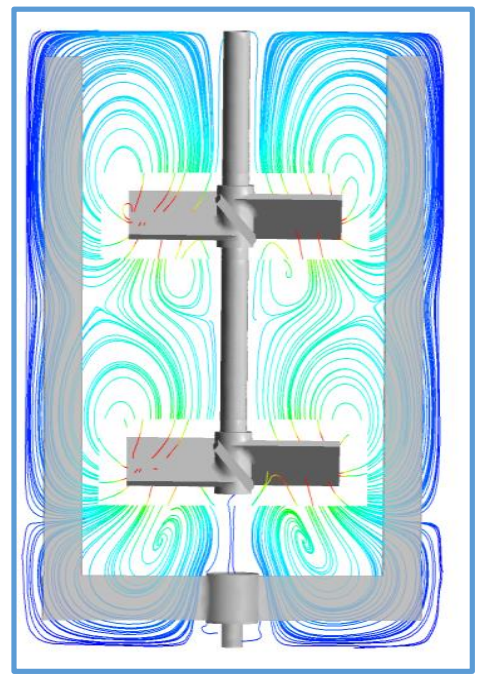

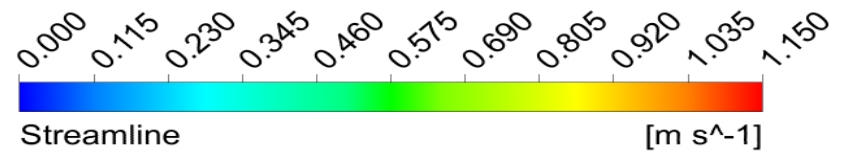

Figure (5.3-5). Streamline plots at $R_{n}=8\left(N_{c}=240 \mathrm{rpm}\right.$ and $\left.N_{a}=30 \mathrm{rpm}\right)$, and $1.5 \%$ xanthan gum solution for: (a) double Scaba-anchor coaxial mixer (DSAC) mixer, (b) double Rushton-anchor coaxial (DRAC) mixer, and (c) Double pitched blade-anchor coaxial (DPAC) mixer.

To quantitatively analyze the hydrodynamics of the DSAC, DRAC, and DPAC mixers , the average radial, axial, and tangential velocity profiles along the tank wall (normalized by the tank height) at a radial position of $2 r / R=0.45$ and $R_{\mathrm{n}}=8\left(N_{c}=240 \mathrm{rpm}, N_{a}=30\right.$ rpm) for these three coaxial mixers are depicted in Figure (5.3-6). Figure (5.3-6) a shows that the axial circulations were generated by three coaxial mixers in the regions located above and below the central impellers, and they were more pronounced compared to those for the single central impeller-anchor coaxial mixer (Kazemzadeh et al., 2016b). However, the magnitude of the axial velocity for the DPAC mixer was higher $\left(V_{a}=0.17\right.$ 
$\left.V_{\text {tip }}\right)$ than those for the other two as expected. As shown in Figure (5.3-6) b, the highest radial velocity $\left(V_{r}=0.13 V_{\text {tip }}\right)$ was achieved by the DSAC mixer. Figure (5.3-6) b also demonstrates the generation of two circulation loops for the DPAC mixer, one above and one below the pitched blade turbine impeller, with an axial circulation barrier between two loops. This flow pattern was similar to that reported by Farhat et al. (2007) for the Rushton turbine. This confirms the radial discharge flow for the pitched blade turbine in the mixing of yield-pseudoplastic fluids in the laminar regime. Rudolph et al. (2009) also reported that the pitched blade turbine acted as a radial impeller at a low Reynolds number. It must be mentioned that this circulation barrier promoted the axial compartmentalization, which was more pronounced for the DPAC mixer than those for the other two coaxial mixers, which will be discussed later. Furthermore, Figure (5.3-6) c depicts the tangential velocity profiles for three coaxial mixers investigated in this study. This figure clearly illustrates that the highest tangential velocity $\left(V_{t}=0.95 V_{t i p}\right)$ was attained by the DPAC mixer. However, a consistent tangential velocity distribution particularly close to the tips of the impellers with a pick value of $V_{t}=0.65 V_{\text {tip }}$ was generated by DSAC mixer system. 
(a)

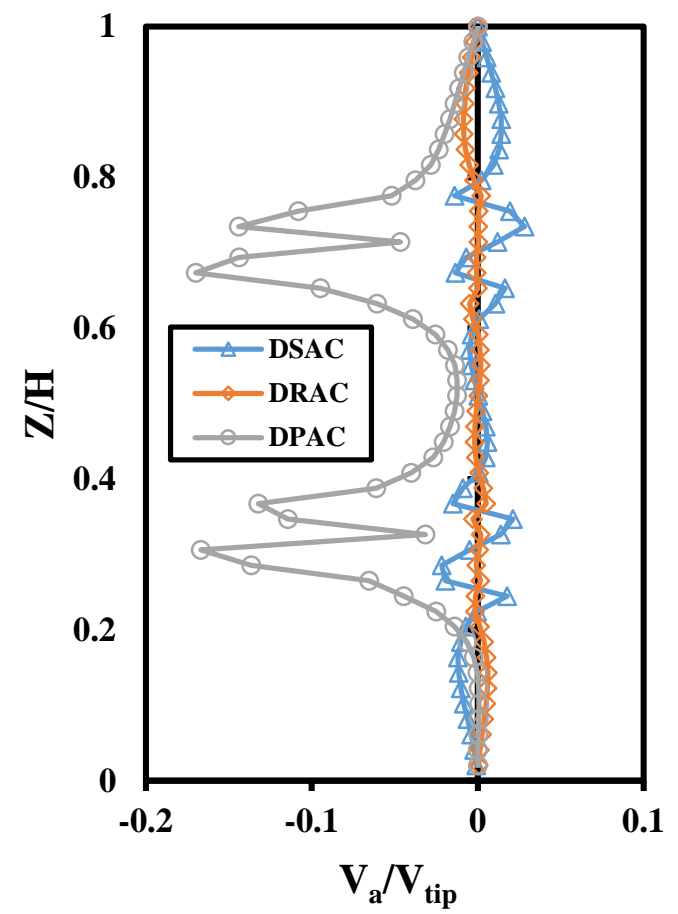

(b)

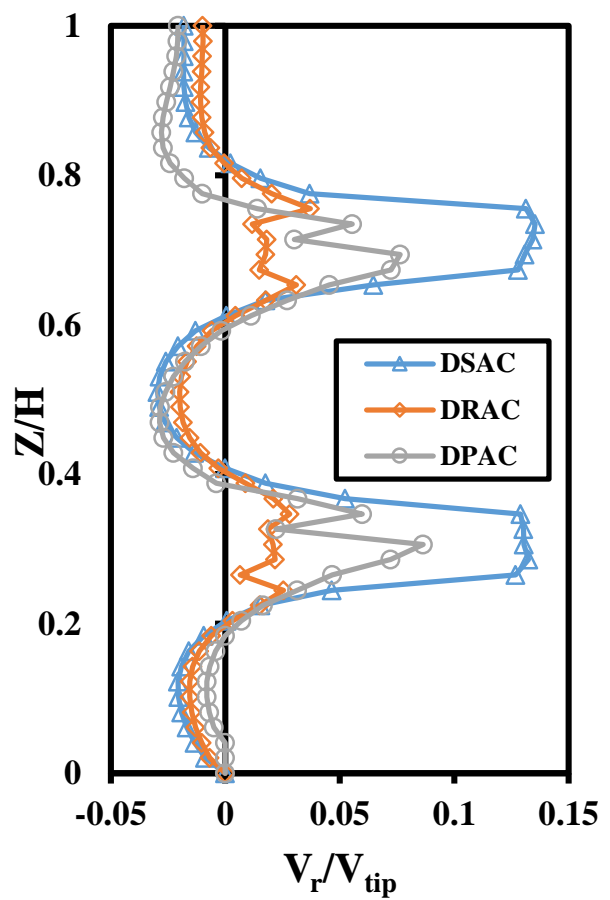

(c)

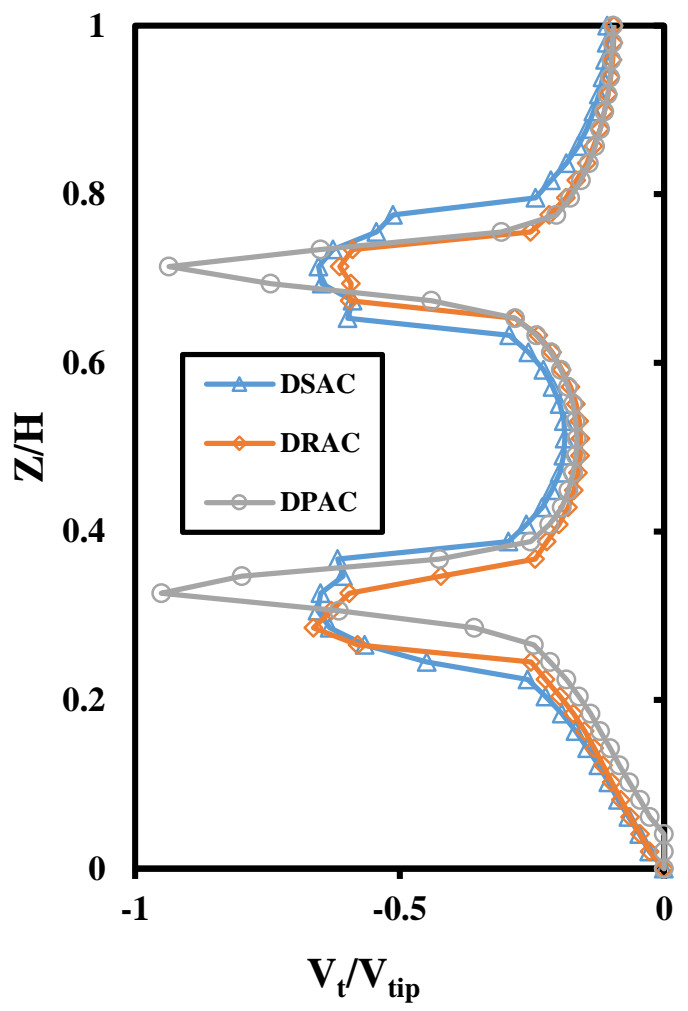

Figure (5.3-6). Normalized velocity profiles along the tank wall at $2 r / T=0.45, R_{n}=8$ $\left(N_{c}=240 \mathrm{rpm}\right.$ and $\left.N_{a}=30 \mathrm{rpm}\right)$, and $1.5 \%$ xanthan gum solution: (a) axial velocity (b) radial velocity, and (c) tangential velocity. 
To evaluate the performances of the coaxial agitated mixers in depth, the radial profiles of the axial, radial, and tangential velocities above the lower impeller $(Z / H=0.36)$ and underneath the lower impeller $(Z / H=0.24)$ are presented in Figure $(5.3-7)$. The radial positions from the shaft to the tip of the anchor were normalized by the tank diameter ( $T$ $=0.5 \mathrm{~m})$, and velocities were normalized by the central impeller tip speed $\left(V_{t i p}=2.2608\right.$ $\mathrm{m} / \mathrm{s}$ ). Figures (5.3-7) a and (5.3-7) b illustrate that the magnitude of the axial velocity for the DPAC mixer was greater than those for the other two mixing systems for both radial profiles. It can be noted that the DPAC generated the maximum axial velocity $\left(V_{a}=0.12\right.$ $\left.V_{t i p}\right)$ at the radial location of $2 r / T=0.45$ in the downward direction for both radial profiles while the minimum of the axial velocity profiles was shifted towards the tank wall. The radial velocity profiles computed at $\mathrm{Z} / H=0.36$ and $\mathrm{Z} / \mathrm{H}=0.24$ above and below of the lower impeller, for three configurations of the coaxial mixers are presented in Figures (5.3-7) c and (5.3-7) d. It can be observed that the highest radial velocity was achieved by the DPAC mixer. This could be explained due to closeness of the defined location to the tip of the pitched blade turbines. It is interesting that the radial velocity direction above the lower impeller changed for the DPAC mixer. This was due to the fact that the downward discharged flow of the upper impeller was split into two streams. One stream was drawn by the lower impeller; created a smaller circulation loop in comparison to the other one, which was under the upper impeller close to the shaft; and the second stream was pumped towards the vessel walls to create the upper loop [see Figures (5.3-4) $\mathrm{c}$ and (5.3-5) c]. Furthermore, Figures (5.3-7) e and (5.3-7)f show the tangential velocity profiles for three mixers at two locations, above and below the lower impeller. These figures demonstrate that DSAC coaxial mixer achieved the maximum magnitude of 
tangential velocity with the pick values of $V_{t} / V_{\text {tip }}=0.47$ and $V_{t} / V_{t i p}=0.72$ at $2 r / T=.45$.

Also, it can be noted that the tangential velocities became weaker as they were shifted from the shaft to the tip of the anchor impeller close to the vessel

(a)

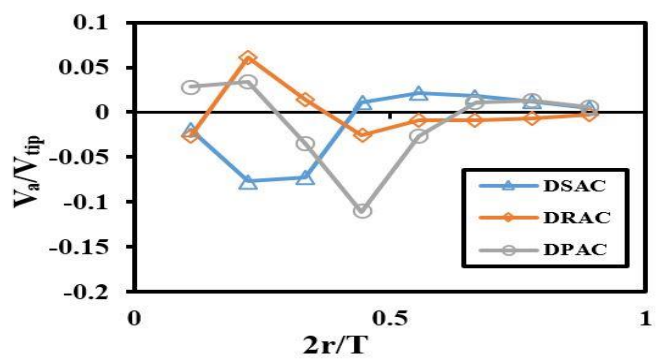

(c)

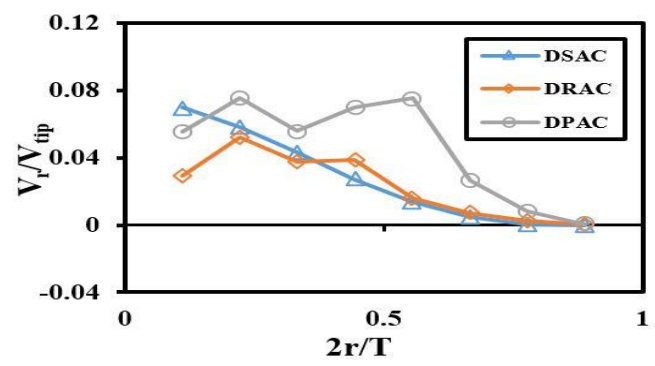

(e)

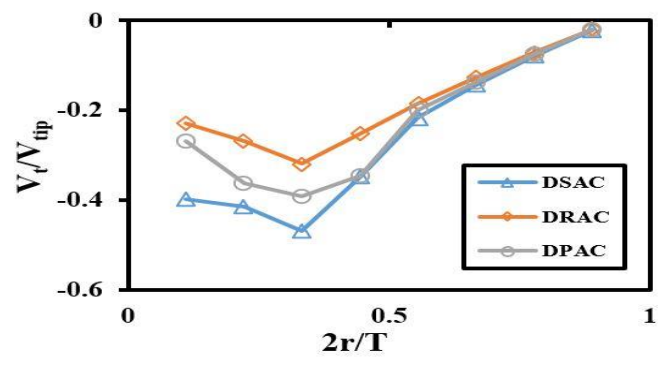

(b)

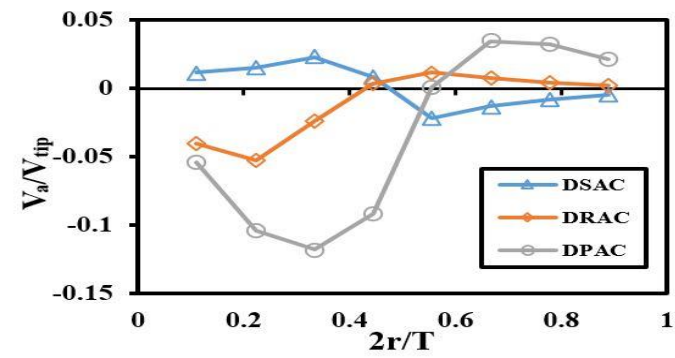

(d)

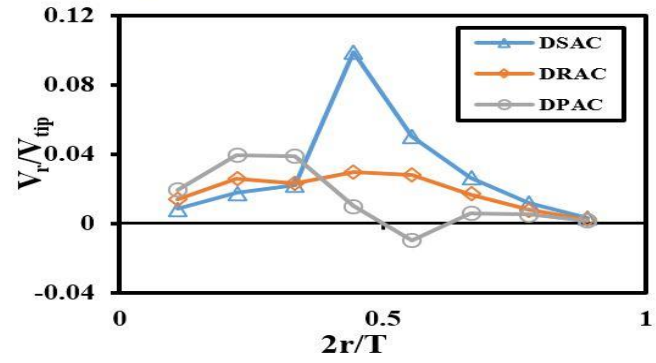

(f)

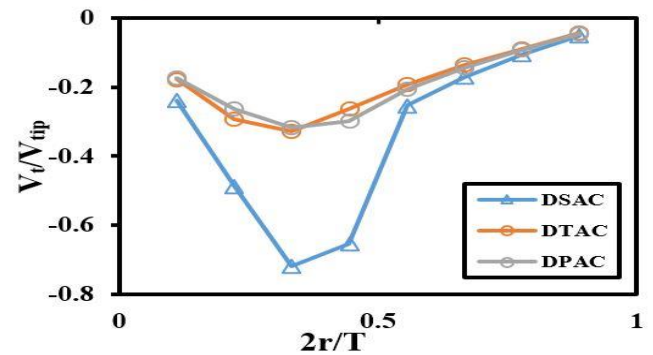

Figure (5.3-7). Radial profiles of axial, radial, and tangential velocities at $R_{n}=8\left(N_{c}=\right.$ $240 \mathrm{rpm}$ and $N_{a}=30 \mathrm{rpm}$ ), and $1.5 \%$ xanthan gum solution : (a) axial velocity under the lower impeller along the line parallel to the $x$-axis with $Z / H=0.24$, (b) axial velocity above the lower impeller along the line parallel to the $x$-axis with $Z / H=0.36$, (c) radial velocity under the lower impeller along the line parallel to the $x$-axis with $Z / H=0.24$, , (d) radial velocity above the lower impeller along the line parallel to the $x$-axis with $Z / H$ $=0.36$, (e) tangential velocity under the lower impeller along the line parallel to the $x$ axis with $Z / H=0.24$, and (f) tangential velocity above the lower impeller along the line parallel to the $x$-axis with $Z / H=0.36$. 
Figure (5.3-8) depicts the velocity contour plots at $R_{n}=8\left(N_{c}=240 \mathrm{rpm}, N_{a}=30 \mathrm{rpm}\right)$ in the co-rotating mode for three coaxial agitated mixers studied in this paper. As can be noticed in Figure (5.3-8) a, the maximum magnitude of the axial velocity inside the tank was attained by the DPAC mixer. However, due to the interactions between the circulation loops (unstable flow), the axial circulation profile for the DPAC mixer was less uniform than those for the other two coaxial mixers. This figure also shows that a more uniform axial circulation was achieved by the DSAC mixer throughout the vessel due to the stable parallel flow of four rings for each impeller, two above and two below the impeller.

The radial velocity contours of the three investigated coaxial mixers are illustrated in Figure (5.3-8) b. As it was expected, for the DPAC mixer, the corresponding radial velocities were different from those generated by the other two impellers. Due to an unstable flow, the highest radial velocities induced by the DPAC mixer were restricted to the regions close to each central impeller. The radial velocity patterns created by the DSAC and DRAC mixers were similar but they were different in magnitude, especially in the regions between the two central impellers.

Finally, the tangential velocity profiles attained by the three coaxial mixers are shown in Figure (5.3-8) c. This figure illustrates that the magnitude of the tangential velocity obtained by the DSAC was higher than those by the DRAC and DPAC mixers. As it was previously discussed, the magnitude of the tangential velocity increased from the shaft to the tip of the central impellers, and then decreased towards the vessel wall [Figures (5.37) e and (5.3-7) f]. It is worth mentioning that the generation of the tangential flow by the anchor in co-rotating mode due to the interaction between the two impellers of the 
coaxial mixing system can intensify the size of the well mixed region (cavern) resulting in higher pumping in the axial direction(Rivera et al., 2006). It can be seen from Figure (5.3-8) $\mathrm{c}$ that the interaction between the central impeller and the anchor was more pronounced (i.e. larger cavern size) for the DSAC mixer than the other two mixers.

(a)
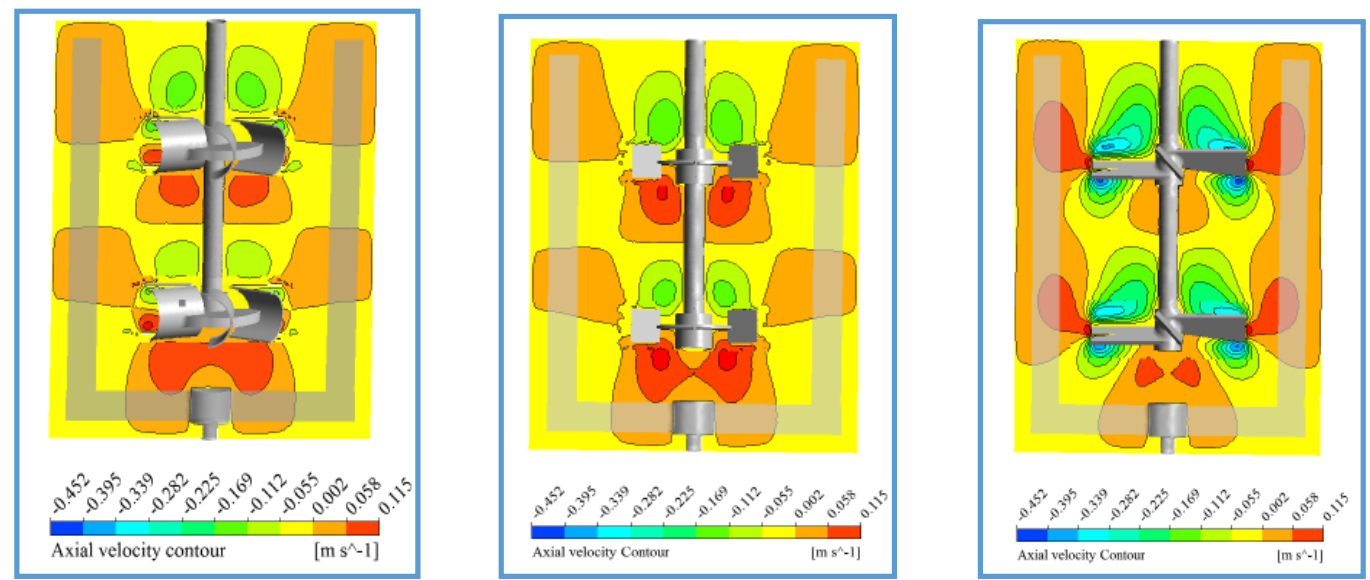

(b)
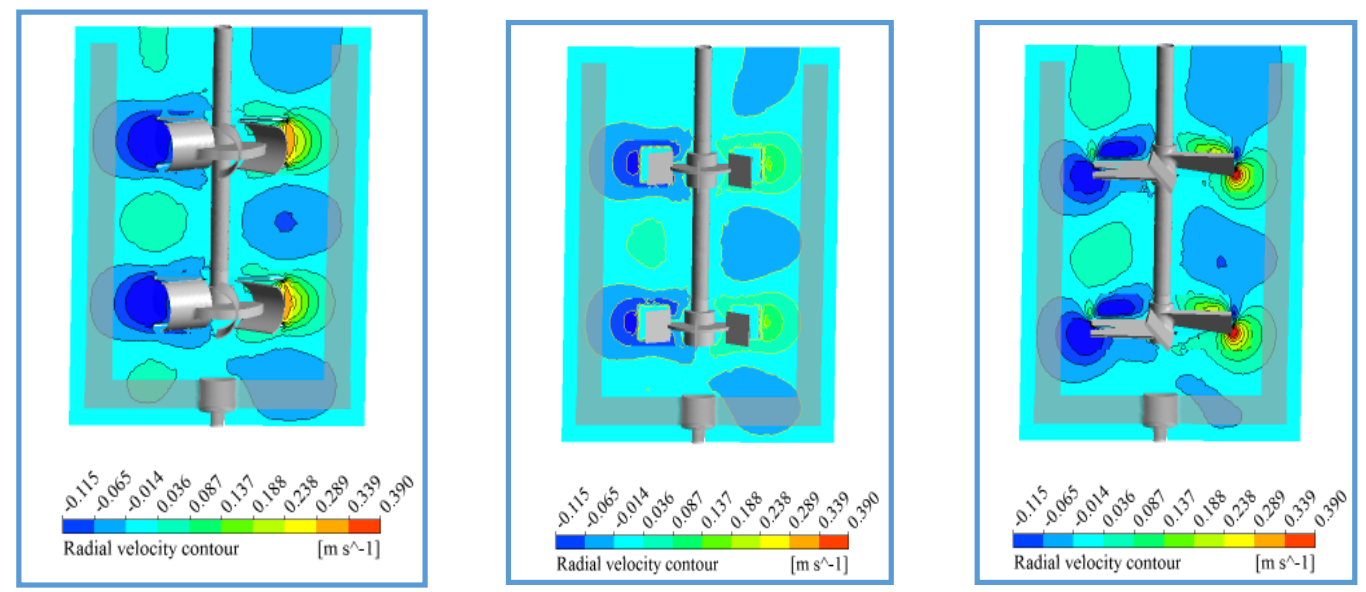
(c)
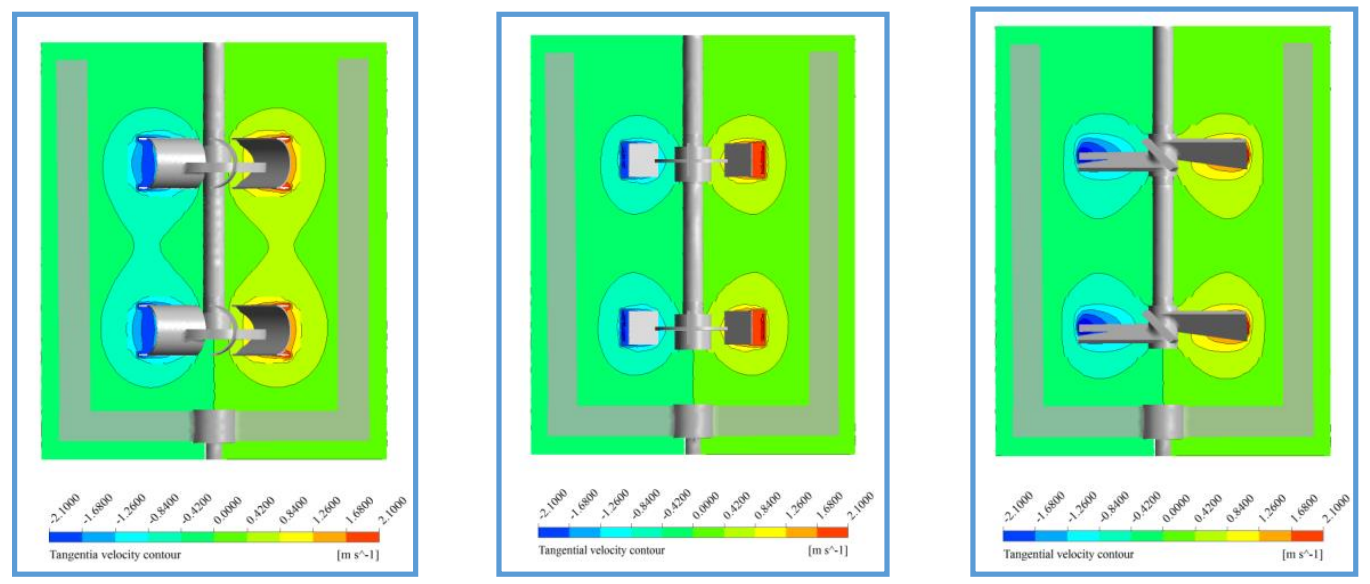

Figure (5.3-8). Velocity (m/s) contour plots at $R_{n}=8\left(N_{c}=240 \mathrm{rpm}\right.$ and $\left.N_{a}=30 \mathrm{rpm}\right)$, and 1.5\% xanthan gum solution for: (a) axial velocity, (b) radial velocity, and (c) tangential velocity.

Since the apparent viscosity of the xanthan gum solution depends on the shear rate, the shear rate curves (normalized with the rotational speed of the coaxial mixer) were plotted along the tank height at $R_{n}=8\left(N_{c}=240 \mathrm{rpm}, N_{a}=30 \mathrm{rpm}\right)$ in four radial positions $(2 \mathrm{r} / T$ $=0.25,2 r / T=0.45,2 r / T=0.55$, and $2 r / T=0.65)$ and are presented in Figures (5.3-9) a to (5.3-9) d, respectively. These graphs show that the shear rate increased in the radial direction from the shaft to the impeller tip and then decreased from the impeller tip towards the tank wall for all three configurations. The DRAC mixer induced the highest shear rate at $2 r / T$ with a maximum magnitude of $\dot{\gamma} / N_{C o}=60$ for the upper impeller and $\dot{\gamma} / N_{\text {co }}=52$ for the lower one. However, the shear rate profile generated by the DSAC mixer in the radial direction was more consistent than those by the other two mixers. Furthermore, these figures show that the shear rates generated by the DSAC and DPAC mixers between the anchor impeller and central impellers in co-rotating mode were higher compared to the other. However, DSAC produced a better and consistent 
distribution of the shear rate rather than DPAC one. Rudolph et al. (2009) reported the same trend for the mixing of non-Newtonian power law fluids with the DPAC mixer in the co-rotating and counter-rotating modes.

(a)

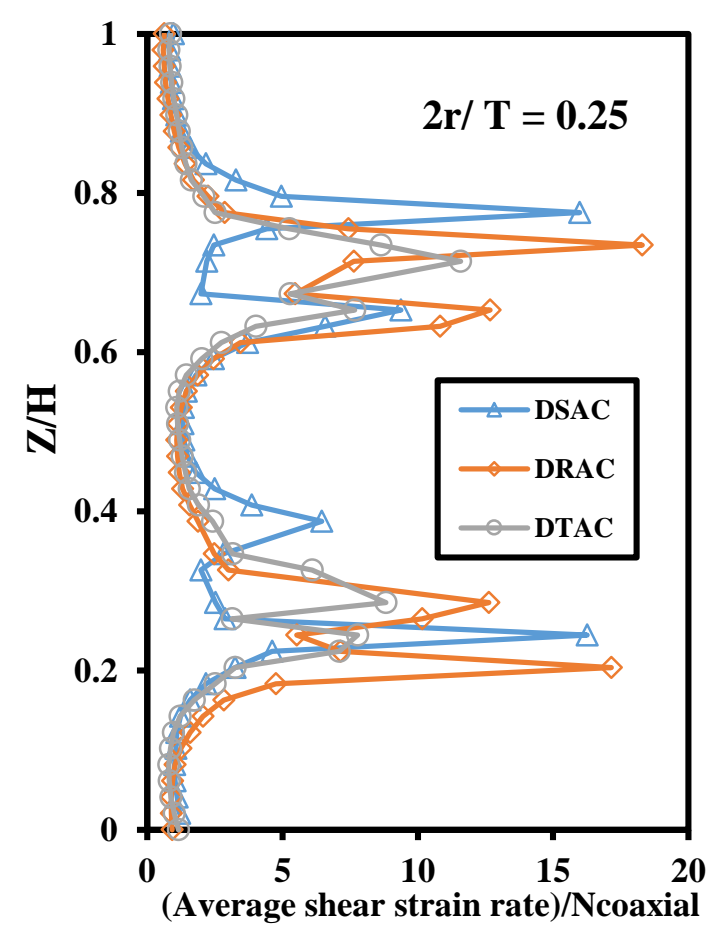

(b)

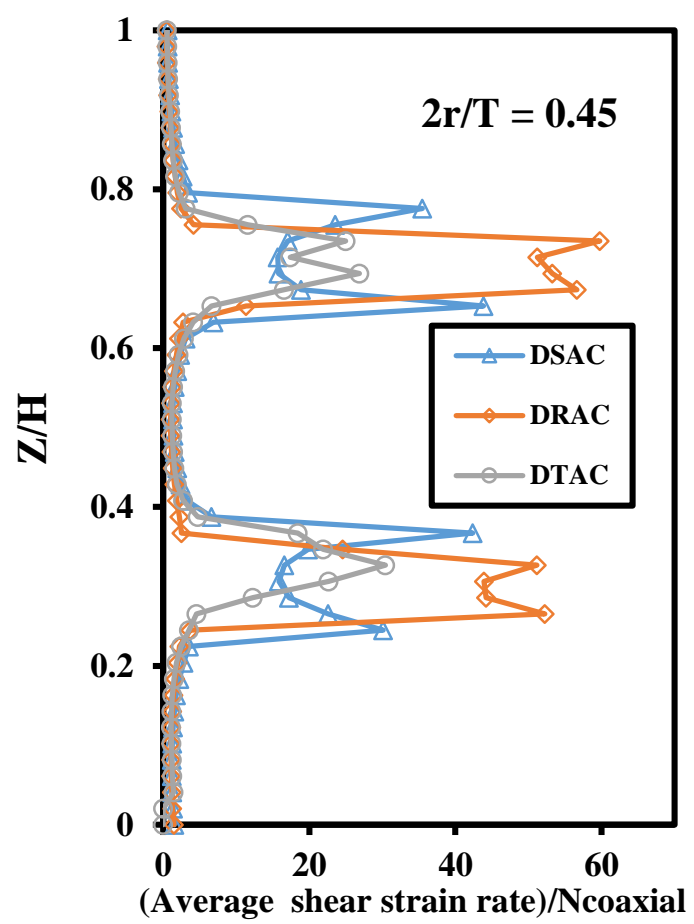


(c)

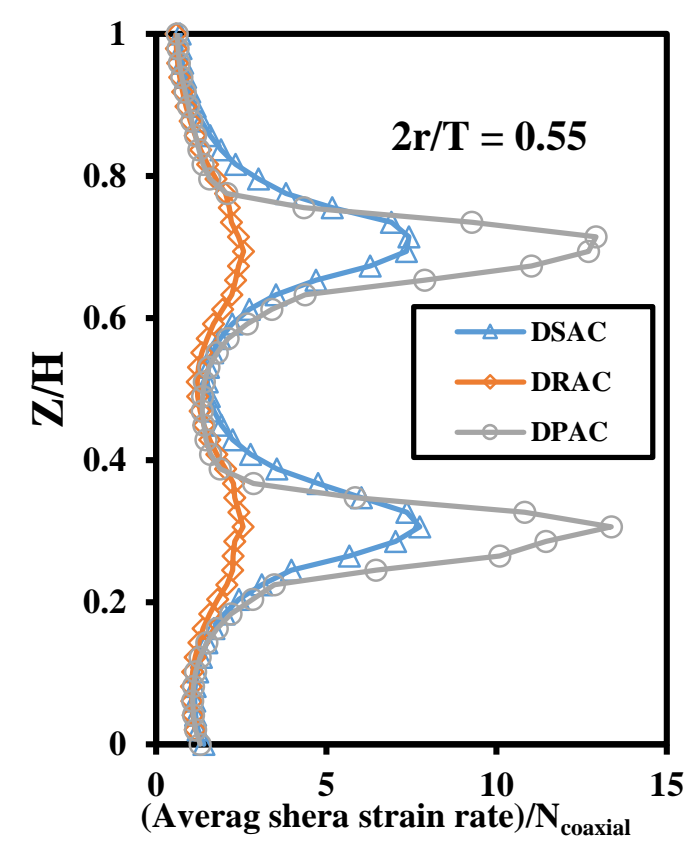

(d)

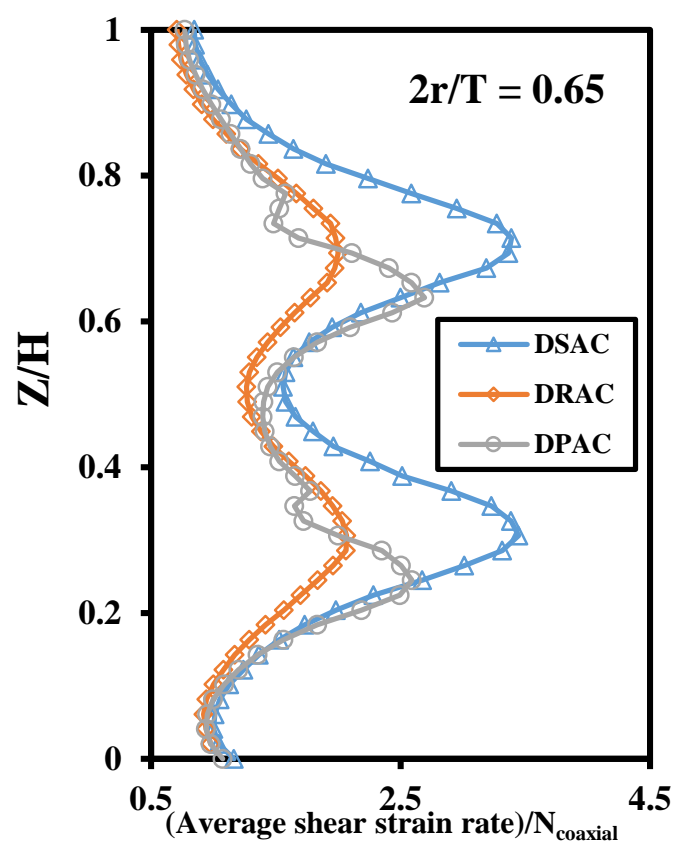

Figure (5.3-9). Dimensionless average shear rate plots at $R_{n}=8\left(N_{c}=240 \mathrm{rpm}\right.$ and $N_{a}=$ $30 \mathrm{rpm}$ ) and $1.5 \%$ xanthan gum solution along a line parallel to the $z$-axis (a) $2 r / T=0.25$, (b) $2 r / T=0.45$, (c) $2 r / T=0.55$, and (d) $2 r / T=0.65$.

Due to the dependency of the apparent viscosity of the pseudoplastic fluids on the shear rate within the stirred system, viscosity contour plots, both horizontally and vertically, were generated by the CFD model at $R_{n}=8\left(N_{c}=240 \mathrm{rpm}, N_{a}=30 \mathrm{rpm}\right)$ and are presented in Figure (5.3-10). It can be noticed from this figure that the apparent fluid viscosity inside the stirred tank was more uniform when the DSAC mixer was employed. However, the use of the DPAC mixer generated the regions with the low viscosity (higher shear rate) near the central impellers with stagnant or/and dead regions above the upper impeller, below the lower impeller, and close to the vessel wall. 
(a)

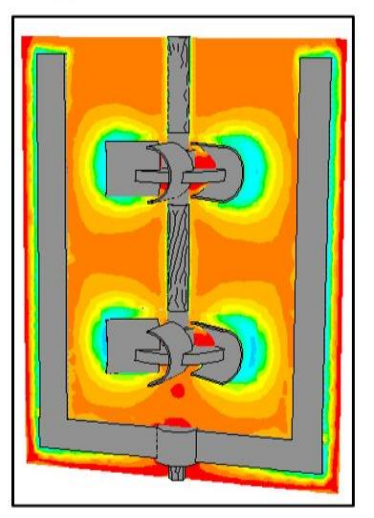

(b)

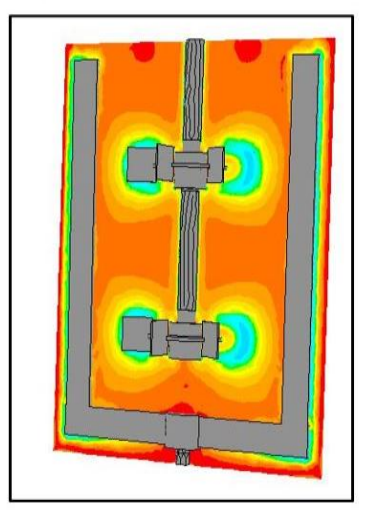

(c)
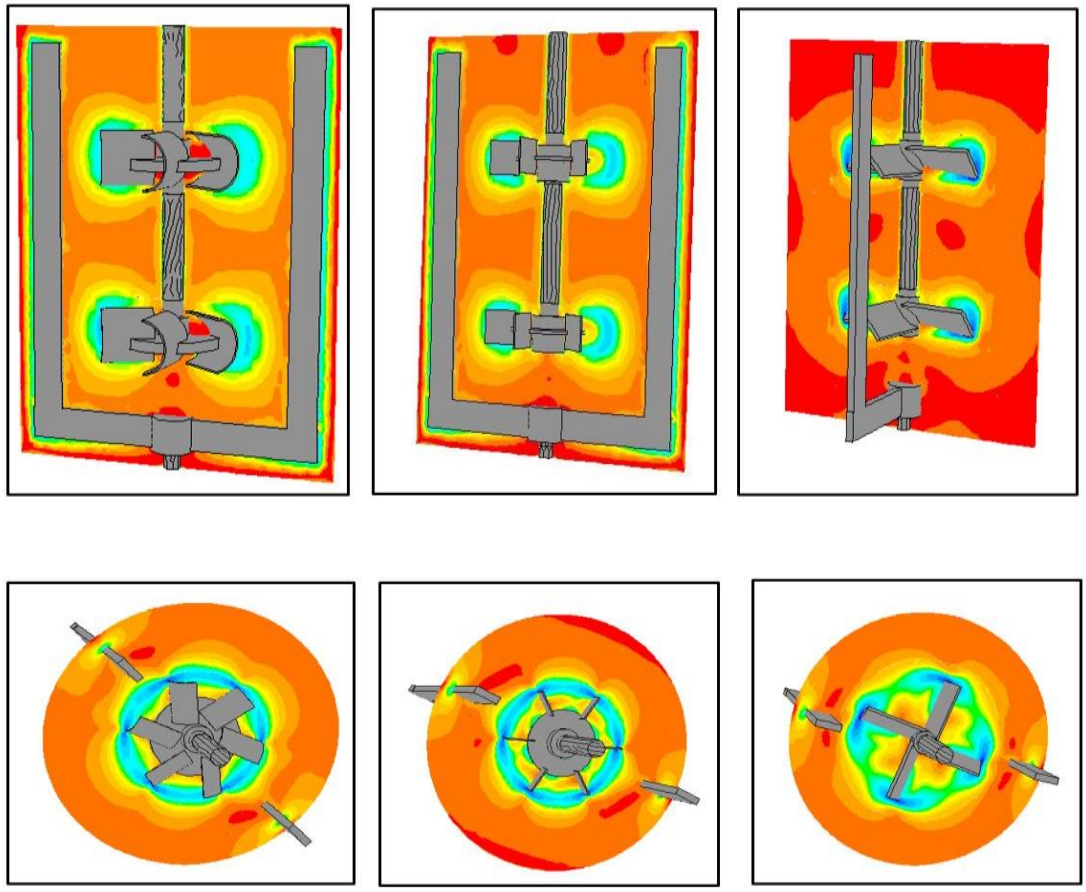

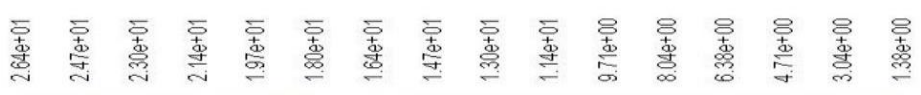

Figure (5.3-10). Vertical and horizontal viscosity $(\mathrm{kg} / \mathrm{m} . \mathrm{s})$ contour plots at $R_{n}=8\left(N_{c}=\right.$ $240 \mathrm{rpm}$ and $N_{a}=30 \mathrm{rpm}$ ) and $1.5 \%$ xanthan gum solution: (a) double Scaba-anchor coaxial (DSAC) mixer, (b) double Rushton-anchor coaxial (DRAC) mixer, and (c) double pitched blade turbine(DPAC) mixer.

\subsubsection{Power and Flow Numbers of the Coaxial Mixers}

To compare the performances of the three coaxial mixers utilized in this work, the power and flow numbers were determined for these mixers. The power numbers were calculated using the following equation (Pakzad et al., 2013b):

$$
N_{p(\text { coaxial })}=\frac{P_{t o t}}{\rho\left(N_{c}+f_{p(a)} N_{a}\right)^{3} D_{c}^{5}}
$$




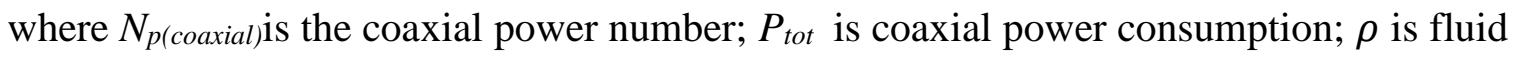
density; $N_{c}$ is central impeller rotational speed; $N_{a}$ is anchor rotational speed; $D_{c}$ is central impeller diameter; and $f_{p(a)}$ is anchor power fraction.

To calculate the flow numbers, first the impeller pumping rates $(Q)$ for each set of the double central impellers were calculated as follows (Pakzad et al., 2013d):

$$
\begin{aligned}
& Q_{(\text {Scaba, Rushton })}=\pi D \int_{-z}^{Z} \bar{V}_{r} d z \\
& Q(P B T)=2 \pi \int_{h u b}^{D / 2} \bar{V}_{a} r d r
\end{aligned}
$$

Then, based on the calculated pumping rates, the flow numbers were obtained using Equation (5.3-7) (Pakzad et al 2013 e):

$$
N_{Q}=\frac{Q}{N D^{3}}
$$

The power and flow numbers for the DSAC, DRAC, and DPAC mixers at $R_{n}=8\left(N_{c}=\right.$ $240 \mathrm{rpm}, N_{a}=30 \mathrm{rpm}$ ) are listed in Table (5.3-6). It can be seen that for three studied coaxial mixers, the flow number of the upper central impeller was higher than that for the lower central impeller. However, the power number of the upper central impeller was slightly lower than that for the lower central impeller. A similar finding has been reported in the mixing of Newtonian fluids by multiple impellers without anchor in turbulent mode by Rutherford et al. (1996) and Mishra et al. (1994). Based on the calculated numbers listed in Table (5.3-6), it can be concluded that the DSAC mixer is more efficient with respect to the pumping rate at the operating conditions studied in this paper. 
Table (5.3-6). The power number and flow number of the three coaxial mixers.

\begin{tabular}{ccccc}
\hline Coaxial type & $N_{P u}$ & $N_{P l}$ & $N_{Q u}$ & $N_{Q l}$ \\
\hline DSAC & 6.94 & 6.96 & 0.313 & 0.302 \\
DRAC & 6.66 & 6.68 & 0.353 & 0.242 \\
DPAC & 5.40 & 5.42 & 0.218 & 0.213 \\
\hline
\end{tabular}

\subsubsection{Mixing Time}

The mixing time measurements were reported in Figure (5.3-3) for three coaxial mixers through the validated CFD model for the xanthan gum solution. These data show that the DSAC mixer had the lowest mixing time compared with the others. For a more in-depth analysis, the normalized mixing time curves at $R e=173$ for three coaxial mixer configurations were obtained at two monitoring points: one between two central impellers and the second one between the lower central impeller and anchor as shown in Figure (5.3-11), respectively. As expected the DSAC and DRAC mixing systems showed better and faster tracer distribution. This was due to the formation of circulation loops, which eliminated the formation of the segregated zones (Bonnot et al., 2007; Farhat et al., 2007) inside the tank when these two coaxial impellers were utilized. However, these mixing curves for the DPAC mixer show two plateau sections at two monitoring points. The presence of these plateau sections was due to the axial flow compartmentalization, which resulted in the formation of the segregated zones inside the agitated tank. 
(a)

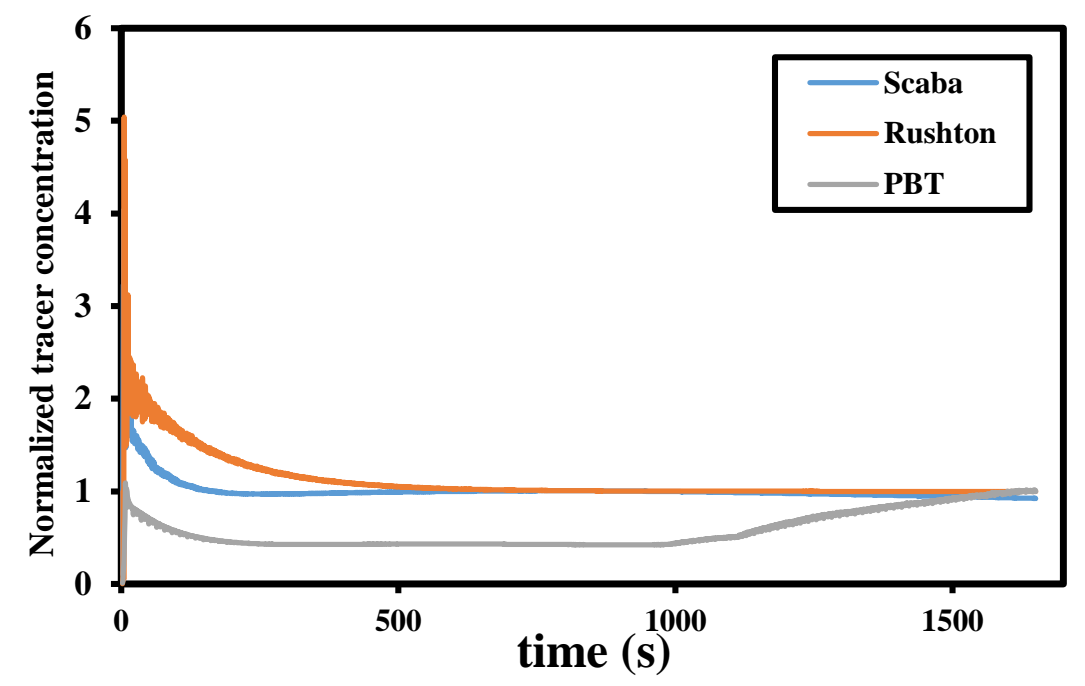

(b)

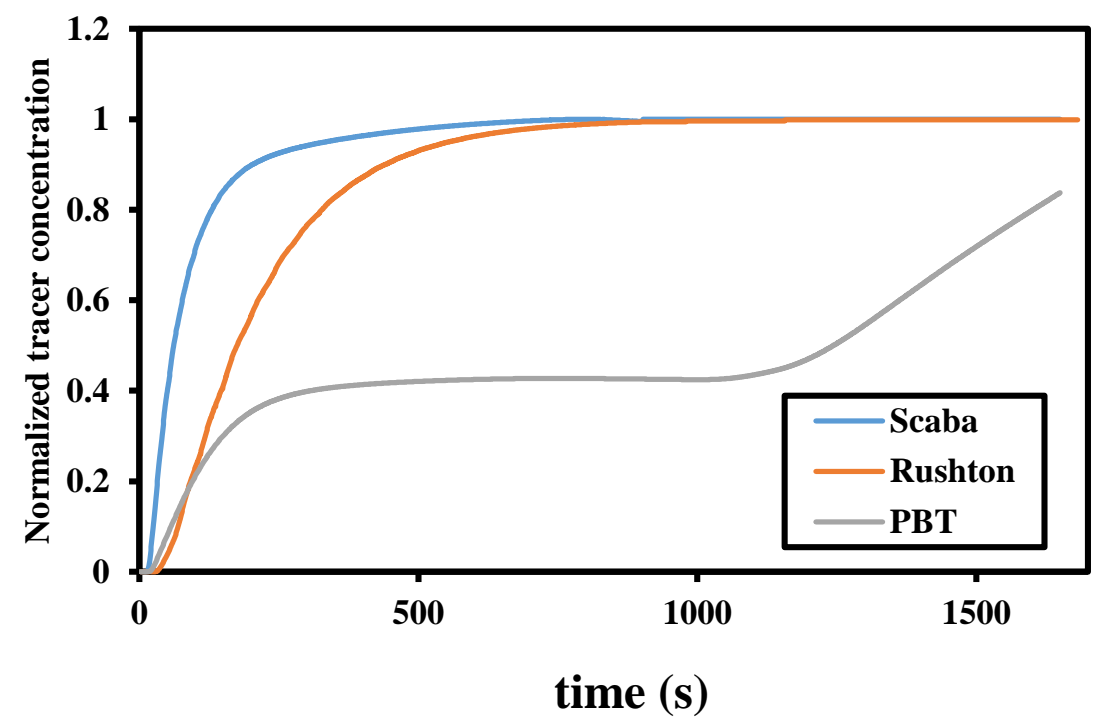

Figure (5.3-11). Normalized tracer concentration verses time at $R_{n}=8\left(N_{c}=240 \mathrm{rpm}\right.$ and $N_{a}=30 \mathrm{rpm}$ ) and 1.5\% xanthan gum solution: (a) between two central impellers at position $(0.13,-0.03,0.25 \mathrm{~m})$ and $(\mathbf{b})$ under the lower impeller at position $(0.00,0.00$, $0.12 \mathrm{~m})$. 


\subsubsection{Mixing Efficiency}

The following dimensionless number proposed by Pakzad et al. (2013c) was used to evaluate the efficiency of the three coaxial mixers analyzed in this paper:

$$
1 / \pi_{1}=\frac{t_{m}{ }^{2} P_{t o t}}{\eta V}
$$

where $\eta$ is apparent viscosity of non-Newtonian fluid; $t_{m}$ is mixing time; $P_{t o t}$ is total power consumption; and $V$ presents fluid volume. A shorter mixing time can be achieved with a lower power consumption for an effective impeller. Thus, the value of $1 / \pi_{1}$ is lower for a more efficient mixer. Figure (5.3-12) shows $1 / \pi_{1}$ versus the speed ratio for the DSAC, DRAC, and DPAC mixers. These results revealed that the DSAC mixer was more effective for the agitation of non-Newtonian yield-pseudoplastic fluids than the other two mixers.

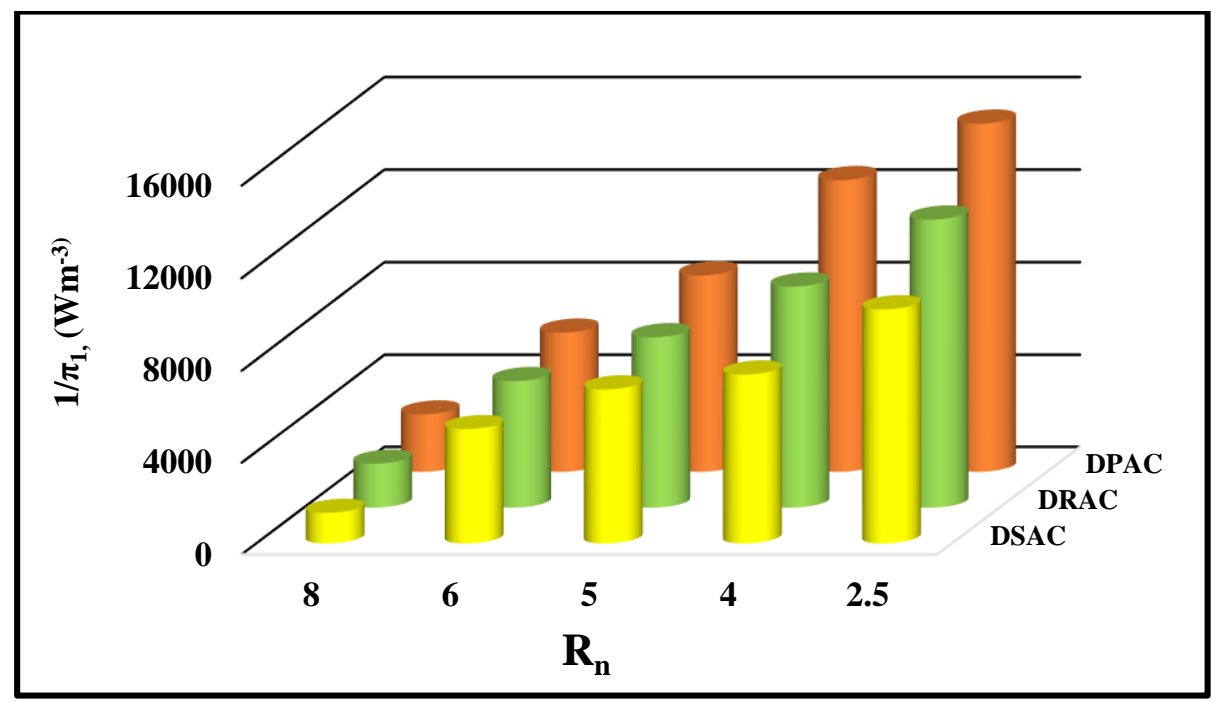

Figure (5.3-12). $\left(1 / \pi_{l}\right)$ verses speed ratio for the double Scaba-anchor coaxial (DSAC), double Rushton-anchor coaxial (DRAC), and double pitched blade turbine-anchor (DPAC) mixers at $1.5 \%$ xanthan gum solution. 


\subsubsection{Conclusion}

The performances of the double Scaba-anchor coaxial (DSAC), double Rushton-anchor coaxial (DRAC), and double pitched blade-anchor coaxial (DPAC) mixers in the agitation of yield pseudoplastic fluids in the laminar-transitional regime in the co-rotating mode were analyzed and compared. The assessment was performed both numerically and experimentally through computational fluid dynamics (CFD), electrical resistance tomography (ERT), and design of experiment (DOE) combined with the response surface methodology (RSM). The collected data were utilized to evaluate the hydrodynamic performances of these three coaxial mixers with respect to the mixing time, power consumption, generated fluid flow domain, and mixing energy. RSM plots demonstrated that the DSAC mixer had the lowest mixing time among the three coaxial mixers within the experimental range studied in this paper. An analysis of the normalized mixing time curves for three coaxial mixers showed that the DSAC and DRAC mixing systems showed better and faster tracer distribution. This was due to the formation of circulation loops, which eliminated the formation of the segregated zones inside the tank when these two coaxial impellers were utilized. However, these mixing curves for the DPAC mixer showed two plateau sections at two monitoring points. The presence of these plateau sections was due to the axial flow compartmentalization, which resulted in the formation of the segregated zones inside the agitated tank. The simulated flow domain by the CFD model proved that both DSAC and DRAC mixers generated similar stable parallel flow patterns at the impeller spacing equal to $T / 2$ while the flow pattern created by the DPAC mixer was unstable resulting in less efficient mixing operation. Further analysis to the velocity profiles revealed that DSAC mixer created a more uniform velocity distribution throughout the tank compared to the other two coaxial mixers. Furthermore, it was found 
that the DRAC mixer induced the highest shear rate with a maximum magnitude close to the upper and lower central impellers. However, the shear rate profile generated by the DSAC mixer in the radial direction was more consistent than those by the other two mixers. To characterize these three coaxial mixers, the flow numbers were also calculated and the results showed that the DSAC mixer was more effective with respect to the pumping rate at the operating conditions studied in this paper. Finally, the mixing energy versus the speed ratio was plotted for three coaxial mixers and these data revealed that the DSAC mixer was more efficient for the agitation of non-Newtonian yieldpseudoplastic fluids than the other two coaxial mixers. 


\subsection{An Investigation on the Effect of the Impeller Spacing on the Flow Field Generated by the Coaxial Mixing System Composed of Double Central Impellers and an Anchor in the Agitation of Yield- Pseudoplastic Fluids}

\subsubsection{Introduction}

The main focus of the present study was to explore the influence of the impeller spacing on the three-dimensional laminar-transitional flow field generated in the mixing of the xanthan gum solution (a yield pseudoplastic fluid) with a coaxial mixing system composed of double Scaba impellers in combination with an anchor [Figure (5.4-1]. To evaluate the influence of the rotation mode, the experiments were carried out in both corotating and counter-rotating regime. The 3-D numerical simulations of the flow domain were performed through the computational fluid dynamics (CFD). The measurement of the mixing times was performed by the non-intrusive electrical resistance tomography (ERT) technique. The velocity profiles, power numbers, flow numbers, mixing times, and pumping effectiveness were computed through the validated CFD model.
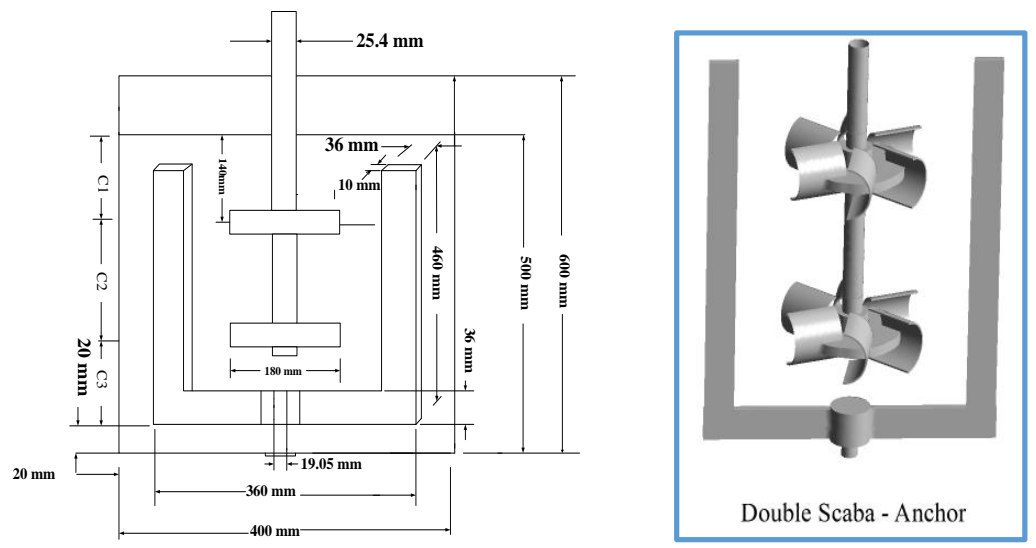

Figure (5.4-1). Confirmation coaxial in this study.

The submergence was set to $C_{1}=0.140 \mathrm{~m}$, and the separation between impellers $\left(C_{2}\right)$, and off-bottomed clearance $\left(C_{3}\right)$ from the base of the vessel were varied as shown in 
Table (5.4-1). The experiments were carried out at the generalized Reynolds numbers (Re) of 173 for the co-rotating regime and 183 for the counter-rotating regime. These Reynolds numbers were calculated using Pakzad et al. (2013b) correlation for the rotational speeds of $N_{s}=240 \mathrm{rpm}$ and $N_{a}=30 \mathrm{rpm}$ for the Scaba and anchor impellers, respectively.

Table (5.4-1). Configurations of the coaxial mixer.

\begin{tabular}{cccc}
\hline Double impeller & $\begin{array}{c}\text { Upper impeller } \\
\text { submergence } \\
\text { Scaba-Scaba }\end{array}$ & Impeller spacing & $\begin{array}{c}\text { Lower Impeller } \\
\text { clearance }\end{array}$ \\
\hline SS1 & 0.140 & $\mathrm{C}_{2}(\mathrm{~m})$ & $\mathrm{C}_{3}(\mathrm{~m})$ \\
SS2 & 0.140 & 0.090 & 0.270 \\
SS3 & 0.140 & 0.127 & 0.233 \\
SS4 & 0.140 & 0.175 & 0.185 \\
SS5 & 0.140 & 0.200 & 0.160 \\
SS6 & 0.140 & 0.226 & 0.134 \\
\hline
\end{tabular}

The CFD model was developed and validated by comparing the experimentally determined specific power drawn and mixing time data with the computed values attained by the CFD model for different configurations of the coaxial mixer listed in Table (5.41). This comparison showed a good agreement between the calculated data and measured results with the standard deviations less than 5\% as presented in Table (5.4-2). The validated CFD model was then used to obtain further information to assess the performances of different configurations of the coaxial mixer. 
Table (5.4-2). Computed and measured power consumption and mixing time for different configurations of coaxial mixer at $R_{n}=8\left(N_{a}=30 \mathrm{rpm}\right.$ and $\left.N_{s}=240 \mathrm{rpm}\right)$ and $1.5 \%$ xanthan gum solution in the co-rotating mode.

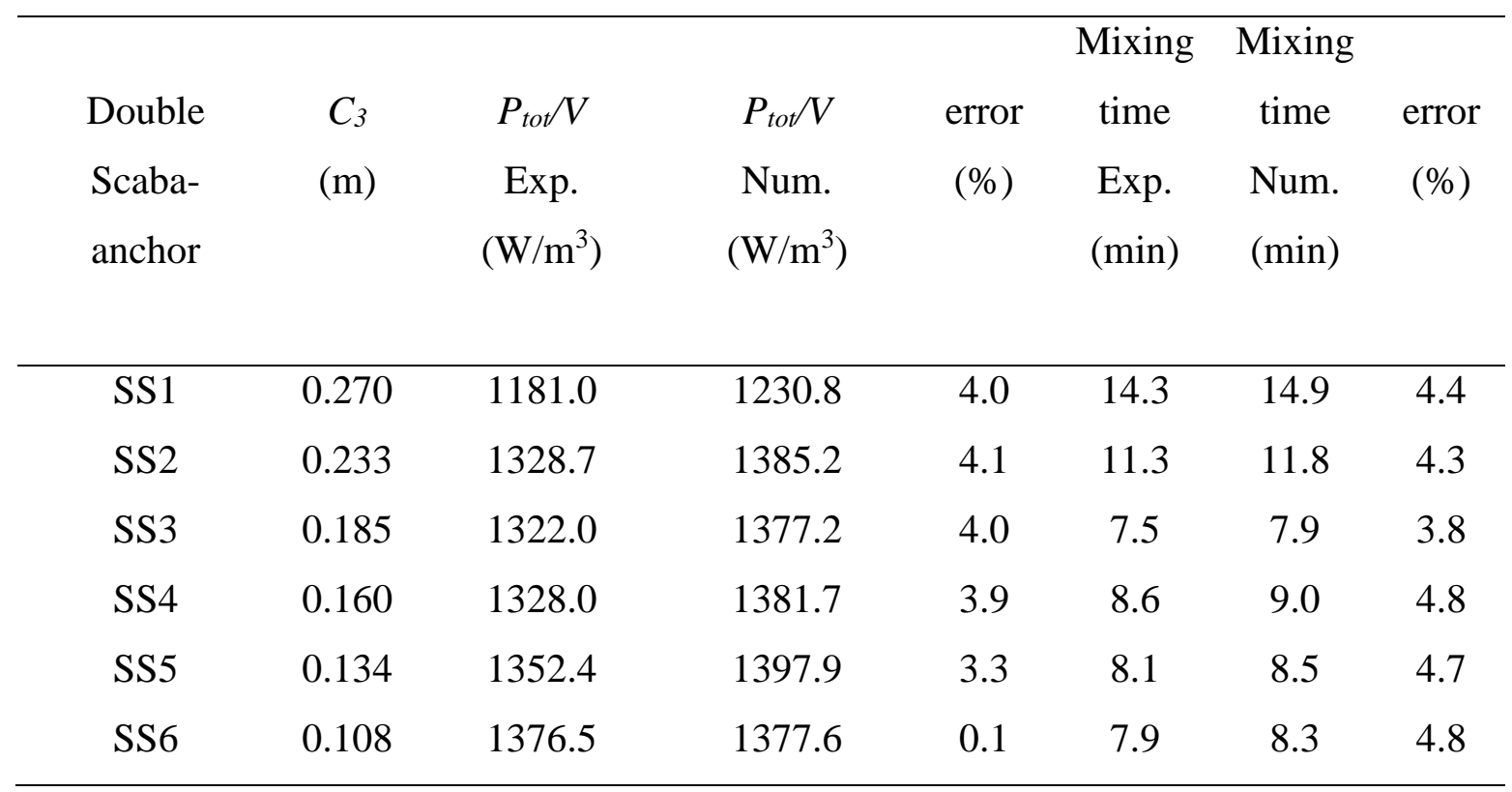

\subsubsection{Results and Discussion}

\subsubsection{Power Number, Flow Number, Pumping Effectiveness, Mixing Time, and Mixing Efficiency}

Power number, flow number, mixing time, and pumping effectiveness were employed to assess the performances of the different configurations of the coaxial mixer. Correlation developed by Pakzad et al. (2013b) was adopted to translate power consumption to power number for both co-rotating and counter-rotating regimes:

$$
N_{p(\text { coaxial })}=\frac{P_{t o t}}{\rho\left(N_{s}+f_{p(a)} N_{a}\right)^{3} D_{s}^{5}}
$$


In order to calculate the flow numbers of the different configurations of the coaxial mixing system, pumping capacity $\left(Q_{r}\right)$ of the radial Scaba impeller for each configuration was calculated by integrating the mean average radial velocity on a vertical plane at the tip of the impeller $\left(r=R_{i}\right)$ from the lower edge $(-z=0.03 \mathrm{~m})$ to the upper edge $(+z=0.03$ m) of the impeller (Pakzad et al., 2013a) as follows:

$$
Q_{r(\text { Scaba })}=\pi D_{s} \int_{-z}^{z} \bar{V}_{r} d z
$$

Then, the flow number and the pumping effectiveness for each coaxial mixing configuration $\left(\eta_{E}\right)$ was defined by (Mishra and Joshi, 1994):

$$
\begin{aligned}
& N_{Q}=\frac{Q_{r}}{N D_{S}{ }^{3}} \\
& \eta_{E}=\frac{N_{Q}}{N_{P}} \times 100
\end{aligned}
$$

To compare the effect of the rotating regime on the hydrodynamic performance of the coaxial agitated mixer, the power numbers, flow numbers, mixing times, and pumping effectiveness for various configurations of the double Scaba-anchor coaxial agitated mixer for both rotating modes at the same operating conditions $\left(R_{n}=8\right.$ and $1.5 \%$ xanthan gum solution) were calculated and are summarized in Tables (5.4-3) and (5.4-4). These results indicate that the total flow numbers for all configurations (SS1, SS2, SS3, SS4, SS5 and SS6) in the co-rotating regime were higher than those in the counter-rotating regime. In terms of power number, it was found that the total power numbers in the counter-rotating mode for all coaxial mixing configurations were higher than those in the co-rotating mode. This was due to this fact that in the co-rotating mode the double Scaba impeller blades induce flow that drags anchor impeller blade resulting in the decreased 
power drawn compared to the counter-rotating one. This is in accordance with the study conducted by Rudolph et al. (2009). The results listed in Tables (5.34-3) and (5.4-4) also show that the power number for the SS1 configuration was the lowest one while it was the highest for the SS6 configuration in both rotating regimes. Moreover, the mixing time measurements were carried out for different coaxial mixing configurations operated in the co-rotating and counter-rotating modes. It can be noted that the mixing times in the counter-rotating mode were higher than those in the co-rotating one at the same operating conditions. The pumping effectiveness values presented in Tables (5.4-3) and (5.4-4) for all configurations show that the coaxial stirrers operated in the co-rotating regime were more efficient than those operated in the counter-rotating regime. Therefore, in the remaining sections of this paper, we focused on the agitation of non-Newtonian yieldpseudoplastic fluids with the various configurations of the coaxial mixing system in the co-rotating mode.

The data listed in Table (5.4-3) show that the flow numbers of the lower and the upper impellers for different coaxial mixing configurations were close to each other. It can be seen that the flow numbers of the upper impeller for the SS3, SS4, SS5, and SS6 configurations were slightly higher than those of the lower impeller. Accordingly, the highest total flow numbers were attained for the SS3 $\left(N_{Q}=0.85\right)$ and SS4 $\left(N_{Q}=0.84\right)$ configurations. Moreover, it was found that the overall power numbers for various configurations changed as the impeller spacing was increased from $C_{2}=0.090 \mathrm{~m}(0.18$ $H)$ to $C_{2}=0.252 \mathrm{~m}(0.50 H)$. The lowest power number and the highest mixing time were recorded for the SS1 configuration. The lowest mixing times were achieved by the SS3 and SS6 configurations while the power number of the SS6 coaxial mixer $\left(N_{P}=7.22\right)$ 
mode was higher than that of the SS3 coaxial mixer $\left(N_{P}=6.34\right)$. Furthermore, the calculated pumping effectiveness for each configuration showed that the SS3 configuration had the highest pumping efficiency compared to the others.

Table (5.4-3). Power number, flow number, pumping effectiveness and mixing time for different configurations of coaxial mixer at $R_{n}=8\left(N a=30 \mathrm{rpm}\right.$ and $\left.N_{s}=240 \mathrm{rpm}\right)$ and $1.5 \%$ xanthan gum solution in the co-rotating mode.

\begin{tabular}{|c|c|c|c|c|c|c|}
\hline $\begin{array}{l}\text { Double } \\
\text { Scaba- } \\
\text { anchor }\end{array}$ & $\begin{array}{l}\text { Power } \\
\text { Number } \\
\quad\left(N_{p}\right)\end{array}$ & $\begin{array}{c}\text { Flow } \\
\text { number of } \\
\text { upper } \\
\text { impeller } \\
N_{Q u}\end{array}$ & $\begin{array}{c}\text { Flow } \\
\text { number of } \\
\text { lower } \\
\text { impeller } \\
N_{Q l}\end{array}$ & $\begin{array}{c}\text { Total } \\
\text { flow } \\
\text { number } \\
\mathrm{N}_{\mathrm{Qt}}\end{array}$ & $\begin{array}{c}\text { Mixing } \\
\text { time } \\
(\min )\end{array}$ & $\begin{array}{c}\text { Pumping } \\
\text { effectiveness } \\
\left(N_{Q} / N_{P}\right) \times 100\end{array}$ \\
\hline SS1 & 6.19 & 0.31 & 0.32 & 0.63 & 14.32 & 10.20 \\
\hline $\mathrm{SS} 2$ & 6.55 & 0.35 & 0.36 & 0.71 & 11.29 & 10.80 \\
\hline SS3 & 6.34 & 0.44 & 0.41 & 0.85 & 7.58 & 13.20 \\
\hline SS4 & 6.80 & 0.40 & 0.37 & 0.77 & 8.60 & 11.30 \\
\hline SS5 & 7.05 & 0.43 & 0.40 & 0.81 & 8.10 & 11.50 \\
\hline SS6 & 7.22 & 0.43 & 0.41 & 0.84 & 7.90 & 11.70 \\
\hline
\end{tabular}


Table (5.4-4). Power number, flow number, pumping effectiveness and mixing time for different configuration of coaxial mixer at $R_{n}=8\left(N_{a}=31 \mathrm{rpm}\right.$ and $\left.N_{s}=240 \mathrm{rpm}\right)$ and $1.0 \%$ xanthan gum solution in the counter-rotating mode.

\begin{tabular}{|c|c|c|c|c|c|c|}
\hline $\begin{array}{l}\text { Double } \\
\text { Scaba- } \\
\text { anchor }\end{array}$ & $\begin{array}{c}\text { Power } \\
\text { Number } \\
\quad\left(N_{p}\right)\end{array}$ & $\begin{array}{c}\text { Flow } \\
\text { number of } \\
\text { upper } \\
\text { impeller } \\
N_{Q u}\end{array}$ & $\begin{array}{c}\text { Flow } \\
\text { number of } \\
\text { lower } \\
\text { impeller } \\
N_{Q l}\end{array}$ & $\begin{array}{c}\text { Total } \\
\text { flow } \\
\text { number } \\
N_{Q t}\end{array}$ & $\begin{array}{l}\text { Mixing } \\
\text { time } \\
\text { (min) }\end{array}$ & $\begin{array}{c}\text { Pumping } \\
\text { effectiveness } \\
\left(N_{Q} / N_{P}\right) \times 100\end{array}$ \\
\hline SS1 & 7.17 & 0.31 & 0.27 & 0.61 & 17.30 & 8.00 \\
\hline $\mathrm{SS} 2$ & 8.86 & 0.36 & 0.36 & 0.72 & 13.70 & 8.10 \\
\hline SS3 & 8.79 & 0.38 & 0.37 & 0.75 & 8.80 & 8.50 \\
\hline SS4 & 8.85 & 0.36 & 0.37 & 0.73 & 10.50 & 8.30 \\
\hline SS5 & 8.88 & 0.40 & 0.38 & 0.78 & 9.70 & 8.70 \\
\hline SS6 & 9.02 & 0.42 & 0.39 & 0.81 & 9.20 & 8.90 \\
\hline
\end{tabular}

In order to determine the optimal impeller spacing, the power number and mixing time were plotted as the function of the impeller spacing $\left(C_{2}\right)$ at the speed ratio $R_{n}=8\left(N_{s}=\right.$ $240 \mathrm{rpm}$ and $N_{a}=30 \mathrm{rpm}$ ) and $R e=172$ in the co-rotating regime and the results are depicted in Figure (5.4-2). These data clearly demonstrate that the mixing time decreased with an increasing impeller spacing from 0.09 to $0.175 \mathrm{~m}$, then increased when the impeller spacing increased from 0.175 to $0.200 \mathrm{~m}$, and again decreased with increasing the impeller spacing from 0.200 to $0.252 \mathrm{~m}$ in the co-rotating regime. It can be noted that the mixing time of the SS3 configuration was the lowest among all cases. Furthermore, the power number of the coaxial mixing system increased as the impeller spacing was increased from 0.090 to $0.127 \mathrm{~m}$ and then decreased by increasing the impeller spacing 
from 0.127 to $0.175 \mathrm{~m}$. However, the power number again increased when the impeller spacing was increased from 0.175 to $0.252 \mathrm{~m}$. The highest power number of 7.22 was obtained by the SS6 configuration while the lowest power number $\left(N_{p}=6.19\right)$ was achieved by the SS1 configuration. Overall, the results presented in Figure (5.4-2) demonstrate that the optimal impeller spacing was 0.175 , which was attained for the SS3 configuration.

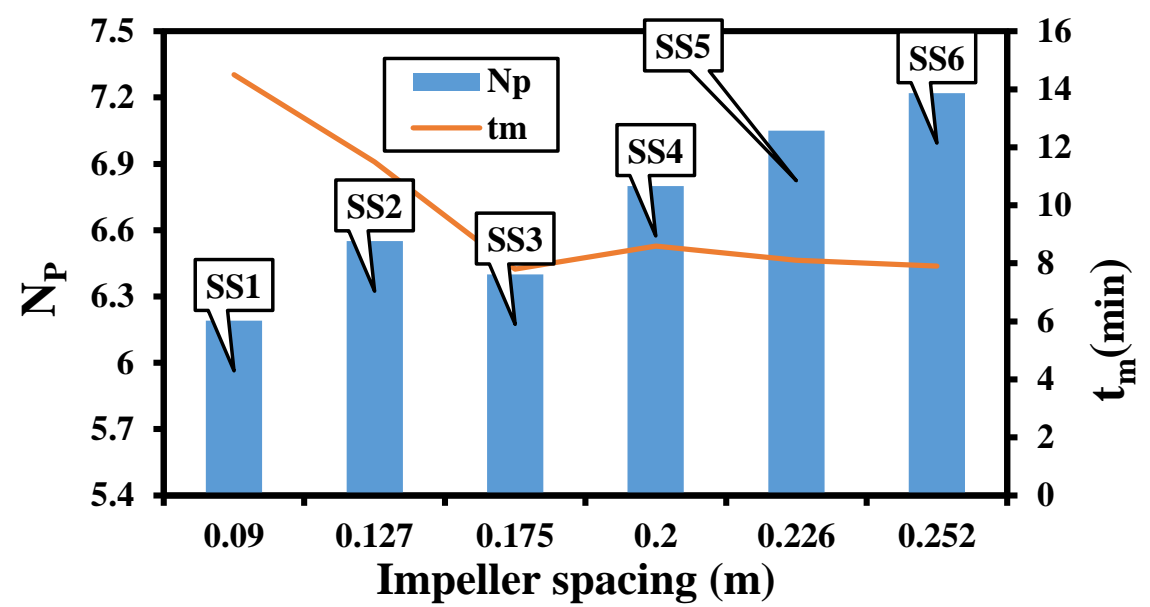

Figure (5.4-2). Power number and mixing time versus the impeller spacing (m) for different coaxial mixing configurations at $R_{n}=8\left(N_{a}=30 \mathrm{rpm}\right.$ and $\left.N_{s}=240 \mathrm{rpm}\right)$ and $1.5 \%$ xanthan gum solution in the co-rotating mode. 
In order to obtain the mixing efficiency, the energy consumption $\left(P . t_{m}\right)$, which is the product of the power consumption and mixing time (Mishra et al., 1994), was calculated for various coaxial mixing configurations employed in this study. Figure (5.4-3) shows the energy consumption as a function of the impeller spacing $\left(C_{2}\right)$ at the speed ratio of $R_{n}$ $=8\left(N_{s}=240 \mathrm{rpm}\right.$ and $\left.N_{a}=30 \mathrm{rpm}\right)$ and $R e=172$ in the co-rotating mode. It was found that the SS3 configuration was the most efficient system with the minimum energy consumption among the investigated configurations.

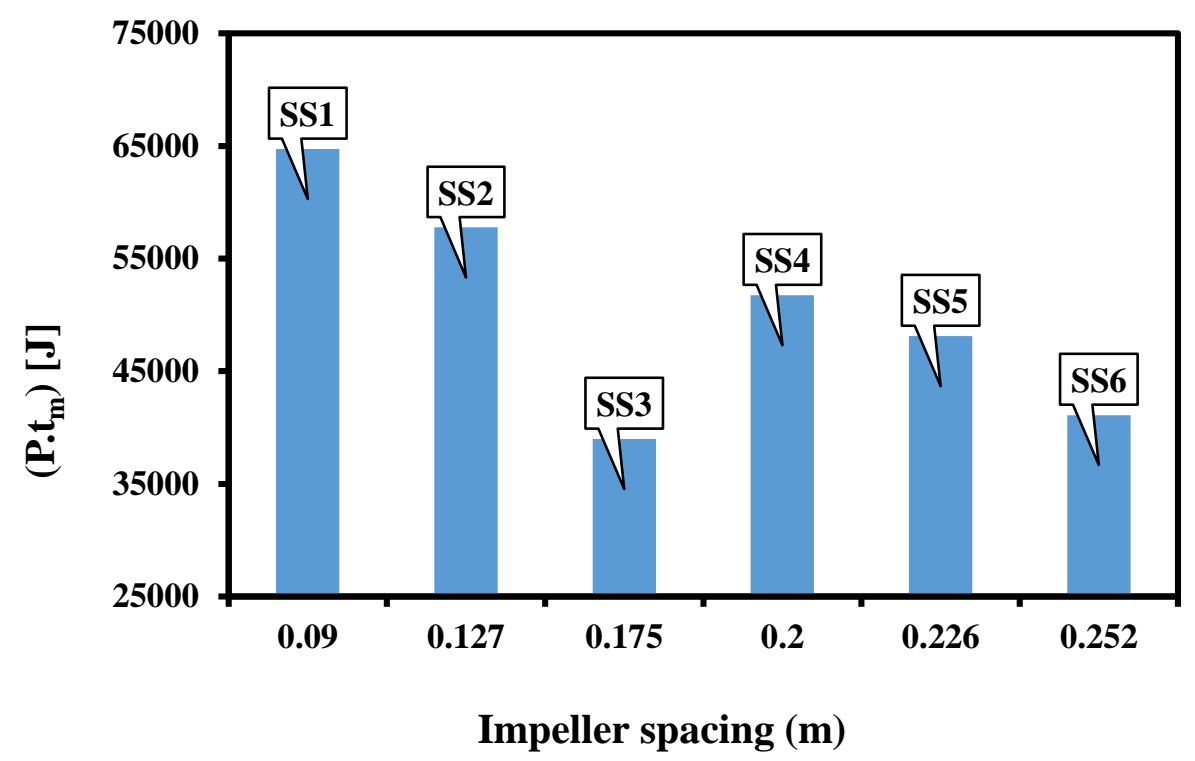

Figure (5.4-3). Energy consumption as a function of the impeller spacing for different coaxial mixing configurations at $R_{n}=8\left(N_{a}=30 \mathrm{rpm}\right.$ and $\left.N_{s}=240 \mathrm{rpm}\right)$ and $1.5 \%$ xanthan gum solution in the co-rotating mode.

\subsubsection{Flow Pattern}

The purpose of this section is to discuss the influence of the impeller spacing $\left(C_{2}\right)$ on the 3-D laminar flow generated by various configurations of the coaxial mixer in the co- 
rotating mode. To compare and analyze the effect of the impeller spacing $\left(C_{2}\right)$ on the mixing mechanism and flow pattern, both qualitative and quantitative analysis were adopted.

Figure (5.4-4) depicts the velocity contour plots calculated at $R_{n}=8\left(N_{s}=240 \mathrm{rpm}\right.$ and $N_{a}=30 \mathrm{rpm}$ ) and $R e=172$ in the co-rotating mode. As can be noticed in Figure (5.4-4 a), for the SS1 configuration with an upper impeller submergence of $C_{1}=0.140 \mathrm{~m}$, an impeller spacing of $C_{2}=0.090 \mathrm{~m}$, and an off-bottom clearance of $C_{3}=0.270 \mathrm{~m}$, a cavern (well-mixed zone) around the double Scaba impellers with the dead or near stagnant regions at the rest of the vessel, particularly under the double central impellers, were observed. It can be noted that the size and position of the well- mixed zone were changed when the impeller spacing $\left(C_{2}\right)$ was increased from $0.090 \mathrm{~m}$ to $0.252 \mathrm{~m}$. It can be concluded that by increasing the distance between two central impellers the flow was intensified in both radial and axial directions resulting in the elimination or reduction of the dead zones within the mixing tank. 
(a)

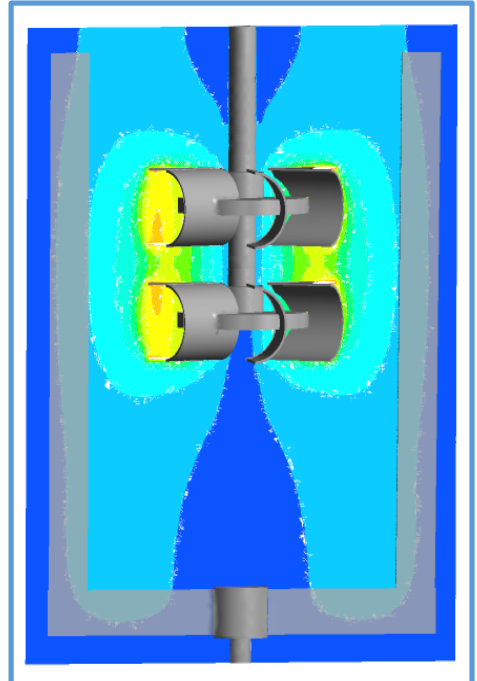

(d)

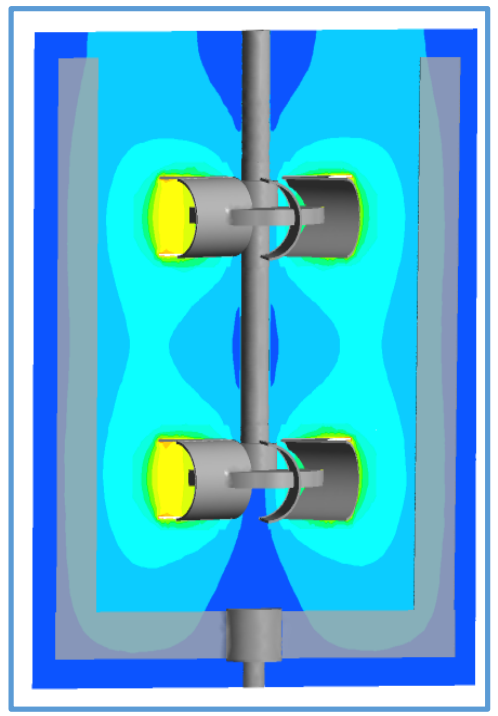

(b)

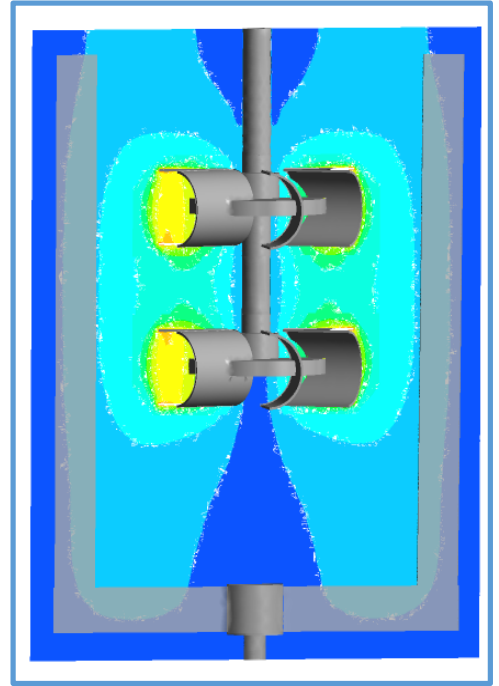

(e)

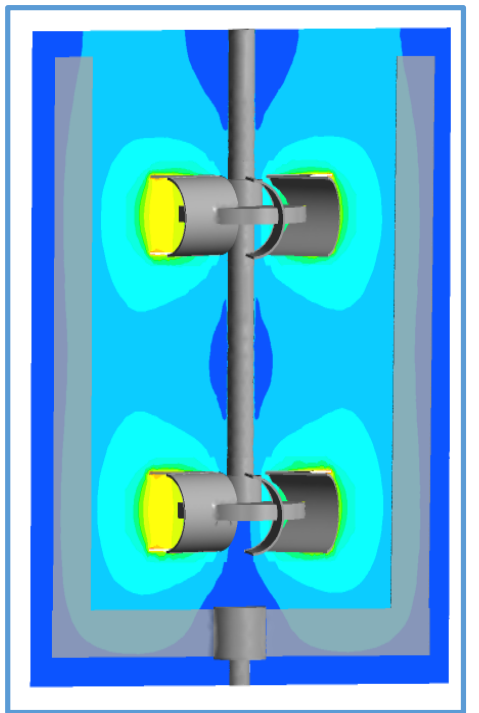

(c)

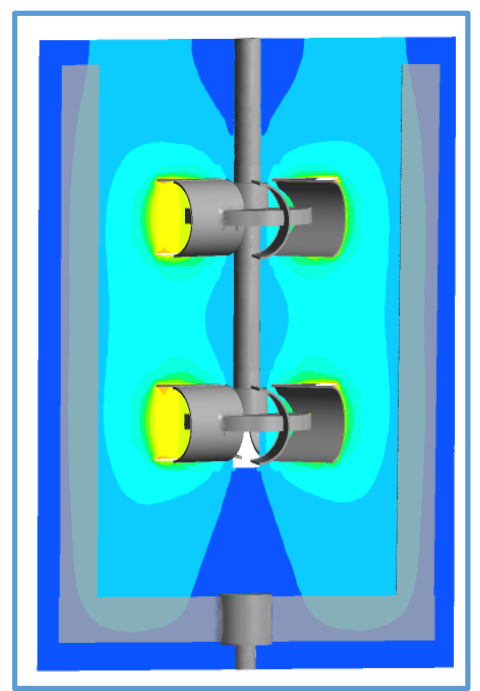

(f)

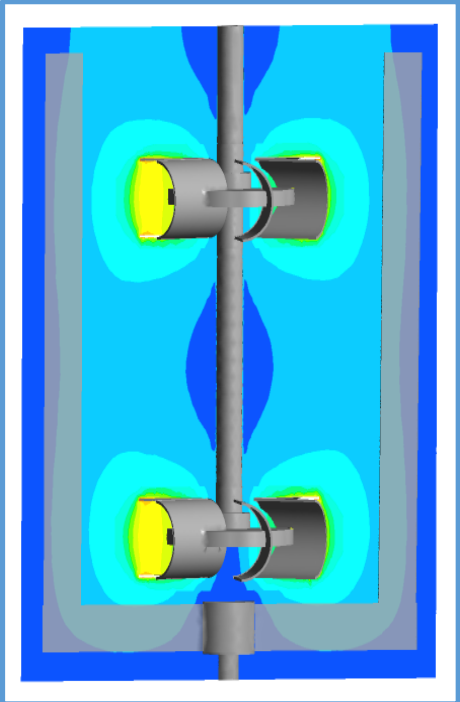

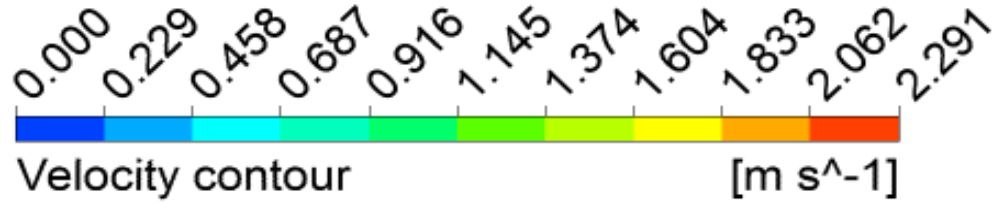

Figure (5.4-4) Velocity $(\mathrm{m} / \mathrm{s})$ contour plots generated by different coaxial mixing configurations at $R_{n}=8\left(N_{a}=30 \mathrm{rpm}\right.$ and $\left.N_{s}=240 \mathrm{rpm}\right)$ and $1.5 \%$ xanthan gum solution in the co-rotating mode: (a) SS1, (b) SS2, (c) SS3, (d) SS4, (e) SS5, and (f) SS6. 
To conduct an in-depth analysis of the results shown in Figure (5.4-4), the velocity vector and streamline plots are depicted in Figures (5.4-5) and (5.4-6). One clearly notices that different kinds of flow patterns were generated as the spacing $\left(C_{2}\right)$ between two central impellers changed. When the impeller spacing $(C 2)$ was set to $0.090 \mathrm{~m}(0.18 H)$, an almost straight-line orientation was followed by the impeller streams toward one another and then they merged at an elevation midway between two impellers [Figure (5.4-5) a and Figure (5.4-6) a]. As a result, two large ring vortices were formed. This flow pattern is called "merging flow". A similar flow pattern has been reported by Rutherford et al. (1996) and Mishra and Joshi (1994) in the mixing Newtonian fluid by double Rushton impellers without anchor in turbulent regime. As it can be observed, the combination of two Scaba impellers operated as a single impeller producing only one radial outflow in the merging point. In this flow pattern, due to closeness of two impellers, a cylindrical region between two Scaba impellers promoted the low velocity zone resulting in inadequate motion, particularly for the laminar flow. Such a low velocity zone has been reported by Mishra and Joshi (1994) in the mixing of Newtonian fluid with double Rushton turbine impellers without anchor in turbulent mode.

Furthermore, Figures (5.4-5) $\mathrm{b}-(5.4-5) \mathrm{f}$ and (5.4-6) $\mathrm{b}-(5.4-6) \mathrm{f}$ show that the merging flow pattern changed gradually to the parallel flow when the distance between two central impellers $\left(C_{2}\right)$ was increased from $0.090 \mathrm{~m}(0.18 H)$ to $0.252 \mathrm{~m}(0.5 \mathrm{H})$. For the impeller spacing of $C_{2}>0.175 \mathrm{~m}(0.35 \mathrm{H})$, the fluid flows generated by the Scaba impellers did not merge and each Scaba impeller acted like a single impeller producing a radial jet in the outward direction. Close to the tank wall, each striking jet was divided into two upward and downward flows creating two circulation loops for each individual 
impeller. Therefore, the flow pattern comprised of 8 ring vortices, two above the upper Scaba impeller, four between the central impellers, and two below the lower impeller. As it can be observed, in this flow pattern, which is called "parallel flow", (Rutherford et al., 1996) streams were almost parallel to one another. Moreover, it can be noted that for the impeller spacing greater than $0.175 \mathrm{~m}(0.35 \mathrm{H})$, the flow pattern at the bottom of the vessel was affected by the rotation of the anchor impeller. It is noteworthy mention that the flow patterns created by the double central Rushton turbines without anchor in the agitation of Newtonian fluids were investigated by Rutherford et al. (1996) and Mahmoudi et al. (1991) in the turbulent regime. Three flow patterns were reported: parallel flow, merging flow, and diverging flow. However, only parallel and merging flows were observed in the mixing of yield-pseudoplastic fluids by a coaxial agitated mixer in the laminar-transitional mode. 
(a)

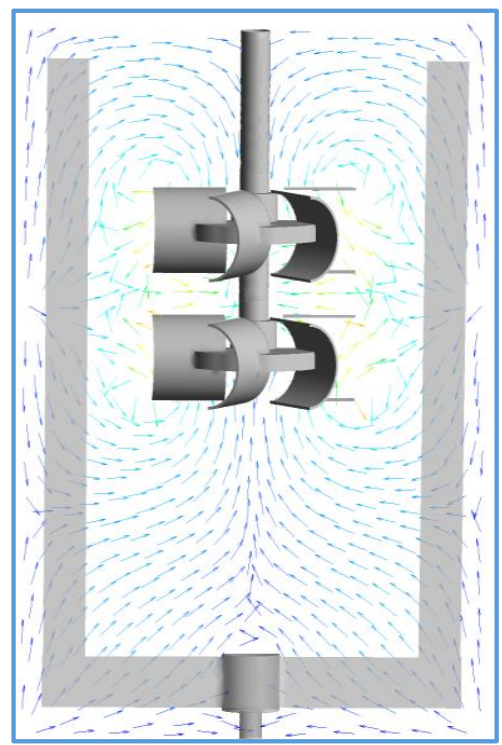

(d)

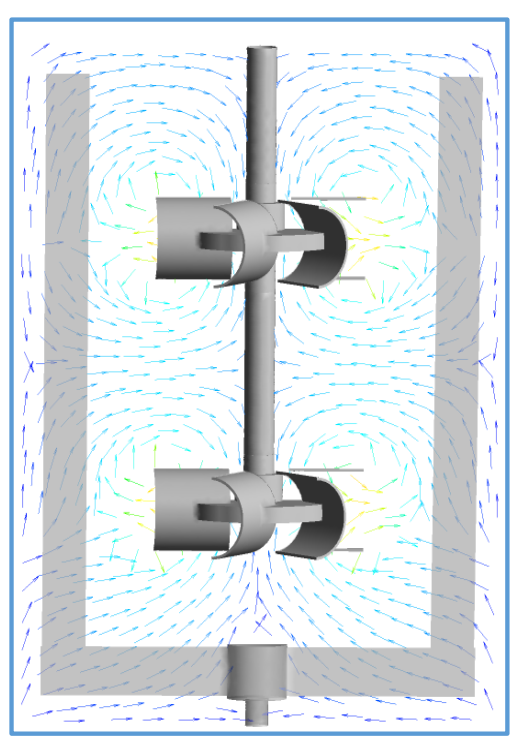

(b)

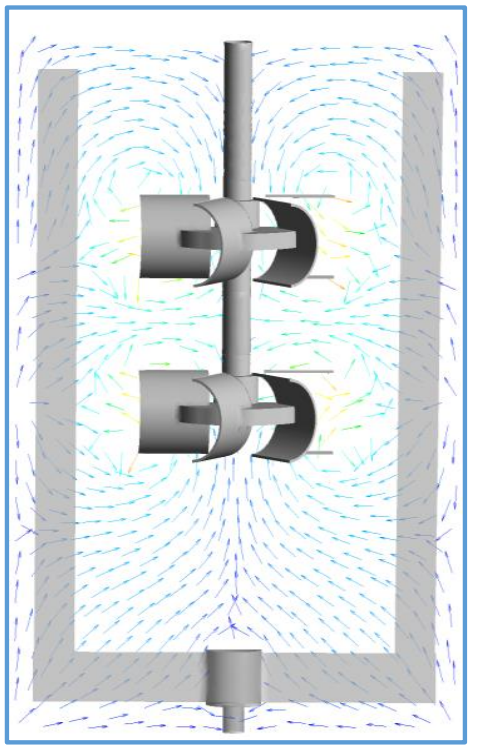

(e)

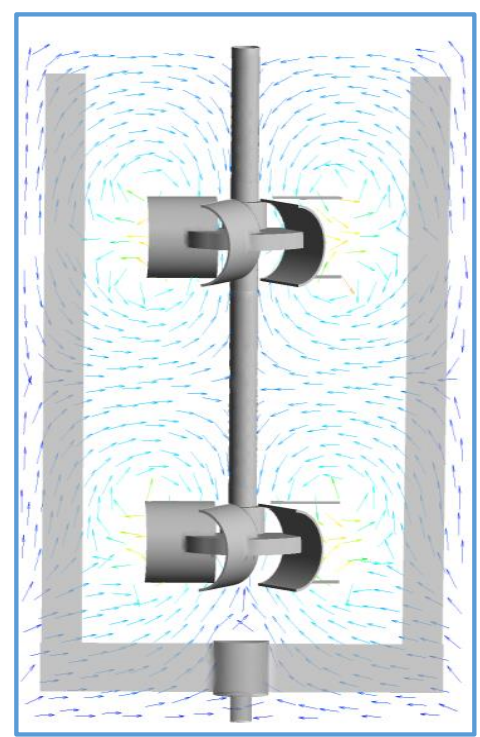

(c)

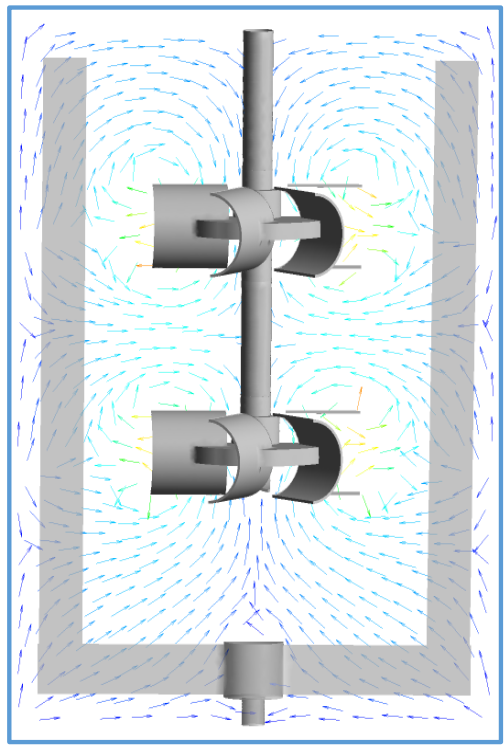

(f)

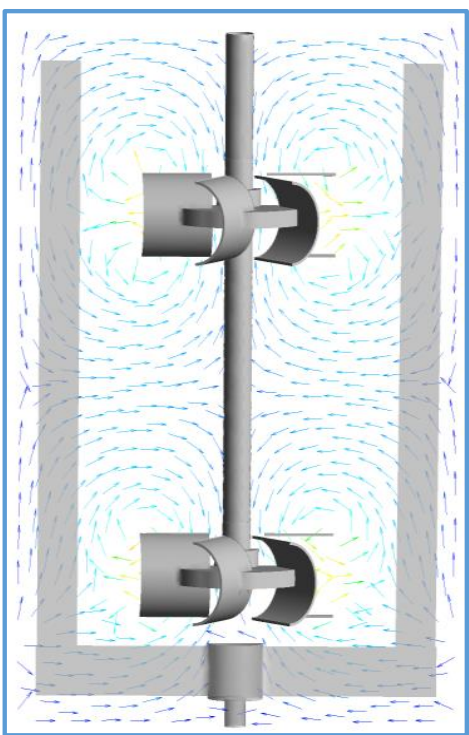

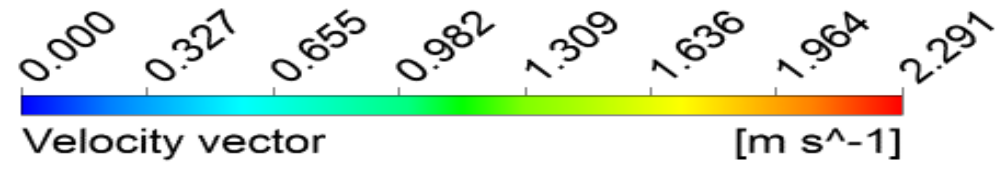

Figure (5.4-5) Velocity $(\mathrm{m} / \mathrm{s})$ vector plots generated by different coaxial mixing configurations at $R_{n}=8\left(N_{a}=30 \mathrm{rpm}\right.$ and $\left.N_{s}=240 \mathrm{rpm}\right)$ and $1.5 \%$ xanthan gum solution in the co-rotating mode: (a) SS1, (b) SS2, (c) SS3, (d) SS4, (e) SS5, and (f) SS6. 
(a)

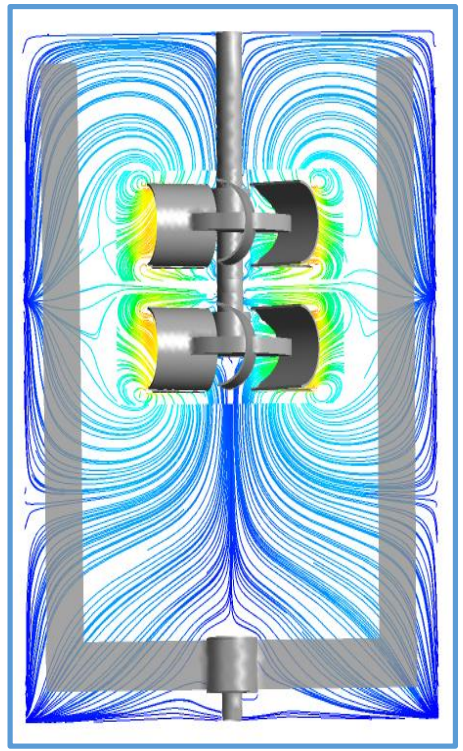

(d)

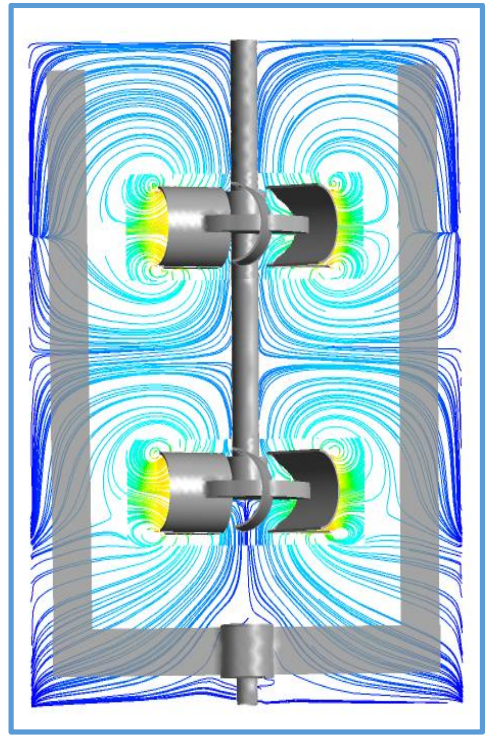

(b)

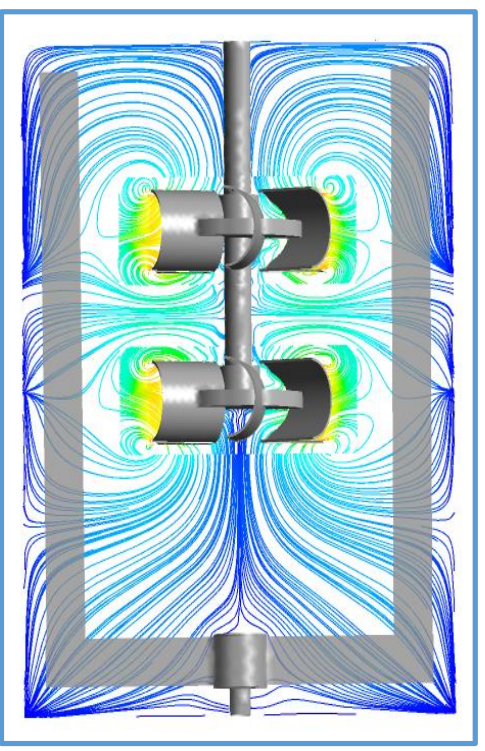

(e)
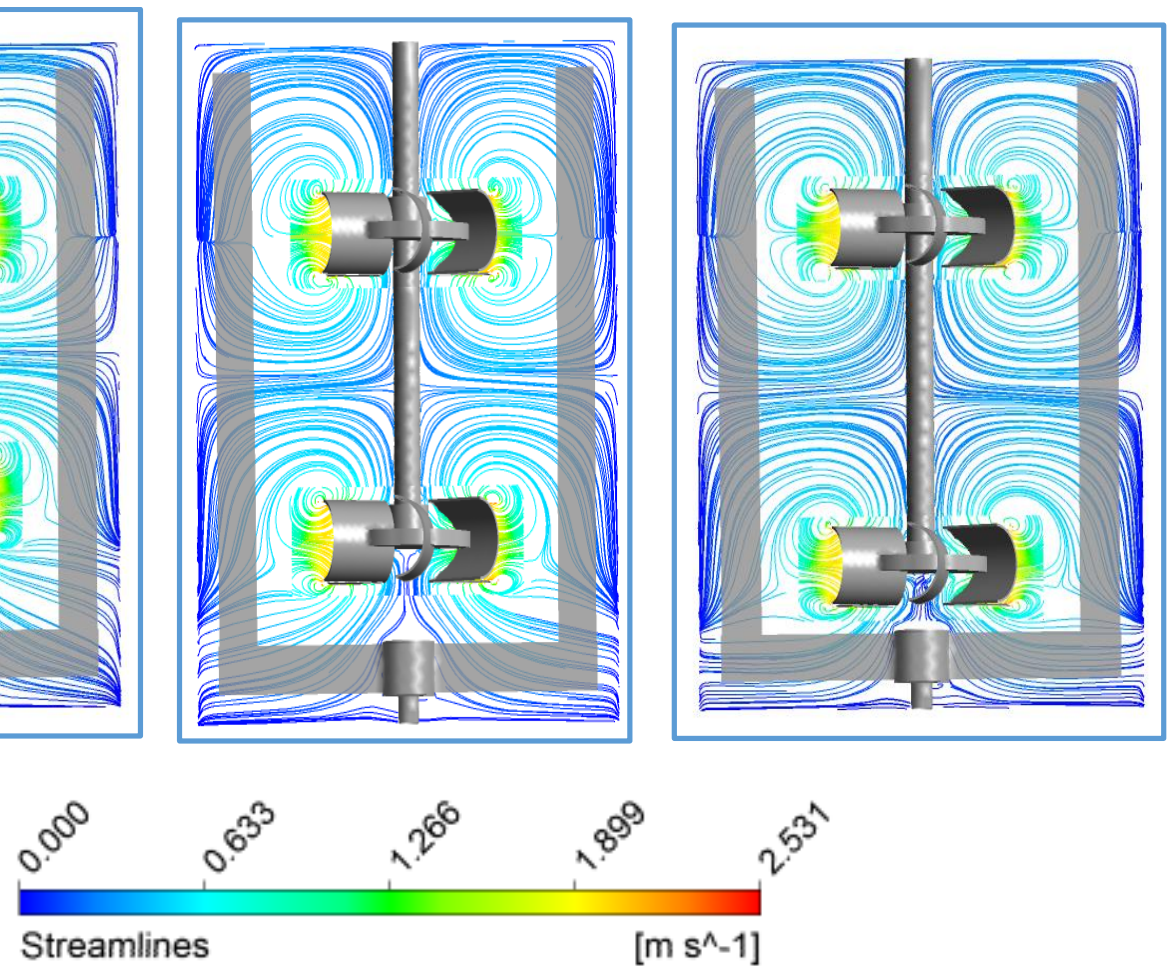

(c)

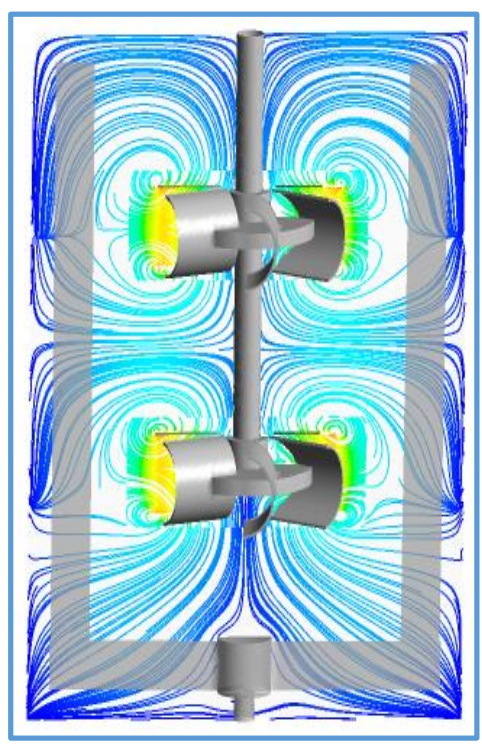

(f)

Figure (5.4-6). Streamlines $(\mathrm{m} / \mathrm{s})$ plots generated by different coaxial mixing configurations at $R_{n}=8\left(N_{a}=30 \mathrm{rpm}\right.$ and $\left.N_{s}=240 \mathrm{rpm}\right)$ and $1.5 \%$ xanthan gum solution in the co-rotating mode: (a) SS1, (b) SS2, (c) SS3, (d) SS4, (e) SS5, and (f) SS6. 
To quantitatively compare the performances of different coaxial mixing configurations in the co-rotating mode, the CFD model was used to obtain the dimensionless axial profiles of the radial, axial, and tangential velocities along the vessel height at a radial location close to the impeller tip, $2 r / T=0.55$, and these profiles are shown in Figure (5.4-7). Figure (5.4-7) a shows the radial velocity profiles inside the tank for different configurations of the coaxial mixer. The maximum radial velocities attained by the upper impeller $(Z / H=0.72)$ for the SS1, SS2, SS3, SS4, SS5, and SS6 configurations were $0.026 V_{t i p}, 0.034 V_{t i p}, 0,044 V_{t i p}, 0.043 V_{t i p}, 0.045 V_{t i p}$, and $0.050 V_{t i p}$, respectively. The maximum radial velocity achieved by the lower impellers were $0.026 V_{t i p}, 0.035 V_{t i p}$, $0,040 V_{t i p}, 0.042 V_{t i p}, 0.045 V_{t i p}$, and $0.053 V_{t i p}$, respectively. These results show that for the SS3, SS4, SS5, and SS6 configurations, the maximum radial velocities achieved by the upper impeller were higher than those generated by the corresponding lower impeller with an increased impeller spacing $\left(C_{2}\right)$. The comparison of the radial velocity profiles for different coaxial mixing configurations demonstrates that the highest radial velocities of $V_{r}=0.053 V_{t i p}$ and $V_{r}=0.045 V_{t i p}$ were generated by the lower and upper impellers, respectively, for the SS6 configuration. However, the magnitude of the radial velocities between two central Scaba impellers for the SS1, SS2, SS3, SS4, SS5, and SS6 configurations were $0.026 V_{t i p}, 0.069 V_{t i p}, 0,093 V_{t i p}, 0.093 V_{t i p}, 0.09 V_{t i p}$, and $0.087 V_{t i p}$. It can be seen that the highest radial velocities between two central impellers were achieved by the SS3 and SS4 configurations.

Figure (5.4-8) b illustrates the axial velocity profiles along the height of the tank at $2 r / T=$ 0.55. These data demonstrate that the SS2 configuration produced the strongest axial velocities from the upper part to the lower part of the tank compared to the other 
configurations with the maximum axial velocity of $V_{a}=0.088 V_{\text {tip }}$. In fact, due to a smaller impeller spacing for the SS2 configuration, this coaxial mixing configuration acted as a single impeller with a width greater than twice of the single Scaba impeller. In this configuration, the tangential and axial velocities were increased due to the positive effect of the anchor impeller. As a result, a larger pseudo-cavern was formed and the generation of this larger cavern intensified the upward and downward motion of the fluid inside of the tank (Farhat et al., 2007). However, for the SS2 coaxial mixer, the stagnant and/or slow-moving fluid in the region under the double central Scaba impellers were higher compared to the other configurations [Figure (5.4-4)]. This can be occurred due to the inadequate radial pumping in the lower part of the tank.

Figure (5.4-7) c depicts the profile of the dimensionless tangential velocity along the tank wall at $2 r / T=0.55$ for different coaxial mixing configurations. As it can be noted, the maximum dimensionless tangential velocity $\left(V_{t}=0.34 V_{t i p}\right)$ was generated by the SS1 configuration in the upper part of the tank. In fact, due to a small impeller spacing, the combination of the central impellers in this configuration acted as a single impeller and generated the highest tangential velocity. It must be mentioned that the magnitude of the tangential velocity generated by the central impellers are enhanced by the rotation of the anchor in the co-rotating mode (Farhat et al., 2007). Moreover, from Figure (5.4-7) c it can be seen that the SS2, SS3, SS4, SS5, and SS6 configurations generated almost the same maximum tangential velocity of $V_{t}=0.28 V_{t i p}$ (for the upper and the lower impellers) with a more uniform distribution of the tangential velocity from the upper part of the tank to the lower part of the tank . 
(a)

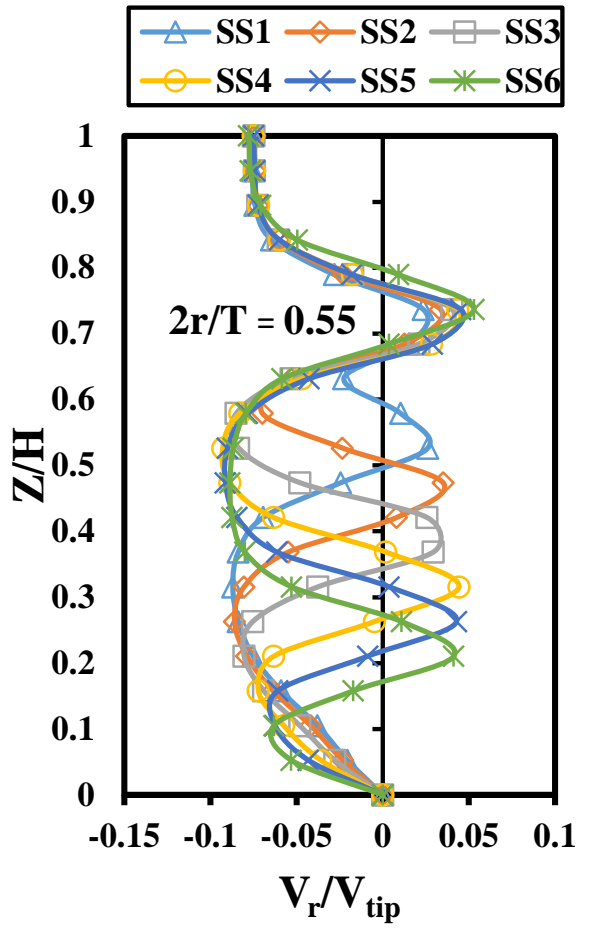

(b)

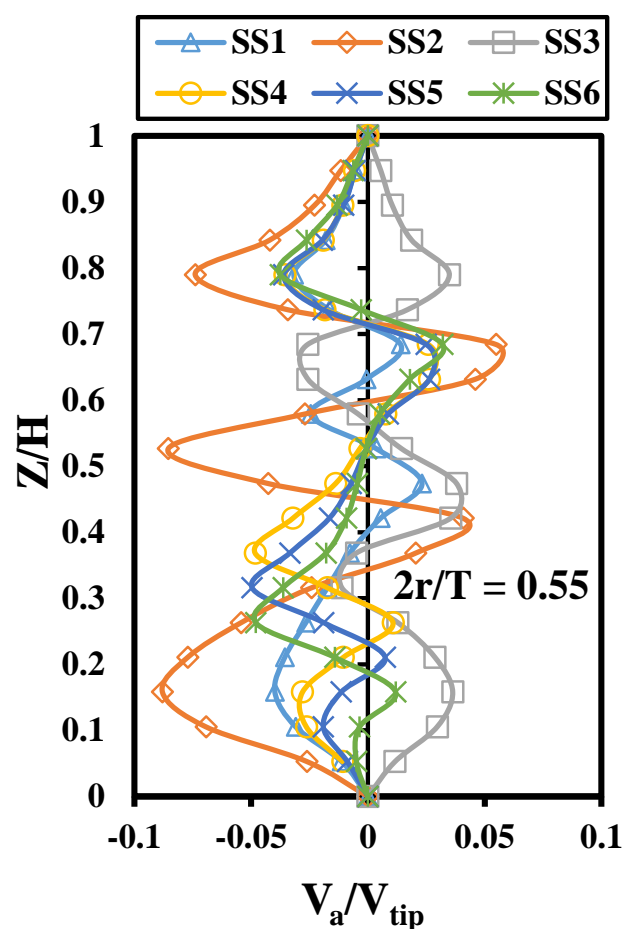

(c)

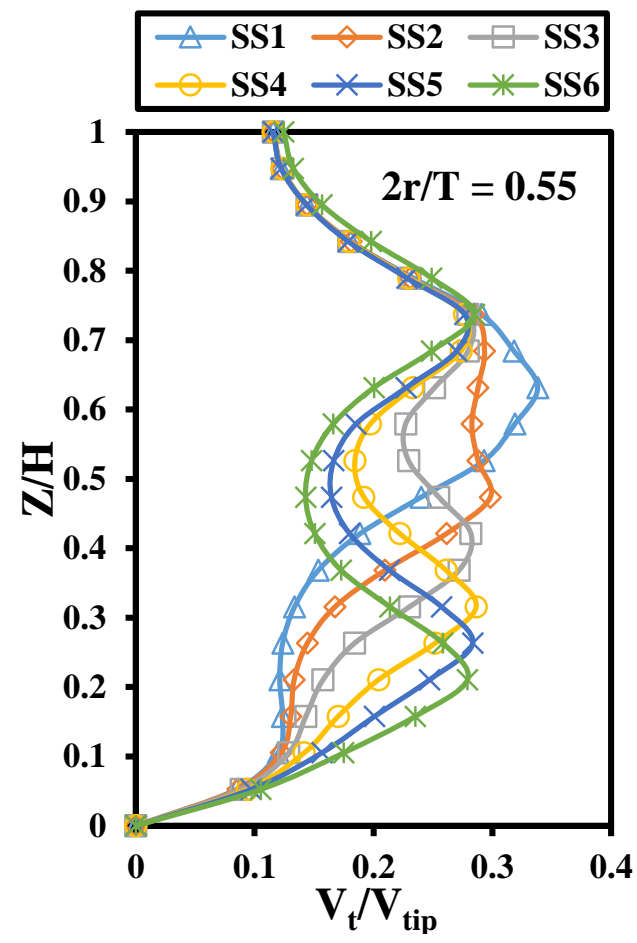

Figure (5.4-7). Axial profiles of the radial, axial, and tangential velocities at $2 r / T=0.55$ for different coaxial mixing configurations at $R_{n}=8\left(N_{a}=30 \mathrm{rpm}\right.$ and $\left.N_{s}=240 \mathrm{rpm}\right)$ and $1.5 \%$ xanthan gum solution in the co-rotating mode. 
Figure (5.4-8) depicts the radial profile of the radial velocities generated by the upper and the lower impellers at the centerlines of the upper and lower central impellers for the various coaxial mixing configurations. The maximum radial velocities for the upper impeller and the lower impeller were observed for the SS3, SS4, SS5, SS6 configurations at the tips of both impellers $(2 r / T=0.45)$. The maximum radial velocities created by the lower impellers of the SS3, SS4, SS5, and SS6 configurations were $0.115 V_{t i p}, 0.120 \mathrm{~V}_{\text {tip }}$, and $0.122 V_{t i p}$, and $0.121 V_{t i p}$, respectively. The maximum radial velocities for the upper impeller were $0.117 V_{t i p}, 0.123 \mathrm{~V}_{\text {tip }}, 0.125 V_{t i p}$, and $0.127 V_{t i p}$, respectively. The interaction between the flow streams generated by two central impellers were reduced with increasing the impeller spacing $\left(C_{2}\right)$. In fact, the parallel flow pattern was dominant at the higher impeller spacing. Such a behavior has been reported by Mishra and Joshi (1994) in the agitation of Newtonian fluids by double Rushton turbine impellers without anchor in a turbulent regime.

(a)

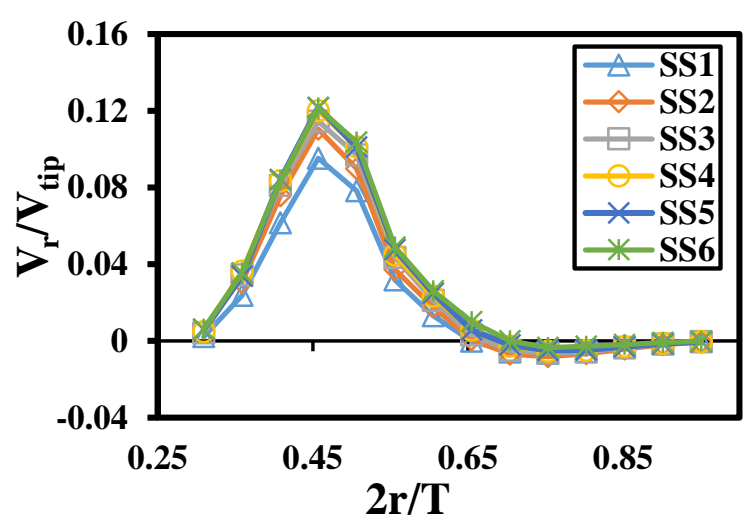

(b)

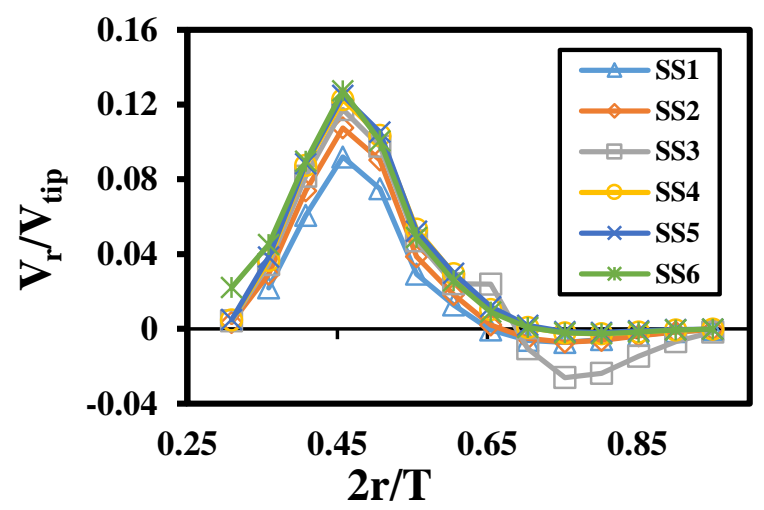

Figure (5.4-8). Radial profiles of the radial velocity at the impeller tip $\left(R_{\mathrm{i}}\right)$ for different coaxial mixing configurations at $R_{n}=8\left(N_{a}=30 \mathrm{rpm}\right.$ and $\left.N_{s}=240 \mathrm{rpm}\right)$ and $1.5 \%$ xanthan gum solution in the co-rotating mode: (a) lower impeller, and (b) upper impeller. 
To analyze the flow behavior in the regions above and below of the central impellers, the radial profiles of the axial and radial velocities at the axial levels of $0.015 \mathrm{~m}$ above and below each central impeller are shown in Figures (5.4-9), and (5.4-10), respectively. As it can be seen in Figure (5.4-9), the maximum axial velocities were observed by the SS3, SS4, and SS5 configurations with the impeller spacing $\left(C_{2}\right)$ in the range of $0.090 \mathrm{~m}$ $(0.18 H)$ to $0.252 \mathrm{~m}(0.50 H)$. This figure also shows that the best axial velocity profiles were achieved by the SS3 configuration above and below the upper impeller and below the lower impeller. However, the SS5 coaxial mixing configuration generated the maximum axial velocity above the lower impeller. Furthermore, Figures (5.4-10)a, and (5.4-10) b represent the radial profiles of the radial velocities at the axial level equal to $0.015 \mathrm{~m}$ above and below the upper impeller, respectively. It was found that the maximum radial velocity $\left(V_{r}=0.11 V_{t i p}\right)$ was induced by the SS3 configuration. However, as shown in Figures $(5.4-10) \mathrm{c}$, the maximum radial velocity $\left(V_{r}=0.092 V_{\text {tip }}\right)$ at $0.015 \mathrm{~m}$ above the lower impeller was observed for the SS1, SS2 and SS3 configurations. The reason is that the interaction between two returning flow streams from the upper and lower impellers was increased by decreasing the impeller spacing $\left(C_{2}\right)$ and thus intensified the radial flow as observed in Figure (5.4-6) as well. Moreover, the lowest radial velocities at $0.015 \mathrm{~m}$ below the lower impeller were attained by the SS5 and SS6 coaxial mixer configurations. In fact, for the impeller spacing $\left(C_{2}\right)$ greater than $0.200 \mathrm{~m}$, the radial velocity below the lower central impeller decreased due to the interaction between the flow created by the anchor impeller and the lower impeller. Also, this interaction may require higher power drawn as shown in Table (5.4-4). 
(a)

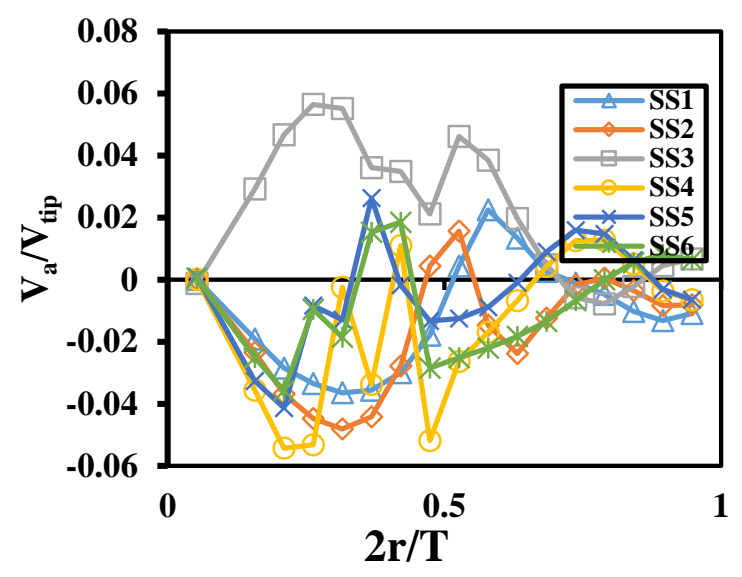

(c)

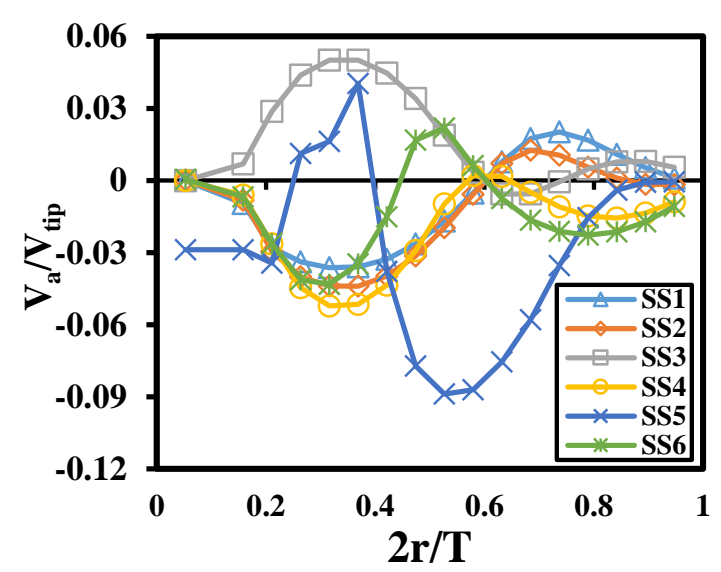

(b)

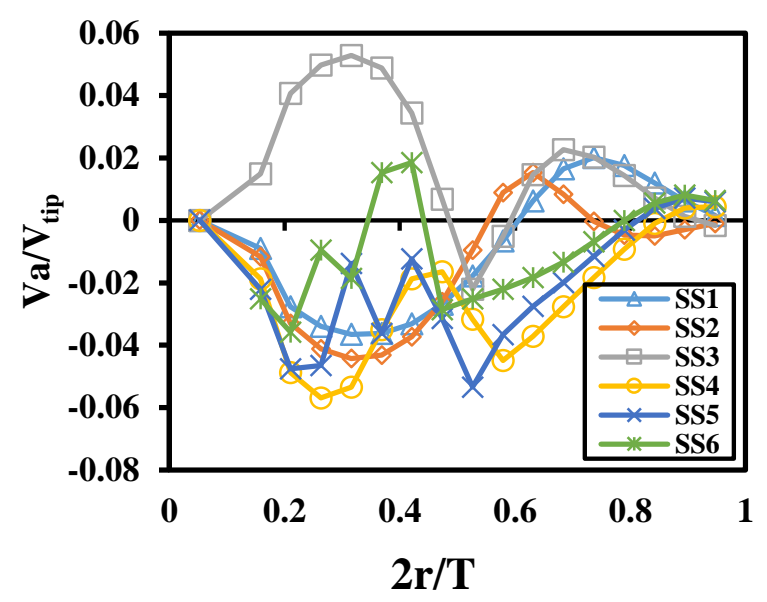

(d)

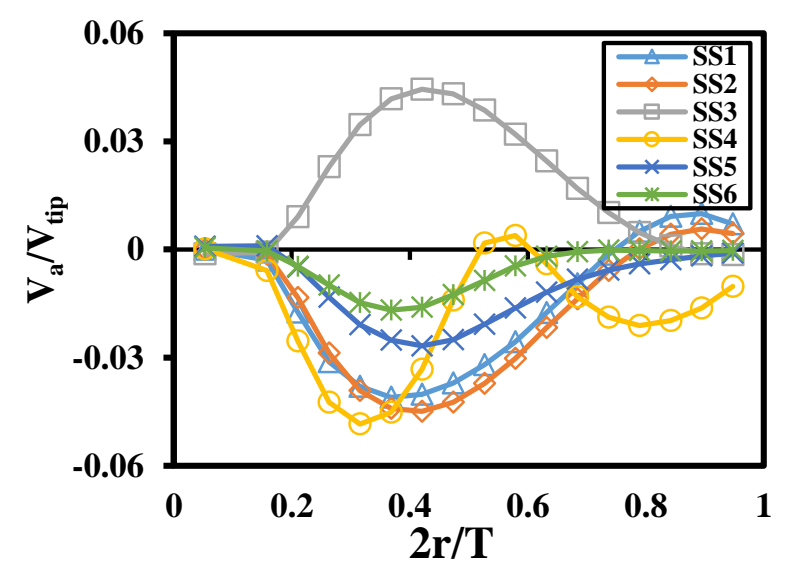

Figure (5.4-9). Radial profiles of the axial velocity at $R_{n}=8\left(N_{a}=30 \mathrm{rpm}\right.$ and $N_{s}=240$ $\mathrm{rpm}$ ) and $1.5 \%$ xanthan gum solution in the co-rotating mode for: (a) $15 \mathrm{~mm}$ above the upper impeller, (b) $15 \mathrm{~mm}$ below the upper impeller, (c) $15 \mathrm{~mm}$ above the lower impeller, and (d) $15 \mathrm{~mm}$ below the lower impeller. 
(a)

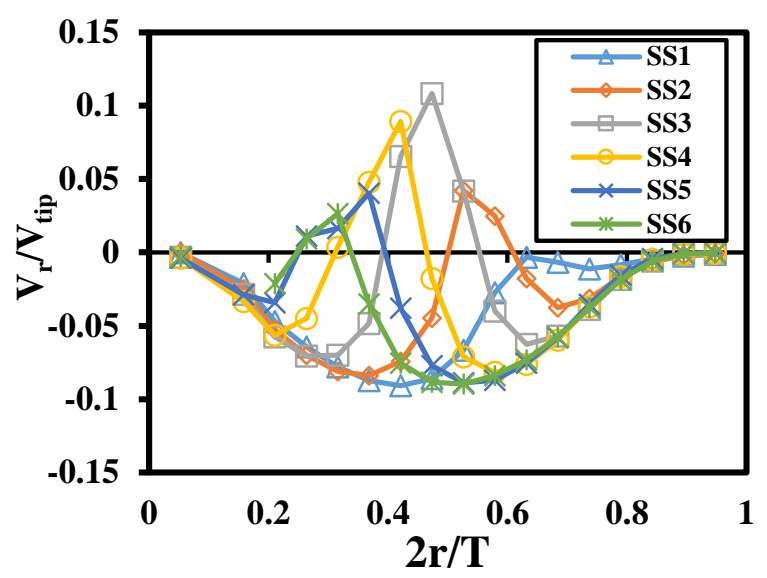

(c)

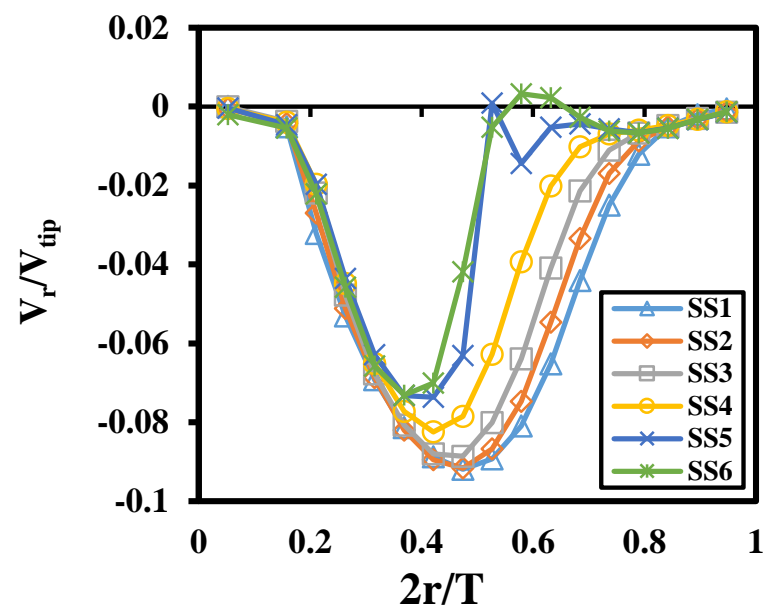

(b)

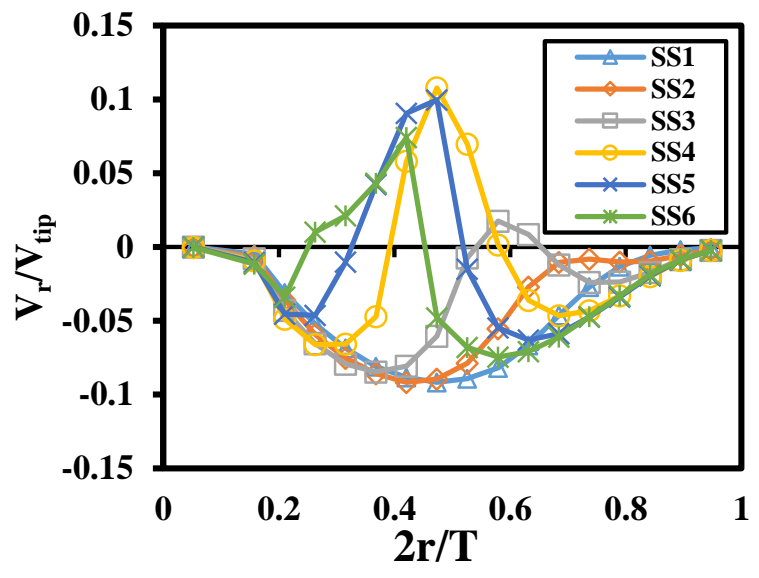

(d)

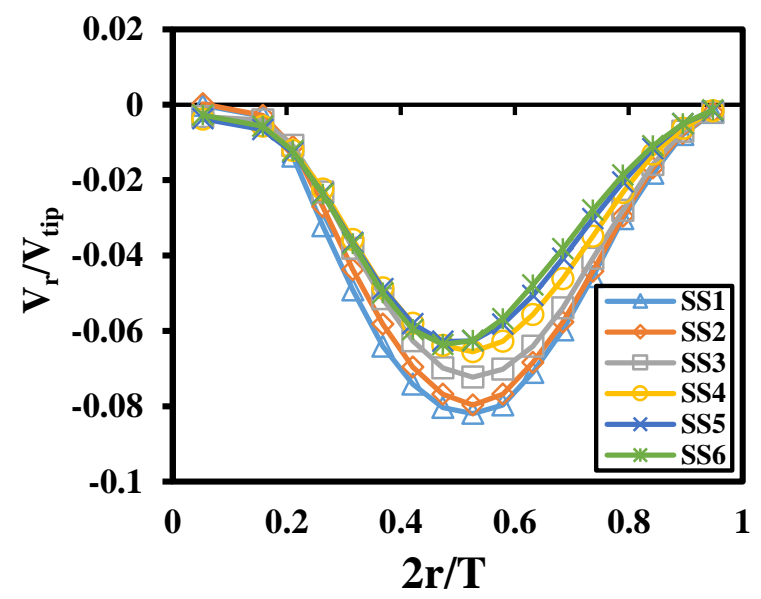

Figure (5.4-10). Radial profile of the radial velocity at $R_{n}=8\left(N_{a}=30 \mathrm{rpm}\right.$ and $N_{s}=240$ $\mathrm{rpm}$ ) and $1.5 \%$ xanthan gum solution in the co-rotating mode for: (a) $15 \mathrm{~mm}$ above the upper impeller, (b) $15 \mathrm{~mm}$ below the upper impeller, (c) $15 \mathrm{~mm}$ above the lower impeller, and (d) $15 \mathrm{~mm}$ below the lower impeller.

The apparent viscosity of a non-Newtonian highly viscus fluid depends on the shear rate.

Thus, for a better flow field analysis generated by the coaxial mixers utilized in this 
study, the dimensionless average shear strain rates $\left(\gamma_{\text {ave }}^{\cdot} / N_{c o}\right)$ along the tank height are presented in Figure (5.4-11). This figure shows that the highest average shear strain rates $\left(\gamma_{\text {ave }}^{\cdot} / N_{c o}\right)$ induced by the upper impeller $(Z / H=0.72)$ at $2 r / T=0.55$ were $3.46 N_{c o}, 3.46$ $N_{c o}, 3.20 N_{c o}, 3.20 N_{c o}, 3.10 N_{c o}$, and $3.30 N_{c o}$ for the SS1, SS2, SS3, SS4, SS5, and SS6 coaxial mixers, respectively. However, the average shear strain rates achieved by the lower impeller for the SS1, SS2, SS3, SS4, SS5, and SS6 configurations were $3.46 N_{c o}$, $3.37 N_{c o}, 3.19 N_{c o}, 3.20 N_{c o}, 3.10 N_{c o}$, and $3.03 N_{c o}$, respectively. These data demonstrate that the highest average shear strain at the upper part of the vessel $(Z / H=0.50)$ was achieved by the SS1 and SS2 coaxial mixers while the shear strain rates induced by these two coaxial mixers were decreased from $Z / H=0.00$ to $Z / H=0.50$. In these two configurations, both impellers acted like a single impeller with a larger blade width. This generated adequate motion in the upper part of the tank with the extensive dead or near stagnant zones in the rest of the tank particularly between the lower central impeller and the anchor impeller [Figure (5.4-4)]. Furthermore, among the SS3, SS4, SS5 and SS6 configurations, the SS3 and SS4 coaxial mixers generated the highest average shear strain rates of $3.26 N_{c o}$ and $3.20 N_{c o}$ by the upper and the lower impellers, respectively. It can also be noticed that the maximum average shear strain rate of $\gamma_{\text {ave }}^{\cdot}=2.42 N_{c o}$ between two central impellers was generated by the SS3 configuration. It must be mentioned that the higher shear strain rate between two central impellers resulted in the lower compartmentalization between the impellers. Therefore, it can be concluded that a better deformation profiles was generated by the SS3 configuration compared to the other configurations. 


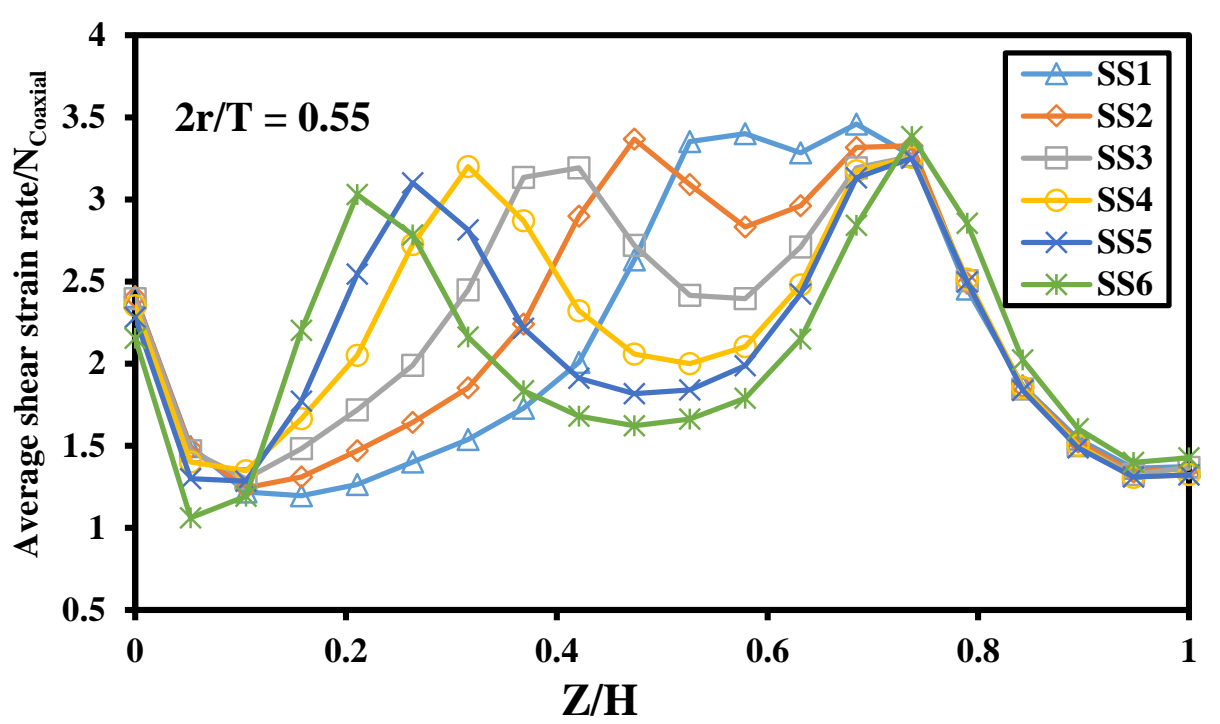

Figure (5.4-11). Dimensionless average shear strain rate plots along the tank wall generated by different coaxial mixing configurations at $R_{n}=8\left(N_{a}=30 \mathrm{rpm}\right.$ and $N_{s}=240$ $\mathrm{rpm}$ ) and 1.5\% xanthan gum solution in the co-rotating mode: (a) SS1, (b) SS2, (c) SS3, (d) SS4, (e) SS5, and (f) SS6.

Figure (5.4-12) illustrates the apparent viscosity contour plots for various configurations of the coaxial mixers employed in this study. Figure (5.4-12) a shows that both central impellers operated as a single impeller due to a small impeller spacing resulting in the formation of a single cavern in the upper part of the tank. In fact, a low-viscosity region (a higher-shear rate zone) formed around the central impellers while the high-viscosity zones were generated in the upper part and the lower part of the tank even between two impellers. Moreover, the results presented in Figures (5.4-12) b to (5.4-12) e show that the high-viscosity regions were decreased significantly by increasing the distance between two central impellers $\left(C_{2}\right)$. However, it must be mentioned that this reduction was much higher for the SS3 and SS4 compared to those for the SS5 and SS6 coaxial mixing configurations. 
(a)

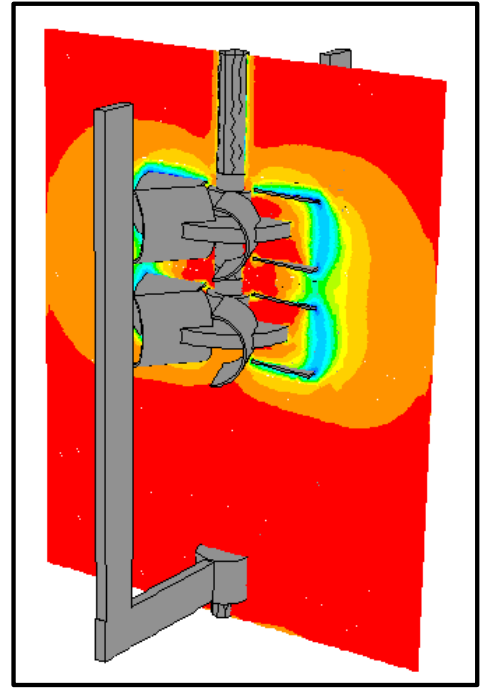

(d)

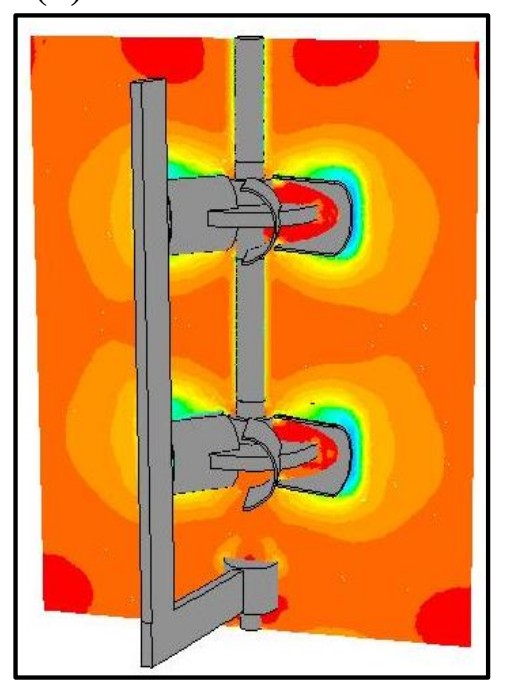

(b)

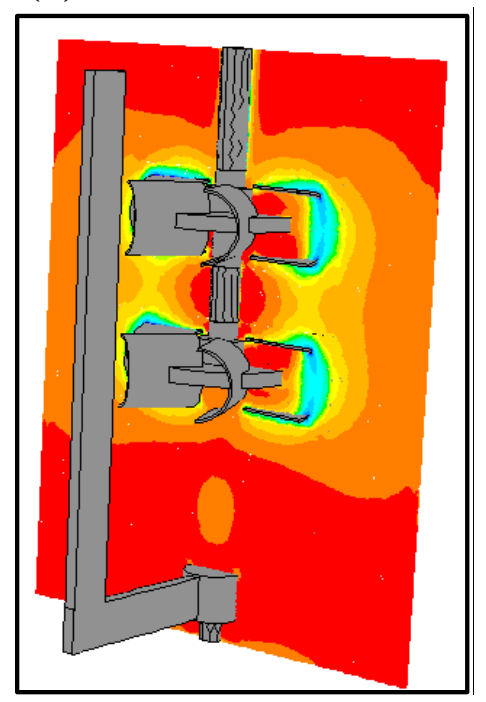

(e)

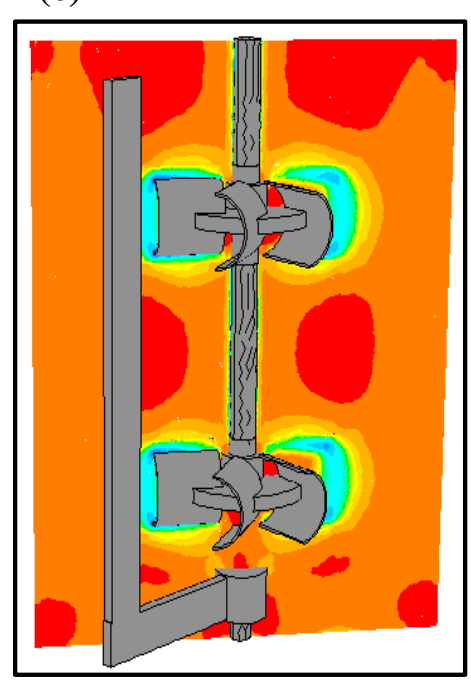

(c)

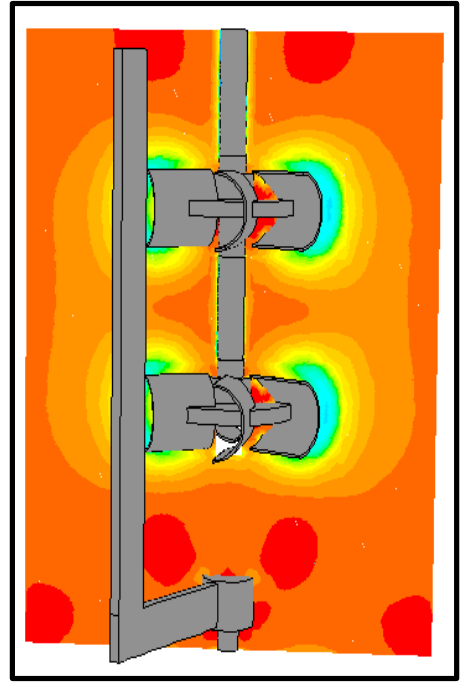

(f)

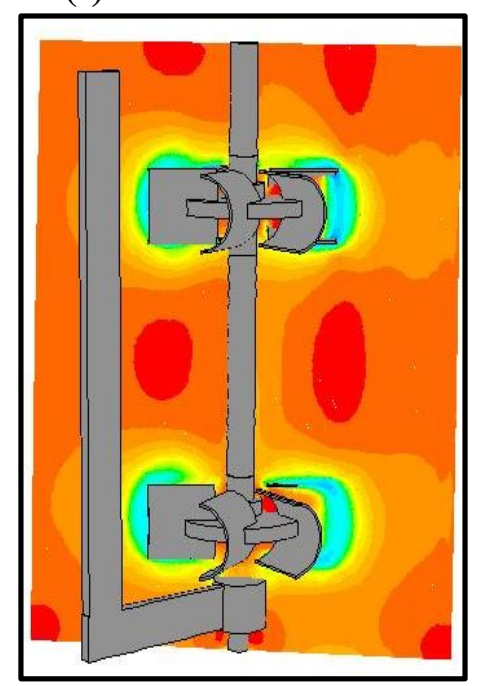

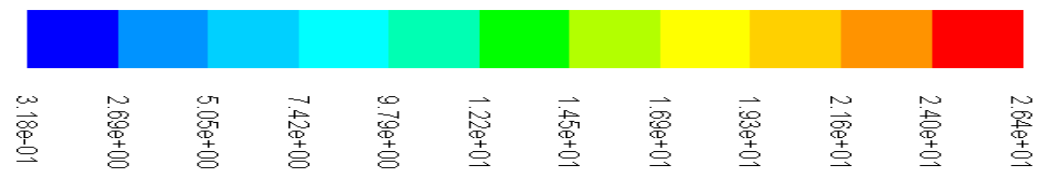

Figure (5.4-12). Viscosity (Kg/m-s) contour plots generated by different coaxial mixing configurations at $R_{n}=8\left(N_{a}=30 \mathrm{rpm}\right.$ and $\left.N_{s}=240 \mathrm{rpm}\right)$ and $1.5 \%$ xanthan gum solution in the co-rotating mode: (a) SS1, (b) SS2, (c) SS3, (d) SS4, (e) SS5, and (f) SS6. 


\subsubsection{Conclusion}

The objective of this section was to investigate the influence of the impeller spacing on the performance of a coaxial mixer composed of two central Scaba impellers and an anchor in the agitation of highly viscous non-Newtonian fluids in co-rotating mode. To fulfill this objective, the advanced computational fluid dynamics (CFD) and the electrical resistance tomography (ERT) were adopted. The CFD model was validated by comparing the experimentally determined power drawn and mixing time data with the computed values attained by the CFD model for different configurations of the coaxial mixer. Power number, flow number, mixing time, and pumping effectiveness were employed to assess the performances of the coaxial mixer as a function of the spacing between two central impellers for both co-rotating and counter-rotating modes. It was found out that the mixing in co-rotating mode was more efficient than the counter-rotating mode. In order to obtain the optimal impeller spacing, the energy consumption, which is the product of the power consumption and mixing time, was calculated for various impeller spacing. It was found that the coaxial mixer with the impeller spacing of $0.175 \mathrm{~m}$ and the impeller clearance of $0.185 \mathrm{~m}$ was the most efficient system with the minimum energy consumption among the investigated configurations. The velocity vector plot, velocity contour plot, streamlines plot, axial velocity profile, radial velocity profile, tangential velocity profile, shear rate profile, and apparent viscosity profile were calculated through the validated CFD model to analyze the characteristic flow pattern generated by the double Scaba-anchor coaxial mixer as a function of the spacing between two central Scaba impellers. These data indicated that the changes in the impeller spacing resulted in two stable flow patterns: parallel flow and merging flow. 


\subsection{Mixing of non-Newtonian biopolymer solutions with the coaxial mixers composed of two different central impellers and an anchor}

\subsubsection{Introduction}

The objective of this part of our study was to explore and compare the hydrodynamic performances of different configurations of coaxial mixers composed of a wall scraping anchor impeller in combination with two different or identical central high speed impellers in the agitation of the yield-pseudoplastic xanthan gum solution. The coaxial mixers utilized in this study were the Scaba-Scaba-anchor (SSAC), Scaba-Rushtonanchor (SRAC), Rushton-Scaba-anchor (RSAC), Scaba-pitched blade-anchor (SPAC), and pitched blade-Scaba-anchor (PSAC)[Figure (5.5-1)]. The performances of these coaxial mixers were investigated both numerically and experimentally in the laminartransitional regime. The computational fluid dynamics (CFD) and electrical resistance tomography (ERT) were employed for the 3D numerical simulations of the flow domain and mixing time measurements, respectively. A new correlation was introduced for these complex configurations of the coaxial mixers by incorporating the Metzner-Otto constants $\left(K_{s}\right)$ of the different types of the central impellers into the Reynolds number. Furthermore, the performances of the coaxial mixers were assessed with respect to the power consumption, mixing time, fluid velocity profiles, flow number, pumping effectiveness, and mixing efficiency. 

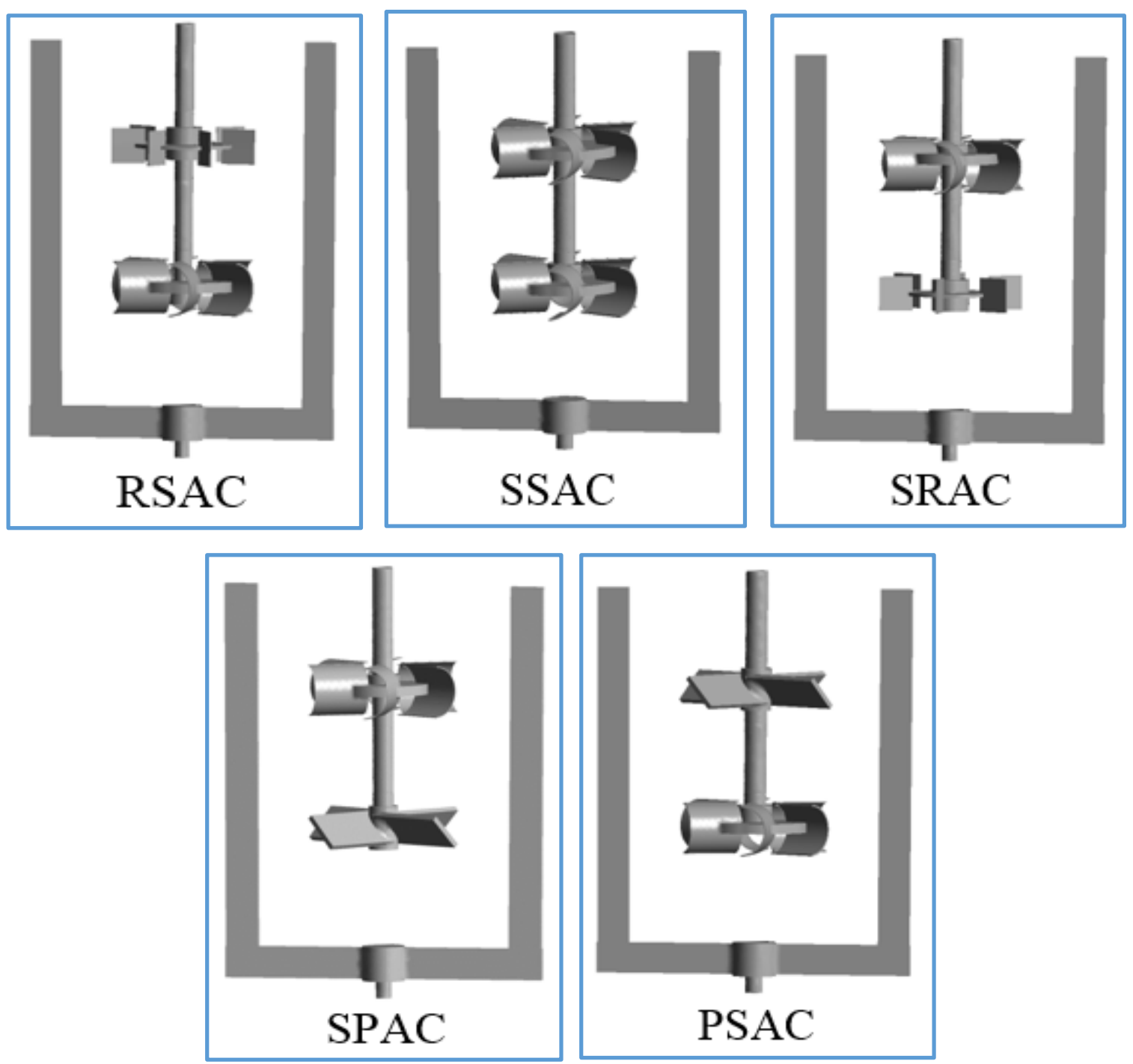

Figure (5.5-1). Different configurations of coaxial in this study.

The CFD results for the specific power consumption and mixing time were compared to the experimentally obtained data to validate the CFD model. The results listed in Table (5.5-1) show a good agreement between the computed and experimentally measured values with a maximum standard deviation of less than $5 \%$. The validated model was then employed to produce further information in this study. 
Table (5.5-1). Computed and measured power consumption and mixing time for five coaxial mixers at $R_{n}=8\left(N_{a}=30 \mathrm{rpm}\right.$ and $\left.N_{s}=240 \mathrm{rpm}\right), 1.5 \%$ xanthan gum solution, and co-rotating mode.

\begin{tabular}{c|ccc|ccc}
\hline $\begin{array}{c}\text { Coaxial } \\
\text { mixer }\end{array}$ & $\begin{array}{c}P_{t o t} / V \\
\text { Exp. }\left(\mathrm{W} / \mathrm{m}^{3}\right)\end{array}$ & $\begin{array}{c}P_{t o t} / V \\
\text { Num. }\left(\mathrm{W} / \mathrm{m}^{3}\right)\end{array}$ & $\begin{array}{c}\text { error } \\
(\%)\end{array}$ & $\begin{array}{c}\text { Mixing } \\
\text { time } \\
\text { Exp. }(\mathrm{min})\end{array}$ & $\begin{array}{c}\text { Mixing } \\
\text { time } \\
\text { Num. } \\
(\mathrm{min})\end{array}$ & $\begin{array}{c}\text { error } \\
(\%)\end{array}$ \\
\hline SSAC & 1379.10 & 1397.19 & 1.30 & 7.38 & 7.75 & 4.70 \\
SRAC & 1282.40 & 1301.03 & 1.40 & 9.75 & 10.25 & 4.90 \\
RSAC & 1312.30 & 1350.97 & 2.90 & 10.59 & 10.93 & 3.10 \\
SPAC & 1103.20 & 1145.96 & 3.70 & 7.20 & 7.55 & 4.6 \\
PSAC & 1016.60 & 1068.84 & 4.90 & 9.34 & 9.82 & 4.90 \\
\hline
\end{tabular}

\subsubsection{Results and Discussion}

\subsubsection{Power Consumption}

Pakzad et al. (2013b) proposed the following correlations for the power number and Reynolds number of the coaxial mixers composed of an anchor and a single central impeller in the agitation of the yield-pseudoplastic fluids:

$$
\begin{gathered}
N_{p(\text { coaxial })}=\frac{P_{\text {tot }}}{\rho\left(N_{c}+f_{p(a)} N_{a}\right)^{3} D_{c}^{5}} \\
\operatorname{Re}=\frac{K_{S(c)}\left(N_{c}+f_{p(a)} N_{a}\right)^{2} D_{c}{ }^{2} \rho}{\left[\tau_{y}+K\left(K_{S(c)}\right)^{n}\left(N_{c}+f_{p(a)} N_{a}\right)^{n}\right]}
\end{gathered}
$$

As can be seen in Equation (5.5-2), in order to calculate the Reynolds number in the agitation of non-Newtonian yield-pseudoplastic fluids, they incorporated the $K_{s}$ value of 
the central impeller into the Reynolds number by using the Metzner and Otto approach (1957):

$$
\dot{\gamma}=K_{s} N
$$

Kazemzadeh et al. (2016b) showed that Equation (5.5-2) can also be used for the coaxial mixers comprised of an anchor impeller and two identical central impellers.

In this study, it was required to determine the $K_{s}$ value for the coaxial mixers composed of two different types of the central impellers (i.e. Scaba-Rushton and Scaba-pitched blade). In order to achieve this goal, at the first stage, the average shear rates of the individual impellers (Scaba, Rushton, and pitched blade) were calculated by the CFD model for the volume of the fluid surrounding each individual impeller with a height of $0.08 \mathrm{~m}$ and a diameter of $0.220 \mathrm{~m}$ (Pakzad et al., 2008a). Figure (5.5-2) depicts the average shear rate versus the impeller speed at three different xanthan gum concentrations for the Scaba, Rushton, and pitched blade impellers. These data show a linear relation between the average shear rate and the rotational speed of the impeller. By applying the regression analysis, the computed $K_{S}$ values for the Scaba, Rushton, and pitched blade impellers were $9.234,8.320$, and 8.021 , respectively. These values were in good agreement with $K_{s}=9.560$ for the Scaba reported by Torrez and Andre (1998), $K_{s}=$ 8.50 for the Rushton determined by Torrez and Andre (1999), and $K_{s}=8.580$ for the pitched blade turbine measured by Rudolph et al. (2007) with a relative error of 3.4\%, $2.1 \%$, and $6.5 \%$, respectively. Moreover, the results presented in Figure (5.5-2) for three different xanthan gum concentrations show that the $K_{s}$ value was not dependent on the rheology of the fluid as expected. 
(a)

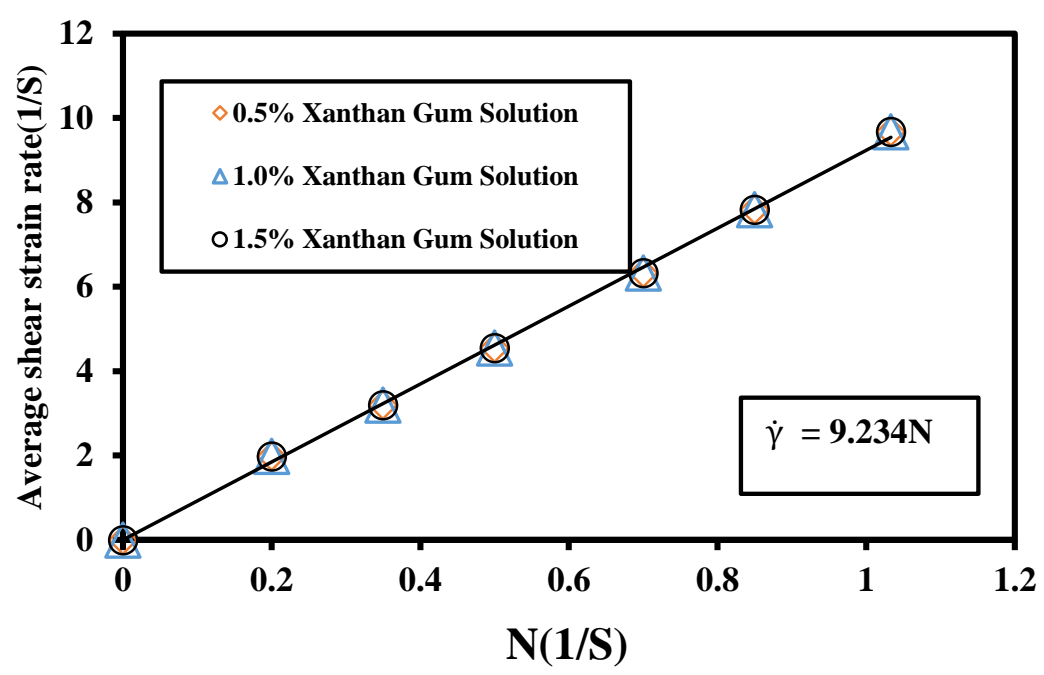

(b)

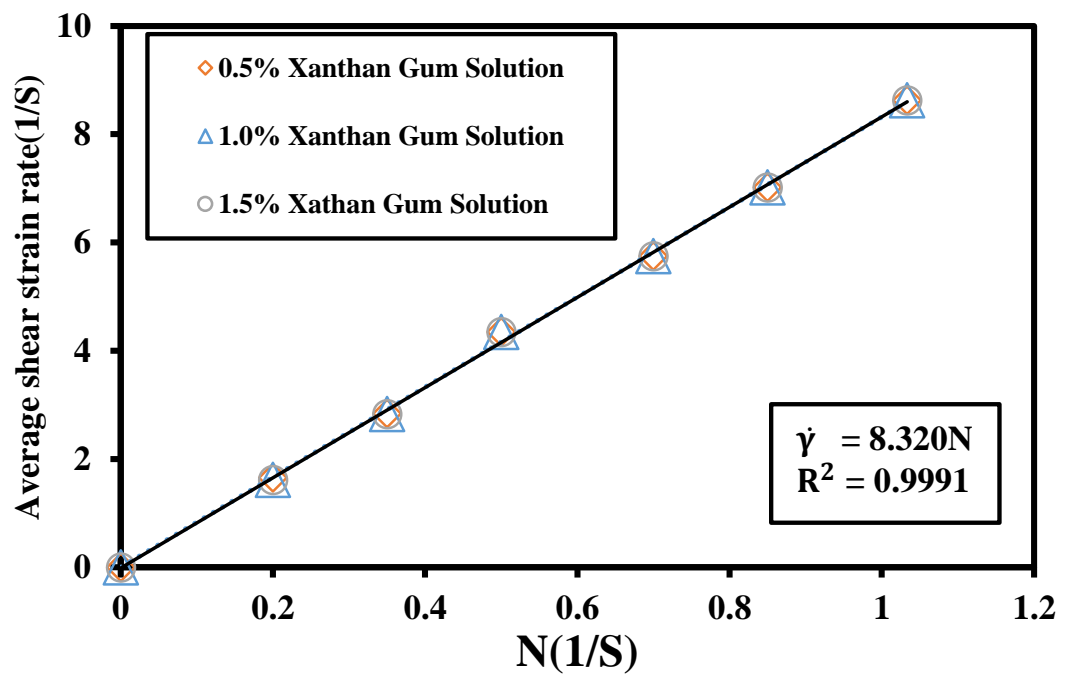


(c)

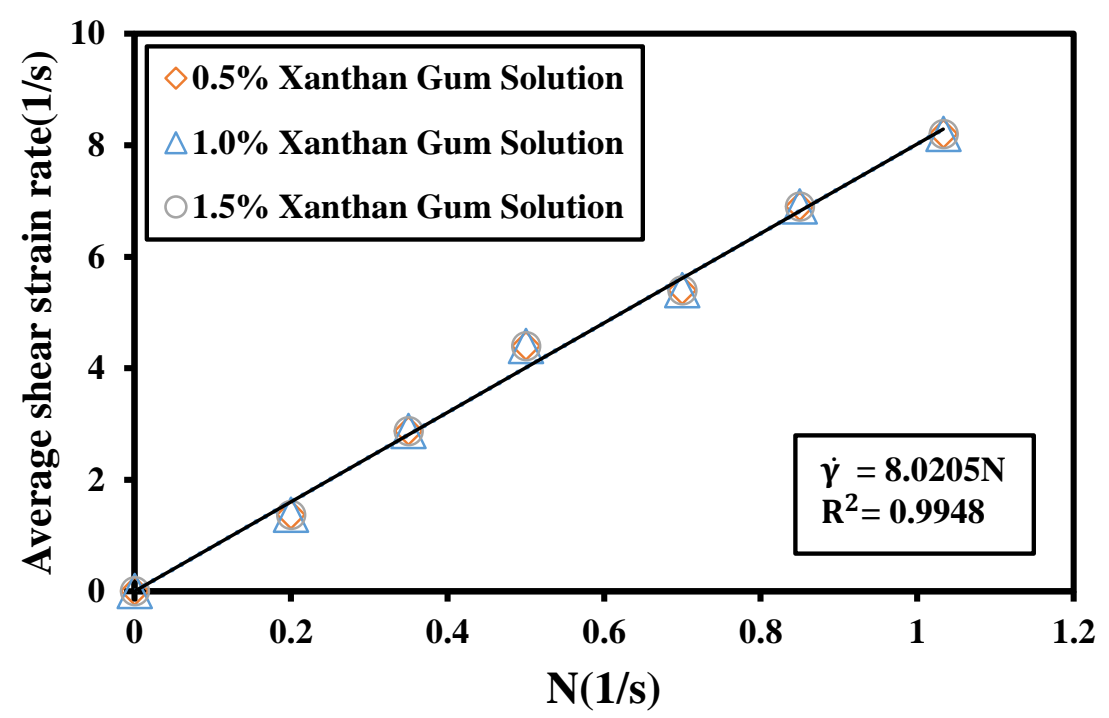

Figure (5.5-2). Average shear rate (1/s) versus the impeller speed (1/s) at $R_{n}=8$ and corotating mode as a function of the xanthan gum concentration for: (a) single Scaba, (b) single Rushton, and (c) single pitched blade impellers.

The same approach was applied to calculate the average shear rates for the double central impellers (i.e. the Scaba- pitched blade and the Scaba-Rushton). Figure (5.5-3) illustrates a linear relation between the average shear rate and the rotational speed of the double central impellers at three different xanthan concentrations. Furthermore, by applying the regression analysis, the $K_{s}$ values of 9.210 and 8.700 were obtained for the ScabaRushton and Scaba-pitched blade, respectively. 
(a)

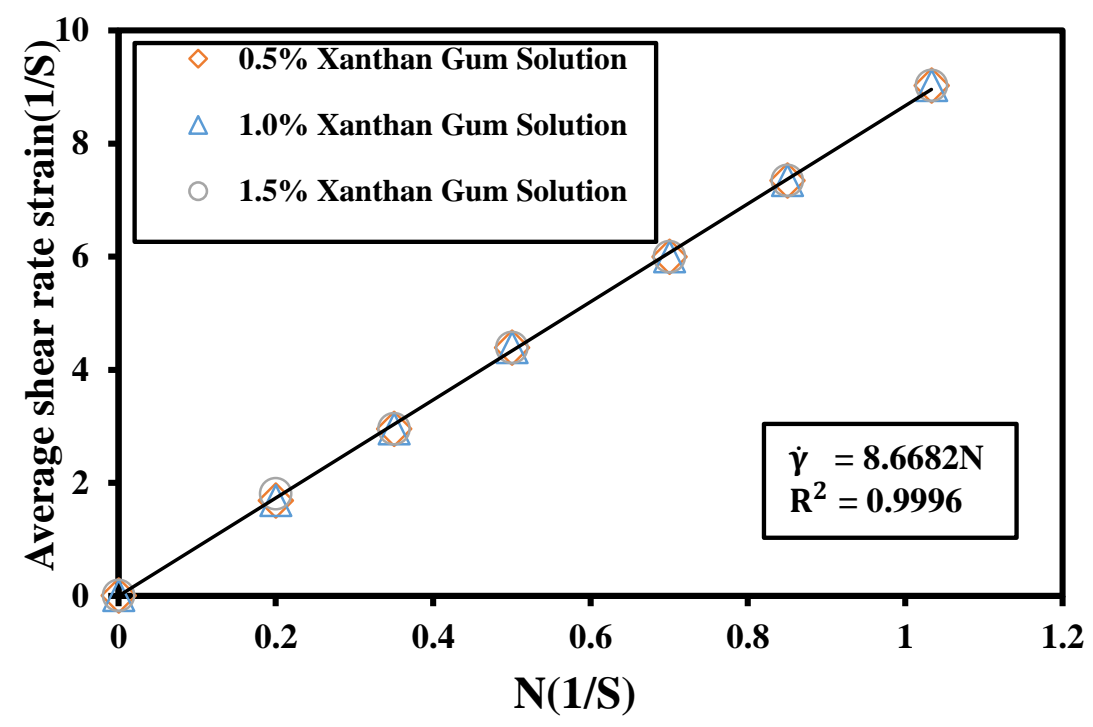

(b)

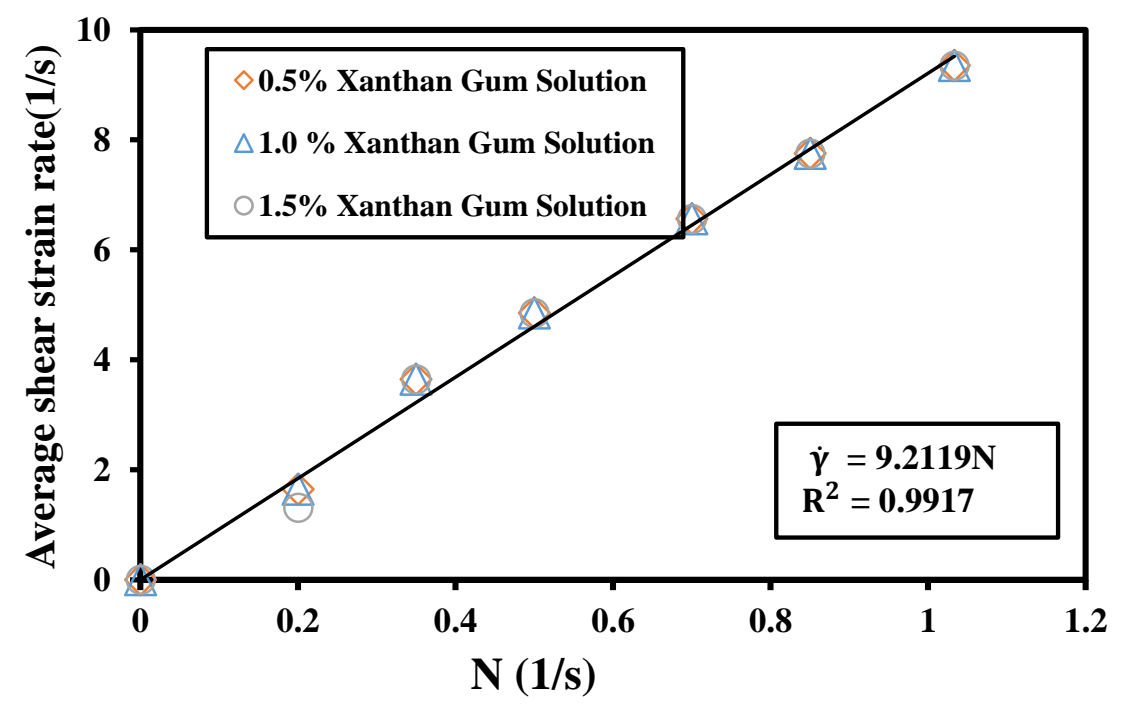

Figure (5.4-3). Average shear rate (1/s) versus the impeller speed (1/s) at $R_{n}=8$ and corotating mode as a function of the xanthan gum concentration for: (a) Scaba-pitched blade coaxial mixer, and (b) Scaba-Rushton-anchor coaxial mixer. 
The analysis of the calculated $K_{s}$ values for the single and double impellers proved that the $K_{s}$ values of the Scaba-Rushton and Scaba-pitched blade were almost equal to the average values of $K_{s}$ obtained for each individual impeller:

$$
K_{s c}=\frac{K_{s l i}+K_{s u i}}{2}
$$

where $K_{s c}$ is the Metzner and Otto constant for the double central impeller, $K_{s l i}$ is the Metzner-Otto constant for the lower impeller, and $K_{\text {sui }}$ is the Metzner-Otto constant for the upper impeller. For instance, the average of $K_{s}$ values obtained for the Scaba and the pitched blade impellers was 8.628 while the computed $K_{s}$ value for the Scaba-pitched blade was 8.700 with a relative error of less than $1 \%$. The use of this approach resulted in a relative error of $4.7 \%$ for the Scabe-Rushton central impellers.

According to the abovementioned discussion, the Reynolds number proposed by Pakzad et al. (2013b) was modified for the coaxial mixing systems composed of an anchor and two different central impellers as follows:

$$
R e=\frac{\left(\frac{K_{s l i}+K_{s u i}}{2}\right)\left(N_{S c}+f_{p(a)} N_{a}\right)^{2} D_{s c}{ }^{2} \rho}{\left[\tau_{y}+K\left(\frac{K_{s l i}+K_{s u i}}{2}\right)^{n}\left(N_{c}+f_{p(a)} N_{a}\right)^{n}\right]}
$$

where $R e$ is Reynolds number; $K_{s l i}$ and $\mathrm{K}_{s u i}$ are the Metzner-Otto constants for the lower and the upper impeller; $\rho$ is the fluid density; $N_{c}$ is the rotational speed of the 
central double impeller; $N_{a}$ is the rotational speed of the anchor; $D_{c}$ is the central impeller diameter; and $f_{p(a)}$ is the anchor power fraction.

The master power curves ( $N_{p}$ vs $R e$ ) of five coaxial mixers investigated in this study for the laminar-transitional regime and the co-rotating mode at $N_{c}=50-250 \mathrm{rpm}$ and $N_{a}=5$ $25 \mathrm{rpm}$ and $1.5 \%$ xanthan gum solution are presented in Figure (5.5-4). This figure compares the power numbers of the investigated mixers as a function of the Reynolds number at the same conditions. It can be clearly noticed that the power curve of all coaxial mixers were overlapped at the lower Reynold numbers (i.e. $\mathrm{Re}<30$ ). However, the power consumptions of the SSAC and SRAC were higher compared to the others at the higher Reynolds numbers. This figure also demonstrates that the power consumption of the PSAC was less than the other coaxial mixers.

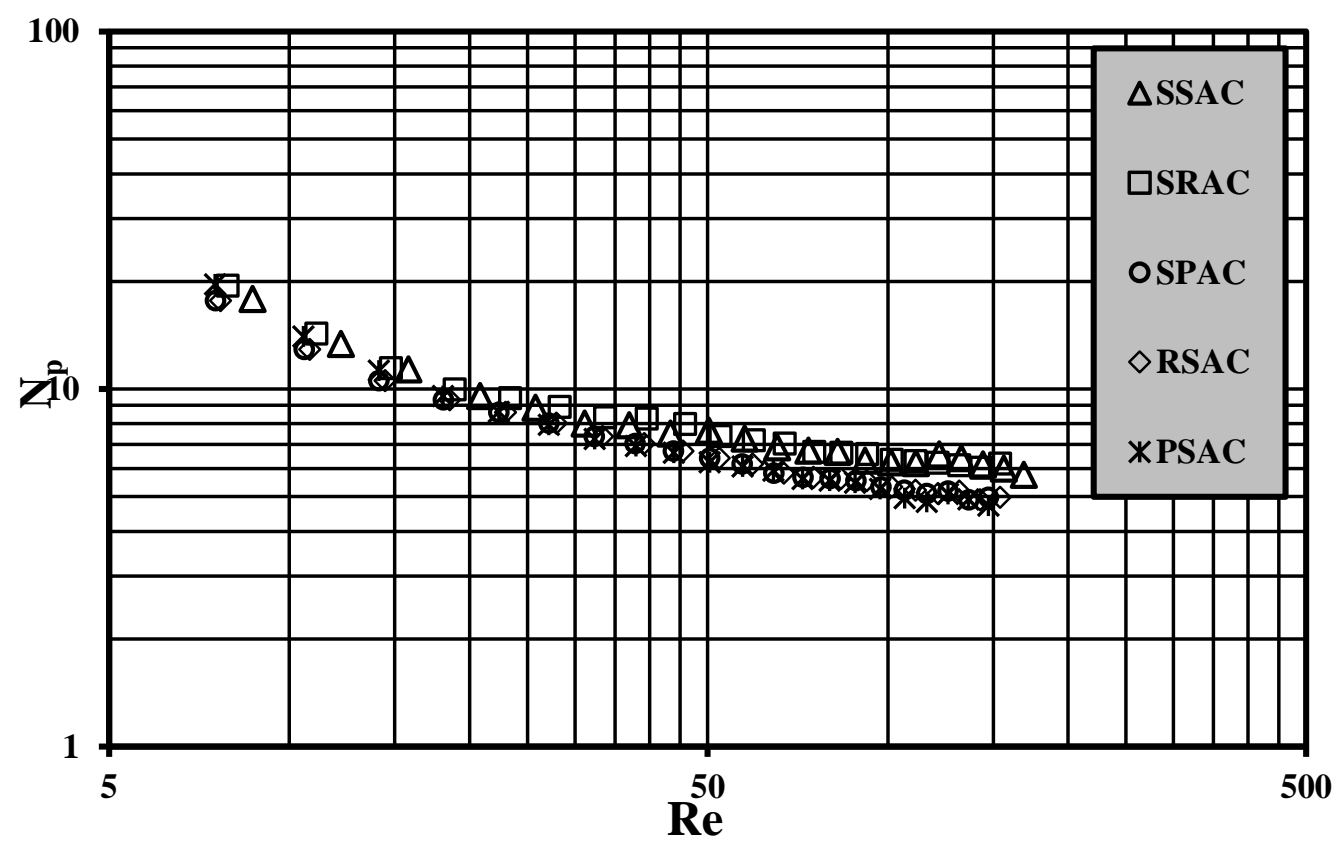

Figure (5.5-4). The master power curves for five coaxial mixers at $R_{n}=8,1.5 \%$ xanthan gum solution, and the co-rotating mode. 


\subsubsection{Flow Field}

In this section, the flow field produced by five coaxial mixers (i.e.SSAC, SRAC, RSAC, SPAC, and PSAC) are analyzed both qualitatively and quantitatively. Figure (5.5-5) illustrates the axial, radial, and tangential velocity contour plots of five coaxial mixers at $R_{n}=8\left(N_{c}=240 \mathrm{rpm}\right.$ and $\left.N_{a}=30 \mathrm{rpm}\right)$ in the laminar regime and the co-rotating mode

(a)
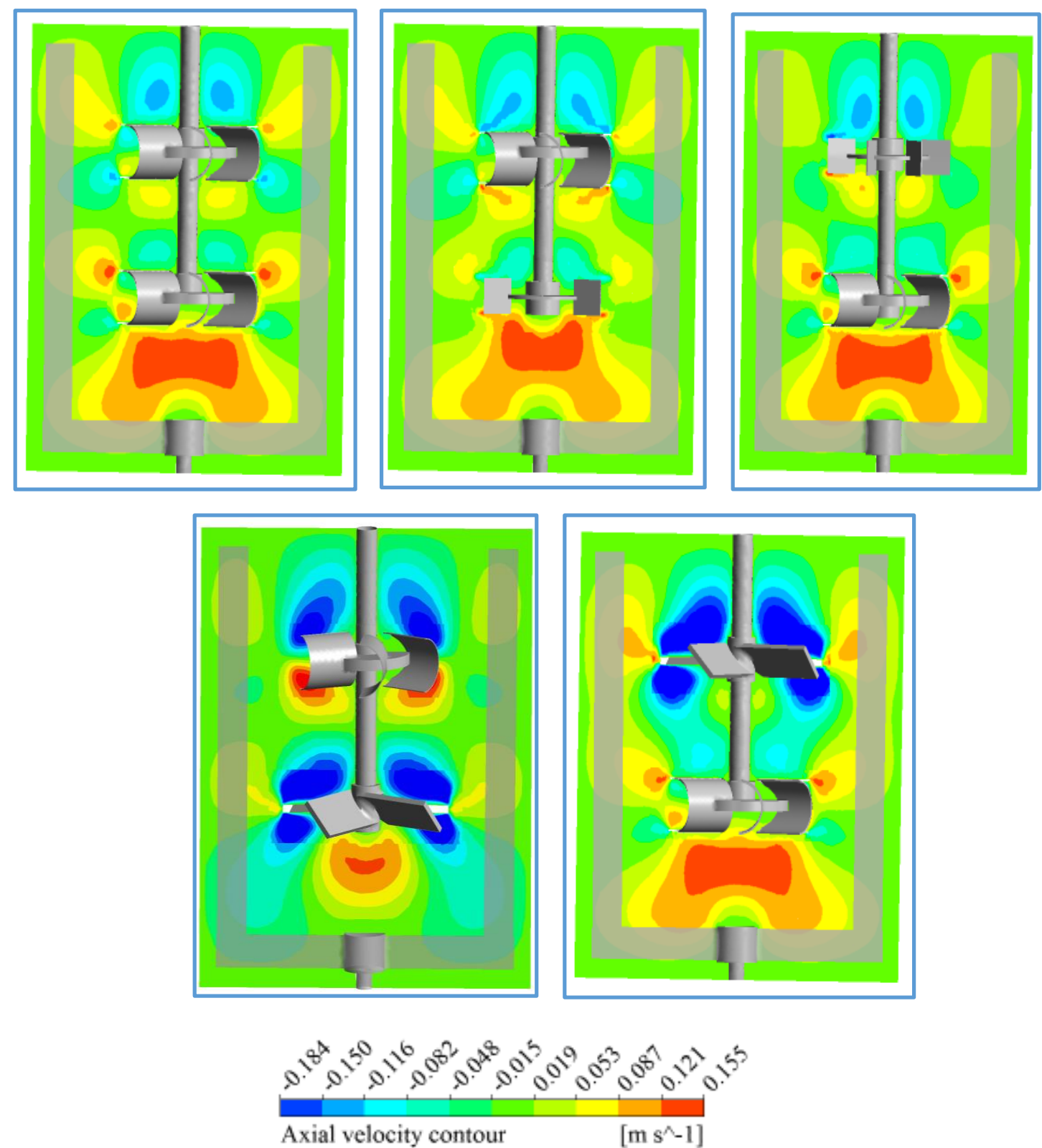
(b)
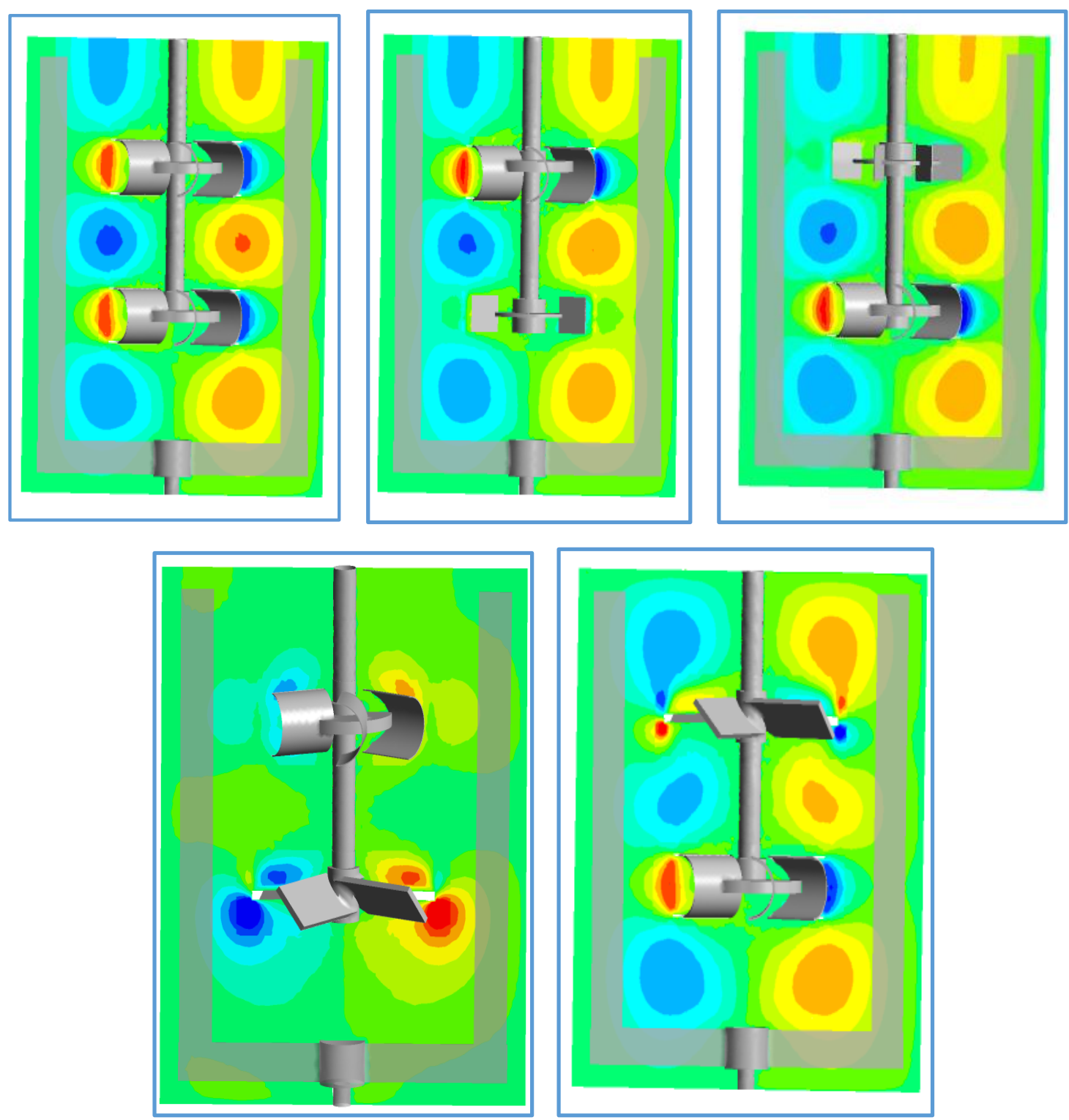

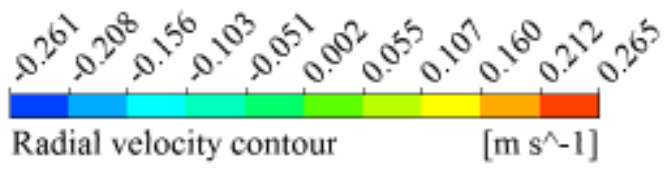


(c)
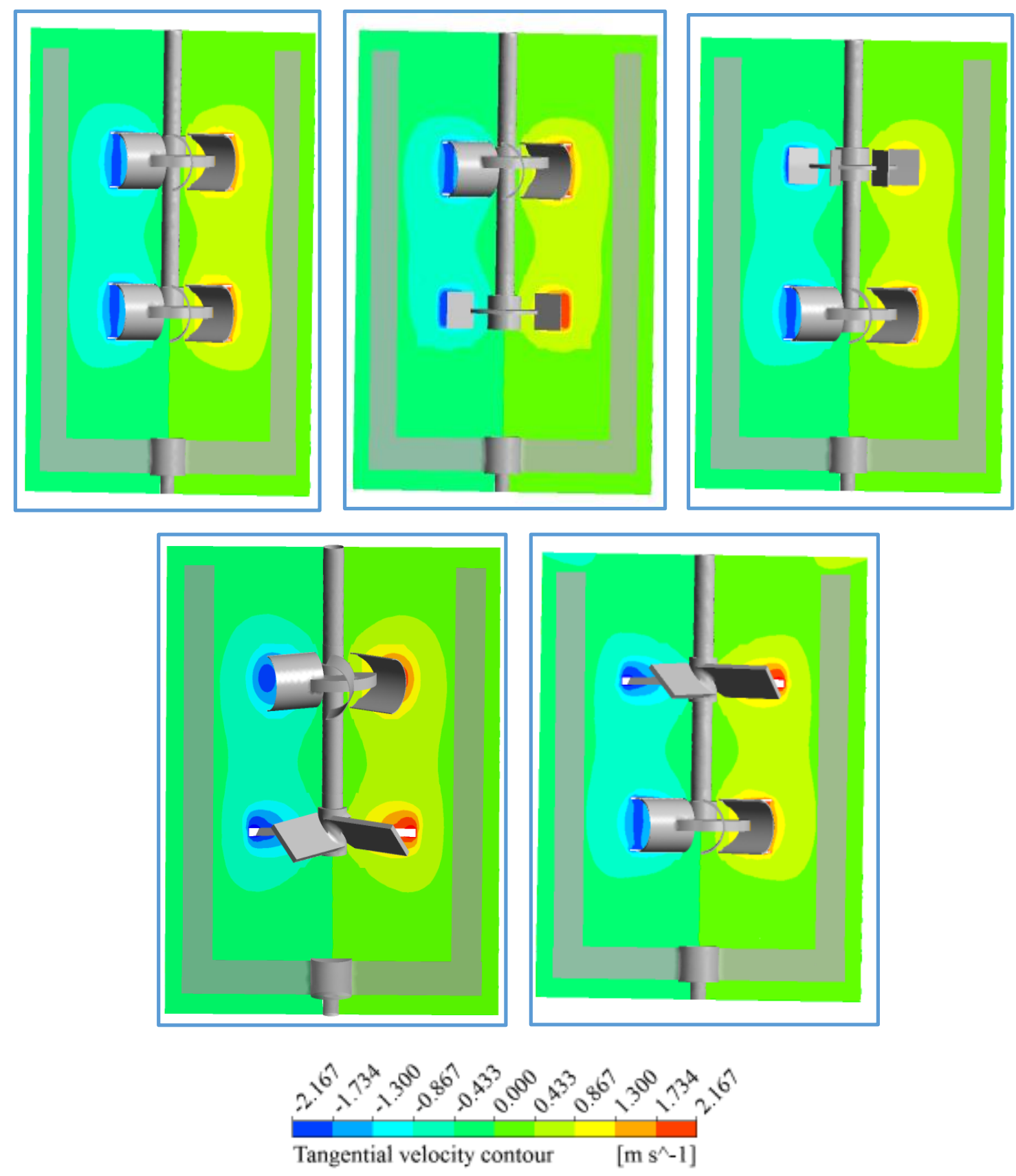

Figure (5.5-5). Velocity (m/s) contour plots at $R_{n}=8\left(N_{c}=240 \mathrm{rpm}\right.$ and $\left.N_{a}=30 \mathrm{rpm}\right)$ and $1.5 \%$ xanthan gum solution: (a) axial velocity, (b) radial velocity, and (c) tangential velocity. 
Figure (5.5-5) a shows that the maximum axial velocities were generated by the SPAC and PSAC configurations. As it can be noted for the SPAC mixer, both pitched blade impellers produced a maximum axial velocity of $V_{a}=0.184 \mathrm{~m} / \mathrm{s}$ in the upper and the lower parts of the tank. This resulted in a consistent distribution of the axial velocity inside of the tank. However, for the PSAC mixer, the pitched blade and Scaba impellers generated the maximum axial velocities of $V_{a}=0.184 \mathrm{~m} / \mathrm{s}$ and $V_{a}=0.155 \mathrm{~m} / \mathrm{s}$ in the upper part and the lower part of the tank, respectively. This distribution of the axial velocity profile was not consistent compared to the previously discussed configuration.

The radial velocity contours of five investigated coaxial mixers are presented in Figure ( 5.5-5). It can be seen that the maximum radial velocity of $V_{r}=0.265 \mathrm{~m} / \mathrm{s}$ was generated by the Scaba impeller in the SSAC, SRAC, RSAC, PSAC,and SPAC mixing system. These results also show that the maximum radial velocity of $V_{r}=0.265 \mathrm{~m} / \mathrm{s}$ was generated by the SPAC mixing system. Moreover, Figure (5.5-5) b demonstrates that the radial velocity patterns attained for both SRAC and RSAC were almost the same with some differences in the radial velocity magnitude.

The tangential velocity contour plots of the investigated coaxial mixers for a range of the tangential velocity from $V_{t}=-2.167 \mathrm{~m} / \mathrm{s}$ to $V_{t}=2.167 \mathrm{~m} / \mathrm{s}$ are shown in Figure $(5.5-5) \mathrm{c}$. This figure shows that the tangential velocity patterns created by five coaxial mixers utilized in this study were almost the same with a slight difference for the SSAC mixer compared to the others.

For quantitative analysis of the flow field generated by the different coaxial mixing configurations, the axial profiles of the dimensionless axial, radial, and tangential 
velocities at four radial positions $(2 r / T=0.25,2 r / T=0.45,2 r / T=0.58$, and $2 r / T=0.68)$ along the tank wall were generated and shown in Figures [(5.5-6), (5.5-7) and (5.5-8)].

Figure (5.5-6) illustrates the axial profile of the axial velocity along the tank wall. It can be noticed from Figure (5.5-6) a PSAC mixer generated a better axial velocity distribution at the radial position of $2 r / T=0.25$ with the peak values of $V_{a}=0.06 V_{t i p}$ at $z / H=0.26$ and $V_{a}=0.12 V_{\text {tip }}$ at $z / H=0.80$. A similar axial velocity profile was attained by the PSAC mixer when the radial position changed from $2 r / T=0.25$ to $2 r / T=0.45$ (tip of the central impellers) with the pick values of $V_{a}=0.064 V_{\text {tip }}$ at $z / H=0.42$ and $V_{a}=$ $0.16 V_{\text {tip }}$ at $z / H=0.68$ as depicted in Figure (5.5-6) b. By changing the radial position from the tip of the central impellers towards the anchor impeller $(2 r / T=0.58)$, the highest axial velocity $\left(V_{a}=0.04 V_{t i p}\right)$ was achieved by the SPAC and the PSAC mixers compared to the others [Figure (5.5-6) c]. However, at the radial position of $2 r / T=0.68$, a better axial velocity distribution with a peak value of $V_{a}=0.05 V_{\text {tip }}$ at $z / H=0.42$ was obtained by the SPTAC mixer [Figure (5.5-6) d]. Overall, the results presented in Figure (5.5-6) show that the axial velocity profiles attained by both SPAC and PSAC mixers are more beneficial for the fluid mixing in the axial direction compared to the other configurations. 
(a)

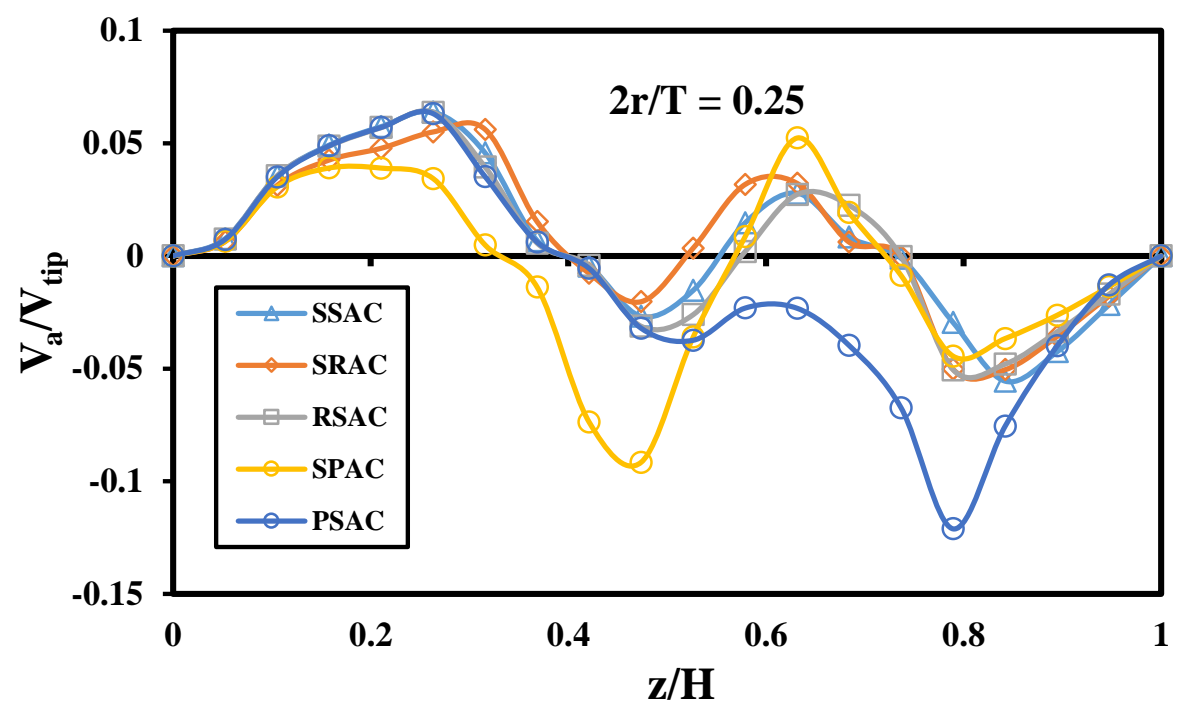

(b)

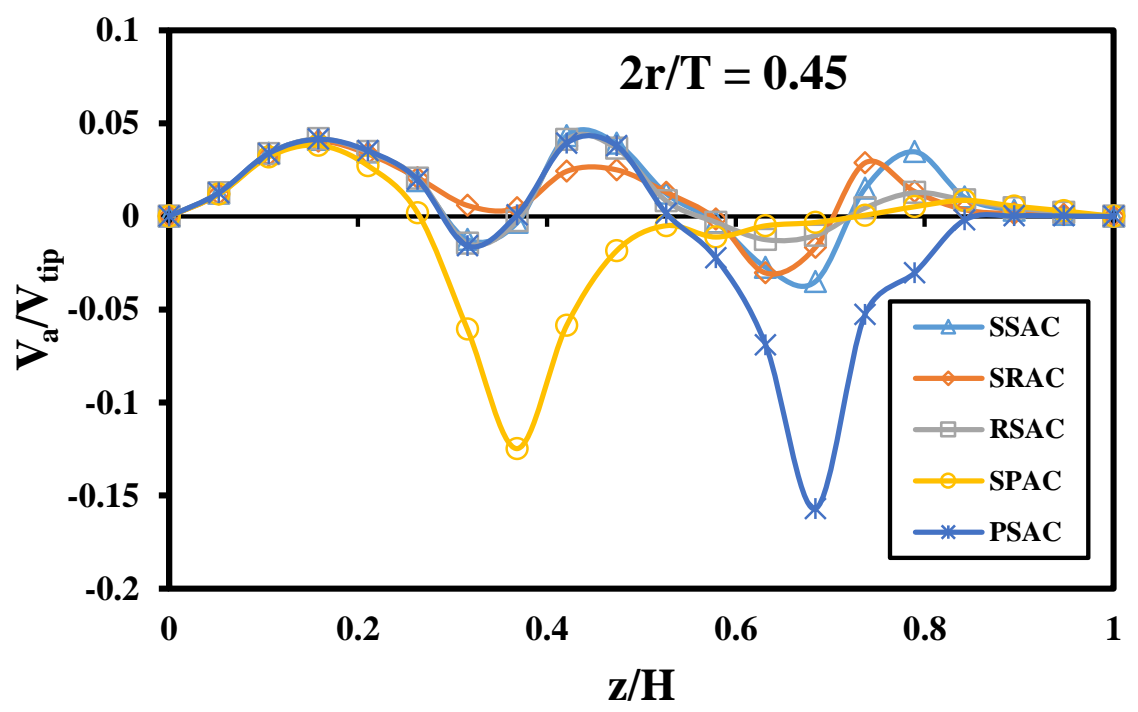


(c)

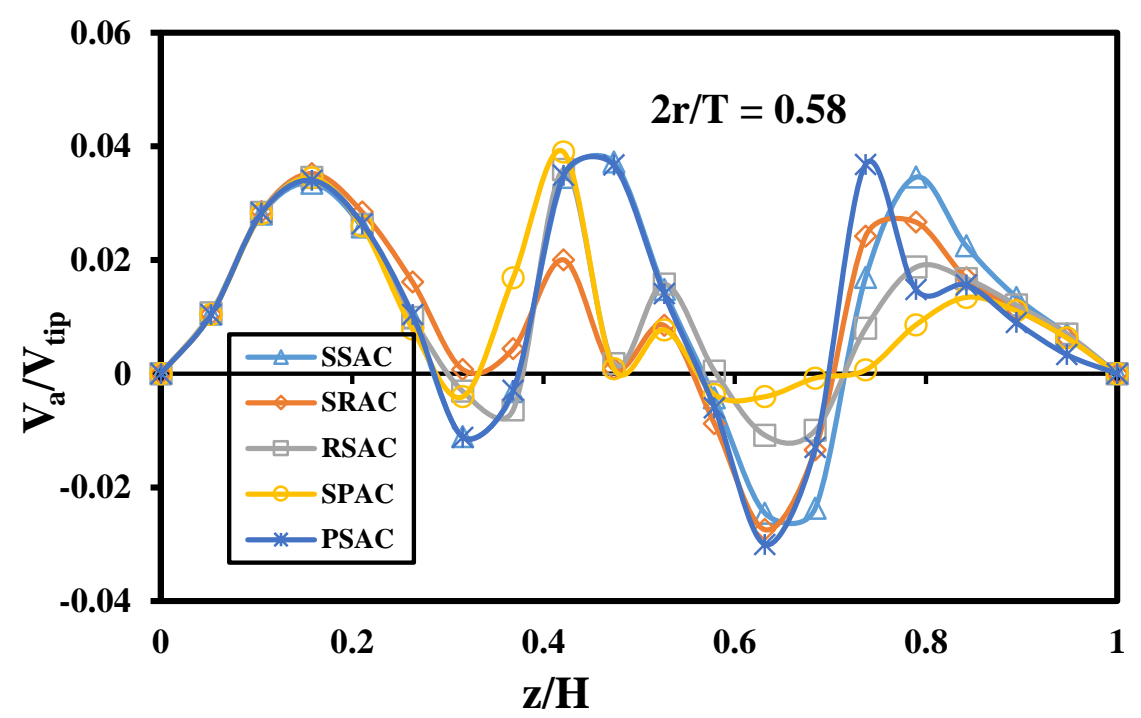

(d)

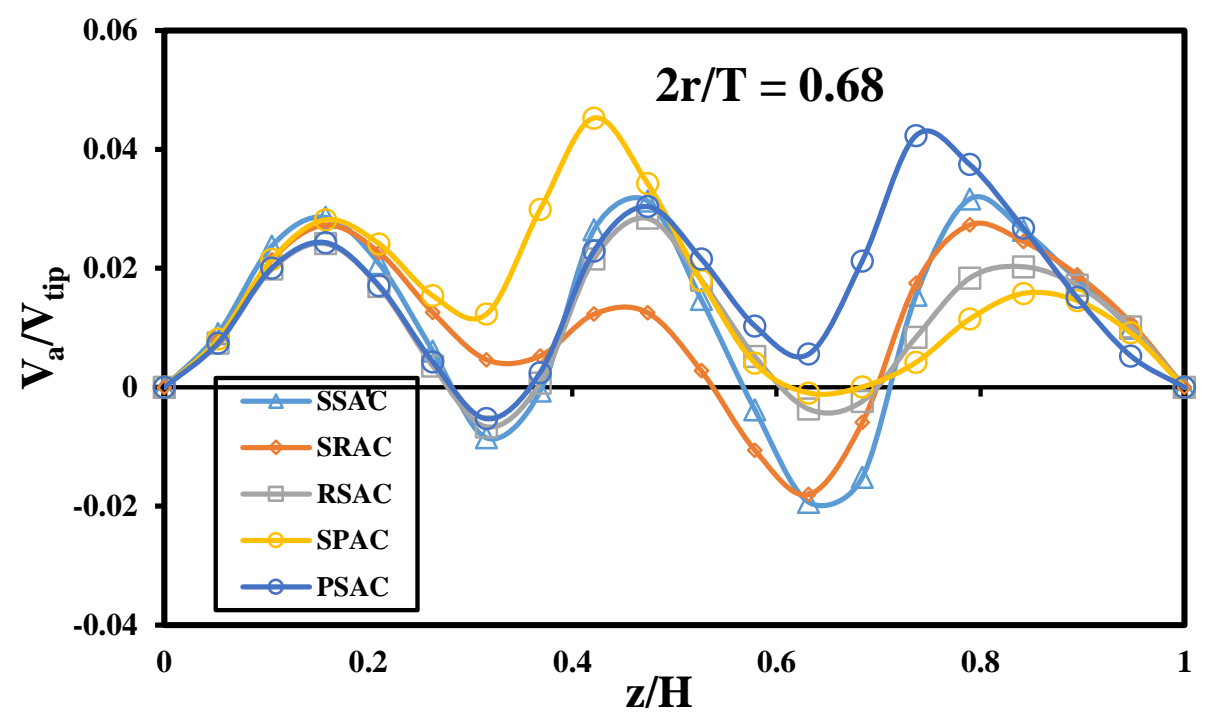

Figure (5.5-6). Normalized axial profiles of the axial velocity along the tank wall at $R_{n}=$ $8\left(N_{c}=240 \mathrm{rpm}\right.$ and $\left.N_{a}=30 \mathrm{rpm}\right)$ and $1.5 \%$ xanthan gum solution: (a) $2 r / T=0$, (b) $2 r / T$ $=0.45$, (c) $2 \mathrm{r} / \mathrm{T}=0.58$, and (d) $2 r / T=0.68$. 
Figure (5.5-7) depicts the axial profiles of the radial velocity along the tank height at four radial positions for five coaxial mixers analyzed in this study. It can be seen from Figure (5.5-7) a at the radial position of $2 r / T=0.25$, the PSAC mixer created a better axial profile of the radial velocity compared to the other configurations with the peak values of $V_{r}=0.05 V_{\text {tip }}$ at $z / H=0.74$ and $V_{r}=0.02 V_{\text {tip }}$ at $z / H=0.37$. By moving from the radial position of $2 r / T=0.25$ to the tip of the central impellers $(2 r / T=0.45)$, a better radial velocity distribution with a peak value of $V_{r}=0.16 V_{\text {tip }}$ at $z / H=0.37$ was achieved by the SPAC mixer as shown in Figure (5.5-7) b. In fact, in the SPAC mixing system, the pitched blade impeller acted as a radial impeller in the laminar flow with the highest radial velocity. This is in accordance with the studies conducted by Rodolph et al. (2007) and Kazemzadeh et al. (2016c) for the power-law and yield-pseudoplastic fluids. A similar profile was attained for the SPAC mixer, when the radial position changed from the tip of the central impellers $(2 r / T=0.45)$ to $2 r / T=0.58$ with a peak value of $V_{r}=0.07$ $V_{\text {tip }}$ at $z / H=0.37$ [Figure (5.5-7) c]. As depicted in Figure (5.5-7) d, at the radial position close to the anchor impeller $(2 r / T=0.68)$, it was found that the SSAC mixer generated a better radial velocity distribution compared to the others with a peak value of $V_{r}=0.07$ $V_{t i p}$ at $z / H=0.52$. Overall, the results presented in Figure (5.5-7) showed that the radial velocity profiles achieved by both SPAC and PSAC mixers were more beneficial for the radial fluid mixing at the impeller spacing equal or less than the central impeller diameter. 
(a)

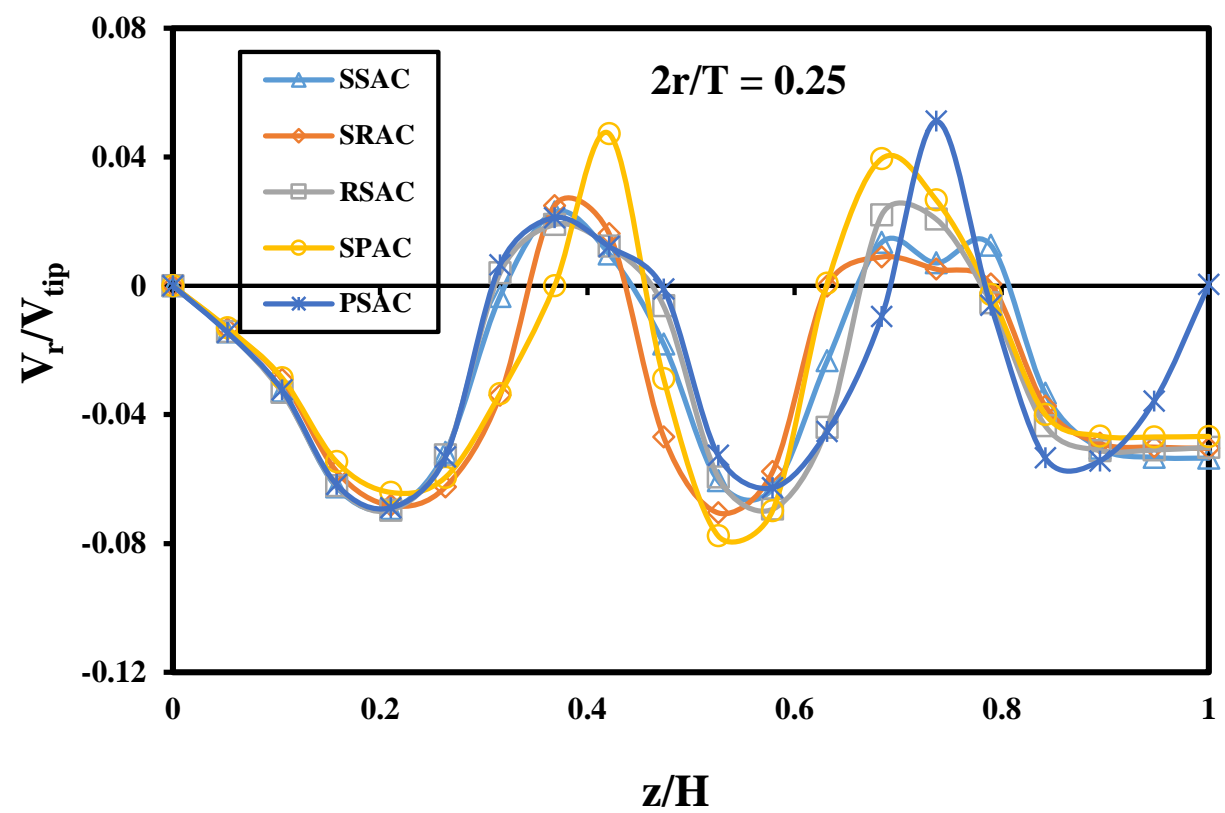

(b)

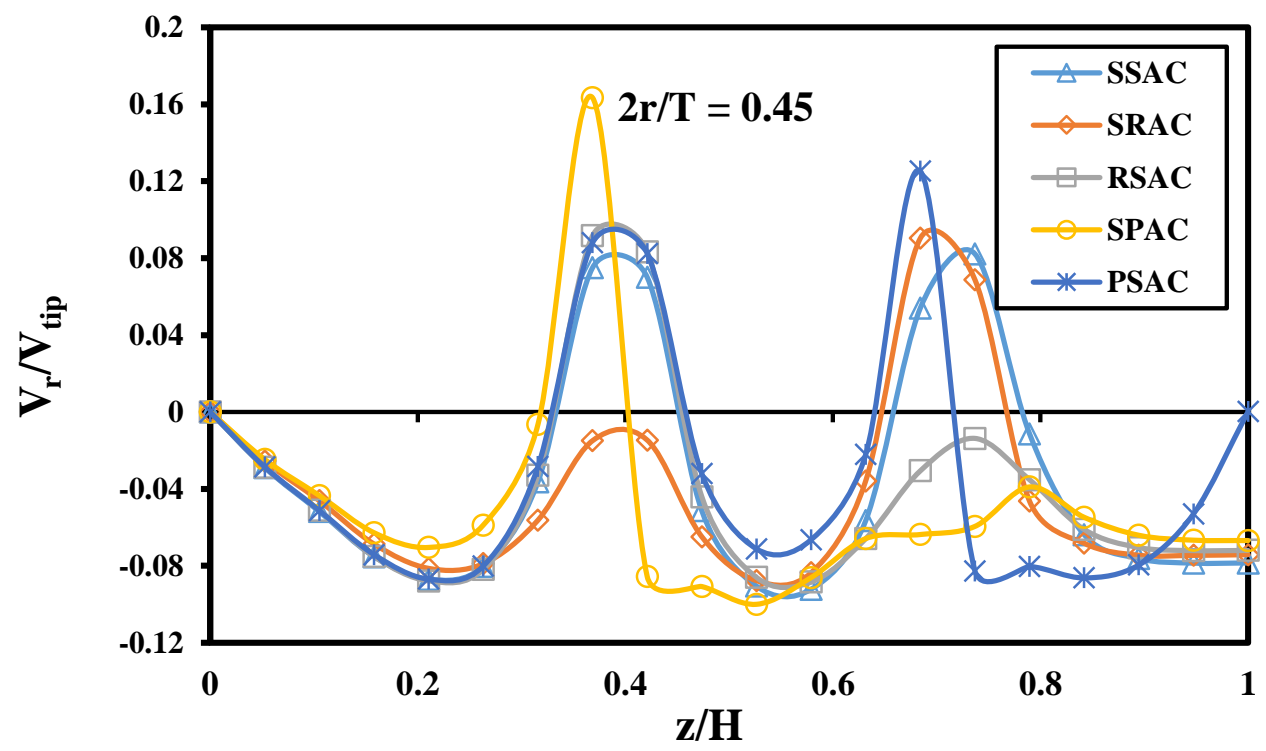


(c)

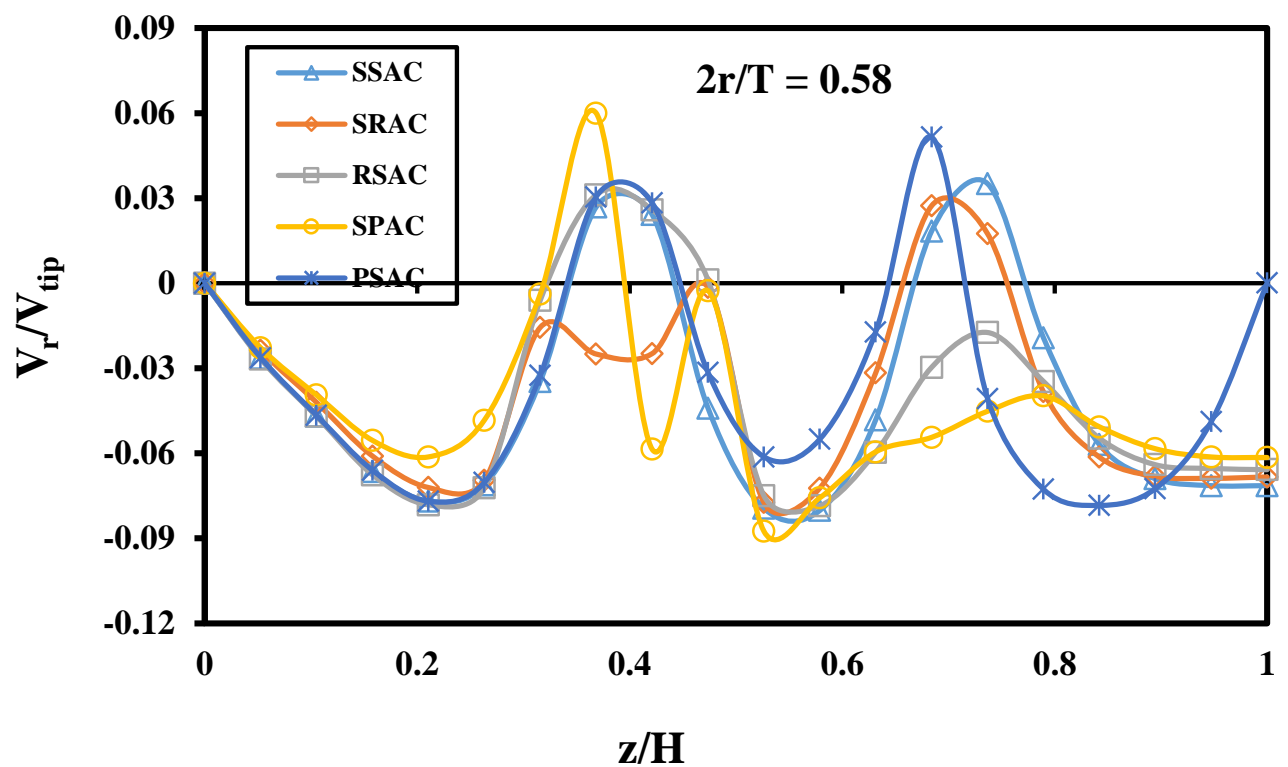

(d)

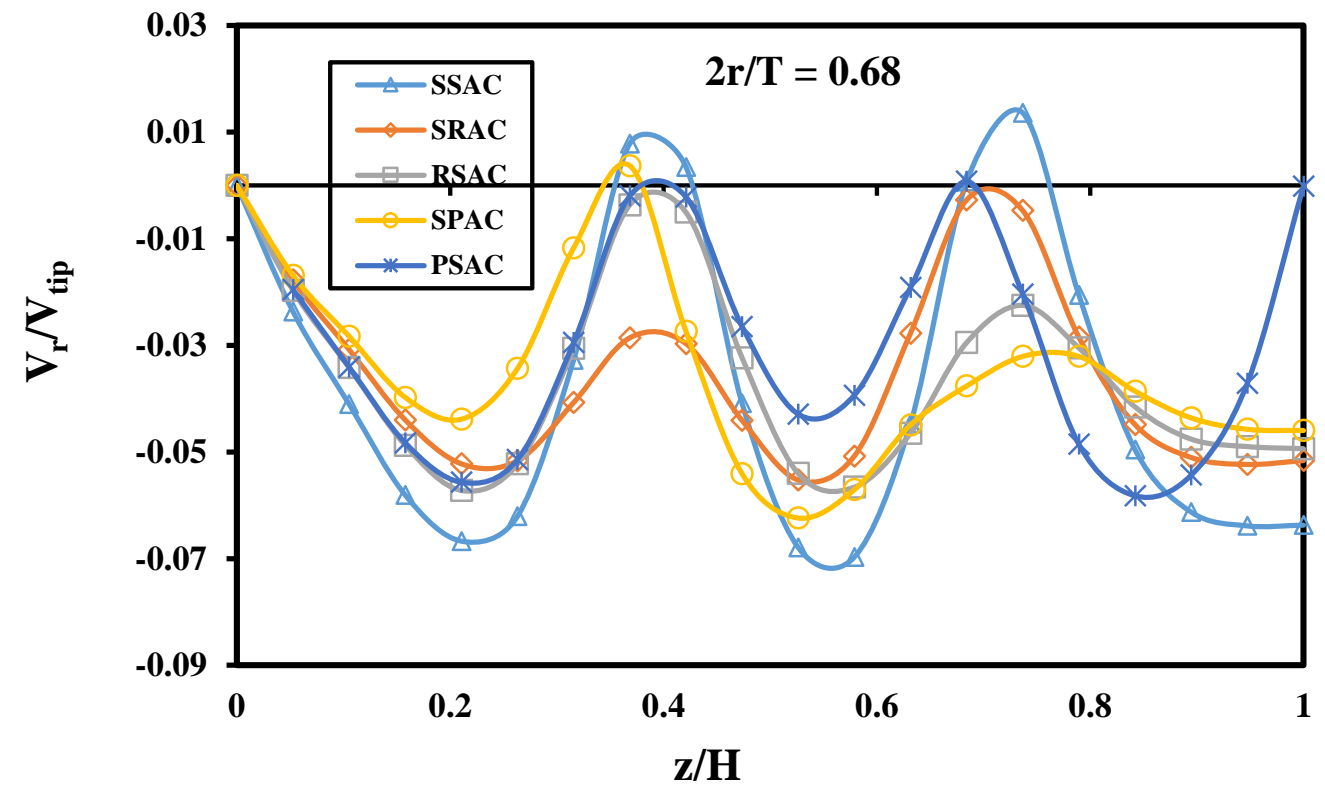

Figure (5.5-7). Normalized axial profiles of the radial velocity along the tank wall at $R_{n}=$ $8\left(N_{c}=240 \mathrm{rpm}\right.$ and $\left.N_{a}=30 \mathrm{rpm}\right)$ and $1.5 \%$ xanthan gum solution: (a) $2 r / T=0.25$, (b) $2 r / T=0.45$, (c) $2 r / T=0.58$, and (d) $2 r / T=0.68$. 
Figure (5.5-8) shows the axial profiles of the tangential velocity for five investigated coaxial mixers along the tank wall at four radial positions at $R_{n}=8\left(N_{c}=240 \mathrm{rpm}\right.$ and $N_{a}$ $=30 \mathrm{rpm})$. As it can be seen in Figure (5.5-8) a, all coaxial mixing configurations almost generated almost a similar tangential velocity distribution at the radial position of $2 r / T=$ 0.25 . When the radial position changed from $2 r / T=0.25$ to the tip of the central impellers $(2 r / T=0.45)$, the highest tangential velocities of $V_{t}=0.68 V_{\text {tip }}$ at $z / H=0.73$ and $V_{t}=0.63$ $V_{t i p}$ at $z / H=0.37$ were attained by the PSAC and the SPAC mixers, respectively. Moreover, at the radial position of $2 r / T=0.58$, the maximum tangential velocity was achieved by the SSAC, PSAC, and SPAC coaxial mixers. However, at the radial position near the anchor impeller $(2 r / T=0.68)$, a better tangential velocity distribution with the peak values of $V_{t}=0.24 V_{\text {tip }}$ at $z / H=0.42$ and $V_{t}=0.25 V_{\text {tip }}$ at $z / H=0.74$ was obtained by the SSAC mixer. Overall, it can be concluded that both PSAC and SPAC mixers generated a better distribution of the tangential velocities among the investigated coaxial mixers. 
(a)

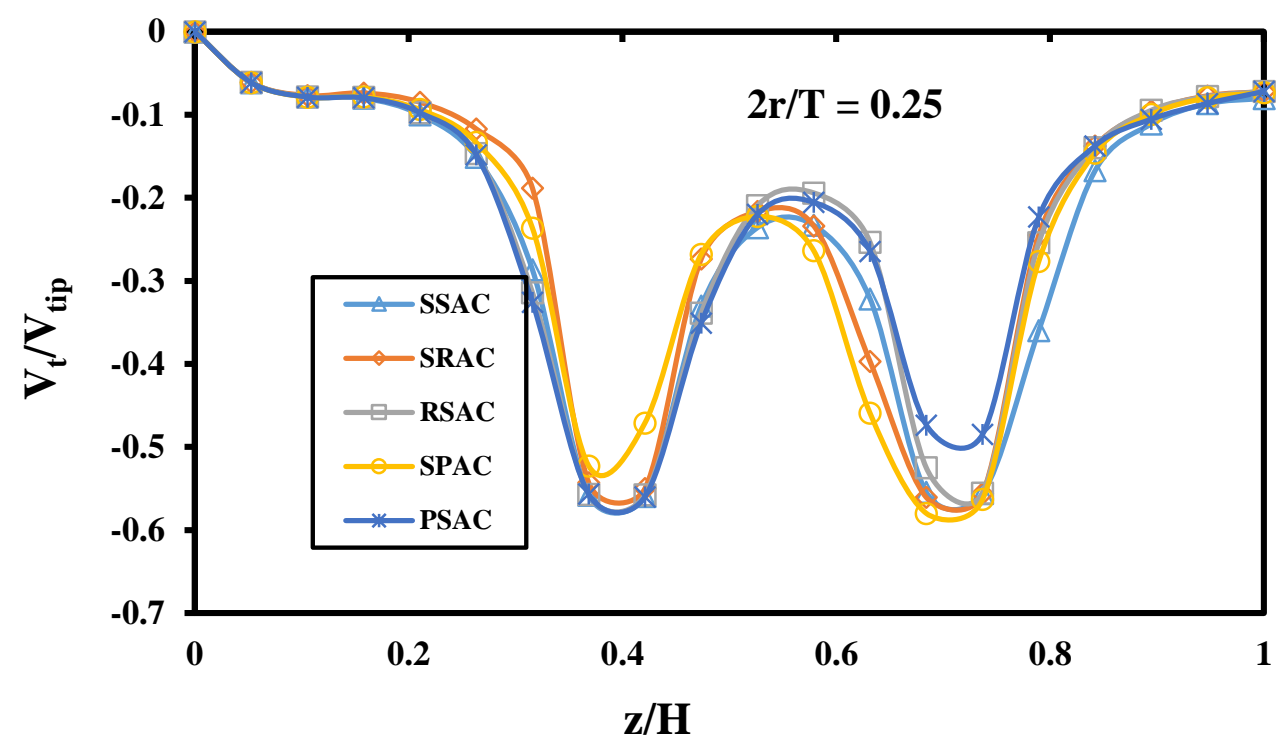

(b)

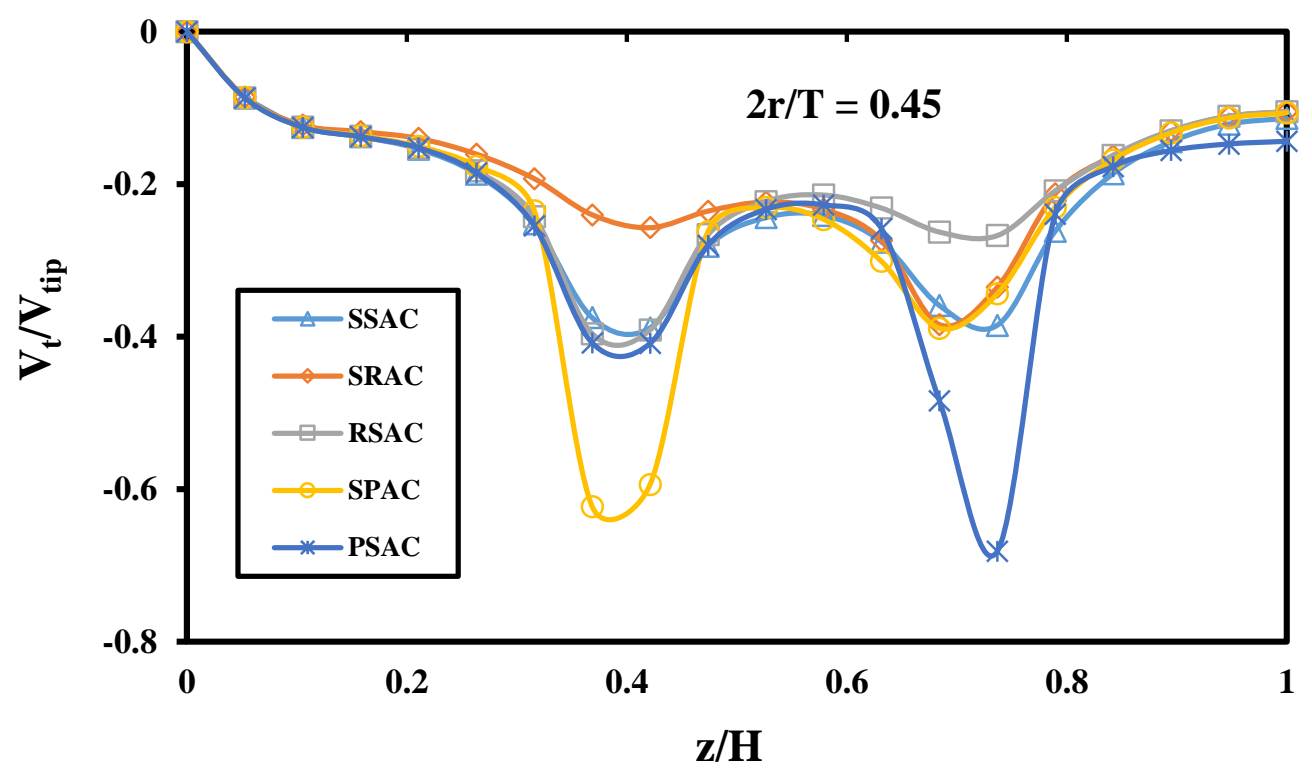


(c)

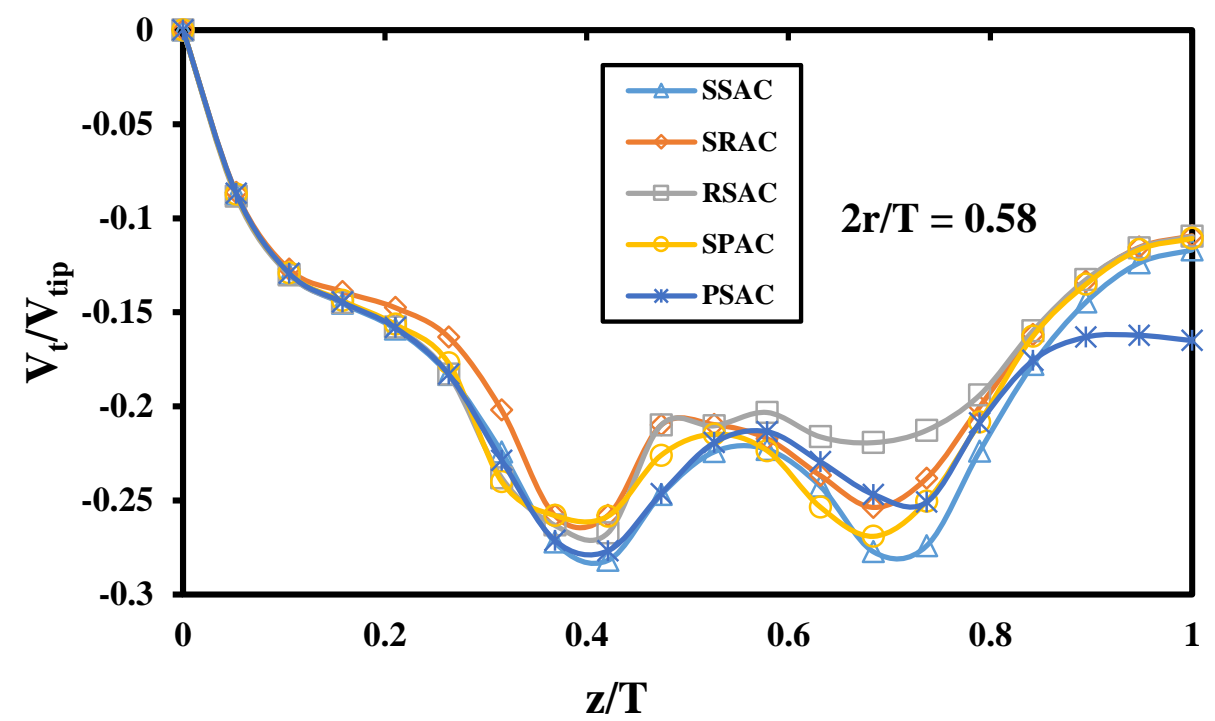

(d)

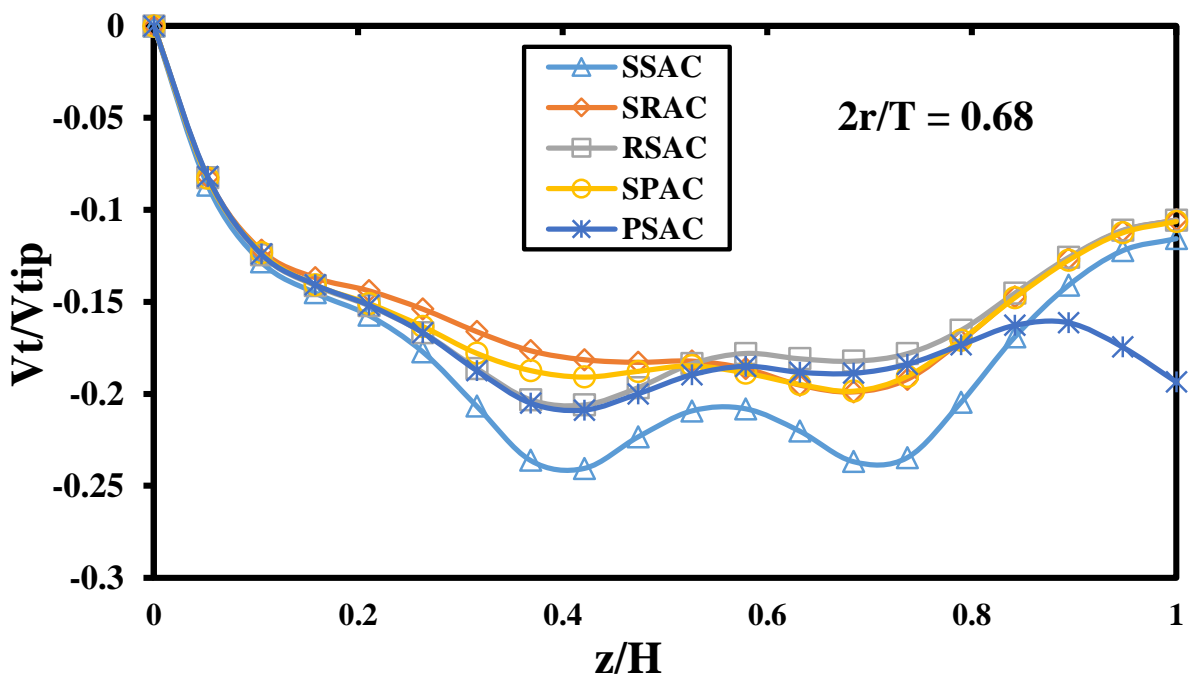

Figure (5.5-8). Normalized axial profiles of the tangential velocity along the tank wall at $R_{n}=8\left(N_{c}=240 \mathrm{rpm}\right.$ and $\left.N_{a}=30 \mathrm{rpm}\right)$ and $1.5 \%$ xanthan gum solution: (a) $2 r / T=0.25$, (b) $2 r / T=0.45$, (c) $2 r / T=0.58$, and (d) $2 r / T=0.68$. 
A comparative analysis of the radial profiles of the axial, radial, and tangential velocities at three axial positions $(z / H=0.2, z / H=0.50$, and $z / H=0.86)$ at $R_{n}=8\left(N_{c}=240 \mathrm{rpm}\right.$ and $N_{a}=30 \mathrm{rpm}$ ) was also conducted to assess the performances of five coaxial mixers utilized in this study. Figure (5.5-9) shows the radial profiles of the axial velocities generated by different coaxial mixers at different axial locations. It can be noted that similar trends at each specified axial location were obtained for all coaxial mixing configurations. These data demonstrate that the maximum values of the axial velocities for all mixers were attained at the same position $(2 r / T=0.26)$ but with different axial velocity magnitudes. However, the axial velocities decreased when the radial position moved from the central impellers toward the anchor impeller. The quantitative analysis of the data indicated that the SPAC mixer generated the maximum axial velocity of $V_{a}=$ $0.14 V_{\text {tip }}$ at $z / H=0.50$ as shown in Figure (5.5-9) b.

(a)

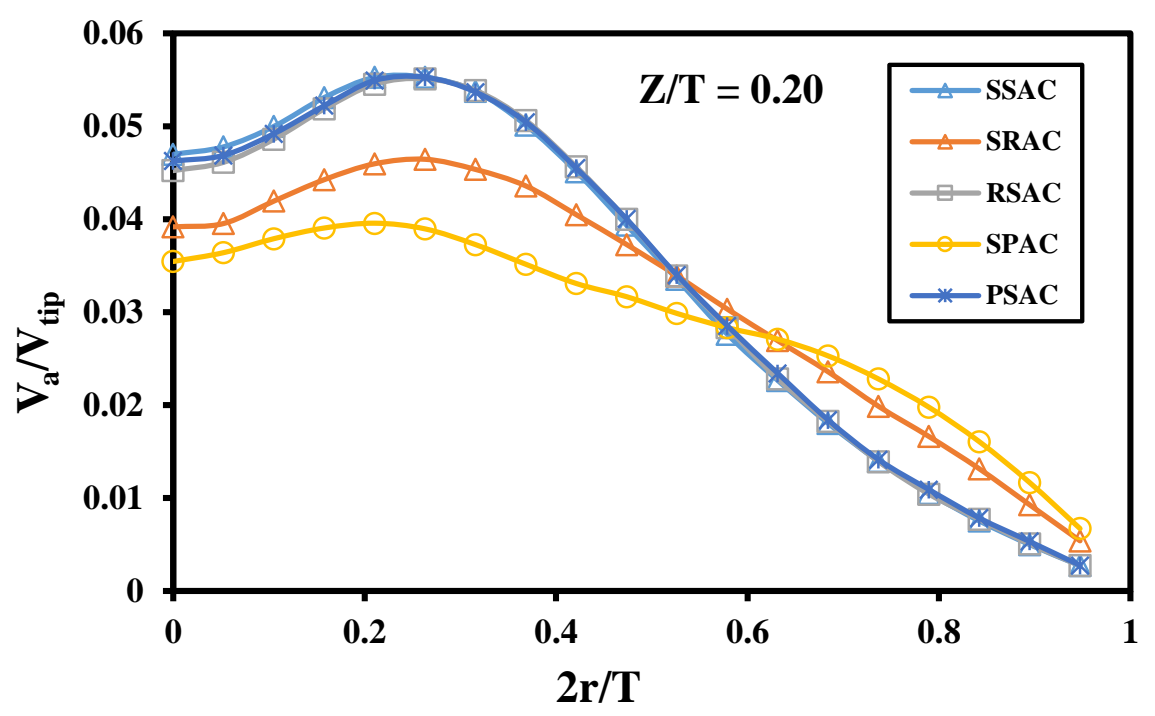


(b)

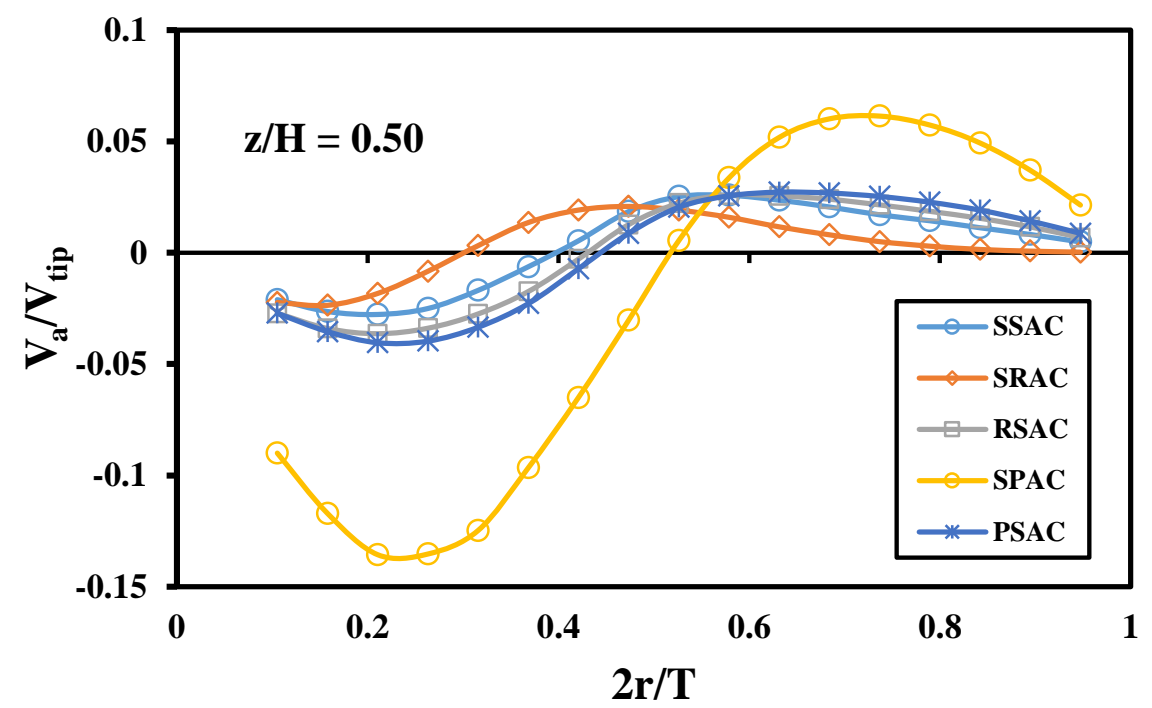

(c)

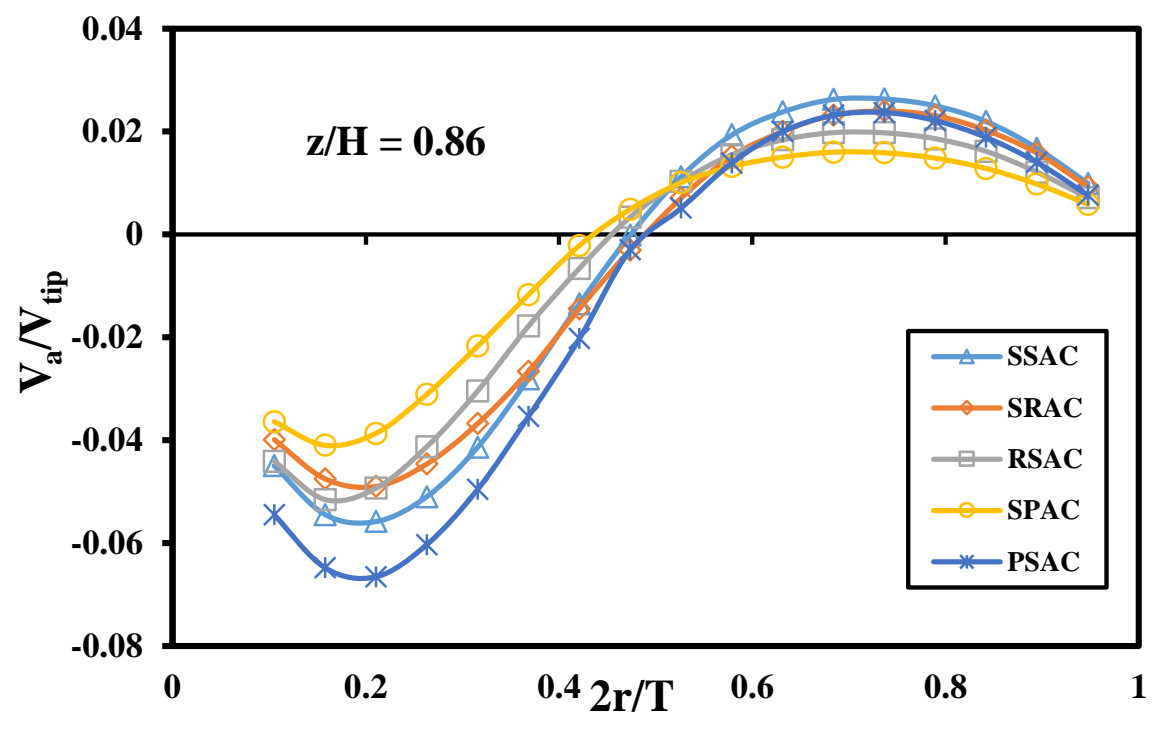

Figure (5.5-9). Normalized radial profiles of the axial velocity along the tank wall at $R_{n}=$ $8\left(N_{c}=240 \mathrm{rpm}\right.$ and $\left.N_{a}=30 \mathrm{rpm}\right)$ and $1.5 \%$ xanthan gum solution: (a) $z / H=0.20$, (b) $z / H=0.50$, and (c) $z / H=0.86$. 
Figure (5.5-10) depicts the radial profiles of the radial velocities for various coaxial mixing configurations. As it can be noticed, all coaxial mixers created similar radial velocity profiles with the maximum radial velocity at the radial position of $2 r / T=0.45$ when the axial position changed from $z / H=0.2$ to $z / H=0.86$. Further analysis revealed that at the bulk region between two impellers, Figure (5.5-10) b, the maximum radial velocity $\left(V_{r}=0.1 V_{t i p}\right)$ was achieved by the SPAC mixer at the axial position of $\mathrm{z} / \mathrm{H}=$ 0.50 .

(a)

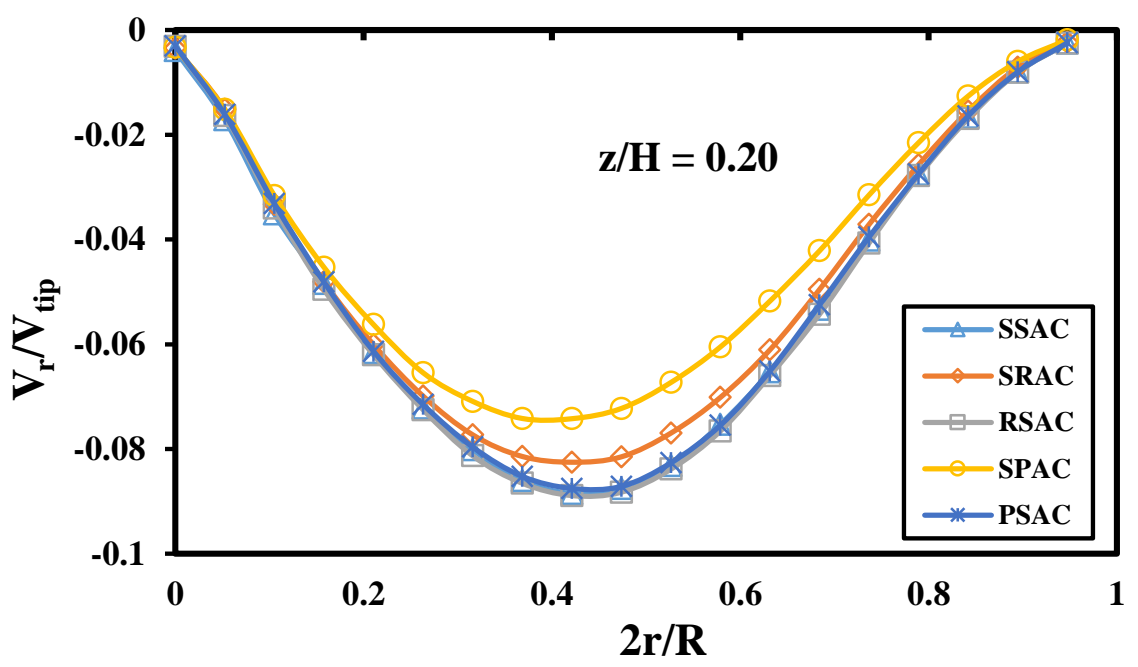


(b)

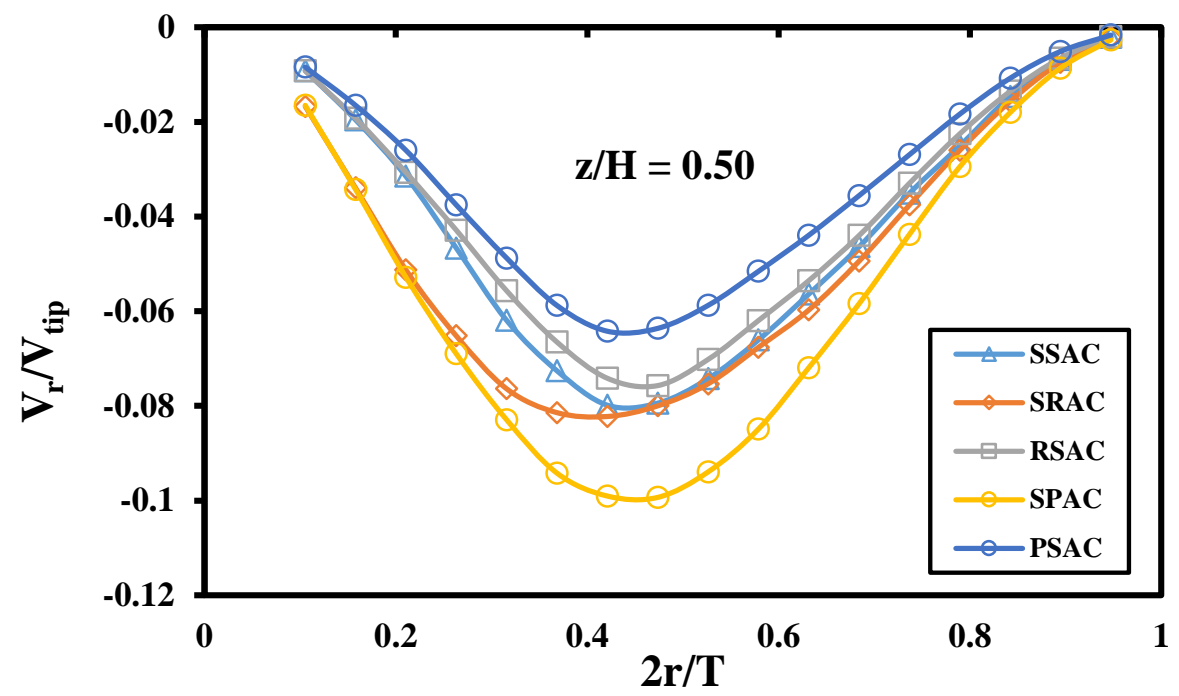

(c)

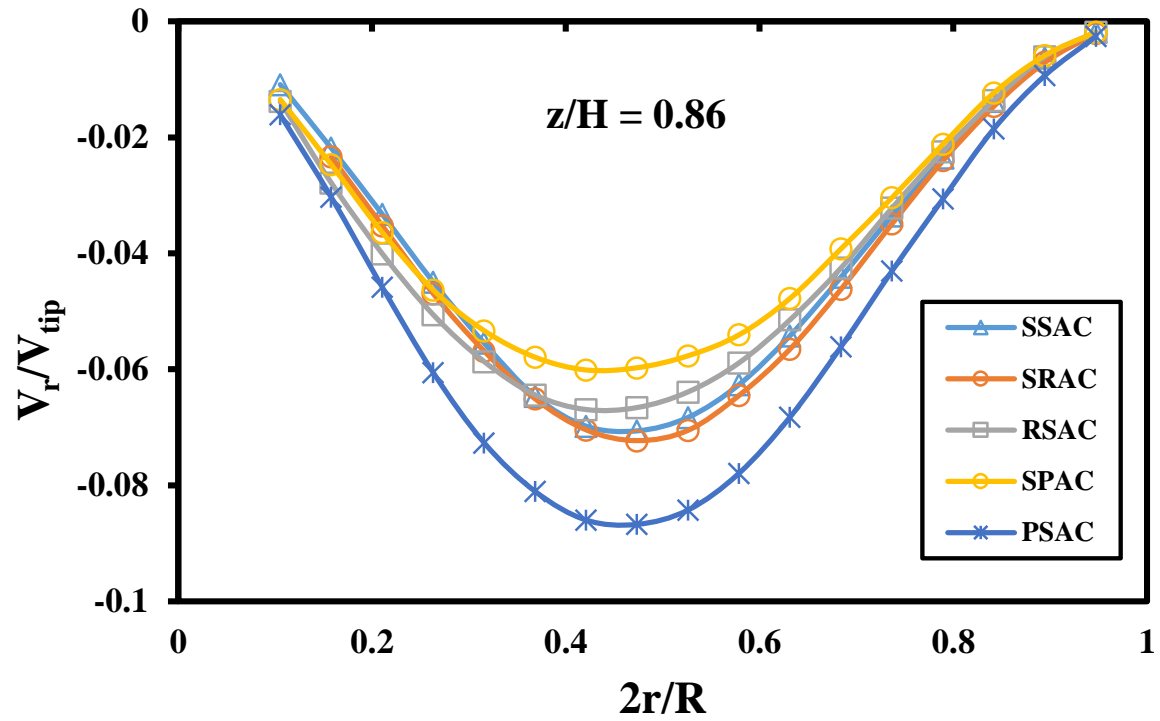

Figure (5.5-10). Normalized radial profiles of the radial velocity along the tank wall at $R_{n}$ $=8\left(N_{c}=240 \mathrm{rpm}\right.$ and $\left.N_{a}=30 \mathrm{rpm}\right)$ and $1.5 \%$ xanthan gum solution: (a) $z / H=0.20$, (b) $z / H=0.50$, and (c) $z / H=0.86$. 
The calculated radial profilers of tangential velocities at three axial positions are shown in Figure (5.5-11) for all coaxial mixers. These plots illustrate that the tangential velocity profiles were similar for all configurations when the axial position changed for $0.2<z / H$ $<0.86$. The maximum tangential velocity was observed around the radial positon of $2 r / T$ $=0.45$ and then the tangential velocity decreased when the radial position moved from the tip of the central impeller $(2 r / T=0.45)$ towards the anchor impeller.

(a)

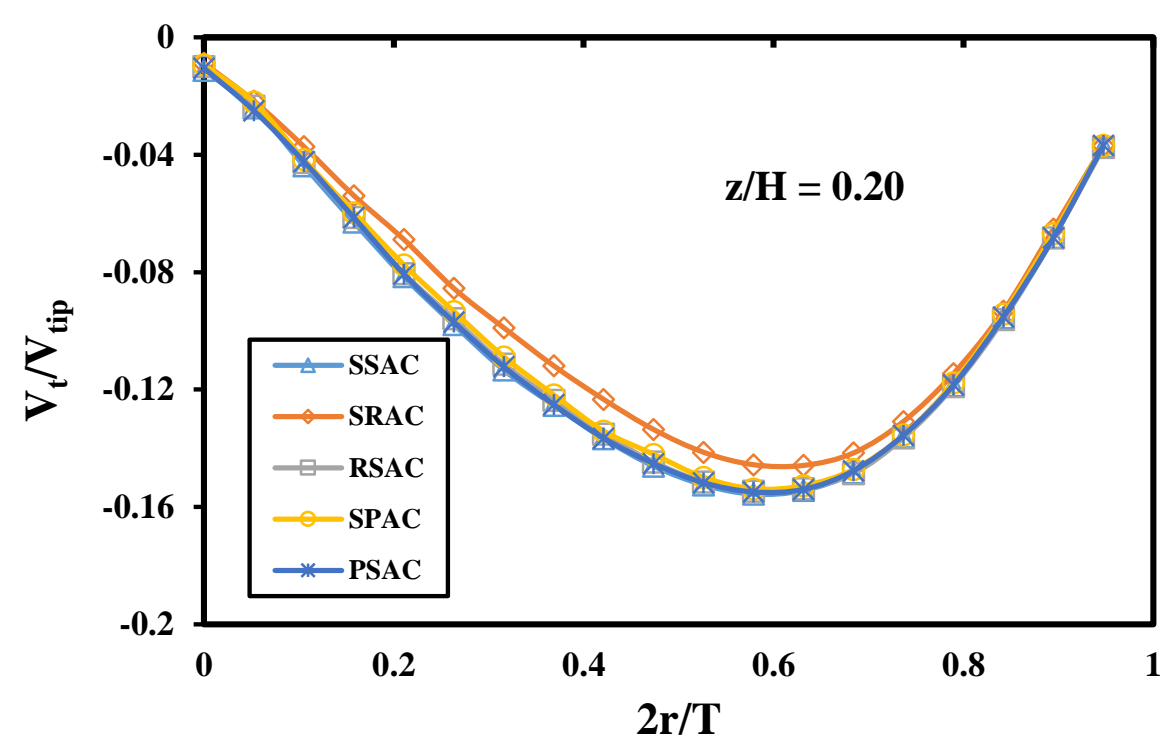


(b)

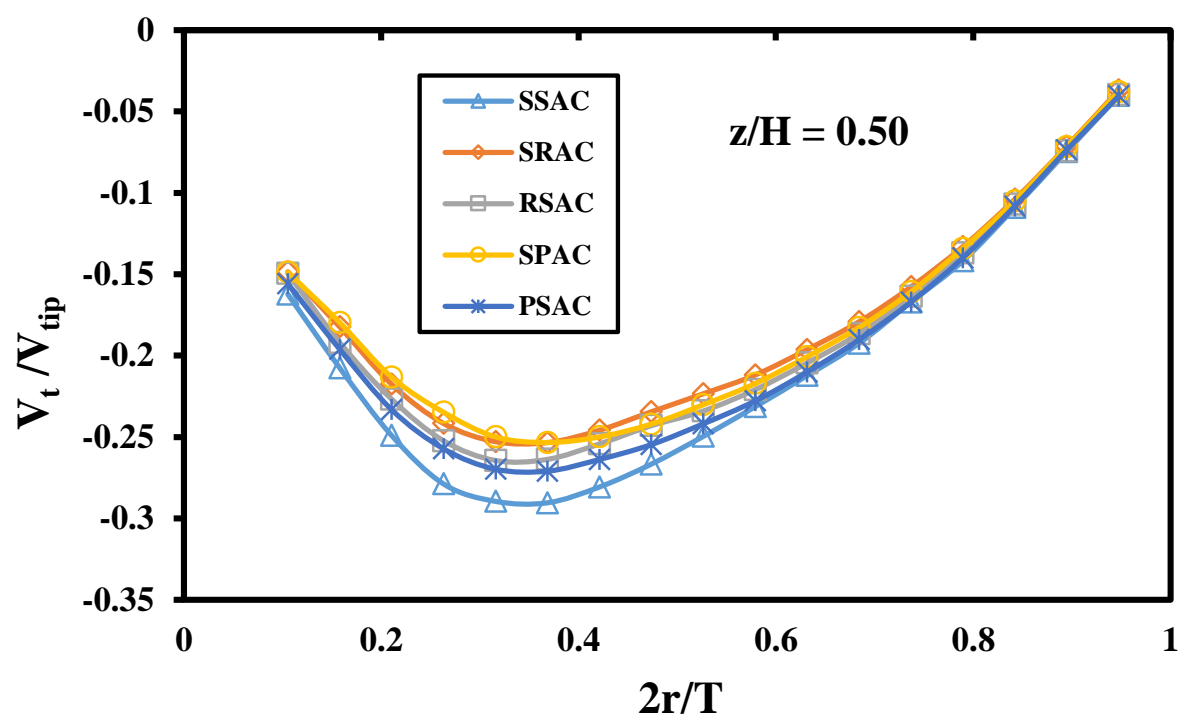

(c)

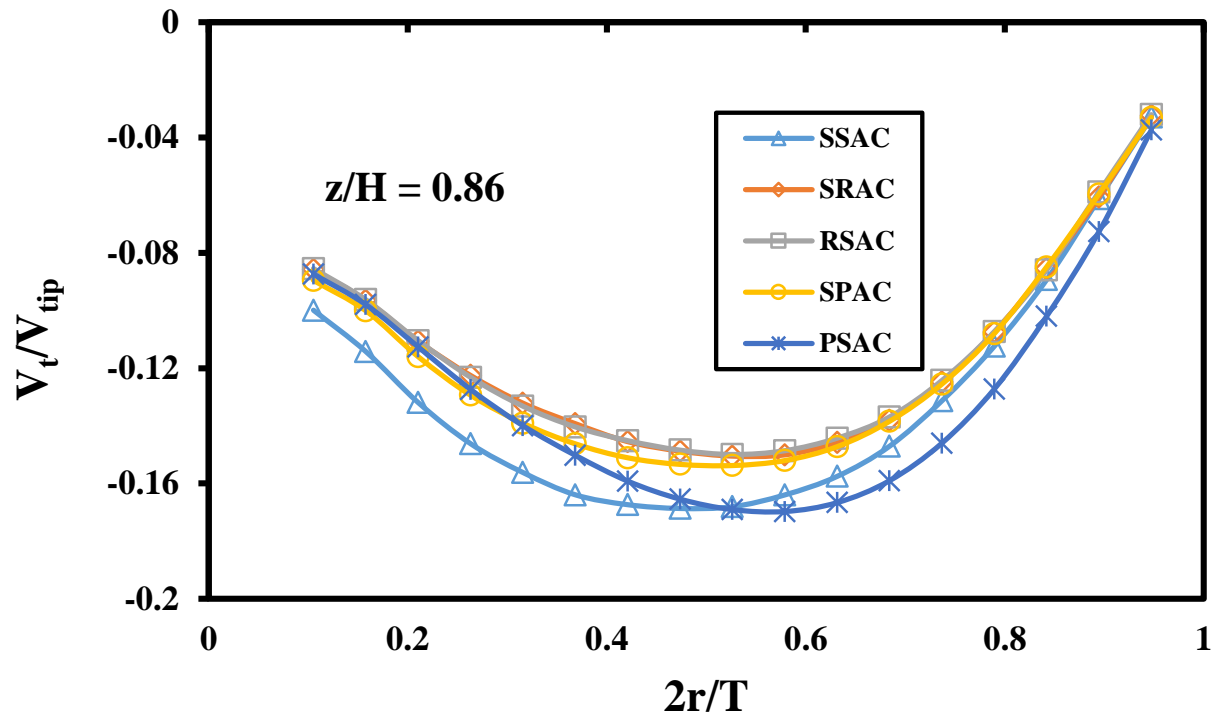

Figure (5.5-11). Normalized radial profiles of the tangential velocity along the tank wall at $R_{n}=8\left(N_{c}=240 \mathrm{rpm}\right.$ and $\left.N_{a}=30 \mathrm{rpm}\right)$ and $1.5 \%$ xanthan gum solution: (a) $z / H=$ 0.20, (b) $z / H=0.50$, and (c) $z / H=0.86$. 
To analyze the field, the normalized shear rates created by five coaxial mixers at four radial positions $(2 r / T=0.25,2 \mathrm{r} / / T=0.45,2 r / T=0.58$, and $2 r / T=0.68)$ along the tank wall at $R_{n}=8\left(N_{c}=240 \mathrm{rpm}, N_{a}=30 \mathrm{rpm}\right)$ are presented in Figure (5.5-12). This figure illustrates that the shear rates are higher in the regions between double central impellers and the anchor impeller. It was also found that the maximum shear rate $\left(\dot{\gamma} / N_{C o}=48\right)$ was achieved by the SPAC at $2 r / T=0.45$. Since the apparent viscosity of the xanthan gum solution depends on the shear rate, the viscosity contour plots were obtained using the CFD model at $R_{n}=8\left(N_{c}=240 \mathrm{rpm}, N_{a}=30 \mathrm{rpm}\right)$ as shown in Figure (5.5-13). This figure illustrates that the apparent viscosity inside the stirred tank was more uniform when the SSAC, SPAC, and SRAC mixers were employed. 
(a)

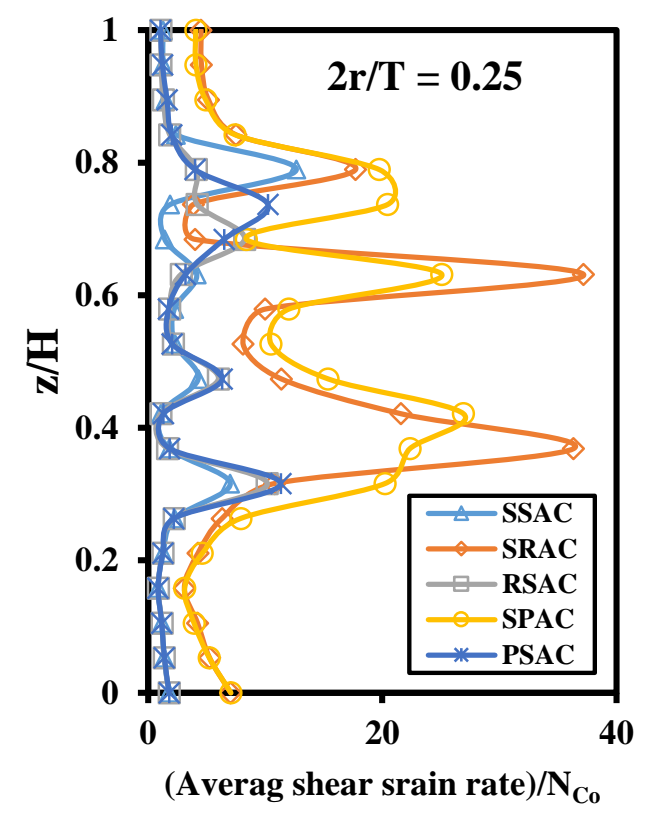

(c)

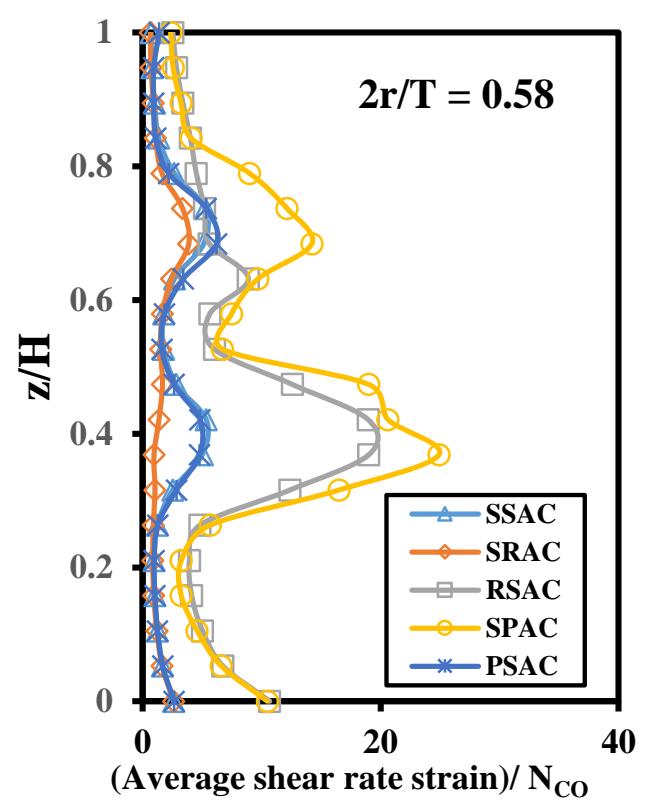

(b)

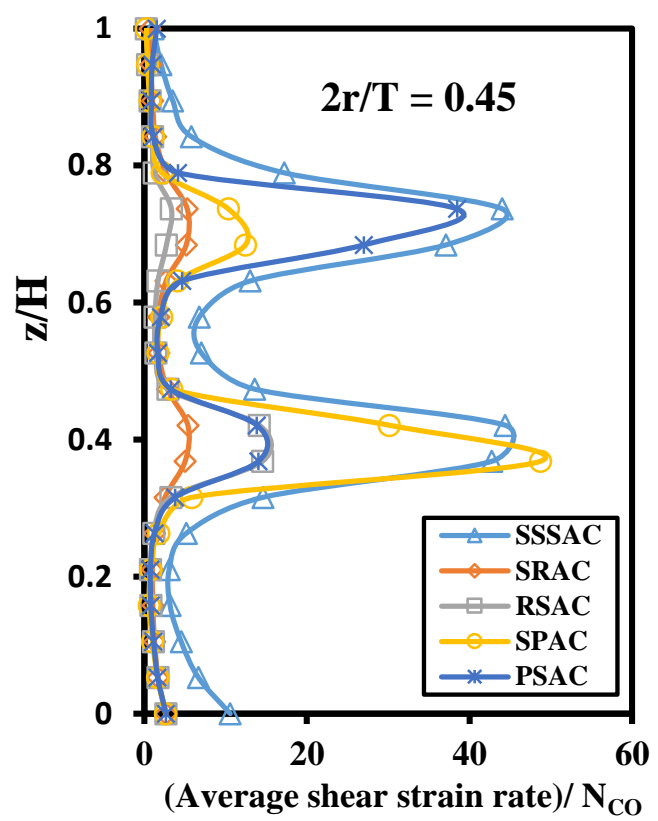

(d)

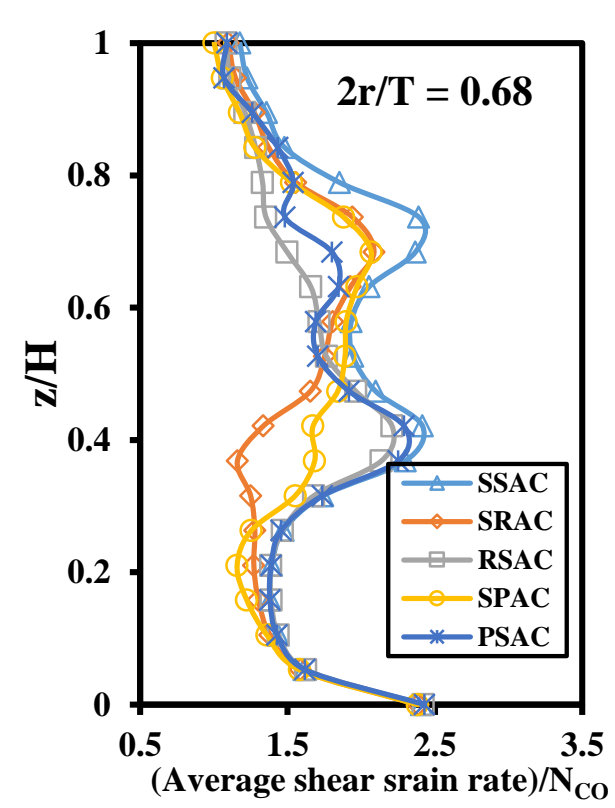

Figure (5.5-12). Dimensionless average shear rate at $R_{n}=8\left(N_{c}=240 \mathrm{rpm}\right.$ and $N_{a}=30$ $\mathrm{rpm}$ ) and $1.5 \%$ xanthan gum solution along a line parallel to the $z$-axis: (a) $2 r / T=0.25$, (b) $2 r / \mathrm{T}=0.45$, (c) $2 r / T=0.558$, and (d) $2 r / T=0.68$. 

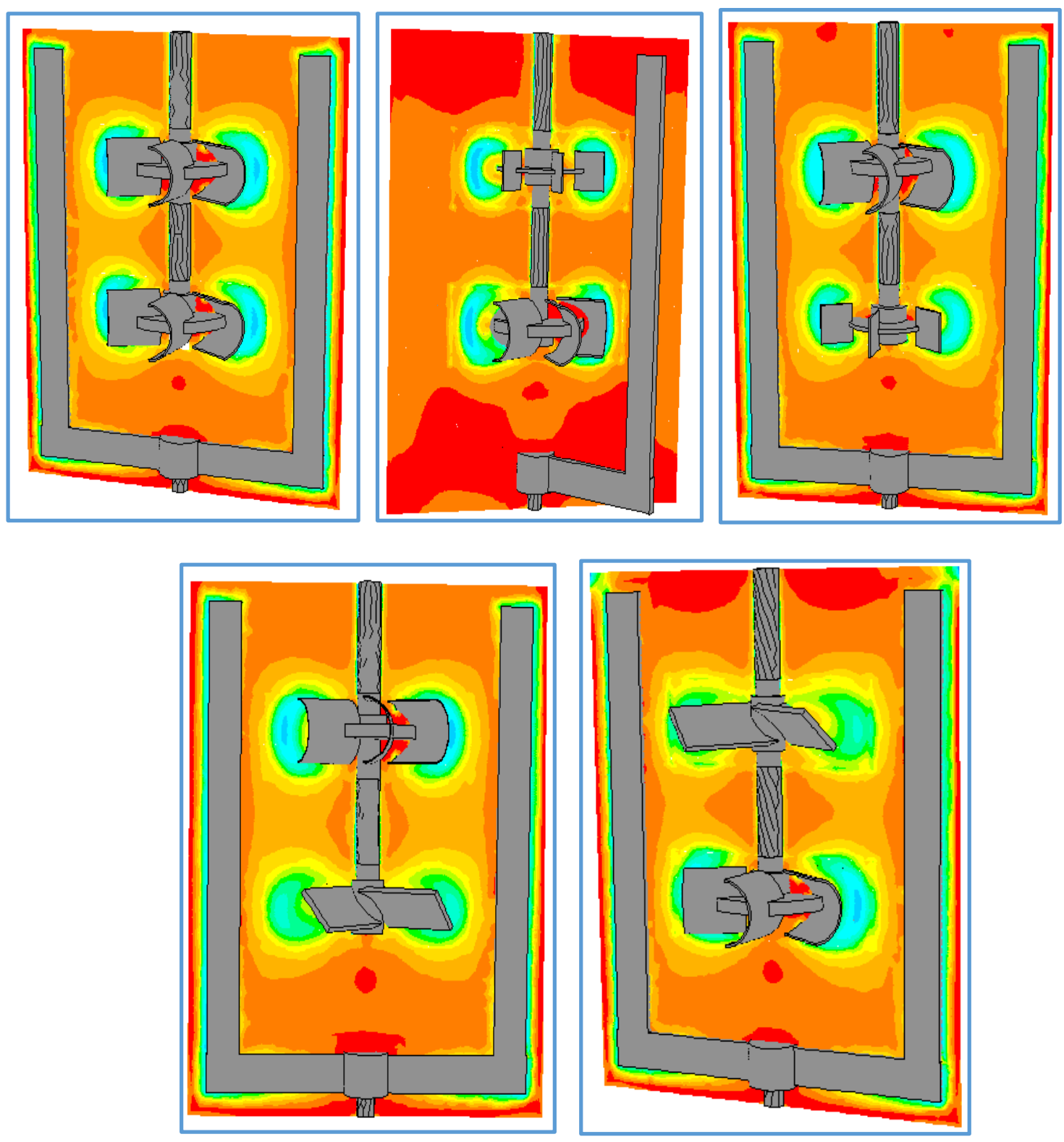

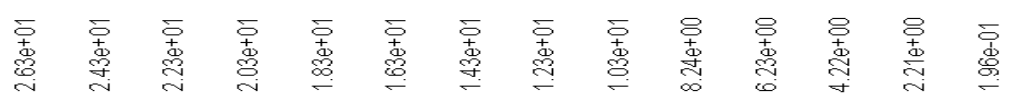

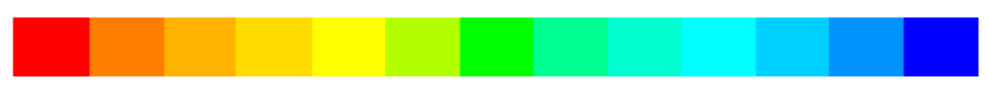

Figure (5.5-13). Apparent viscosity (kg/m.s) contour plots at $R_{n}=8\left(N_{c}=240 \mathrm{rpm}\right.$ and $N_{a}=30 \mathrm{rpm}$ ) and $1.5 \%$ xanthan gum solution: (a) Scaba-Scaba-anchor coaxial (SSAC) mixer, (b) Rushton-Scaba-anchor coaxial (RTSAC) mixer, (c) Scaba-Rushton-anchor coaxial (SRAC) mixer, (d) Scaba-pitched blade-anchor coaxial (SPAC) mixer, and (e) pitched blade-Scaba-anchor coaxial (PSAC) mixers. 


\subsubsection{Power Number, Pumping Number, Mixing Time, and Pumping Effectiveness}

For quantitative analysis, several parameters (power number, pumping number, mixing time, and pumping effectiveness) for various configurations of coaxial mixers explored in this study were calculated and compared at the same conditions. The power number of the coaxial mixers were calculated using Equation (5.5-1) at $R_{n}=8\left(N_{c}=240 \mathrm{rpm}\right.$ and $N_{a}$ $=30 \mathrm{rpm})$ and the corresponding Reynolds number of 170 was determined by using Equation (5.5-5). To compare the effectiveness of the investigated coaxial mixers, the pumping effectiveness was calculated as suggested by Mishra and Joshi (1994):

$$
\eta_{E}=\frac{N_{Q}}{N_{P}} \times 100
$$

In order to calculate the pumping effectiveness, the pumping capacity $(Q)$ and then the pumping number $\left(N_{Q}\right)$ must be calculated. The pumping capacity of the radial-flow impellers (i.e. the Scaba and Rushton impellers) was calculated by integrating the mean average radial velocity on a vertical plane at the tip of the impeller $\left(r=R_{i}\right)$ from the lower edge $(-z)$ to the upper edge $(+z)$ of the impeller. For the axial flow impeller (i.e. the pitched blade impeller), the pumping capacity was determined through the integration of the average axial velocities over a horizontal plane below the impeller blades from the hub to the radial position of D/2 (Pakzad et al., 2013a, Kazemzadeh et al., 2016c):

$$
\begin{aligned}
& Q_{(\text {Scaba, Rushton })}=\pi D_{c} \int_{-z}^{Z} \bar{V}_{r} d z \\
& Q(P B T)=2 \pi \int_{h u b}^{D c / 2} \bar{V}_{a} r d r
\end{aligned}
$$

The flow number was then defined by the following equation: 


$$
N_{Q}=\frac{Q}{N D_{S}{ }^{3}}
$$

Finally, the mixing times for all various configurations of the stirred system were measured through both experiments and simulations. The results of the above calculated parameters are summarized in Table (5.5-2). The results demonstrate that the lowest power numbers were obtained for the PSAC and SPAC mixers as $N_{p}=5.62$ and $N_{p}=$ 5.98 , respectively. In terms of the flow numbers, it was found that the flow number for the SSAC mixer $\left(N_{Q t}=0.63\right)$ was the highest compared to the other configurations. Both experimentally measured mixing time and computationally calculated mixing times revealed that the lowest mixing time was observed for the SPAC mixer system. A pumping effectiveness base analysis [Eq. (5.5-6)], showed that the efficiency of the SPAC mixer was the highest among the investigated configurations. These results confirm our previous findings that the SPAC mixer is an efficient mixer for the agitation of the yield pseudoplastic fluids.

Table (5.5-2). Power number, flow number, pumping effectiveness, and mixing time for five coaxial mixers at $R_{n}=8\left(N_{a}=30 \mathrm{rpm}\right.$ and $\left.N_{s}=240 \mathrm{rpm}\right), 1.5 \%$ xanthan gum solution, and co-rotating mode.

\begin{tabular}{ccccccc}
\hline $\begin{array}{c}\text { Double } \\
\text { Scaba- } \\
\text { anchor }\end{array}$ & $\begin{array}{c}\text { Power } \\
\text { number } \\
\left(N_{p}\right)\end{array}$ & $\begin{array}{c}\text { Flow } \\
\text { number } \\
\text { upper } \\
\text { impeller } \\
N_{Q u}\end{array}$ & $\begin{array}{c}\text { Flow } \\
\text { number } \\
\text { lower } \\
\text { impeller } \\
N_{Q l}\end{array}$ & $\begin{array}{c}\text { Total } \\
N_{Q t}\end{array}$ & $\begin{array}{c}\text { Mixing } \\
\text { time } \\
(\min )\end{array}$ & $\begin{array}{c}\text { Pumping } \\
\text { effectiveness } \\
\left(N_{Q t} / N_{P}\right) \times 100\end{array}$ \\
\hline SSAC & 7.32 & 0.31 & 0.32 & 0.63 & 7.38 & 8.61 \\
SRAC & 6.81 & 0.35 & 0.24 & 0.59 & 9.75 & 8.67 \\
RSAC & 7.45 & 0.26 & 0.36 & 0.62 & 10.59 & 8.30 \\
SPAC & 5.98 & 0.40 & 0.18 & 0.60 & 7.20 & 10.00 \\
PSAC & 5.62 & 0.16 & 0.38 & 0.54 & 9.34 & 9.60 \\
\hline
\end{tabular}




\subsubsection{Mixing Efficiency}

To compare the mixing efficiency of the investigated coaxial mixer systems, the energy consumption, (P.t $\left.t_{m}\right)$ (Mishra and Joshi, 1994) of the different coaxial configurations were calculated. Figure (5.5-14) shows the energy consumption for five coaxial mixers utilized in this study at the speed ratio of $R_{n}=8$ and $R e=173$ in the co-rotating mode. These results demonstrate that the SPAC mixer was the most efficient coaxial configuration among the various investigated mixers in this study.

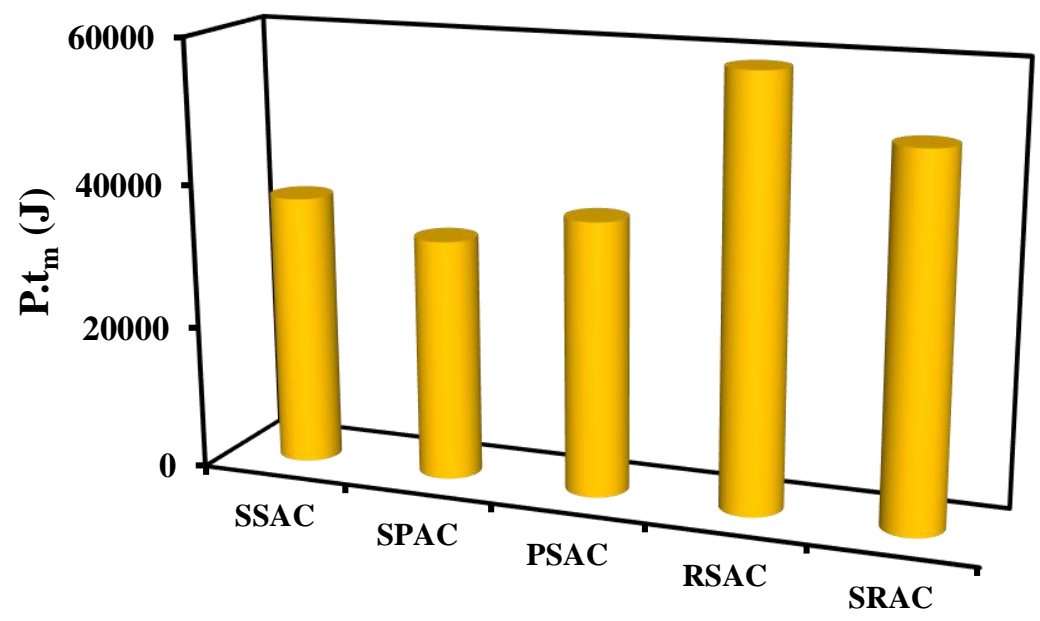

Coaxial mixer

Figure (5.5-14). Energy consumption for five coaxial mixers at $R_{n}=8\left(N_{c}=240 \mathrm{rpm}\right.$ and $\left.N_{a}=30 \mathrm{rpm}\right), 1.5 \%$ xanthan gum solution, and the co-rotating mode.

\subsubsection{Conclusion}

The hydrodynamic performances of the Scaba-Scaba-anchor coaxial (SSAC), ScabaRushton-anchor coaxial (SRAC), Rushton-Scaba-anchor coaxial (RSAC), Scaba-pitched blade-anchor coaxial (SPAC), and pitched blade-Scaba-anchor coaxial (PSAC) in the agitation the biopolymer xanthan gum solution, a yield-pseudoplastic fluid, were fully investigated. The experiments and simulations were conducted in the laminar-transitional 
regime and the co-rotating mode. Computational fluid dynamics (CFD) and electrical resistance tomography (ERT) techniques were utilized to assess the efficiency of these five coaxial mixers numerically and experimentally. A new correlation was developed for the coaxial mixers composed of an anchor and two different central impellers by incorporating the average Metzner-Otto constants $\left(K_{S}\right)$ of two central impellers into the Reynolds number. The results of the numerical simulations and experimental measurements showed that the lowest mixing time was attained by the SPAC mixer compared to the other coaxial mixing configurations. Further analysis revealed that the SPAC mixer generated the maximum velocity components at different locations throughout the tank. Furthermore, it was found that the highest shear rate with a maximum magnitude between the double central impellers and the anchor impeller was achieved by the SPAC configuration. To characterize these five coaxial mixers, the flow number and pumping effectiveness were also computed and the results demonstrated that the SPAC mixer was more effective than the others. Finally, the calculated mixing energy showed that the SPAC mixer was the most efficient coaxial configuration among the various investigated mixers in this study for the agitation of the yield-pseudoplastic fluids. 


\section{Chapter 6}

\section{Overall Conclusion}

The mixing performance of a novel coaxial system consisting of two high-speed central impellers and one anchor in the agitation of non-Newtonian yield- pseudoplastic fluids such as xanthan gum solution was investigated. The effects of important parameters such as the type of the central impellers, impeller spacing, speed ratio, and rheology of fluid on the hydrodynamic performance of this novel mixer were assessed.

Electrical resistance tomography (ERT) was used to measure the degree of homogeneity and to visualize the flow inside the mixing tank. The computational fluid dynamics (CFD) was employed to simulate the flow domain of the fluid and the design of experiments (DOE) combined with the response surface methodology (RSM) were utilized to analyze the mixing data and to determine the optimal design parameters. Using these techniques, various important features of the mixing of yield-pseudoplastic fluids with this novel coaxial mixers were analyzed as follows: 
- In the first stage of this project, for the first time, the effect of the rheological properties (consistency index $(K)$, power-law-index $(n)$, yield stress $\left(\tau_{y}\right)$ of the Herschel-Bulkley fluids on the mixing performance of a coaxial mixing system (Scaba-anchor) was investigated in terms of the mixing time and power consumption at five speed ratios for the co-rotating mode. The results of both numerical and experimental approaches demonstrated that the power drawn and mixing time of the Scaba-anchor coaxial mixer increased when the consistency index and yield stress were raised. However, the effect of power-law index on the mixing time and power consumption was not significant.

- The results of the design of experiments (DOE) and response surface methodology (RSM) showed that the interaction between the consistency index and yield stress was the most important interaction.

- The RSM analysis also revealed that the consistency index and speed ratio had the major influence on the mixing efficiency of the coaxial mixing system.

- It was found that mixing times computed through validated CFD model were correlated well with specific power consumption and Reynolds number for the coaxial system.

- For the first time, the hydrodynamic performance of the DSAC coaxial mixer (two Scaba impellers in combination with an anchor impeller) was assessed and compared to that of the SSAC coaxial mixer (single Scabe impeller in combination with an anchor impeller) with regards to the power consumption, mixing time, generated flow domain, and mixing efficiency. 
- It was found that the power curves generated by Pakzad et al. model (2013b) at different speed ratios collapsed to one curve in the co-rotating mode. In contrast, the power plots generated using Bao et al. model (2011) collapsed to one curve at the higher speed ratios while the power curves did not coincide reasonably at the lower speed ratios.

- The simulated flow domain through the validated CFD model revealed that the use of the single Scaba-anchor coaxial mixer (SSAC) resulted in the formation of a small cavern, well-mixed region, with the higher fluid velocities around the Scaba impeller while a large dead zone was formed in the upper part of the vessel and near the fluid surface.

- The results showed that when the double Scaba-anchor coaxial mixer (DSAC) was employed with the same power consumption as the single Scaba-anchor coaxial mixer (SSAC), fluid flow was intensified in both axial and radial directions leading to the elimination of the dead regions in the upper part of the mixing vessel. It was found that the use of two central impellers resulted in a more uniform distribution of the shear strain rate throughout the stirred vessel.

- To quantify the efficiencies of the DSAC and SSAC coaxial mixers, the mixing energy versus the mixing time Reynolds number at the same power consumption was plotted for both coaxial mixers. These results indicated that the DSAC coaxial stirrer was more effective than the SSAC stirrer in the mixing of the yieldpseudoplastic fluids.

- For first the time, the performances of the double Scaba-anchor coaxial (DSAC), double Rushton-anchor coaxial (DRAC), and double pitched blade-anchor coaxial 
(DPAC) mixers in the agitation of yield pseudoplastic fluids in the laminartransitional regime in the co-rotating mode were analyzed and compared.

- The collected ERT and CFD data were utilized to evaluate the hydrodynamic performances of these three coaxial mixers with respect to the mixing time, power consumption, generated fluid flow domain, and mixing energy.

- RSM plots demonstrated that the DSAC mixer had the lowest mixing time among the three coaxial mixers within the experimental range studied in this study.

- An analysis of the normalized mixing time curves for three coaxial mixers showed that the DSAC and DRAC mixing systems provided a better and faster tracer distribution. This was due to the formation of circulation loops, which eliminated the formation of the segregated zones inside the tank when these two coaxial impellers were utilized. However, these mixing curves for the DPAC mixer showed two plateau sections at two monitoring points. The presence of these plateau sections was due to the axial flow compartmentalization, which resulted in the formation of the segregated zones inside the agitated tank.

- The simulated flow domain by the CFD model proved that both DSAC and DRAC mixers generated similar stable parallel flow patterns at the impeller spacing equal to $T / 2$ while the flow pattern created by the DPAC mixer was unstable resulting in less efficient mixing operation.

- The analysis of the velocity profiles revealed that the DSAC mixer created a more uniform velocity distribution throughout the tank compared to the other two coaxial mixers. 
- it was found that the DRAC mixer induced the highest shear rate with a maximum magnitude close to the upper and lower central impellers. However, the shear rate profile generated by the DSAC mixer in the radial direction was more consistent than those by the other two mixers.

- The calculated flow numbers for these coaxial mixers showed that the DSAC mixer was more effective with respect to the pumping rate at the operating conditions studied in this research work.

- $\quad$ The mixing energy versus the speed ratio was plotted for three coaxial mixers and these data revealed that the DSAC mixer was more efficient for the agitation of non-Newtonian yield-pseudoplastic fluids than the other two coaxial mixers.

- For the first time through this study, the influence of the impeller spacing on the performance of a coaxial mixer composed of two central impellers and an anchor in the agitation of highly viscous non-Newtonian fluids was investigated.

- Power number, flow number, mixing time, and pumping effectiveness produced by CFD and ERT were employed to assess the performances of the coaxial mixer as a function of the spacing between two central impellers for both co-rotating and counter-rotating modes. It was found that the mixing in co-rotating mode was more efficient than the counter-rotating mode.

- In order to obtain the optimal impeller spacing, the energy consumption, which is the product of the power consumption and mixing time, was calculated for various impeller spacing. It was found that the coaxial mixer with the impeller spacing of $0.175 \mathrm{~m}$ and the impeller clearance of $0.185 \mathrm{~m}$ was the most efficient 
system with the minimum energy consumption among the investigated configurations.

- The velocity vector plot, velocity contour plot, streamlines plot, axial velocity profile, radial velocity profile, tangential velocity profile, shear rate profile, and apparent viscosity profile were calculated through the validated CFD model to analyze the characteristic flow pattern generated by the double Scaba-anchor coaxial mixer as a function of the spacing between two central Scaba impellers.

- The results demonstrated that the changes in the impeller spacing for the coaxial mixer resulted in two stable flow patterns in the mixing of yield-psudoplastic fluid: parallel flow and merging flow.

- For the first time the hydrodynamic performances of the Scaba-Scaba-anchor coaxial (SSAC), Scaba-Rushton-anchor coaxial (SRAC), Rushton-Scaba-anchor coaxial (RSAC), Scaba-pitched blade-anchor coaxial (SPAC), and pitched bladeScaba-anchor coaxial (PSAC) in the agitation the biopolymer xanthan gum solution, a yield-pseudoplastic fluid, were fully investigated.

- A new correlation was developed for the coaxial mixers composed of an anchor and two different central impellers by incorporating the average Metzner-Otto constants $\left(K_{s}\right)$ of two central impellers into the Reynolds number.

- The results of the numerical simulations and experimental measurements showed that the lowest mixing time was attained by the SPAC mixer compared to the other coaxial mixing configurations. 
- Further analysis revealed that the SPAC mixer generated the maximum velocity components at different locations throughout the tank. Furthermore, it was found that the highest shear rate with a maximum magnitude between the double central impellers and the anchor impeller was achieved by the SPAC configuration.

- To characterize these five coaxial mixers, the flow number and pumping effectiveness were also computed and the results demonstrated that the SPAC mixer was more effective than the others.

- Finally, the calculated mixing energy showed that the SPAC mixer was the most efficient coaxial configuration among the various investigated mixers in this study for the agitation of the yield-pseudoplastic fluids.

\subsection{Recommendation for Future}

The experimental and numerical results of this study drew attention to the following areas for future considerations:

- The effects of the other types of the central impellers and the position of the central impeller on the performances of the coaxial mixers should be assessed.

- The performance of the coaxial mixers composed of double central impellers (identical/different) for the multiphase mixing operations (e.g. gas-liquid, solidliquid, and emulsion) should be assessed.

- The efficiency of the coaxial mixers composed of double central impellers (identical/different) should be analyzed for the other types of non-Newtonian fluids. 
- The scale-up criteria for the coaxial mixing systems should be developed.

- The performance of the coaxial mixers in the continuous mode should be analyzed. 


\section{Nomenclature}

$a$

$b$

$c$

$d$

A

$C_{a}$

$C_{1}$

$\mathrm{C}_{2}$

$C_{3}$

$D_{c}$

$D a$

$D_{m}$

$D_{C}$

Ds

$e_{i}$

$f_{p(a)}$

F

$\vec{F}$

$g$

H

$H_{a}$

K

$k$

$K_{p}$

$K_{R N}$

$K_{S}$

$K_{s(s)}$

$K_{t r}$ constant, dimensionless

constant, dimensionless

constant, dimensionless

constant, dimensionless

surface area, $m^{2}$

anchor impeller gap between the anchor and the wall and tank base, $m$

impeller submergence, $m$

impeller spacing, $m$

impeller off-bottom clearance, $m$

central impeller diameter, $m$

anchor impeller diameter, $m$

molecular diffusivity, $m^{2} s^{-1}$

central impeller diameter, $m$

Scaba impeller diameter, $m$

error

anchor power fraction

force (drag force), $N$

external (body) force, $N$

gravitational acceleration, $m s^{-2}$

height of fluid in vessel, $m$

anchor impeller blade height, $m$

consistency index, $P a s^{n}$

number of factor

proportionality constant of the power number, dimensionless

speed ratio constant, dimensionless

Metzener-Otto constant for calculating shear rate, dimensionless

Metzener-Otto constant for calculating shear rate for Scaba impeller, dimensionless

shift factor 


\begin{tabular}{|c|c|}
\hline$m$ 。 & initial mass fraction \\
\hline$m_{\infty}$ & final mass fraction \\
\hline$m(t)$ & mass fraction at any time \\
\hline$M(t)$ & degree of homogeneity \\
\hline$M$ & corrected torque, N.m \\
\hline$M_{m}$ & measured torque, $N . m$ \\
\hline$M_{r}$ & residual torque, $N . m$ \\
\hline$n$ & power-law index, dimensionless \\
\hline$n-1$ & Exponent constant, dimensionless \\
\hline$N$ & impeller rotational speed, rot $s^{-1}$ \\
\hline$N^{\prime}$ & characteristic mixing speed, rot $s^{-1}$ \\
\hline$N_{a}$ & anchor impeller speed, rot $s^{-1}$ \\
\hline$N_{C}$ & central impeller rotational speed, rot $s^{-1}$ \\
\hline$N_{c o}$ & coaxial rotational speed, rot $^{-1}$ \\
\hline$N_{Q}$ & impeller flow number, dimensionless \\
\hline$N_{Q L}$ & lower impeller flow number, dimensionless \\
\hline$N_{Q U}$ & upper impeller flow number, dimensionless \\
\hline$N_{p}$ & power number, dimensionless \\
\hline$N_{P U}$ & power number upper impeller, dimensionless \\
\hline$N_{P L}$ & power number lower impeller, dimensionless \\
\hline$P$ & power, $W$ \\
\hline$p$ & pressure, $P a$ \\
\hline$P_{t o t}$ & total power (anchor \& Scaba), $W$ \\
\hline$Q$ & pumping capacity, $m^{3} s^{-1}$ \\
\hline$R_{i}$ & impeller radius, $m$ \\
\hline$R^{2}$ & correlation coefficient, dimensionless \\
\hline$R_{n}$ & speed ratio $\left(N_{C} / N_{a}\right)$, dimensionless \\
\hline$S_{\varphi}$ & generalized source term, variable unit \\
\hline$t$ & time, $s$ \\
\hline$t_{a}$ & anchor impeller thickness, $m$ \\
\hline$t_{m}$ & mixing time, $\mathrm{s}$ \\
\hline
\end{tabular}




$\begin{array}{ll}t r & \text { tip speed ratio }\left(D_{C} N_{C} / D_{a} N_{a}\right), \text { dimensionless } \\ T & \text { tank diameter, } m \\ T & \text { temperature, }{ }^{\circ} C \\ V_{a} & \text { axial velocity, } m s^{-1} \\ V_{r} & \text { radial velocity, } m s^{-1} \\ V_{t} & \text { tangential velocity, } m s^{-1} \\ V_{m a x}^{*} & \text { maximum magnitude of velocity, } m s^{-1} \\ V_{t i p} & \text { impeller tip speed, } m s^{-1} \\ \vec{v} & \text { mean velocity, } m s^{-1} \\ V & \text { fluid volume, } m^{3} \\ w & \text { local mass fraction, dimensionless } \\ w_{a} & \text { anchor impeller blade width, } m \\ X_{i}, X_{j} & \text { independent variables } \\ Y & \text { response function } \\ Y_{1} & \text { predicted response mixing time function, } S \\ Y_{2} & \text { constant, dimensionless } \\ \pi & \text { constant, dimensionless } \\ \pi_{1} & \text { constant, dimensionless } \\ \pi_{2} & \\ \pi_{2} & \text { constant, dimensionless } \\ & \end{array}$

\section{Greek letters}

$\begin{array}{ll}\gamma^{\prime} & \text { shear rate, } s^{-1} \\ \gamma_{a v}^{\prime} & \text { average shear rate in the mixing vessel, } s^{-1} \\ \beta_{0} & \text { constant, dimensionless } \\ \beta_{i} & \text { constant, dimensionless } \\ \beta_{i i} & \text { constant, dimensionless } \\ \beta_{i j} & \text { constant, dimensionless } \\ \eta & \text { non-Newtonian viscosity, Pa.s }\end{array}$




$\begin{array}{ll}\eta_{E} & \text { pumping effectiveness, dimensionless } \\ \mu & \text { Newtonian viscosity, } P a . s \\ \mu_{e f f} & \text { effective viscosity, } P a . s \\ \mu_{0} & \text { zero shear viscosity, } P a . s \\ \mu_{\text {app }} & \text { apparent viscosity, } P a . s \\ \rho & \text { fluid density, } K g . m^{-3} \\ \tau & \text { shear stress, } P a \\ = & \text { viscous stress tensor, } P a \\ \tau & \text { yield stress, } P a \\ \tau_{y} & \text { kinematic viscosity of the liquid, } m^{2} s^{-2} \\ v & \text { constant, dimensionless } \\ \pi & \text { constant, dimensionless } \\ \pi_{2} & \text { constant, dimensionless } \\ \pi_{3} & \text { angular velocity, } s^{-1} \\ \omega & \end{array}$

\section{Subscripts}

app apparent

$g \quad$ generalized

tr tip speed ratio

tip tip speed impeller

\section{Dimensionless Numbers}

Re Reynolds number, dimensionless

$R_{g} \quad$ generalized Reynolds number, dimensionless

$R e_{m} \quad$ Reynolds mixing time, dimensionless

\section{Abbreviations}

$A C \quad$ alternating current, ampere (amp) or $A$

CFD computational fluid dynamics 


\begin{tabular}{|c|c|}
\hline$C P U$ & central processing unit \\
\hline$D A S$ & data acquisition system \\
\hline$D O E$ & design of experiments \\
\hline$D S A C$ & double Scaba-anchor coaxial \\
\hline$D P A C$ & double pitched-blade -anchor coaxial \\
\hline$D R A C$ & double Rushton -anchor coaxial \\
\hline$E R T$ & electrical resistance tomography \\
\hline$h p$ & horse power \\
\hline$H P C V L$ & high performance computational virtual laboratory \\
\hline$L B P$ & linear back projection \\
\hline$L D A$ & laser doppler anemometry \\
\hline$L D V$ & laser doppler velocimetry \\
\hline$M R F$ & multiple reference frame \\
\hline$P B A$ & Pitched blade anchor \\
\hline$P H$ & potential of hydrogen \\
\hline$P I V$ & particle image velocimetry \\
\hline PLIF & planner laser induced fluorescence \\
\hline$P S A C$ & Pitched-blade-Scaba-anchor coaxial \\
\hline$R A M$ & random access memory \\
\hline$R S M$ & response surface methodology \\
\hline$R S A C$ & Rushton-Scaba-anchor coaxial \\
\hline$S C G$ & sensitivity conjugate gradient \\
\hline SIMPLE & semi-Implicit Method for Pressure-Linked Equation \\
\hline SIMPLEC & SIMPLE consistent \\
\hline SSAC & single Scaba- anchor \\
\hline SPAC & Scaba-pitched-blade turbine-anchor coaxial \\
\hline SRAC & Scaba-Rushton turbine-anchor coaxial \\
\hline SSAC & Scaba-Scaba anchor coaxial \\
\hline$U K$ & United Kingdom \\
\hline
\end{tabular}




$\begin{array}{ll}\text { USA } & \text { United State of America } \\ U V & \text { ultraviolet } \\ \text { Operations } & \\ \frac{\partial}{\partial t} & \text { partial derivative with respect to time } \\ \frac{D}{D t} & \text { substantive derivative } \\ \nabla & \text { del or nabla operator } \\ \Delta & \text { difference operator } \\ \Sigma & \text { summation operator }\end{array}$




\section{Bibliography}

$\underline{\mathbf{A}}$

Alverz, M.M., Arriata, P.E., Muzzio, F.J, 2002. Laminar mixing I eccentric stirred tank systems. Can. J. Chem. Eng. 80, 546-557.

Amanullah, A., Hjorth, S.A, Nienow, AW. 1997. Cavern sizes generated in highly shear thiningviscous fluids by Scaba 3SHPI impeller. Food bioprod. Process, 75, 232-238.

ANSYS $®$ Academic Research, Release 15, Help System, Coupled Field Analysis Guide, ANSYS, Inc.

Armenante, P. A., Mazzarotta, B., Chang, G., 1999. A power consumption in stirred tanks provided with multiple Pitched-Blade turbines. Ind. Eng. Chem. Res., 38, 28092816

Ascanio, G., Foucault, S., Tanguy, P. A., 2003 Performance of a new mixed down pumping impeller. Chem. Eng. Technol. 26, 908-911.

$\underline{\mathbf{B}}$

Bao, Y., Bo, Y., GAO, Z., Zhang, Z., Liu, T., GAO, X., 2011. Power demand and mixing performance of co axial mixers in non-Newtonian fluids. J. Chem. Eng. Jpn.44, 57-66.

Barber, C. D., Brown, B.h., 1984. Applied potential tomography. J. of Phys. E: Sci. Instrum 17, 723-733.

Bates, R.L., Fondy, P.L., R.R. 1963. An examination of some geometric parameters of impeller power. Ind. Eng. Chem. Process. Des. Dev, 2, 310-314.

Bates, R. L., Fondy and Fenic, J.G., 1966 “in mixing - Theory and practice "(ed. V. Uh 1 and J.B.Gray), Vol. 1, P. 143. Academic press, New York.

Bertrand, F., Tanguy, P. A., Britto-De La Fuente, E., 1996. A new perspective for the mixing of yield stress fluids with anchor impellers. J. Chem. Eng. Jpn. 29, 51-58.

Bird, R.B., Stewart, W.E., Lightfoot, E.N., 2007. Transport Pehenomena. Erd ed., John Wiley\&Sons. Inc., New York. 
Bouaifi, M., 1997M. Bouaifi, M. Roustan, R. Djebbar, 1997. Hydrodynamics of multistage agitated gas-liquid reactors Récents Progrès en Genie des Procédés Vol 11Lavoisier, Paris, no. 52, 137-144.

Bouaifi, M., Roustan, M., 2001. Power consumption. Mixing time and homogenization energy in dual-impeller agitated gas-liquid reactors. Chem. Eng. Proc. 40, 87-95.

Box, G.E.P. and Wilson, K.B. 1951. "On the experimental attainment of optimum conditions", J. Roy. Statist. Soc., B13,

Bonnot, S., F. Cabaret, F., Fradette, P.A. Tanguy, P. A., 2007. Characterization of mixing patterns in a coaxial mixer. Chem. Eng. Re. De. 85, 1129-1135.

Bujalski, W., Jaworski, Z., Nienow, A.W., 2002. CFD study of homogenization with dual ryshton turbine-comparison with experimental results part II: the multiple reference frame. Chem. Eng. Res. Des., 80, 97-104.

Buwa V., Dewan A., Nasser A.F., Durst F., 2006. Fluid dynamics and mixing of singlephase flow in stirred vessel with a grid disc impeller: experimental and numerical investigations, Chem. Eng. Sci., 61, 2815-2822.

$\underline{\mathbf{C}}$

Calderbank, P.H., Moo-Young, M.B., 1961. The power characteristics of agitators for the mixing of Newtonian and non-Newtonian fluids. Trans Inst. Chem. Eng. 39, 337-347.

Chhabara, R.P., Richardson, J.F. 1999. Non-Newtonian Flow in Process Industries. $1^{\text {st }}$ ed. Butterworth Heinemann, Oxford.

Cronin, D.G., Nienow, A.W., Moody, G.W., 1994. An experimental study of mixing in a proto-fermenter agitated by dual Rushton turbines Trans IChemE, Pt C, Foods Bioprod Proc, 72, 35-40. 


\section{$\underline{\mathbf{D}}$}

Delaplace, G., Thakur, R.K., Bouvier, L., Andre, C., Torrez, C., 2007. Dimentional analysis for planetary mixer: mixing time and Reynolds number. Chem Eng. Sci. 62, 1442-1447.

Distelhoff, M.F.W., Marquis, A.J., Nouri, J.M., Whitelaw, J. H., 1997. Scalar mixing measurements in batch operated stirred tanks. Chan. J. Chem. Eng. 75, 641-652.

Dubois, C., Thibault, F., Tanguy, P.A., Ait-Kadi, A., 1996. Characterization of viscus mixing in twin intermeshing conical helical mixers. I. Chem. E. Symp. 140, 972-977.

$\underline{\mathbf{E}}$

Eklund, D. E. and Teirfolk, 1981. J. E. TAPPI J. 64, 63.

Espinosa-Solares, T., Brito De La Fuente, E., Tencante, A., Tanguy P.A., 1997. Power consumption of dual turbine-helical impeller mixers in ungassed conditions. Chem. Eng. J. 67, 215-219.

$\underline{\mathbf{F}}$

Farhat, M., Fradette, L., Tanguy, P.A., 2008. Revisiting the performance of a coaxial mixer. Ind. Eng. Chem. Res. 47, 3562-3567.

Farhat, M., Rivera, C., Fradette, L., Heniche, M., Tanguy, P.A., 2007. Numerical and experimental study of dual-shaft coaxial mixer with vscus fluids. Ind. Eng. Chem. Res. 46, 5021-5031.

Ford, C., Ein-Mozaffari, F., Bennington, C.P.J., Taghip0ur, F., 2006. Simulation of mixing dynamics in agitated pulp stuck chests using CFD. AIChE J. 52, 3562-3569.

Fort, I., 1986. Flow and turbulence in vessels with axial impellers, in mixing III (Editted by V. W. UhI and J.B. Gray). Academic Press, New York.

Fordham'E. J., Hall' L. D., Ramakrishnan, T. S., Sharpe, M. R., and C. Hall, c., 2004. Saturation gradients in drainage of porous media: NMR imaging measurements. AIChE J. 39, 1431-1443. 
Foucault, S., Acanio, G., Tanguy, P.A., 2004. Coaxial mixer hydrodynamics with Newtonian and non-Newtonian fluids. Chem. Eng. Technol. 27, 324-329.

Foucault, S., Acanio, G., Tanguy, P.A., 2005. Power characteristics in coaxial mixing: Newtonian and non-Newtonian fluids. Ind. Eng. Chem.Res. 44, 5036-5043.

Foucault, S., Acanio, G., Tanguy, P.A., 2006. Mixing time in coaxial mixers with Newtonian and non-Newtonian fluids. Ind. Eng. Chem. Res. 45, 352-359.

\section{$\underline{\mathbf{H}}$}

Herschel, W.E., Bulkley, R., 1926. Measurments of consistency as applied to rubberbenzene solutions. Proc. Am. Soc.Test. Master. 26, 621-633.

Holden, P.J., Wang, M., Mann, R., Dickin, F.J., Edwards, R.B., 1998. Imaging stirredvessel macromixing using electrical resistance tomography. AIChE J. 44, 780-790.

Hiraoka, S., Kato, Y., Tada, Y., Ozaki, N., Murakami, Y., Lee, Y.S., 2001. Power consumption and mixing time in an agitated vessel with double impellers Proceedings of 4th International Symposium on Mixing in Industrial Processes (on CD-ROM), PROGEP, Toulouse, 92-97.

Huang, S. M., Xie, C. G., Thorn, R., snowden, D., Beck, M.S., 1992. Design of sensor electronics for electrical capacitance tomography, IEE Proc. G1 139, 83-88.

Hudcova, V., Machon, V., A.W. Nienow, A.W., 1989. Gas-liquid dispersion with dual Rushton turbine impellers' Biotechnol Bioeng, 34, 617-628.

\section{$\underline{\mathbf{I}}$}

Iranshahi, A., Heniche, M., Bertrand, F., Tanguy, P.A., 2006. Numerical investigation of the mixing efficiency of ekato paravisc impeller. Chem. Eng. Sci 61, 2609-2617.

$\underline{\mathbf{J}}$

Jaworski, Z., Bujalski, W., Otomo, N., A.W. Nienow, A.W., 2000. CFD study of homogenization with dual Rushton turbines-comparison with experimental results. Part I: initial studies Trans IChemE, Pt A, Chem Eng Res Des, 78, 327-333. 
$\underline{\mathbf{K}}$

Kazemzadeh, A., Ein-Mozaffari, F., Lohi, A., Pakzad, L., 2016a. Effect of the rheological properties on the mixing of Herschel-Bulkley fluids with the coaxial mixers: applications of tomography, CFD, and response Surface methodology. Can. J. Chem. Eng. (accepted).

Kazemzadeh, A., Ein-Mozaffari, F., Lohi, A., Pakzad, L., 2016b. Investigation of hydrodynamic performances of coaxial Mixers in agitation of yield-pseudoplasitc fluids: single and double central impellers in combination with the Anchor. Chem. Eng. J., 294, 417-430.

Kazemzadeh, A., Ein-Mozaffari, F., Lohi, A., Pakzad, L., 2016c. A new perspective in the evaluation of the mixing of biopolymer solutions with different coaxial mixers comprising of two dispersing impellers and a wall scraping anchor. Chem. Eng. Des. Res. J. (accepted).

Kazemzadeh, A., Ein-Mozaffari, F., Lohi, A., Pakzad, L., 2016d. Flow patterns in agitation of non-Newtonian yield-pseudoplastic fluids generated by the coaxial mixer composed of double Scaba and an anchor. Chem. Eng. Commun. (Submitted).

Kazemzadeh, A., Ein-Mozaffari, F., Lohi, A., Pakzad, L., 2016e, Mixing of nonNewtonian biopolymer solutions with the coaxial Mixers composed of two different central impellers and an anchor (submitted).

Kaminoyama, M., Akabane, K., Arai, K., Saito, F., Kamiwano, M., 1994. Numerical analysis of flow of a Bingham fluid in an anchor impeller. Int. Chem. Eng. 34, 263-269.

Kaminoyama, M., Saito, F., Kamiwano, M., 1990. Flow analogy of pseudo-plastic liquid in geometrically similar stirred vessels based on numerical analysis. J. Chem. Eng. Jpn., 23, 214-221.

Kaminoyama. M., Taguchi, S., Misumi, R., Nishi, K., 2005. Monitoring stability of reaction and dispersion states in a suspension polymerization reactor using electrical resistance tomography measurements. Chem. Eng. Sci. 60, 55131-2152. 
Khopkar, A. R., Fradette, L., Tanguy, P.A., 2007. Hydrodynamics of a dual shaft mixer with Newtonian and non-Newtonian. Chem. Eng. Res, Des. 85, 867-871.

Kim, S., Nkaya, A.N., Dyakowski, T., 2006. Measurment of mixing of two miscible liquids in a stirred vessel with electrical resistance tomography. Int. Commun. In Heat and Mass transfer 33, 1088-1095.

Kohler, S., Hemmerle, W., 2003. Analysis of the Power characteristic of coaxial agitator with varied diameter and speed ratio of inner and outer mixing device. Proc. $11^{\text {th }}$ Eur. Conf. Mixing, Bamberg, Geometry, 14-17.

Komori, S. Murakami, Y., 1988.Turbulent mixing in baffled stirred tanks with verticalblade impellers AIChE J, 34, 932-937.

Kuriyama, M., Inomata, H., Arai, K., Saito. 1982. Numerical solutions for the flow of highly viscous fluid in agitated vessel with anchor impeller. AIChE J. 28, 385-391.

Kuboi, R., Nienow, A.W., Allsford, K. 1983. Multipurpose stirred tank facility for flow visulisation and dual impeller power measuirement. Chem. Eng. Com., 22, 29-39.

$\underline{\mathbf{L}}$

Letellier B., Xuereb C., Swaels P., Hobbes P., 2002. Scale-up in laminar and transient regims of multi-stage stirrer, a CFD approach, Chem. Eng. Sci., 57, 4617-4632.

Lucas, G.P., Cory, J., Mann, R., Dickin, F.J., Edwards, R.B., 1999. Measurment of solids volume fraction and velocity distributions in solid-liquid flows using dual-plane electrical resistance tomography. Flow Meas. Instrum. 10, 249-258.

Luo, J.Y., Gosman, A.D., Issa, R.I., 1994. Prediction of impeller induced flows in mixing vessels using multiple frames of reference. Ins. Chem. Eng. Symp. 136, 549-556.

Luo, J.Y., Gosman, A.D., Issa, R.I., Middleton, J.C., Fitzgerald, M.K., 1993. Full flow field computation of mixing in baffled stirred vessels. Trans IChem E., 71, 342-344. 


\section{$\underline{\mathbf{M}}$}

Mann R., Dickin F. J., Wang M., Dyakowski T., Williams R. A., Edwards R. B., Forrest A. E., and Holden P. J. 1997. Application of electrical resistance tomography to interrogate mixing process at plant scale. Chem. Eng. Sci., 52, 2087-2097.

Madupu, A., Mazumdar, Jinciples, Z., Roelant, D, D. and Srivastava, R. 2005. Electrical resistance tomography for real-time mapping of the solid-liquid interface in tanks containing optically opaque fluids. Proc. SPIE - Int. Soc. Opt. Eng., 5674, 36-46.

Mahamoudi, S.M.S., Manneskis, M., 1991. The variation of flow pattern and mixing time with impeller spacing in stirred vessels with two Rushton impellers M. Bruxelmane, G. Froment (Eds.), Proceedings of 7th European Conference on Mixing, K-VIV, Antwerp, $17-24$.

Magelli, F., Fajner, D., Pasquali, G., Nocentini M., 1986. Fluid-dynamic behaviour and mixing times of multiple impeller stirred tanks with low viscosity liquids Proceedings of 5th Yugoslavian-Austrian-Italian Chemical Engineering Conference, Kardelj University, Ljubljana, 323-330.

Mishra, V., Joshi, J., 1994. Flow generated by disc turbine. IV: Multiplimpellers. Chem. Eng. Res. Des., 72, 657-668.

Mukataka, S., Kataoka, H., Takahashi, J., 1981. Circulation time and degeree of fluid exchange between upper and lower circulation regions in a stirred vessel with a dual impeller J Ferment Technol, 59, 303-307.

Montante G., M. Mostek, M., M. Jahoda, M., F. Magelli, F., 2004. CFD simulations and experimental validation of homogenisation curves and mixing time in stirred Newtonian and pseudoplastic liquids Chem Eng Sic.

Myers, R.H. and Montgomery, D.C. 2002. Response Surface Methodology: Process and Product Optimization Using Designed Experiments (2nd ed.), John Wiley \& Sons, Inc.

Mavros P., Mann R., Vlaev S. D., and Bertrand J. 2001. "Experimental Visualization and CFD Simulation of Flow Patterns Induced by a Novel Energy- Saving Dualconfiguration Impeller in Stirred Vessels.” Trans IChem. E., 79 (part-A) 857-866. 
Metzner, A.B., Otto, R.E., 1957. Agitation of non-Newtonian fluids. AIChE J. 3, 3-11.

Moo-Young, M., Tichar, K., Dullib, A.L. 1972. The blending efficiencies of some impellers in batch mixing. AIChE j. 18, 178-182.

Murthy, S.S., Jayanti, S., 2003b. Mixing of power-law fluids using anchors: MetznerOtto concept revisited. AIChE J. 49, 30-40.

Murthy, S.S., Jayanti, S., 2003a. Mixing of pseudoplastic fluids using helical ribbon impellers, AIChE J. 49, 2768-2772.

Novak, V. and Riger F., 1973. Colln. Czech. Chem. Commun. 38, 350.

$\underline{\mathbf{O}}$

Ohta, M., Kuriyama, M., Arai, K., Saito, S., 1985. A two-dimentional model for the secondary flow in agitated vessel with anchor impeller. J. Chem. Eng. Jpn. 18, 81-84.

$\underline{\mathbf{P}}$

Pakzad, L., Ein-Mozaffari, F., Chan, P., 2008a. Using computational fluid dynamics modeling to study the mixing of pseudoplastic fluids with a Scaba 6SRGT impeller. Chem. Eng. Proc. 47, 2218-2227.

Pakzad, L., Ein-Mozaffari, F., Chan, P., 2008b. Using electrical resistance tomography and computational fluid dynamics modeling to study the formation of cavern in the mixing of pseudoplastic fluid possessing yield stress. Chem. Eng. Sci. 63, 2508-2522.

Pakzad, L., Ein-Mozaffari, F., Chan, P., 2008c. Measuring mixing time in the agitation of non-Newtonian fluids through elrctrical resistance tomography. Chem. Eng. Technol. 12, 1838-1845.

Pakzad, L., Ein-Mozaffari, F., Upreti, S.R., Lohi, A., 2013a. Characterization of the mixing of non-Newtonian fluids with a Scaba 6SRGTimpeller through ERT and CFD. Can. J. Chem. Eng. 91(1) 90-100.

Pakzad, L., Ein-Mozaffari, F., Upreti, S.R., Lohi, Ali. 2013b. Agitation of HerschelBulkley fluids with the Scaba-anchor coaxial mixers, Chem. Eng. Res. Des. 91, 761-777. 
Pakzad, L., Ein-Mozaffari, F., Upreti, S.R., Lohi, A., 2013c. Evaluation of the mixing of non-Newtonian biopolymer solutions in the reactors equipped with coaxial mixers through tomography and CFD. Chem. Eng. J. 215-216, 279-296.

Pakzad, L., Ein-Mozaffari, F., Upreti, S.R., Lohi, A., 2013d. Experimental and numerical studies on mixing of yield-pseudoplastic fluids with a coaxial mixer. Chem. Eng. Commun. 200(12), 1553-1557.

Pant, H.J., Kundu, A., Nigam, K.D.P., 2001. Radiotracer Application in chemical process industry. Rev. Chem. Eng. 17, 165-252.

Patankar, S.V., 1980. Numerical Heat Transfer and Fluid flow. Taylor \& Francis Group, New York.

Pedrosa, S.M.C.P., Nunhez, J.R., 2000. The behavior of stirred vessels with anchor type impellers. Comput. Chem. Eng. 24, 1745-1751.

Prajapati, P., Ein-Mozaffari, F., 2009. CFD investigation of mixing of yieldpseudoplastic fluids with anchor impeller. Chem. Eng. Technol. 32, 1211-1218.

\section{$\underline{\mathbf{R}}$}

Ray, S., Lalman, J.A., Biswas, N., 2009. Using the Box-Benkhen technique to statistically model phenol photocatalytic degradation by titanium dioxide nanoparticles, Chem. Eng. J. 150, 15-24.

Ranade, V.V., 2002. Computational Flow Modeling for Chemical Reactor Engineering ACADEMIC Press, San Diego.

Ray, S., Lalman, J.A., Biswas, N., 2009. Using the Box-Benkhen technique to statiscally model phenol photocatalytic degradation by titanium dioxide nanoparticle, Chem. Eng. J. $150,15-24$.

Rao, M.A., 1999. Rheology of Fluid and Semisolid Foods Principles and Applications. Aspen, Inc., Maryland.

Rieger, F., Novak, V., 1973. Power consumption of agitators in highly viscous nonNewtonian liquids. Trans. Instn Chem. Engrs. 51, 105-111. 
Rewatkar, V.B., Joshi, J.B., 1991. Effect of impeller design on liquid phase mixing in mechanically agitated reactors. Chem. Eng. Commun. 91, 322-353.

Ricard, F., Brechtelsbaur, C., Xu, X.Y., Larence, C.J., 2005. Monitoring of multiphase pharmaceutical processes using electrical resistance tomography. Trans IChem E., 83, 794-805.

Rivera, C., Foucault, S., Heniche, M., Espinosa-Slares, T., Tanguy, P.A., 2006. Mixing analysis in a coaxial mixer, Chem. Eng. Sci. 61, 2895-2907.

Rubart, L., Bohme, G., 1991. Numerical simulation of shear-thinning flow problems in mixing vessels. Theoret. Comput. Fluid Dynamics 3, 95-115.

Rudolph, L., Atiemo-Obeng, V., Schaefer, M., Kraume, M., 2009. Power consumption and blend time of coaxial tank mixing systems in non-Newtonian fluids. In: proceedings of the $13^{\text {th }}$ European Conference Mixing, London, United Kingdom, 439-446.

Rudolph, L., Atiemo-Obeng, V., Schaefer, M., Kraume, M., 2007. Experimental and numerical analysis of power consumption for mixing of high viscosity fluids with coaxial mixer. Chem. Eng. Res. Des. 85, 568-572.

Ruszkowski, S., 1994. A rational method for measuring blending performance and comparison of different impeller types. Proc. $8^{\text {th }}$ Eur. Conf. Mixing, Cambridge UK, 283-292.

Rutherford, K., Lee K.V., Mahmoudi, S.M.S., Yianneskis M., 1996.Hydrodynamic characteristics of dual Rushton impeller stirred vessels AIChE J, 42 (1996), 332-346.

\section{$\underline{\mathbf{S}}$}

Sano, Y., Usui, H., 1985. Interrelation among mixing time, power number, and discharge flow rate number in baffled mixing vessels. J. Chem. Eng. Jpn. 18, 47-52.

Saverux, F., Jay, P., Albert, M., 2007. Viscoplastic fluid mixing in a rotating tank. Chem. Eng. Sci. 62, 2290-2301. 
Schneider, T., Todtenhaupt, E., 1990. Mixing times and heat transfer in coaxial stirrers, EKATO Ruehr-Mischtech. G.m.b.H., Schopfheim, Germany. Chem. Ing. Tech. 62, 208209.

Sestak, J., Zinty, R., Houska, M., 1986. Anchor-agitated systems: power input correlation for pseudo-plastic and thixotropic fluid in equilibrium. AIChE J. 32, 155-158.

Shiue, S.J., Wong. C.W., 1984. Studies on homogenization efficiency of various agitators in liquid blending. Can J. Chem. Eng. 62, 602-609.

Smith, J.M., Warmoeskerken, M.M.C.G., Zeef E., 1987. Flow conditions in vessels dispersing gases in liquids with multiple impellers C.S. Ho, J.Y. Oldshue (Eds.), Biotechnology Processes: Scale-up and Mixing, AIChE, New York, 107-115.

Solomon, J., Elson, T.P., Nienow, A.W., 1981. Cavern sizes in agitated fluids with a yield stress. Chem. Eng. Commun. 11, 143-164.

\section{$\underline{\mathbf{T}}$}

Tabor G., Gosman A. D., and Issa R. I. 1996. "Numerical Simulation of the Flow in a Mixing Vessel Stirred by a Rushton Turbine.” Inst. Chem. Eng. Symp. 140, 25-34.

Taguchi, H., T. Kimura T., 1970. Studies on geometric parameters in fermentor design. I. Effect of impeller spacing on power consumption and volumetric oxygen transfer coefficient J Ferment Technol, 48, 117-124

Tanguy, P.A., Bertrand, F., Brito-De La Fuente, E., 1994. Mixing of viscoplastic fluids with an anchor impellers. I. Chem. E. Symp. 136, 525-532.

Tanguy, P.A., Thibault, F., Brito-De La Fuente E., Espinosa. T., Tecante, A., 1997. Mixing performance induced by coaxial flat blade-helical ribbon impellers rotating at different speeds. Chem. Eng. Sci. 52, 1733-1741.

Tanguy, P.A., Thibault, F., Brito-De La Fuente E., 1996. A new investigation of the Metzner-Otto concept for anchor mixing impellers. Can. J. Chem. Eng. 74, 222-228.

Taterson, G.B., 1991. Fluid Mixing and Gas Dispersion in Agitated Tanks. McGraw-Hill, New York. 
Thibault, F., Tanguy, P.A., 2002. Power draw analysis of coaxial mixer with Newtonian and non-Newtonian fluids in the laminar regime. Chem. Eng. Sci 57, 3861-3872.

Triveni, B., Vishwanadham, b., Madhavi, T., Venkateshwar, S., 2009. Mixing studies of non-Newtonian fluids in an anchor agitated vessel. Chem. Eng. Res.Des. 88, 809-818.

Toye, D., Marchot, P., Crine, M., Homme, E. L., 1994. The use of large scale computer assited tomography for the study of hydrodynamics in trickling filters, Chem. Eng. Sci. 49, 5271-5280.

$\underline{\mathbf{V}}$

Vlaev, D., Wang, M., Dyakowski, T., Mann, R., Grieve, B.D., 2000. Detecting filter-cake pathologies in solid-liquid filtration: semi-tech scale demonstrations using electrical resistance tomography (ERT). Chem. Eng. J. 77, 87-91.

\section{$\underline{\mathbf{W}}$}

Wang, M., Dorward, A., Vlaev, D., Mann, R., 2000. Measurment of gas-liquid mixing in a stirred vessel using electrical resistance tomography (ERT). Chem. Eng. SCi. 77, 93-98.

Wang, M., Jia, X., Bennet, M., Williams, R.A., 2001. Flow regime identification and optimum interfacial area control of bubble columns using electrical impedance imaging. Proc. $2^{\text {nd }}$ World Cong. On Ind.Process Tomography, Hannover Germany, 726-734.

Wang, M. 2002. Inverse solutions for electrical impedance tomography based on conjugate gradients methods. Meas. Sci. Technol., 13, 101 -117.

Williams, R.A., Jia, X., west, R.M., Wang, M., Cullivan, J.C., Bond, J., Faulks, I., Dyakowski, T., Wang, S.J., Climpson, N., Kostuch, J.A., Payton, D., 1999. Industrial monitoring of hydrocyclone operation using electrical resistance tomography. Minerals. Eng. 12, 1245-1252.

\section{$\underline{\mathbf{Z}}$}

Zlokarnik, M., 2001. Stirring: Theory and Practice. Wiley-VCH: Germany, Weinhim. 